\title{
THE VASCULAR FLORA OF MT SVILAJA (OUTER DINARIDES, SOUTH CROATIA)
}

\author{
Milenko Milović ${ }^{1 *}$, Marija Pandža ${ }^{2}$, Nenad Jasprica ${ }^{3}$, \\ Damira Tafra ${ }^{4} \&$ Vesna Krpina ${ }^{5}$ \\ ${ }^{1}$ Antun Vrančić Grammar School, Šibenik, Croatia, HR-22000 Šibenik, Croatia \\ ${ }^{2}$ Murterski Škoji Primary School, HR-22243 Murter, Croatia \\ ${ }^{3}$ University of Dubrovnik, Institute for Marine and Coastal Research, HR-20000 Dubrovnik, Croatia \\ ${ }^{4}$ Ravnice 20, HR-21310 Omiš, Croatia \\ ${ }^{5}$ Branimira Gušića 3, HR-23000 Zadar, Croatia
}

Milović, M., Pandža, M., Jasprica, N., Tafra, D. \& Krpina, V.: The vascular flora of Mt Svilaja (Outer Dinarides, South Croatia). Nat. Croat., Vol. 30, No. 1, 85-144, 2021, Zagreb.

Based on our own research carried out from 2013 to 2019 and literature data, this study presents for the first time a comprehensive floristic list of Mt Svilaja (max. $1508 \mathrm{~m}$ a.s.l.). It contains 1285 taxa classified within 502 genera and 109 families. Of these, 552 taxa are listed for the first time for Svilaja. Out of 733 taxa recorded previously, the presence of 128 taxa has not been confirmed. Poaceae $(8.79 \%)$, Asteraceae and Fabaceae ( $8.72 \%$ each) had the highest number of taxa, with the largest number of taxa within the genera Trifolium (23 taxa), Carex (20) and Centaurea (17). Hemicryptophytes (43.35\%) and therophytes (25.45\%) prevailed, and Mediterranean (23.27\%), southern European (20.83\%) and Eurasian $(17.43 \%)$ plant taxa dominated. Altogether, there were 76 endemics $(5.91 \%)$, mostly of Illyrian-Adriatic origin, 34 endangered (CR, EN, VU) taxa (2.65\%), and 155 strictly protected taxa according to Croatian law (12.06\%). Among the endemics, the presence of Scabiosa delminiana Abadžić must be stressed. Mt Svilaja is characterized by rich orchid flora (51 taxa, 3.97\%), and features Epipactis placentina Bongiorni \& Grünanger, the only site found in Croatia that does. Although as many as 34 invasive taxa have been recorded, their presence is mostly limited to anthropogenic habitats.

Keywords: Adriatic-Dinaric carbonate platform, biodiversity, chorology, conservation, Dinaric Karst, floristic investigation

Milović, M., Pandža, M., Jasprica, N., Tafra, D. \& Krpina, V.: Vaskularna flora planine Svilaje (vanjski Dinaridi, južna Hrvatska). Nat. Croat., Vol. 30, No. 1, 85-144, 2021, Zagreb.

Na temelju vlastitih istraživanja (2013.-2019.) i literaturnih podataka, u radu je po prvi put prikazan cjeloviti popis vaskularne flore planine Svilaje (1508 m n.v.). Utvrđeno je 1285 svojti, svrstanih u 502 roda i 109 porodica. Od toga su 552 svojte nove za Svilaju, a od 733 svojte prethodno zabilježene u literaturi za njih 128 nalazi nisu potvrđeni. Porodice s najvećim brojem svojti su Poaceae $(8,79 \%)$ te Asteraceae i Fabaceae (obje po 8,72\%), a svojtama najbogatiji rodovi su Trifolium (23), Carex (20) i Centaurea (17). Među životnim oblicima prevladavaju hemikriptofiti $(43,35 \%)$ i terofiti $(25,45 \%)$, a među geoelementima najveći je udio mediteranskih $(23,27 \%)$, južnoeuropskih $(20,83 \%)$ i euroazijskih biljaka $(17,43 \%)$. Udio biljaka od posebnog značaja bio je sljedeći: 76 je endema $(5,91 \%)$, najviše iz skupine Ilirsko-jadranskih, 34 ugroženih (CR, EN, VU; 2,65\%) i 155 strogo zaštićenih (12,06\%) svojti u Hrvatskoj. Među endemima, poglavito ističemo prisutnost vrste Scabiosa delminiana Abadžić, što je drugi nalaz u Hrvatskoj. Svilaja je iznimno bogata orhidejama (51 svojta, 3,97\%) među kojima je i Epipactis placentina Bongiorni \& Grünanger čije je nalazište na Svilaji jedino u Hrvatskoj. Ukupno su tu 34 invazivne svojte, a njihovo rasprostranjenje ograničeno je na antropogena staništa.

Ključne riječi: biološka raznolikost, dinarski krš, floristička istraživanja, Jadransko-dinarska karbonatna platforma, korologija, zaštita i očuvanje

\footnotetext{
*Corresponding author's e-mail: milenko.milovic@si.t-com.hr
} 


\section{INTRODUCTION}

Svilaja (1508 $\mathrm{m}$ a.s.l.) is a mountain located in the central part of the Outer Dinarides in the basement of the Adriatic-Dinaric carbonate platform. Together with the mountains Promina (1147 m), Veliki Kozjak (1206 m), and Moseć (838 m), Mt Svilaja belongs to the central group of Dalmatian mountains located between the higher mountain range of Dinara (1810 m a.s.l.), Troglav (1912 m), and Kamešnica (1855 m), located alongside the borderline of Bosnia and Herzegovina on the one side, and the Adriatic coastal mountains Kozjak (779 m), Mosor (1339 m), and Biokovo (1762 m) on the other (Fig. 1). Due to the karstic relief, the influence of the Mediterranean climate and the high altitude range, the Dalmatian mountains are characterized by a specific flora and vegetation, which is why they have previously been the subject of botanical studies. The floras of the mountains of Biokovo (KuŠan, 1969; RAdić, 1976; ŠILIĆ \& Šolić, 2002), Mosor (Vladović, 1994), Kozjak (Kamenjarin, 1996), and Matokit (Vitasović-Kosić et al., 2020) are well known, while other Dalmatian mountains and hills (Promina, Veliki Kozjak, Svilaja, Moseć, Omiška Dinara, etc.) have not yet been investigated in detail (Milović et al., 2020).

The first data on the flora of Mt Svilaja can be found in classical works from the $19^{\text {th }}$ century (PetTer, 1832; VISIANI 1842-1852, 1872). At the beginning of the $20^{\text {th }}$ century, the Austrian botanist JANCHEN $(1908,1910)$ reported about 30 plant species from Svilaja. Since the 1960s, however, botanists have only occasionally and incidentally visited Svilaja and published partial floristic records (Jedlovski, 1952; MARKović,

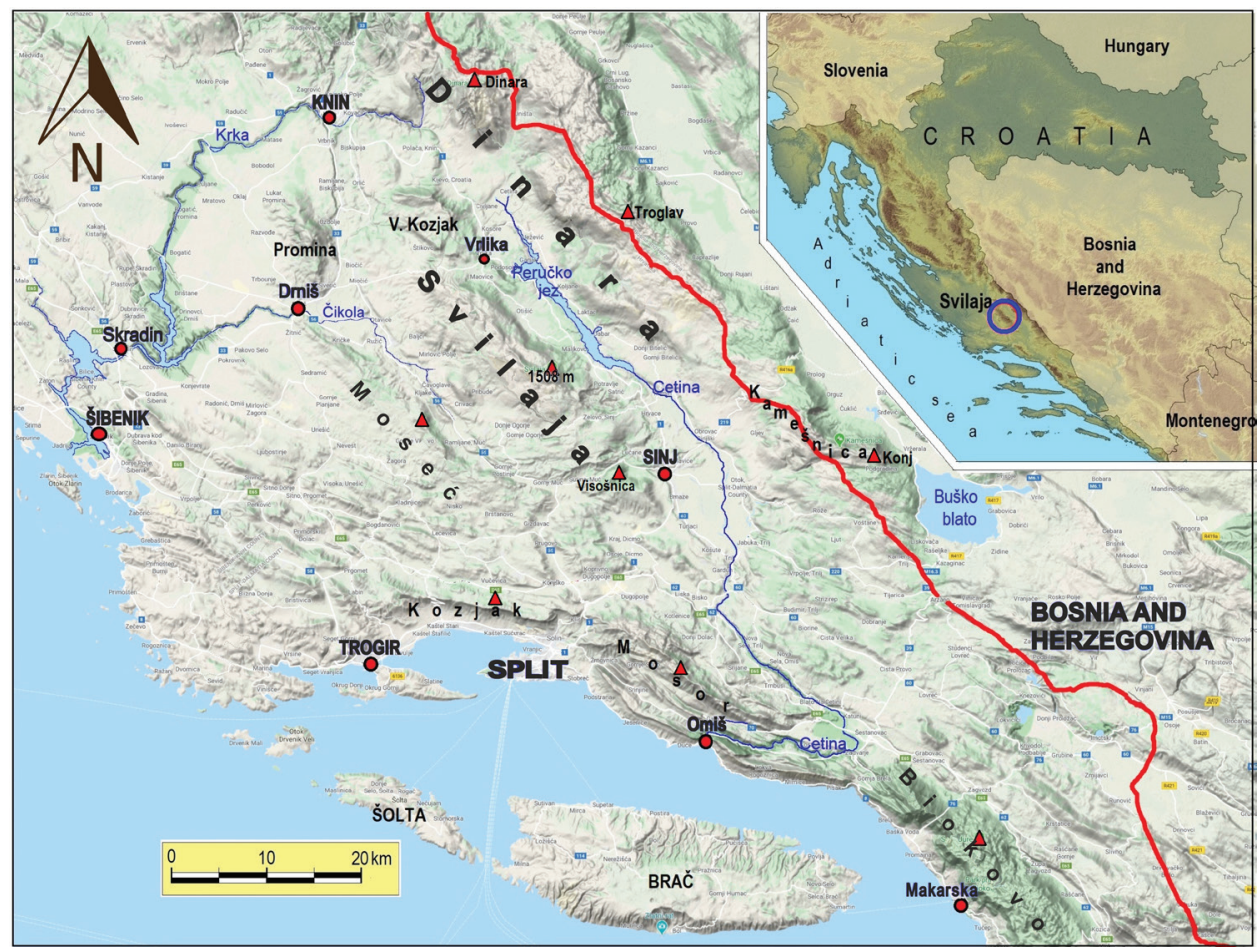

Fig. 1. Geographical position of the Mt Svilaja in the Dalmatia hinterland (Dalmatinska Zagora), South Croatia. 
1964, 1992; Marković-Gospodarić, 1965; Lovrić et al., 1987, 1989; Lovrić \& RAC, 1989; Trinajstić, 1992; Kranjčev, 2005; Delforge, 2006a; Milović et al., 2013; Milović \&


al., 2012, 2019). A historical overview of research into the flora and vegetation of $\mathrm{Mt}$ Svilaja is presented in detail by Milović et al. (2020).

Strong depopulation, frequent wildfires, reduction of the intensity of traditional animal husbandry and agriculture have significantly affected the current state of flora and vegetation (DURBEŠIĆ \& FUERST-BJELIŠ, 2016). These changes when combined with historical data indicate the need for comprehensive research into the flora of Svilaja.

The aim of our study research is to finalise an updated comprehensive inventory of vascular flora on Mt Svilaja by tracking current and past records. The present study also includes floristic analysis, and takes into consideration life form spectrum and types of geolements, the contribution of endemic, endangered, protected and invasive taxa. The results of the research will be an important contribution to the knowledge of the recent state of the vascular plant flora of Mt Svilaja and, at the same time, the chorology of plant species in Croatia.

\section{STUDY AREA}

Svilaja is a mountain in the Dalmatia hinterland (Dalmattinska Zagora) located between the towns of Vrlika and Sinj, and extends from northwest to southeast. On the west and south it is separated from the surrounding mountains and hills by karstic fields (Vrličko Polje, Sinjsko Polje, Mućko Polje and Petrovo Polje) with the altitude varying from 300 to $400 \mathrm{~m}$ a.s.l. It is bordered by the upper course of the Čikola River and the torrent streams Vrba, Sutina and Karakašica on the west and south, and by the Cetina River with Lake Peruča on the east.

The highest peak of Mt Svilaja (Svilaja Bat or Svilaja, $1508 \mathrm{~m}$ a.s.l.) rises from the north of Sinj, approximately $30 \mathrm{~km}$ from the Adriatic coast. In the northwestern part (between Vrlika and Knin) Svilaja crosses into the ridge of Veliki Kozjak, separated by a road between the towns of Drniš - Vrlika and Lemeš Pass ( $860 \mathrm{~m}$ a.s.l.). The road between the villages of Muć and Lučane separates the southern slopes of Svilaja from Visošnica (941 m a.s.l.). The length of Svilaja (not including Veliki Kozjak and Visošnica) is approximately $30 \mathrm{~km}$, and the average width is $15 \mathrm{~km}$. The southwestern slopes descend in a slightly terraced manner towards Petrovo Polje, while the southeastern slopes from the highest ridges (Svilaja - Jančag) fall steeply towards the Cetina River and Lake Peruča.

The geological bedrock consists of carbonate rocks, the most common being limestones and dolomites of predominantly Jurassic and Triassic age (BOJANIĆ, 1961; PAPEŠ et al., 1982). In the vicinity of the village of Maovice there are more dilapidated limestone breccias, and the dolomites are more present on the southern slopes of Svilaja towards Muć and Sinj. Silicate terrains in the form of metamorphic rocks (Werfen shales) also occur on Svilaja, for example in the vicinity of Muć, while eruptive rocks have been identified in the vicinity of Vrlika and Sinj (BARIĆ, 1957).

Among the soils, brown soils on limestone (Calcocambisol) predominate on Svilaja, while black soil on limestone (Calcomelanosol), shallow and medium-deep red soils, rendzina on dolomite and dolomitized limestone are much less represented (Vukadinović, 2019). These soils are unsuitable or limitedly suitable for growing plants due to their small depth, excessive water permeability, poor sorption ability 
(due to textural, structural and chemical properties) and susceptibility to erosion (Bogunović et al., 2006). Soils are exposed to strong erosion, especially on the southern slopes of Svilaja (the villages of the Ogorje and Muć) where efforts are made to mitigate this process by regulating watercourses and afforestation with black pine (Durbešić \& Milković, 2005; Čović 2017).

The relief of the wider area of Mt Svilaja is characterized by the specific underground water circulation, while most of the surface flows are temporary and torrential, and include the upper course of the Čikola River with its tributaries Vrba, Sutina, Karakašica and Vrlika. Therefore, rare springs, wells, temporary and permanent ponds are of great importance for the human population and livestock on the $\mathrm{Mt}$ Svilaja Surface run-off water quickly infiltrates the soil and flows through the underground network of watercourses. The Mt Svilaja range is the watershed of two river basins - of the Krka and Cetina rivers (BRKIĆ et al., 2006).

According to Köppen's climate classification (Köppen \& Geiger, 1954; Sträßser, 1998), the wider area of Mt Svilaja has a moderately warm humid climate with hot summers or a Cfsa type of climate, and in the area of peak ridges a Cfsb type of climate, moderately warm and humid with warm summers (Š́EGOTA \& FILIPČIĆ, 2003: 35). The mean annual air temperature is $13^{\circ} \mathrm{C}$, the coldest month is January $\left(3.8^{\circ} \mathrm{C}\right)$, and the warmest is July $\left(23.4^{\circ} \mathrm{C}\right.$; data from the meteorological station in Sinj for the period 1989-2018, Croatian Meteorological and Hydrological Service). The mean annual precipitation is $1147 \mathrm{~mm}$, the lowest in July $(45.8 \mathrm{~mm})$ and the highest in November (170.2 mm).

Phytogeographycally, Mt Svilaja belongs to the Illyrian sector of the Apennine-Balkan province of the Euro-Siberian region (Rivas-MarTínez et al., 1982, 2004). Deciduous vegetation is present in the lower altitudes and belongs to the thermophilic deciduous oak forests of the vegetation class Quercetea pubescentis Doing-Kraft ex Scamoni et Passarge 1959, while in the higher belts mesophilic deciduous and mixed forests of the class Carpino-Fagetea sylvaticae Jakucs ex Passarge 1968 (Mucina et al., 2016; ŠKVORC et al., 2017) occur. Unlike some of the coastal Dalmatian mountains (Kozjak, Mosor and Biokovo), due to the long distance from the Adriatic Sea, Mt Svilaja doesn't include a belt of Mediterranean evergreen holm oak forests (KuŠAN, 1969: 104).

Along with natural factors, human activity has had a long-lasting and significant influence on the current state and features of the vegetation on Svilaja. In the $19^{\text {th }}$ and in the first half of the $20^{\text {th }}$ century, the area under forests was significantly reduced (i.e., cleared) to provide space for pastures and arable land (DURBEŠIĆ \& FuERST-BJELIš, 2016). In the last 50 years there has been a reverse trend due to the effects of emigration and the related reduction of areas used for grazing. In some areas there are abandoned arable fields and pastures overgrown with thickets and forests. Surface areas with thickets and forests would have been significantly larger if there had not been frequent wildfires during the last 20 years the extinguishing of which was difficult or impossible due to the inaccessible terrain and mines left after the Homeland War (Durbešić \& Fuerst-BJELIŠ, 2016). More recently, the construction of wind turbines on Svilaja has been intensified, which further disturbs the natural landscape and devastates the vegetation. Wind turbines have been built in the area of the village of Ogorje (Kokani), and new ones are planned to be built near the village of Zelovo and on the hills of Visošnica and Visoka, etc. (MEsarić, 2016).

A more detailed overview of the natural features of Mt Svilaja is presented in the paper given by Milović et al. (2020). 


\section{MATERIALS AND METHODS}

This study covered the investigated area on Mt Svilaja within the boundaries shown in Fig. 2. The northern border was the road between Drniš and Vrlika, from the settlements Miočić to Vrlika, including the settlements Maovice and Vrlika. The eastern border was the road from Vrlika - Sinj to Karakašica. The south-western border lies on the road between Sinj and Drniš, passing from Karakašica through the villages of Muć, Ramljane, Kljake, Ružić, Gradac and again to Miočić. The altitudes of the investigated area range from 280 to $1508 \mathrm{~m}$ a.s.l. The total surface area of the study area is ca $418 \mathrm{~km}^{2}$.

Floristic research was conducted in the period from 2013 to 2019 using the standard method (Nikolić et al., 1998; Nikolić, 2006). All seasons and different types of natural and anthropogenic habitats were covered. Altogether, 70 localities were investigated within the boundaries described above (Fig. 2). For each locality, the Gauss-Krüger coordinates of the central point were taken, and vascular plant taxa in a radius of approximately 100-200 m were recorded. Some localities were visited several times from February to October, most of them at least twice a year, but a few only once.

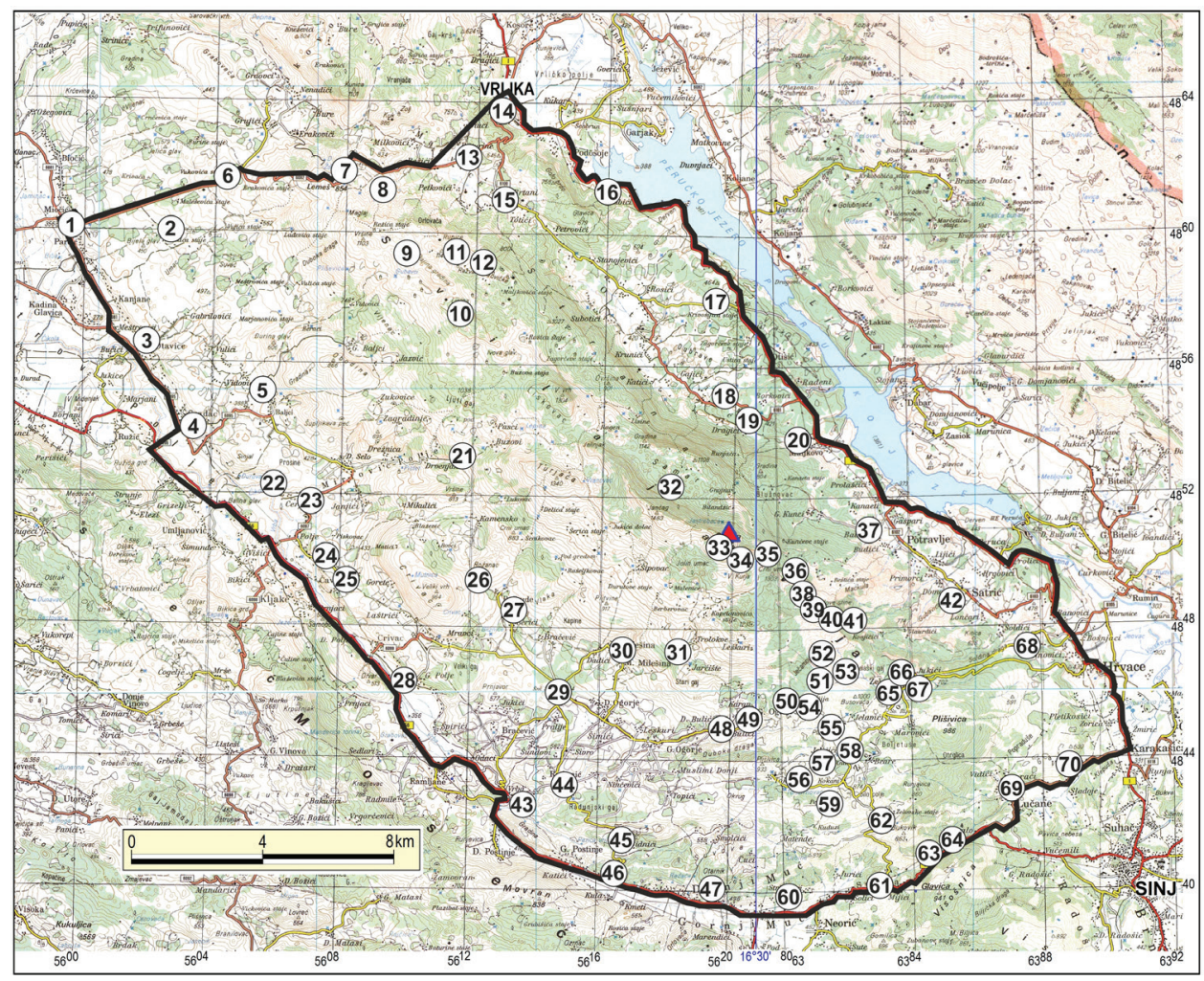

Fig. 2. The borders of the studied area on the Mt Svilaja with the position of the investigated localities (1-70, see Tab. 1). 
The list of taxa includes all species and subspecies found to grow wild in the area. Of the cultivated taxa, only those that have the ability to spread outside cultivation ("gardenscapes") were recorded. Previously recorded taxa not confirmed by this study are also included. Plant taxa found exclusively in cultivation are shown in a separate list (App. 2, Tab. 2), and were not included in the analysis of flora.

Taxa were identified using the standard keys, books and guides: TuTin et al. (19681980, 1993), Pignatti (1982), Domac (1994), Nikolić (2019), Walters et al. (1986-2000), Delforge (2006). The nomenclature of plant taxa, including higher taxonomic rank, mainly follows Flora Croatica Database, FCD, (Nikolić, 2020a). For a number of taxa not represented in FCD, Walters et al. (1986-2000) and Delforge (2006) were used, and these were marked with an asterisk $\left.{ }^{*}\right)$ in the floristic list.

Biological form was verified in the field and denoted according to categories reported in Pignatti (1982), these being based on the classification of RAUnKiaER (1934): Ch (Chamaephyta), G (Geophyta), H (Hemicryptophyta), Hy (Hydrophyta), P (Phanerophyta) and T (Therophyta).

Regarding chorological form, the classification of the plant taxa into floral elements and lower categories has been performed according to HoRvaTić et al. (1967/1968). The names of the floral elements with the corresponding abbreviations used in the floristic list are shown in Tab. 7 in the Results and Discussion section.

Plant taxa considered endemic to Croatia (Nikolić et al., 2015) are marked with the abbreviation "end". Croatian statutorily strictly protected taxa, defined by Croatian laws (AnOnymous, 2013), are marked as "sp". The threat status of taxa was determined according to the Red Book of Vascular Flora of the Republic of Croatia (NikOLIĆ \& TopIć, 2005; Nikolić, 2020b) and marked using the abbreviations as follows: CR (Critically Endangered), EN (Endangered), VU (Vulnerable). According to precautionary criteria, the following categories were also highlighted: NT (Near Threatened), DD (Data Deficient), and LC (Least Concern). The taxa that are considered invasive alien species in Croatia (Boršıć et al., 2008; Nikolić et al., 2014; Nikolić, 2020c) are accompanied by the abbreviation "inv".

Locality numbers (1-70, see Fig. 2 and Tab. 1) were attributed to each taxon. In addition, for taxa reported from the literature, the author name and corresponding year were indicated. Should previous records not have been confirmed during this study, only the name of locality (toponym) has been used, as presented in the original publication. In the list of flora (App. 1, Tab. 1), the author name abbreviations were used as follows:

A-1911 (Adamović, 1911); A-1929 (Adamović, 1929); De-2010 (Delić, 2010); Df-2006 (Delforge, 2006), Ja-1908 (Janchen, 1908); Ja-1910 (Janchen, 1910); Je-1952 (Jedlovski, 1952); Kr-2005 (KranjčEv, 2005); K\&S-2016 (KranjČEv \& ŠEŠOK, 2016); Ku-1969 (KuŠAN, 1969); L\&al-1987 (Lovrić et al., 1987); L\&R-1989 (Lovrić \& RAC, 1989); LRS-1989 (Lovrić et al., 1989); Ma-1964 (Marković, 1964); Ma-1992 (Marković, 1992); Me-1972 (MeŠTrović, 1972); VRZ-2012 (Vladović et al., 2012); V\&al-2019 (Vladović et al., 2019); Mi\&al-2013 (Milović et al., 2013); M\&K-2016 (Milović \& KaraĐole, 2016); Pe-1832 (Petter, 1832); Se-2016 (ŠEGOTA, 2016); Se\&al-2016 (Š́EGOTA et al., 2016); Tr-1992 (TRINAJSTIĆ, 1992), Vi-1842 (VISIANI, 1842); Vi-1847 (VISIANI, 1847); Vi-1852 (VISIANI, 1852); Vi-1872 (VISIANI, 1872). 
Tab. 1. List of the investigated localities with their names and position (Gauss-Krüger's coordinates, $5^{\text {th }}$ and $6^{\text {th }}$ zone, see also Fig. 2).

\begin{tabular}{|c|c|c|c|}
\hline \multirow{2}{*}{$\begin{array}{l}\text { No. of } \\
\text { locality }\end{array}$} & \multicolumn{2}{|c|}{$\begin{array}{l}\text { Gauss-Krüger's } \\
\text { coordinates }\end{array}$} & \multirow{2}{*}{ Locality } \\
\hline & $x$ & Y & \\
\hline 1 & 4859985 & 5600056 & Miočić \\
\hline 2 & 4859944 & 5602552 & Raketići stables \\
\hline 3 & 4856540 & 5601988 & Otavice \\
\hline 4 & 4853861 & 5603512 & Gradac, near the church and cemetery \\
\hline 5 & 4855286 & 5605421 & Gradac, Vidovići \\
\hline 6 & 4861624 & 5604635 & Miočić-Vrlika road, surroundings of the turn for Štikovo \\
\hline 7 & 4861952 & 5607956 & The surroundings of Lemeš Pass \\
\hline 8 & 4861423 & 5607038 & Lemeš Pass \\
\hline 9 & 4859367 & 5609718 & Surroundings of Režići stables \\
\hline 10 & 4857749 & 5611484 & Peak of Lisina and surroundings \\
\hline 11 & 4859386 & 5611243 & Mountain house on Sv. Jure \\
\hline 12 & 4859190 & 5612017 & Razvale Maovičke \\
\hline 13 & 4862180 & 5611422 & Maovice, between Režići and Baturine \\
\hline 14 & 4863824 & 5612530 & Vrlika \\
\hline 15 & 4861217 & 5612960 & Maovice, the surroundings of Totići and Brtani \\
\hline 16 & 4861460 & 6375199 & Grabići \\
\hline 17 & 4857913 & 5619199 & Otišić \\
\hline 18 & 4855040 & 5619785 & Otišić, between Gajići and Dragići \\
\hline 19 & 4854512 & 5620313 & Otišić, the surroundings of Dragići \\
\hline 20 & 4853659 & 6380658 & Maljkovo \\
\hline 21 & 4853082 & 5610997 & Mirlović Polje, Drvenjak \\
\hline 22 & 4852198 & 5605841 & Mirlović Polje, the confluence of Vrba and Čikola \\
\hline 23 & 4851882 & 5607219 & Mirlović Polje, Cerje \\
\hline 24 & 4849815 & 5607759 & Between Polje and Čavoglave \\
\hline 25 & 4849308 & 5608451 & Čavoglave \\
\hline 26 & 4849243 & 5612163 & Northwest above Pribude \\
\hline 27 & 4848291 & 5613089 & Pribude, Šerići \\
\hline 28 & 4846194 & 5609929 & Gornje Polje, Crivac \\
\hline 29 & 4846196 & 5614805 & Donje Ogorje, the surroundings of the All Saints' Church \\
\hline 30 & 4847221 & 5616514 & Velika Milešina and surroundings \\
\hline 31 & 4847265 & 5618393 & Milešina, Golubinka cave \\
\hline 32 & 4852270 & 5617796 & Samar \\
\hline 33 & 4850656 & 5619878 & Svilaja Peak \\
\hline 34 & 4850298 & 5620056 & The surroundings of Svilaja Peak \\
\hline 35 & 4850066 & 6379870 & The northern slope of Crni Umac \\
\hline 36 & 4849748 & 6380271 & Hiking trail east of Crni Umac \\
\hline 37 & 4850962 & 6382841 & Potravlje, Babići \\
\hline 38 & 4848653 & 6380774 & Hiking trail between Crni Umac and Orlove Stine \\
\hline 39 & 4848406 & 6381207 & The surroundings of the hiking lodge Orlove Stine \\
\hline 40 & 4848349 & 6381386 & The surroundings of the hiking lodge Orlove Stine \\
\hline 41 & 4848174 & 6381626 & The surroundings of the hiking lodge Orlove Stine \\
\hline 42 & 4848724 & 6385433 & Satrić, between Cvitkovići and Domazeti \\
\hline 43 & 4843221 & 5613153 & Vrba \\
\hline 44 & 4843194 & 5615174 & Radunić \\
\hline 45 & 4841573 & 5616642 & Bidnići \\
\hline 46 & 4840382 & 5616495 & Muć, along the Suvova stream \\
\hline 47 & 4840149 & 5618757 & Muć, Šegovića Brig \\
\hline 48 & 4844922 & 5619850 & Ogorje, between Bulići and Čolaci \\
\hline 49 & 4845122 & 6378960 & Gornje Ogorje, Gornji Bulići \\
\hline
\end{tabular}




\begin{tabular}{|c|c|c|c|}
\hline \multirow{2}{*}{$\begin{array}{l}\text { No. of } \\
\text { locality }\end{array}$} & \multicolumn{2}{|c|}{$\begin{array}{l}\text { Gauss-Krüger's } \\
\text { coordinates }\end{array}$} & \multirow{2}{*}{ Locality } \\
\hline & $x$ & $\mathrm{Y}$ & \\
\hline 50 & 4845708 & 6380392 & Gornje Ogorje, Gornji Muslimi \\
\hline 51 & 4846414 & 6381227 & Gornje Ogorje, northeast above Tešije \\
\hline 52 & 4847008 & 6381255 & Hiking trail between Jačmenjaci and Gole Kose \\
\hline 53 & 4846437 & 6381812 & Zelovo-G. Ogorje road, along the N-slopes of Busovača hill \\
\hline 54 & 4845633 & 6380774 & Gornje Ogorje, between Tešije and Gornji Muslimi \\
\hline 55 & 4844812 & 6381290 & Gornje Ogorje, between Tešije and Jeličići \\
\hline 56 & 4843247 & 6380664 & Zelovo, between Tokići and Kokani \\
\hline 57 & 4843950 & 6381117 & Zelovo, the surroundings of St Anne's Church \\
\hline 58 & 4844228 & 6381825 & Zelovo, the surroundings of Jeličići \\
\hline 59 & 4842708 & 6381604 & Gornje Ogorje, the surroundings of Kokani \\
\hline 60 & 4839861 & 5621829 & Muć, the surroundings of Stričevići \\
\hline 61 & 4840061 & 6382881 & Sutina, the surroundings of Mijići \\
\hline 62 & 4842058 & 6382962 & Zelovo stables, along the road Zelovo-Sutina \\
\hline 63 & 4841226 & 6384854 & Sutina stream, beech forest, the west of Đipalo \\
\hline 64 & 4841510 & 6385376 & Sutina stream, the west of Đipalo \\
\hline 65 & 4846035 & 6383355 & Zelovo, the surroundings of Lađa \\
\hline 66 & 4846347 & 6383669 & Zelovo, Domazeti \\
\hline 67 & 4846264 & 6384095 & Zelovo, the surroundings of Jukići \\
\hline 68 & 4847205 & 6387655 & A quarry, along the Hrvace-Zelovo road \\
\hline 69 & 4843168 & 6387000 & Lučane \\
\hline 70 & 4843947 & 6388970 & Karakašica \\
\hline
\end{tabular}

\section{RESULTS AND DISCUSSION}

\section{Flora}

Based on the literature data and our own research, the first comprehensive list of vascular flora of Mt Svilaja was made (App. 1, Tab. 1). Altogether, it contains 1285 taxa i.e., nearly $25 \%$ of the total flora in Croatia (Nikolić, 2020a). In addition, 56 taxa were found exclusively in cultivation (App. 2, Tab. 2). The separate list of cultivated taxa can serve as a starting point for some future research of cultivated flora in the area.

The total number of taxa on Mt Svilaja is higher than on Mosor (872 taxa), Kozjak (604), and Matokit (604), and more or less similar to those on Risnjak (1148 taxa), Papuk (1223) and Biokovo ( 1400) (Tab. 2). These differences can be explained by size and altitude range of the massifs, geographical position, climatic conditions and related phytogeographical characteristics, and degree of the scientific knowledge about each of them. For example, the lower number of taxa on Svilaja than on Biokovo was expected due to the absence of taxa from the Mediterranean-climate evergreen ecosystems [e.g., Arbutus unedo L., Pinus halepensis Mill., Aurinia sinuata (L.) Griseb., taxa of the genus Cistus L., Euphorbia characias L. subsp. wulfenii (Hoppe ex W.D.J.Koch) Radcl.Sm. (found only in cultivation), Juniperus phoenicea L., Quercus ilex L., Tanacetum cinerariifolium (Trevir.) Sch.Bip., etc.]. In this study, Salvia officinalis L. was very rare and found with a few individuals only near the village of Lemeš ( $\mathrm{x}=$ 4861547, y = 5607414). Vladović et al. (2019) have reported it for Sutina. In addition, LOVRIĆ et al. (1987) observed that the association Stipo-Salvietum officinalis Horvatić (1956) 1958 is frequent on the lower altitudes of Svilaja. 
Tab. 2. The number of taxa (species and infraspecific taxa) on the Mt Svilaja and its comparison to other mountains in Croatia.

\begin{tabular}{|l|c|c|c|c|}
\hline \multicolumn{1}{|c|}{ Mountain } & $\begin{array}{c}\text { Max. altitude } \\
\text { (m a.s.l. })\end{array}$ & $\begin{array}{c}\text { Vegetation } \\
\text { region }\end{array}$ & $\begin{array}{c}\text { No. of } \\
\text { taxa }\end{array}$ & Source \\
\hline Biokovo & 1764 & Mediterranean & $\sim 1400$ & ŠILIĆ \& ŠOLIĆ, 2002 \\
\hline Matokit & 1062 & Mediterranean & 604 & VITASOvić-KosIĆ et al., 2020 \\
\hline Mosor & 1340 & Mediterranean & 872 & VLADOvić, 1994 \\
\hline Kozjak & 779 & Mediterranean & 604 & KAMENJARIN, 1996 \\
\hline Svilaja & 1508 & Euro-Siberian & 1285 & This study \\
\hline Risnjak & 1528 & Euro-Siberian & 1148 & https://np-risnjak.hr/flora/ \\
\hline Papuk & 953 & Euro-Siberian & 1223 & PANDŽA, 2010 \\
\hline
\end{tabular}

The number of taxa recorded should not be considered final. Some localities, particularly at higher altitudes, were impossible to visit in all seasons. Subsequent research should further reconsider some doubtful and dated records of taxa. Although this study was conducted at 70 localities that cover the entire area of Svilaja, it still gives only a partial insight into the distribution of individual taxa.

\section{Previously recorded and doubtful taxa}

Of the total number of taxa (1285) on Mt Svilaja, 1157 taxa were found during our own field research from 2013 to 2019. Among them, 552 taxa are listed for the first time for the mountain. We did not confirm findings for 128 of the 733 previously recorded taxa. Findings of some taxa reported from the literature still remain dubious, and further investigation needs to be done to complete the picture of flora.

In this study, we have omitted several previously recorded orchid taxa (KRANJčEv, 2005). Ophrys corniculata R.Kranjčev is not valid. Additionally, some taxa reported from Delforge (2006a, b) and Nikolić (2020a) do not occur on the territory of Croatia: Ophrys holubyana Andrasovszky, O. philippei Gren., O. aegirtica P.Delforge, O. annae Devillers-Tersch. \& Devillers, O. gracilis (Büel, O.Danesch \& E.Danesch) Englmaier, O. linearis (Moggr.) P. Delforge, Devillers \& Devillers-Tersch. Micromeria parviflora Rchb. and Minuartia juniperina (L.) Maire \& Petit, reported by Lovrić \& RAC (1989) for "Svilaja Mountain range" (Svilaja and Veliki Kozjak), were also omitted, since Flora Croatica Database (FCD) does not recognize them as members of the Croatian flora (Nikolić, 2020a). In addition, the taxon Hieracium balbisianum Arv.-Touv. \& Briq. was also not included in the floristic list. JENCHEN (1908) found it on the southern slopes of Svilaja, but his finding has never been confirmed, including this study. As a consequence it was not included in FCD (Nikolić, 2020a).

Previously, only about 400 taxa were known for the Svilaja area (Mrlović et al., 2020). These data originated from the published data in the $19^{\text {th }}$ and early $20^{\text {th }}$ centuries (Petter, 1832; Visiani, 1842, 1847, 1852, 1872; Janchen, 1908), in the second half of the last century (Marković, 1964, 1992; Lovrić et al., 1987, 1989; Lovrić \& RAC, 1989, TrinajSTIĆ, 1992) and in more recent articles (KRANJČEV, 2005; ŠEGOTA, 2016; ŠEgOTA et al., 2016, Milović et al., 2013, Milović \& KARAĐOLE, 2016). The largest number of previously recorded taxa (519) originated from the recently published professional publication "Significant Landscape of Sutina" (Vladović et al., 2019). However, we did not 
include several taxa from this publication, i.e. those taken from Degen et al. (1908), because Sutina is not only a site on Svilaja, but a site with the same name exists on the slopes of Mt Dinara near the village of Ježević, an area not included in this study. JANCHEN (1908: 77) has already drawn attention to the confused state of records at localities that have the same name.

In our study, among the taxa listed in VLADOvić et al. (2019), as many as 66 taxa were not confirmed. Actually, half of the surface area of "Sutina" is located outside the boundaries of our study area (on the northern slopes of the hill Visošnica).

We have included several endemic taxa from previous studies, although some of them can be considered doubtful for the mountain. Among them, the record of Asperula scutellaris Vis. is doubtful (Lovrić \& RAC 1989, "Svilaja mountain range"). According to Bogdanović (2015a), this plant species occurs only on Vis Archipelago and Dugi Otok island, while other records (e.g., the island of Pag, Mt Svilaja) are also doubtful.

The Croatian endemic Chouardia lakusicii (Šilić) Speta reported by ŠEGOTA (2016) from the southern slopes of Svilaja may also be reconsidered. In this study, this species was not found. As the morphologically very similar Ch. litardierei (Breistr.) Speta is abundant in the surrounding flooded areas and on damp habitats (Cetinsko Polje, Vrličko Polje and Sinjsko polje, the Sutina stream), we assume that the previously noted record of $\mathrm{Ch}$. lakusicii should most likely really be that of $\mathrm{Ch}$. litardierei, whose seeds have been spread by birds to dry grasslands. The first author of this article has also found individuals of $\mathrm{Ch}$. litardierei on a dry meadow along the edge of the Čikola Canyon near the village of Brnjica.

The presence of Crocus tommasinianus Herb. is also doubtful. Lovrić et al. (1989) reported this species from the highest altitude grasslands ("Svilaja Mountain range") of Svilaja and Veliki Kozjak, and ŽEvrnja \& Vladović (2005) for the village of Neorić. The photo of "C. tommasinianus" from ŽEvrnJA \& Vladović (2005) includes the purple tube of the perigone and it may be concluded that the taxon actually belongs to $C$. purpureus Weston [C. neapolitanus (Ker Gawl.) Ascherson], distributed over the entire Svilaja, and also frequent in Neorić.

\section{Floristic analysis}

The vascular flora of Svilaja contains 1285 species and subspecies classified within 502 genera and 109 families (Tab. 3). The average number of taxa per family and genus is 11.79 and 2.56, respectively.

The families with the highest number of taxa (Tab. 4) were Poaceae (113 taxa, $8.79 \%$ ), Asteraceae s. str. and Fabaceae (112 taxa each, 8.72\%), which together made up $26.23 \%$ of the taxa of the total vascular flora on Svilaja. These families also prevail in the flora of some other areas in Croatia: Šibenik and its surroundings (MILović, 2002), Papuk (PandžA, 2010), Biokovo (Radić, 1976), Mosor (Vladović, 1994) and Matokit (Vitasović-Kosić et al., 2020), as well as in the total flora of Croatia (Nikolić \& Topić, 2005).

Sixteen genera contained more than 10 taxa. The genera with the highest number of taxa were: Trifolium (23 taxa), Carex (20), and Centaurea (17) (Tab. 5). These taxa contributed the most in the vegetation of dry grasslands on Svilaja. 
Tab. 3. The number of taxa of the flora of Mt Svilaja

\begin{tabular}{|l|c|c|c|c|c|}
\hline \multirow{2}{*}{ Taxon } & \multirow{2}{*}{ Pteridophytes } & \multirow{2}{*}{ Gymnosperms } & \multicolumn{2}{c|}{ Angiosperms } & \multirow{2}{*}{ Total } \\
\cline { 4 - 6 } & & & Dicots & Monocots & \\
\hline Families & 7 & 2 & 84 & 16 & 109 \\
\hline Genera & 8 & 3 & 390 & 101 & 502 \\
\hline Species & 17 & 4 & 817 & 236 & 1074 \\
\hline Subspecies & 2 & 4 & 167 & 38 & 211 \\
\hline Species + subspecies & 19 & 8 & 76.58 & 21.32 & 1285 \\
\hline \% of total no. of taxa & 1.48 & 0.62 & & & 100 \\
\hline
\end{tabular}

Tab. 4. List of families including more than 20 taxa.

\begin{tabular}{|l|c|c|}
\hline \multicolumn{1}{|c|}{ Family } & No. of taxa & $\begin{array}{c}\text { \% of total } \\
\text { no. of taxa }\end{array}$ \\
\hline Poaceae & 113 & 8.79 \\
\hline Asteraceae & 112 & 8.72 \\
\hline Fabaceae & 112 & 8.72 \\
\hline Lamiaceae & 61 & 4.75 \\
\hline Cichoriaceae & 58 & 4.51 \\
\hline Apiaceae & 55 & 4.28 \\
\hline Scrophulariaceae & 55 & 4.28 \\
\hline Brassicaceae & 53 & 4.12 \\
\hline Caryophyllaceae & 53 & 4.12 \\
\hline Orchidaceae & 51 & 3.97 \\
\hline Rosaceae & 49 & 3.81 \\
\hline Ranunculaceae & 30 & 2.33 \\
\hline Cyperaceae & 26 & 2.02 \\
\hline Asparagaceae & 24 & 1.87 \\
\hline Boraginaceae & 21 & 1.63 \\
\hline Other families (94) & 412 & 32.06 \\
\hline Total (109) & 1285 & 100.00 \\
\hline
\end{tabular}

Tab. 5. List of genera containing more than 10 taxa.

\begin{tabular}{|l|c|}
\hline \multicolumn{1}{|c|}{ Genus } & No. of taxa \\
\hline Trifolium & 23 \\
\hline Carex & 20 \\
\hline Centaurea & 17 \\
\hline Ophrys & 14 \\
\hline Ranunculus & 14 \\
\hline Allium & 13 \\
\hline Vicia & 13 \\
\hline Bromus & 12 \\
\hline Crepis & 12 \\
\hline Lathyrus & 12 \\
\hline Orchis & 12 \\
\hline Silene & 12 \\
\hline Veronica & 12 \\
\hline Festuca & 11 \\
\hline Geranium & 11 \\
\hline Verbascum & 11 \\
\hline
\end{tabular}

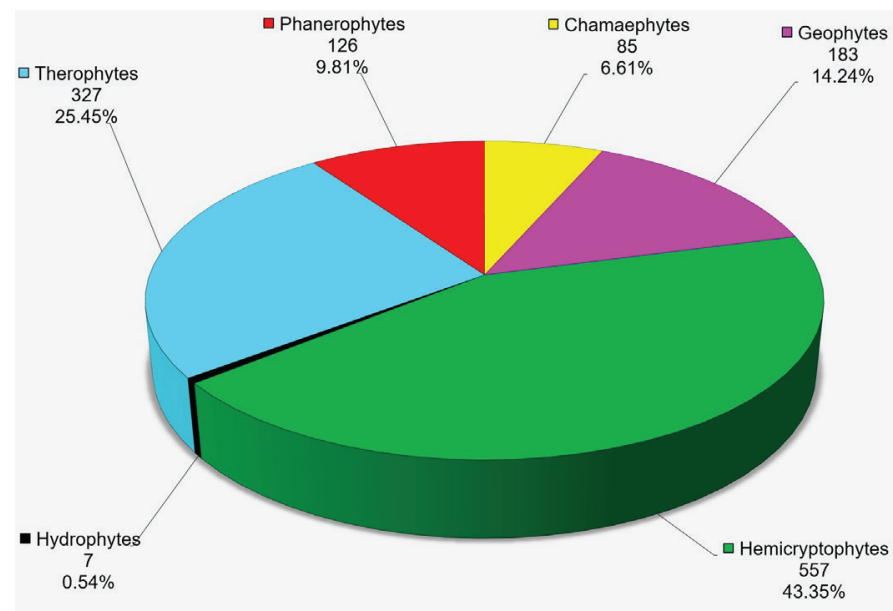

Fig. 3. Life-form spectrum on the Mt Svilaja. 


\section{Analysis of life forms and geoelements}

The analysis of life forms (Fig. 3) showed the predominance of hemicryptophytes (557 taxa, 43.35\%) and therophytes (327, 25.45\%). Geophytes (14.24\%) and phanerophytes $(9.81 \%)$ contributed significantly lower. The dominance of hemicryptophytes and therophytes is in accordance with the climatic conditions and the dominant types of vegetation units (RAunkier, 1934; Horvat, 1949, Milović et al., 2020).

The mountain is located on the border of the Euro-Siberian and Mediterranean vegetation regions, where the climate becomes colder and wetter as the altitude increases, and the influence of the Mediterranean climate becomes weaker. The vegetation is dominated by grasslands, thickets and forests which often contain hemicryptophytes. Therophytes frequently occur in the anthropogenic habitats (settlements, agricultural areas) located on the lower slopes of Svilaja. However, for a simple illustration, Svilaja had a lower percentage contribution of hemicryptophytes and a higher of therophytes than Papuk (in continental Croatia) and vice versa. Additionally, a higher contribution of hemicryptophytes and a lower of therophytes were found on Mosor due to lower altitudes and closer proximity to the Adriatic coast, and Šibenik (coastal area with a Mediterranean climate) as well as in the spectrum of life forms of the Mediterranean as a whole (Tab. 6).

Tab. 6. Life-form spectrum of Mt Svilaja in comparison to Mt Mosor (Vladović, 1994), Mt Papuk (PAndžA, 2010), Mt Matokit (Vitasović-Kosić et al., 2020), Šibenik and surroundings (Milović, 2002) and in the Mediterranean region as a whole (Horvat, 1949). P - phanerophytes, Ch - chamaephytes, H - hemicryptophytes, G - geophytes, T - therophytes, Hy - hydrophytes.

\begin{tabular}{|c|c|c|c|c|c|c|}
\hline \multirow{2}{*}{ Area } & \multicolumn{7}{|c|}{ Life-form (\%) } \\
\cline { 2 - 7 } & $\mathrm{P}$ & $\mathrm{Ch}$ & $\mathrm{H}$ & $\mathrm{G}$ & $\mathrm{T}$ & Hy \\
\hline Mediterranean & 12 & 6 & 29 & 11 & 42 & - \\
\hline $\begin{array}{c}\text { Šbenik and } \\
\text { surroundings }\end{array}$ & 8.84 & 6.51 & 30.33 & 10.51 & 42.79 & 1.02 \\
\hline Mt Mosor & 14.92 & 9.28 & 35.14 & 11.79 & 28.43 & 0.47 \\
\hline Mt Svilaja & 9.81 & 6.61 & 43.35 & 14.24 & 25.45 & 0.54 \\
\hline Mt Matokit & 10.76 & 10.76 & 39.90 & 12.42 & 26.16 & - \\
\hline Mt Papuk & 12.43 & 3.52 & 48.16 & 12.92 & 20.52 & 2.45 \\
\hline
\end{tabular}

The Mediterranean geoelement (299 taxa, 23.27\%), mostly circum-Mediterranean plants, was followed by a considerable proportion of South European $(269,20.93 \%)$ and Eurasian $(224,17.43 \%)$ plants (16.99 \%) (Tab. 7). This was expected due to the geographical and the phytogeographical position of the mountain. The influence of the Mediterranean climate on Svilaja is less pronounced than on Mosor and Šibenik, which is reflected in the significantly lower contribution of the Mediterranean geoelement (Tab. 8). On the other hand, the presence of widespread plants (14\%), and cultivated and alien plants (7.24\%) may be used as an indicator of the intensity of human influence. These plant species were less common in the higher altitudes and uninhabited areas than on the lower slopes of Svilaja where the anthropogenic impact is more pronounced. 
Tab. 7. Analysis of the geoelements on the Mt Svilaja

\begin{tabular}{|c|c|c|}
\hline Geoelement & No. of taxa & $\%$ \\
\hline MEDITERRANEAN & 299 & 23.27 \\
\hline Circum-Mediterranean plants (ME-circ) & 114 & 8.87 \\
\hline West Mediterranean plants (ME-west) & 7 & 0.54 \\
\hline East Mediterranean plants (ME-east) & 18 & 1.40 \\
\hline Illyrian Mediterranean plants & 106 & 8.25 \\
\hline Illyrian-South European plants (ME-ilseu) & 19 & 1.48 \\
\hline Illyrian-Adriatic plants & 87 & 6.77 \\
\hline Illyrian-Adriatic endemic plants (ME-ilade) & 70 & 5.45 \\
\hline Illyrian-Apennine plants (ME-ilape) & 17 & 1.32 \\
\hline Mediterranean-Atlantic plants (ME-atl) & 26 & 2.02 \\
\hline European Mediterranean plants (ME-eu) & 11 & 0.86 \\
\hline Mediterranean-Pontic plants (ME-po) & 17 & 1.32 \\
\hline ILLYRIAN-BALKANIC & 51 & 3.97 \\
\hline Balkanic-Apennine plants (ILBA-baap) & 8 & 0.62 \\
\hline Illyrian-Balkanic endemic plants (ILBA-end) & 43 & 3.35 \\
\hline SOUTH EUROPEAN & 269 & 20.93 \\
\hline South European-Mediterranean plants (SEU-me) & 159 & 12.37 \\
\hline South European-Pontic plants (SEU-po) & 61 & 4.75 \\
\hline South European-mountain plants (SEU-mo) & 31 & 2.41 \\
\hline South European-continental plants (SEU-co) & 9 & 0.70 \\
\hline South European-Atlantic plants (SEU-atl) & 9 & 0.70 \\
\hline EAST EUROPEAN-PONTIC (EEUPO) & 24 & 1.87 \\
\hline SOUTHEAST EUROPEAN (SEEU) & 24 & 1.87 \\
\hline CENTRAL EUROPEAN (CEU) & 32 & 2.55 \\
\hline EUROPEAN (EURO) & 53 & 4.12 \\
\hline EURASIAN (EUAS) & 224 & 17.43 \\
\hline CIRCUM-HOLARCTIC PLANTS (CIHO) & 37 & 2.88 \\
\hline WIDESPREAD PLANTS (WISP) & 179 & 13.93 \\
\hline CULTIVATED \& ALIEN PLANTS (CUAP) & 93 & 7.24 \\
\hline TO T A L & 1285 & 100.00 \\
\hline
\end{tabular}

Tab. 8. Percentage contribution of the geoelements in the flora of Mt Svilaja comparison to that of Mt Mosor (Vladović, 1994), Šibenik and its surroundings (Milović, 2002) and Mt Papuk (PandžA, 2010).

\begin{tabular}{|c|c|c|c|c|c|c|}
\hline \multirow{2}{*}{ Area } & \multicolumn{7}{|c|}{ Geoelement (\%) } \\
\cline { 2 - 7 } & Mediterranean & S European & Eurasian & European & Widespread & $\begin{array}{c}\text { Cult. \& } \\
\text { alien }\end{array}$ \\
\hline $\begin{array}{c}\text { Šibenik and } \\
\text { surroundings }\end{array}$ & 37.86 & 20.65 & 10.33 & 3.44 & 15.53 & 8.09 \\
\hline Mt Mosor & 32.91 & 22.02 & 12.15 & 3.78 & 13.42 & 7.57 \\
\hline Mt Svilaja & 23.27 & 20.93 & 17.43 & 4.12 & 14.00 & 7.24 \\
\hline Mt Papuk & 1.96 & 17.83 & 27.39 & 12.18 & 17.58 & 6.54 \\
\hline
\end{tabular}




\section{Endemic, endangered and protected plant taxa}

In total, 202 taxa (15.72\%) belong to at least one of the groups of special interest for conservation (endemics, endangered or strictly protected taxa, sensu Croatian law). There are no real mountain endemics. Seventy-six taxa $(5.91 \%)$ were considered to be endemic (Tab. 9, sensu Nikolić et al., 2015), mostly belonging to the group of Illyrian-Adriatic endemics. Croatian endemic taxa presented on Svilaja are: Arenaria orbicularis Vis., Iris adriatica Trinajstić ex Mitić, Knautia adriatica Ehrend, K. dalmatica Beck, Ophrys dinarica Kranjčev \& P.Delforge, O. liburnica Devillers \& Devillers-Tersch., O. rhodostephane Devillers \& Devillers-Tersch., etc.

Tab. 9. Number of taxa of special interests for conservation in Croatia found on Mt Svilaja

\begin{tabular}{|l|c|c|}
\hline \multicolumn{1}{|c|}{ Category } & No. of taxa & \% of total no. of taxa \\
\hline Endemics (end; sensu Nikolić et al., 2015) & 76 & 5.91 \\
\hline Critically Endangered (CR) & 1 & 0.08 \\
\hline Endangered (EN) & 8 & 0.62 \\
\hline Vulnerable (VU) & 25 & 1.95 \\
\hline CR+EN+VU & 34 & 2.65 \\
\hline Near Threatened (NT) & 33 & 2.57 \\
\hline Data Deficient (DD) & 38 & 2.96 \\
\hline Least Concern (LC) & 21 & 1.63 \\
\hline NT + DD + LC & 92 & 7.16 \\
\hline Croatian strictly protected (sp) & 155 & 12.06 \\
\hline
\end{tabular}

The contribution of endemics on Svilaja may be compared to that on Matokit $(5.30 \%)$, but it is lower than in Paklenica National Park $(9.77 \%)$ in the southern Velebit (Alegro, 2004).

Among the endemics, the presence of Scabiosa delminiana Abadžić should be stressed. This plant was recently described as new for science on Mt Lip (Lib) on the edge of Duvanjsko Polje in neighboring Bosnia and Herzegovina (ABADžıć, 2007). Until now, the only known locality in Croatia so far was along the Butižnica River, a tributary of the Krka River (ABADŽIĆ, 2007; Nıkolić et al., 2014). We found a large population with several hundred individuals on the sub-Mediterranean montane calcareous rocky grasslands on shallow soils of the alliance Saturejion subspicatae Tomić-Stanković 1970, on the southeastern slopes on the locality of Orlove Stine (x = 4848222, $y=6381723$ ) not far from the hiking lodge.

Further, the endemic taxon Hyacinthella dalmatica (Baker) Chouard, common in Dalmatia, is abundant on rocky and hilly grasslands on the northern (Lemeš, Maovice, Vrlika) and the southern slopes (Ogorje, Zelovo) of Svilaja.

In total, 34 taxa $(2.66 \%)$ are included in the Croatian Red List (Nikolić \& Topić, 2005, Nikolić, 2020b). One species (Vaccaria hispanica (Miller) Rauschert) is Critically Endangered (CR). Eight taxa (e.g., Delphinium peregrinum L., Hibiscus trionum L., Hordeum secalinum Schreb., Ophrys apifera Huds., etc.), are considered Endangered (EN), while 25 are classified as Vulnerable (VU), i.e., Alopecurus rendlei Eig., Arctostaphylos uva-ursi (L.) Spreng., Ilex aquifolium L., Lilium carniolicum Bernh. ex W.D.J.Koch, 
Ophrys bertolonii Moretti, Orchis militaris L., O. simia Lam., Platanthera bifolia (L.) Rich., Polygonatum latifolium (Jacq.) Desf., etc.

In total, 92 taxa have been classified as Nearly Threatened (NT), Least Concern (LC), or Data Deficient (DD) (Nikolić \& Topić, 2005, Nikolić, 2020b). Some of these taxa are Hymenolobus procumbens (L.) Nutt., Hyssopus officinalis L., Polycnemum arvense L., Pulsatilla grandis Wender., Ruscus aculeatus L., Teucrium arduini L., Tulipa sylvestris L., etc.

In total, 155 taxa $(12.06 \%)$ found on Svilaja are strictly protected in Croatia (ANONYMous, 2013). This also includes all endemics, endangered taxa, orchids (Orchidaceae), and taxa from the genera Dianthus and Iris, etc.

Svilaja is characterized by a rich orchid flora containing 51 taxa $(3.97 \%)$ and includes several endemic taxa of the genus Ophrys: O. dinarica Kranjčev \& P.Delforge, O. liburnica Devillers \& Devillers-Tersch., O. rhodostephane Devillers \& Devillers-Tersch., O. sphegodes Mill. subsp. tommasinii (Vis.) Soó, O. tetraloniae W.P.Teschner and O. untchiii (M.Schulze) P.Delforge. The high number of orchid taxa found on Svilaja, especially on its southwestern slopes in the vicinity of Muć, has also been pointed out by several authors (Golubić, 1999, 2001; KranjČev, 2005; Delforge, 2006; Milović et al., 2013; Š EGOTA, 2016). Rocky dry grasslands and open forests that occupy relatively large areas on Svilaja represent favourable habitats for various orchid species. The taxa Ophrys annae Devillers-Tersch. \& Devillers, O. gracilis (Büel, O.Danesch \& E.Danesch) Englmaier and O. serotina Rolli ex Paulus, reported for Svilaja (Muć, Postinje) by KranjčEv (2005) are designated as doubtful in FCD (Nikolić, 2020).

Delforge (2006a) visited several localities on Svilaja in the period from 2002 to 2006, and concluded that (i) O. annae, O. gracilis, and O. serotina do not occur in Croatia, (ii) the findings of KRANJČEV (2005) published under the above mentioned names have to be attributed to the taxon O. tetraloniae, (iii) two hybrids also grow on Svilaja: Ophrys $\times$ kranjcevii P.Delforge $(O$. dinarica $\times$ O. untchjii) in Karakašica, and Cephalanthera $\times$ schulzei E.G.Camus, Bergon, A.Camuis $(C$. damasonium $\times$ C. longifolia) in Zelovo, (iv) of particular importance is the record of Epipactis placentina Bongiorni \& Grünanger from Zelovo (site 262, XJ2344, $780 \mathrm{~m}$ a.s.1.). This is the first record of $E$. placentina for Croatia and needs to be included in the FCD. We found a population of about 100 individuals of E. placentina in the forest near Gornje Ogorje, south of the settlements of Šunjići and Jelavići $(x=4844918, y=6382266)$.

\section{Rare species of Croatian and Dalmatian flora on Svilaja}

This section reports the records of several rare taxa either for a whole Croatian territory or for Dalmatia with the aim of contributing to the current state of knowledge of their distribution range (for details on the previous distribution range for each taxon see FCD; Nikolić, 2020a).

Achnatherum calamagrostis (L.) P.Beauv. (Poaceae) is a South European-montane taxon, known only in a few localities in Croatia: in the northern part of the Krka National Park (Grčići), next to Krčić waterfall, the spring of the Una River, on Mt Dinara (Brezovac) and on the island of Hvar. On Svilaja, it occurs along the edges of the mountain road south of the Orlove Stine hiking lodge $(x=4847487, y=6381670 ; x$ $=4847029, \mathrm{y}=6381313)$. 
Carlina acanthifolia All. (Asteraceae) occurs predominantly in the mountainous areas of southern and eastern Central Europe. It is relatively rare in Croatia (Istria, Gorski Kotar, Lika, Nova Gradiška in Slavonia). The records on Svilaja (Lemeš, Razvale Maovičke, Orlove Stine) are currently the southernmost in Croatia.

Delphinium fissum Waldst. \& Kit. (Ranunculaceae) is a Eurasian species that grows on mountain pastures. In Croatia, it is known only at a few sites: Paklenica, Poljica Kozička and Mosor. Several individuals were found on the grasslands on the southern slopes of Orlove Stine.

Dianthus carthusianorum L. subsp. sanguineus (Vis.) Hegi (Caryophyllaceae) is rare in terms of the number of localities in Croatia. It is found in Istria, Samobor Hills and at several sites in Lika. In Dalmatia, the only record originated from Mt Biokovo (RADIć, 1976). In this study, we found it in Gornje Ogorje (between Tešije and Jeličići).

Erodium acaule (L.) Bech. \& Thell. (Geraniaceae) is a Mediterranean-montane plant species that was recently included in Croatian flora based on findings from southern Istria and the island of Krk (Topić et al., 1998). It was later found in the vicinity of Bibinje and on the island of Silba, northern Dalmatia (BogDANović et al., 2013), but it probably has a wider distribution in the coastal area of Croatia. Some authors have so far not distinguished it from the morphologically similar E. cicutarium (L.) L' Hér. This therophyte grows mostly as a weed in crops, while E. acaule occurs on dry places, mostly in rocky grasslands and garrigues (Topıć et al., 1998). On Svilaja, we found it in several localities on dry grasslands near Lemeš, next to the Sv. Jure hiking lodge, in Maovice (between Režići and Baturine), Maljkovo and near the settlement of Vrba.

Euphorbia barrelieri Savi subsp. hercegovina (Beck) Kuzmanov (Euphorbiaceae) is a subspecies endemic to Croatia, Bosnia and Herzegovina, and Montenegro. Its taxonomic status with respect to the morphologically similar E. triflora Schott, Nyman \& Kotschy has not yet been fully resolved (JASPRICA et al., 2009; Nikolić et al., 2014; FrAJMAN \& SCHÖNSWETTER, 2017). In Croatia, it was known only from sites in the vicinity of Cavtat and on Pelješac Peninsula. The record from Svilaja originated from the area of Lučani ( $\mathrm{x}=4843059, \mathrm{y}=6387053)$.

Gentiana dinarica Beck (Gentianaceae) is endemic to the Dinarides (Croatia, Bosnia and Herzegovina, Montenegro). It occurs on alpine and subalpine calcicolous swards (ŠILIĆ, 1984). The only record for Croatia originated from the southwestern slopes of Svilaja, above the settlements of Ogorje and Zelovo (Š́GOTA, 2016). Additionally, we found a larger population of G. dinarica on rocky grassland in the vicinity of the hiking lodge below Orlove Stine $(x=4848174$, y = 6381626).

Gypsophila fastigiata L. (Caryophyllaceae) is a central and eastern European species that favours continental ridged forests, sandy soils and calcareous rocks. In Croatia, it is found on the southern border of its distributional range, but known only at a few sites: Mt Kalnik and North Velebit National Park. On Svilaja, it was found on dry grasslands in several places south of Orlove Stine $(x=4847991, y=6381653 ; x=$ 4847024, y =6381289).

Ilex aquifolium L. (Aquifoliaceae) is an endangered (VU) taxon in Croatia. It grows in beech forests. In Svilaja, several individuals were found in a beech forest near Sutina $(x=4841226, y=6384924)$. This is the only record in Dalmatia and the southernmost locality for Croatia. TAFRA et al. (2012) reported this species from cultivation in Omiš. 
Iris adriatica Trinajstić ex Mitić (Iridaceae) is an endemic taxon for the coastal area and islands in northern and central Dalmatia. On Svilaja, a population of a hundred individuals has been found on rocky grassland, along the Drniš-Vrlika road, near Štikovo $(x=4861624, y=5604635)$. This is the locality the most remote from the Adriatic coast.

Knautia adriatica Ehrend. and K. dalmatica Beck (Dipsacaceae) are Croatian endemics from Dalmatia. Knautia adriatica occurs in the vicinity of Zadar and Biograd and in the hinterland of Šibenik (Trtar), while K. dalmatica grows on Kamešnica, Mosor, Svilaja, Kozjak and the island of Brač (BogDAnović, 2015b). The area of Svilaja represents the boundary between the ranges of these two species. A small population of K. adriatica was found on the rocky grassland in Ogorje (Kokani) next to wind turbines ( $\mathrm{x}=$ $4842708, \mathrm{y}=6381648)$ and in the vicinity of the Golubinka pit $(\mathrm{x}=4847265, \mathrm{y}=$ 5621393). Several individuals of $K$. dalmatica were found in the vicinity of Cavoglave $(\mathrm{x}=4849815, \mathrm{y}=5607759)$ and along the hiking trail east of Crni Umac.

Knautia clementii (Beck) Ehrend. (Dipsacaceae), endemic to the central Dinarides, occurs in Croatia and Bosnia and Herzegovina. In Croatia, it has been recorded at several localities on central and southern Velebit, on Dinara and near Vrlika (Ježević) (Bogdanović, 2015a). Specimens of this plant species were collected on a hilly grassland between Ogorje and Zelovo.

Knautia travnicensis (Beck) Szabó (Dipsacaceae) is endemic to the central Dinarides (Croatia and Bosnia and Herzegovina). In Croatia, it is known for several localities on Velebit, Dinara and Plješivica. On Svilaja, a new locality for Croatia was discovered between Ogorje and Zelovo.

Lamium bifidum Cirillo (Lamiaceae) is a eu-Mediterranean taxon, found in Croatia only at a few localities: Trogir and Svilaja (VIsiani, 1847: 211), Seoci and Kotlenice on Mosor (Vladoović \& Iljuanić, 1993), and Sutina on Svilaja (Vladović et al., 2019). According to the presence of several images in FCD (uncoded data), the species was also found in the island of Brač and vicinity of the town of Cavtat in South Dalmatia. We found several individuals in the vicinity of Raketići stables $(x=4859944, y=$ 5602552).

Lathraea squamaria L. (Scrophulariaceae) is a species of Eurasian distribution that parasitizes on the roots of deciduous trees (PignATTI, 1982). In Croatia, it is distributed in continental part of the country, and in Trilj, which is the only site in Dalmatia. We found several individuals in Vrlika, in the forest near the Česma spring (Vrilo).

Linum hirsutum L. (Linaceae) is a central-eastern European - Pontic species (PIGNATTI, 1982). It is known from several localities in continental Croatia, while in the coastal part of Croatia it has been recorded only in two areas in Dalmatia: on Hrvatačko Polje and Pelješac Peninsula. We found a large population on the grasslands along the road through Lučane.

Lomelosia graminifolia (L.) Greuter \& Burdet (Dipsacaceae) is a southern European species of colline and montane areas (PignatTi, 1982). In Croatia, it has been recorded in Gorski Kotar, Velebit, Plješivica and Biokovo. On Svilaja, it is widespread on limestone grasslands. We found it in several localities, especially along the hiking trail from Ogorje to Orlove Stine.

Moenchia mantica (L.) Bartl. (Caryophyllaceae) is a northern Mediterranean species (PIgnatti, 1982). It is widespread in continental Croatia, but rare in the coastal area. It 
was found on Dinara (Trinajstić \& Šugar, 1972), Mosor (Vladović \& Iljuanić, 1992) and on the island of Vis (Domac, 1955). We found it at two sites on Svilaja, in Maovice (between Režići and Baturine) and Zelovo (Lađa).

Nigella arvensis L. (Ranunculaceae) grows most commonly in cereal fields. Most of the sites were found in continental Croatia, but they are rare in the Croatian coastal area: Donji Kamenjak (Istria), Šibenik, Solin, Hrvatačko Polje, the islands of Brač, Korčula and Mljet, and Trsteno near Dubrovnik. It was once widespread in Dalmatia (VISIANI, 1852; "in agris et satis totius Dalmatiae"), but due to the abandonment of areas of cereal cultivation it became very rare. We found it on the western slopes of Svilaja (Gradac).

Odontites vulgaris Moench (Scrophulariaceae) is a Eurasian species common in continental Croatia and in the northern Adriatic, and it is very rare in Dalmatia: Krka National Park (Visovac) and Split. Previously reported data for Zadar, Drniš, and Dubrovnik (VISIANI, 1847: 174) have not been confirmed in recent times. The species has already been reported for Sutina (Vladović et al., 2019), and we found it on the southwestern slopes of Svilaja, in Gradac and Mirlović Polje (Vrba, Čikola).

Onobrychis alba (Waldst. \& Kit.) Desv. (Fabaceae) is a circum-Mediterranean species. In Croatia, only a typical subspecies is present, and in only a few localities: Zagreb (Maksimir), Risnjak, Kozjak, Mosor, Biokovo and Snježnica (Konavle), and on the island of Čiovo. Several individuals were found in Gornje Ogorje (Kokani) and in the vicinity of Grabići.

Onopordum acanthium L. (Asteraceae) is a Eurasian species, relatively rare in continental Croatia, and even rarer along the Adriatic coast and the islands. In Dalmatia, it is known only from a few localities in the Krka National Park, Cetinsko Polje and Sinjsko Polje. The records from Drniš and the island of Hvar (VISIANI, 1847: 45) have not been recently confirmed. In this study, it is found in the several localities: Vrlika, Otišić, Maljkovo, Donje Ogorje, Bidnići and Gornje Ogorje (Kokani). This study confirmed the findings of VISIANI (1847: 45), from ruderal habitats on the hills around Sinj.

Oxytropis dinarica (Murb.) Wettst. (Fabaceae) is endemic to the Dinarides. In Croatia, it is distributed on Risnjak, Velebit, Poštak, Dinara and Biokovo. In this study, $O$. dinarica was found on meadows in the highest altitudes as Lovrić et al. (1989) have previously reported.

Pedicularis brachyodonta Schloss. \& Vuk. (Scrophulariaceae) is a taxon distributed in the northern and central parts of the Balkans. In Croatia, it is known on Klek (near Ogulin), Velebit, Šatorina and Plješivica. Lovrić et al. (1989) reported the species from Svilaja and Veliki Kozjak, but we could not confirm their finding.

Petteria ramentacea (Sieber) C.Presl (Fabaceae) is an Illyrian-Balkan endemic species. In Croatia, it is found in central and southern Dalmatia. Lovrić et al. (1987) reported it from the "southern dolomite slopes of Svilaja towards Muć and Dicmo". In this study, its presence was not confirmed.

Rapistrum rugosum (L.) All. subsp. rugosum (Brassicaceae) is a species of circum-Mediterranean distribution. On the species level (R. rugosum), it was recorded in Istria and the Kvarner islands, while records from Dalmatia seem to be rare and mostly originated from the $19^{\text {th }}$ century (VISIANI, 1852: 105). On the subspecies level, it 
is known only from a few localities in Istria. The locality on Svilaja (Otavice) is only one outside of Istria.

Rubus candicans Weihe ex Rchb. (Rosaceae) is distributed in southern, western and central Europe. It is rare in Croatia, found mostly in the western part of the country and in Istria (Učka). On Svilaja, it was found only in the area of Samar.

Sambucus racemosa L. (Caprifoliaceae) is a circumboreal species that occurs mainly in the highest mountain forests. In Croatia, it is distributed in the area of Učka, Gorski Kotar, Velebit and Lika. In Dalmatia, it was recorded on Dinara and Pelješac Peninsula. On Svilaja, we recorded it in a burnt beech forest in Samar.

Scrophularia bosniaca Beck (Scrophulariaceae) is endemic to the southeastern Dinarides (Croatia, Bosnia and Herzegovina, Montenegro, Kosovo and Albania), and is mostly found in beech forests, rock crevices and ravines (ŠIlić, 1984). Until now, it was found on Dinara, while on Svilaja it was observed in the rock crevices of Lisina and Samar.

Seseli elatum L. subsp. gouanii P.W.Ball (Apiaceae) is endemic to northern Italy and the western part of the Balkans (Slovenia, Croatia and Bosnia and Herzegovina). In Croatia, the largest number of localities was observed in Hrvatsko Primorje and on Velebit, and others were also found in Istria (Ćićarija), Gorski Kotar and Gola Plješivica in Lika (Milović, 2015). The finding on Svilaja (Orlove stine) is the only one in Dalmatia.

Silene sendtneri Boiss. (Caryophyllaceae) is endemic to the Dinarides, known in Croatia for only a few localities in Gorski Kotar, Plitvice Lakes and Dinara. Lovrić et al. (1989) reported it, without specifying precise localities, for Veliki Kozjak and Svilaja. We found this taxon on a hilly meadow below Orlove stine (ca. $1000 \mathrm{~m}$ a.s.1.).

Stachys thirkei K.Koch (Lamiaceae) is distributed in the northeastern Mediterranean. In Croatia, the most localities were found in the Krka River Canyon, and others were also reported from Kvarner in the north to Biokovo in the south. On Svilaja, the species is very frequent (found in 18 localities) at lower altitudes. This species probably has a wider distribution in Croatia, and in some areas (Zadar, Split, Šibenik) was previously replaced by the closely related S. cretica L. subsp. salviifolia (Ten.) Rech.f.

Tragopogon balcanicus Velen. (Cichoriaceae) occurs in the northern and central Balkans. In Croatia, it was found in southern Velebit, near Šibenik, on Mosor and Biokovo, and in the Tijarica valley. On Svilaja, it was found at several localities: Čavoglave, Polje, Samar, Potravlje (Babići) and Gornje Ogorje (between Tešije and Jeličići).

Typha domingensis (Pers.) Steud. (Typhaceae) is a species of the temperate and tropical regions. In Croatia, it is known only from the island of Cres and from Lake Prokljan, the Krka River (Marković et al., 1993). We believe that it has a wider distribution in the coastal part of Croatia where it is probably confused with a similar taxon $T$. angustifolia L. Cook (1980: 276) states that some of the records of T. angustifolia in South Europe perhaps refer to T. domingensis. On Svilaja, this taxon was found at several localities: Otavice, the confluence of the Vrba in Cikola, Gornje Polje (Crivac), Radunić, the Suvova stream near Muć, Gornje Ogorje (a pond near village of Kokani). Vladović et al. (2019) recorded T. angustifolia for Sutina, and according to our opinion, this record is doubtful and may also be attributed to T. domingensis.

Vincetoxicum fuscatum (Hornem.) Rchb.f. (Asclepiadaceae) is a Southeast European species that grows in rocky dry habitats (MARKGRAF, 1972). In Croatia, only a few 
localities have been recorded for the islands of Brijuni, Cres, Krk, Lošinj and Hvar, and Śćadin (Blato na Cetini). On Svilaja, the species was found in Orlove Stine, Gornje Ogorje (Tešija) and along the hiking trail between Jačmenjaci and Gole Kose.

\section{Cultivated, alien and invasive species}

In total, 93 cultivated and adventitious plants taxa (7.24\%) were found. This is in accordance with the data obtained for Mosor and Papuk (Tab. 8), but significantly lower than in the urban areas along the Croatian coast (RuščIć, 2002; TAFrA et al., 2012, Milović \& Mitić, 2012). Cultivated and alien plants are mainly related to ruderal and weed vegetation in settlements on the lower altitudes on Svilaja, while they are almost completely absent in the higher vegetation belts.

The most common were alien species - intentionally (for cultivation) and unintentionally introduced species with different degrees of domestication (sensu RICHARDSON et al., 2000; PYŠEK, 2004). Among them, special attention was paid to invasive taxa that can cause disturbances in the phytocoenosis. In total, 37 taxa $(2.88 \%)$ were considered invasive (for details see Boršić et al., 2008, and Nikolić et al., 2014) on Svilaja. The most invasive taxa were found in one to three localities only: e.g., Acer negundo L., Amaranthus albus L., A. blitoides S.Watson, A. hybridus L., Ambrosia artemisiifolia L., Amorpha fruticosa L., Artemisia annua L., A. verlotiorum Lamotte, Bidens subalternans DC., Chamomilla suaveolens (Pursh) Rydb., Conyza bonariensis (L.) Cronquist, Cuscuta campestris Yunck., Datura wrightii Regel, E. prostrata Aiton, Juncus tenuis Willd., Panicum capillare L., Paulownia tomentosa (Thunb.) Steud., Sorghum halepense (L.) Pers., and Xanthium spinosum L. The findings of the Reynoutria japonica Houtt. (Gornje polje, Crivac) and Solidago gigantea Aiton (Gradac), quite rare taxa along the Croatian coastal area, but widespread in the continental area of Croatia (Nikolić, 2020a), were of particular interest. The most frequent invasive species on Svilaja were: Ailanthus altissima (Mill.) Swingle, Amaranthus retroflexus L., Broussonetia papyrifera (L.) Vent., Conyza canadensis (L.) Cronquist, Erigeron annuus (L.) Pers., Helianthus tuberosus L., Robinia pseudoacacia L., and Veronica persica Poir. Additionally, Marković (1964) recorded an invasive species Lepidium virginicum $\mathrm{L}$. within the ruderal vegetation in Vrlika, but the record was not confirmed in our study.

However, this study showed that distribution of aliens is localized in the anthropogenic habitats in settlements and its surroundings and, at this moment, does not pose a threat to natural habitats.

In the settlements on Svilaja, numbers of horticultural species with the ability to spread outside cultivation were recorded. They were: Alcea rosea L., Aster lanceolatus Willd., Calendula officinalis L., Campsis radicans (L.) Seen., Fallopia baldschuanica (Regel) Holub, Gaillardia aristata Pursh., Ipomoea purpurea Roth, Lilium candidum L., Mirabilis jalapa L., Oxalis articulata Savigny, Paulownia tomentosa (Thunb.) Steud., Tagetes patula L., etc. Among them, two ornamental plant species need to be highlighted: (i) Hemerocallis fulva (L.) L., already known from several localities in Croatia (Nıkolić, 2020a), is distributed in cultivation over a large area in settlements on Svilaja. It was also found outside cultivation in Miočić, Gradac (Vidovići), between Polje and Čavoglave, Crivac, Satrić, Muć (near Suvova stream) and Karakašica; (ii) Oenothera speciosa Nuttall is also found in Miočić. The species has to be included in FCD as a new member of Croatian flora found for the first time outside the culture. 


\section{CONCLUSIONS}

The study provides a comprehensive floristic list of the Mt Svilaja for the first time. Mt Svilaja hosts a high number of vascular plant taxa and this confirms its position within the Western Balkans as a high biodiversity hotspot. This moderately high limestone mountain located in the transitional zone between the alpine-high-Nordic and the oro-Mediterranean floristic regions (sensu STEvanović, 1996) has a humid climate with warm summers and covers a high altitude range. All these, including a long-term human impact, account for the mountain being an excellent reservoir of biodiversity, and particularly of orchids. It has a considerable proportion of Croatian endemics, and a high capacity to host important species from a conservation point of view, while having a low number of invasive plant species.

The transitional position between two floristic and vegetation (Mediterranean and Euro-Siberian) regions is also confirmed by the analysis of the life-forms and geoelements. The domination of the Mediterranean geolement can be explained by the maritime impact which results in penetration of Mediterranean species deep into the hinterland through the Cetina River Canyon (e.g., AnTonić \& Lovrić, 1996).

\section{ACKNOWLEDGEMENTS}

We thank our colleagues Sandro Bogdanović and Ivana Rešetnik for their help in the identification of Knautia; Jagoda Karađole and Robert Crnković for their help in finding the localities of several rare species on Svilaja. We also thank the reviewers for their careful reading of the manuscript and constructive suggestions.

Received October 5, 2020

\section{REFERENCES}

AвaDžıć, S., 2007: Nova vrsta genusa Scabiosa L. (Dipsacaceae). Hrvatska misao XI, 1/07(42), n. s. sv. 30, 38-49.

Adamović, L., 1911: Die Pflanzenwelt Dalmatiens. Werner Klinkhardt, Leipzig.

Adamović, L., 1929: Die Pflanzenwelt der Adrialänder. Gustav Fischer, Jena.

Alegro, A., 2004: Biljni svijet NP "Paklenica". Paklenički zbornik 2. Povodom 55. godišnjice Nacionalnog parka "Paklenica". Starigrad-Paklenica, 35-54.

Antonić, O. \& A.Ž. Lovrić, 1996: Numerical analysis of vegetation complexes and community diversity of major coastal Dinaric mountains. J. Veg. Sci., 7, 73-80.

Anonymous, 2013: Pravilnik o strogo zaštićenim vrstama, NN 144/2013, Prilog I.

BARIĆ, LJ., 1957: Eruptivi iz okolice Sinja u Dalmaciji, uz kraći osvrt na eruptivne pojave kod Knina, Vrlike i Drniša. Zbornik 2. kongresa geologa Jugoslavije, Sarajevo, 255-262.

Bogdanović, S., 2015a: Asperula staliana Vis. U: Nikolić, T., Milović, M., Bogdanović S. \& Jasprica, N., Endemi u hrvatskoj flori, Alfa d.d., Zagreb, 70-71.

Bogdanović, S., 2015b: Knautia adriatica Ehrend., K. clementi (Beck) Ehrend., K. dalmatica Beck. In: Nikolić, T., Milović, M., Bogdanović S. \& Jasprica, N., Endemi u hrvatskoj flori, Alfa d.d., Zagreb, 302-306.

Bogdanović, S., Župan, D. \& Mitić, B., 2013: Vaskularna flora otoka Silbe. In: Mužinić, J. \& Purger, J.J. (eds.), Otok Silba, prirodno i kulturno blago. Sveučilište u Zadru, 81-94.

Bogunović, M. \& Bensa, A., 2006: Tla krša - temeljni čimbenik biljne proizvodnje. In: Biondić, B. \& BožıčEvić, J. (eds.), Hrvatski krš i gospodarski razvoj, Centar za krš, Zagreb, 41-50. 
BojAnić, L., 1961: Allgemeiner geologischer Aufbau des Hauptkammes der Svilaja. Bull. Int. Acad. Yougoslave Sci., Cl. Sci. Math., 6(4), 100.

Boršić, I., Milović, M., Dujmović, I., Bogdanović, S., Cigić, P., Rešetnik, I., Nikolić, T. \& Mitić, B., 2008: Preliminary check-list of invasive alien plant species (IAS) in Croatia. Nat. Croat., 17(2), $55-71$.

Brkić, Ž., Biondić, R., Pavičić, A., Slišković, I. Marković, T., Terzić, J., Dukarić, F. \& Dolić, M., 2006: Određivanje cjelina podzemnih voda na Jadranskom slivu prema kriterijima Okvirne direktive o vodama EU. Arhiv Hrvatski geološki institut, Zagreb.

Cook, C.D.K., 1980: Typha L. In: Tutin, T.G., Heywood, V.H., Burges, N.A., Moore, D.M., Valentine, D.H., Walters, S.M. \& Webв, D.A., (eds.): Flora Europaea 5. University Press, Cambridge, 275276.

Čović, A., 2017: Florističke i meliorativne značajke šumskih kultura crnog bora (Pinus nigra J. F. Arnold) na području šumarija Drniš i Split. Šumarski fakultet Sveučilišta u Zagrebu, Završni specijalistički rad, 1-115.

Degen, A., Janchen, E. \& Watzl, B., 1908: Ein Beitrag zur Kenntnis der flora der Dinarischen Alpen. Oesterr. Bot. Z. 58(3, 4, 5, 6/7, 8, 9, 10). Wien.

Delforge, P., 2006a: Contribution à la connaissance des Orchidées de Croatie. Résultats de cinq années de prospections. Natural. Belges., 87 (Orchid. 19), 141-200.

Delforge, P., ed., 2006b: Orchids of Europe, North Africa and the Middle East (2nd ed.), English translation, A. \& C. Black, London.

Delić, A., 2010: Novi nalazi endemičnog leptira dalmatinskog okaša Proterebia afra dalmata (Godart, 1824) (Lepidoptera, Satyrinae) u Dalmaciji (Hrvatska). In: MATAS, M. \& FAričić, J. (eds.): Zagora između stočarsko-ratarske tradicije te procesa litoralizacije i globalizacije: knjiga sažetaka / Znanstveni skup s međunarodnim sudjelovanjem, Zadar - Dugopolje, 19-21. listopada 2010, 13.

Domac, R., 1955: Flora otoka Visa. Acta Pharm. Jugosl., 1, 3-43.

Domac, R., 1994: Flora Hrvatske. Priručnik za određivanje bilja. Školska knjiga, Zagreb.

Durbešić, A. \& Milković, I., 2005: Pošumljavanje neobraslog šumskog zemljišta na južnim padinama Svilaje-Muć s ciljem protuerozijskog djelovanja. Zbornik radova međunarodnog znanstvenog skupa "Protuerozijska i vodozaštitna uloga šume i postupci njezina očuvanja i unapređenja", Šumar. List, 129 (Suppl. 1), 133-143.

Durbešıć, A. \& Fuerst-BJelıš, B., 2016: Tipovi i trendovi promjene pejzaža planine Svilaje - Ogorje (Types and trends in landscape changes at Svilaja - Ogorje mountain area), Ekonomska i Ekohistorija, 12(12), 208-221.

Frajman, B. \& Schönswetter, P., 2017: Amphi-Adriatic distributions in plants revisited: Pleistocene trans-Adriatic dispersal in the Euphorbia barrelieri group (Euphorbiaceae). Bot. J. Linn. Soc., 185(2), 240-252.

Golubić, V., 1999: Izložba slika. Orhideje (Orchidaceae) od Kaštela do planine Svilaje. Katalog izložbe. Split.

Golubić, V., 2001: Izložba fotografija. Orhideje Dalmatinske zagore (Orchids of the Dalmatian Zagora). Katalog izložbe. Split.

Horvat, I., 1949: Nauka o biljnim zajednicama. Nakladni zavod Hrvatske, 1-434.

Horvatić, S., Ilijanić, Lj. \& Marković-Gospodarić, Lj., 1967-1968: Biljni pokrov okolice Senja, Senjski zbornik, 3, 298-322.

JASPRICA, N., RuŠčić, M. \& Kovačıć, S., 2009: Fitosociološka pripadnost vrste Euphorbia hercegovina G. Beck u Hrvatskoj. Zbornik sažetaka 10. Hrvatskog biološkog kongresa s međunarodnim sudjelovanjem, 14. - 20. 09. 2009., Osijek, 136-137.

Janchen, E., 1908: Eine botanische Reise in die Dinarischen Alpen und den Velebit. Mitt. Naturwiss. Ver. an der Univ. Wien, 6, 69-97.

Janchen, E., 1910: Die Edraianthus-Arten der Balkanländer. Mitt. Naturwiss. Ver. an der Univ. Wien, $8,1-40$.

Jedlovski, D., 1952: Prilog istraživanju areala bukve u Dalmaciji. Šumar. List, 76(5-6), 160-164.

Köppen, W. \& R. Geiger, 1954: Klima der Erde. Darmstadt: Justus Perthe.

Kamenjarin, J., 1996: Vascular flora of mount Kozjak above Split. Nat. Croat., 5(2), 119-144. 
KRANJČEv, R., 2005: Hrvatske orhideje: prilozi za hrvatsku floru: staništa, svojte, hibridi, zaštita. AKD Zagreb, 1-518.

Kranjčev, R. \& ŠĚšk, D., 2016: A revision of the genus Fritillaria (Liliaceae) in Croatia. Nat. Croat., 25(2), 185-212.

KušAn, F., 1969: Biljni pokrov Biokova (Flora i vegetacija). Prirodoslovna istraživanja JAZU, 37 (Acta Biol. V), Zagreb, 5-187.

Lovrić, A. Ž. \& RAC, M., 1989: Reliktna visokoplaninska vegetacija najhladnijih vrhova na južnim primorskim Dinaridima i njezino paleogeografsko porijeklo. Acta Biokovica, 5, 131-148.

Lovrić, A. Ž., Rac, M., Bedalov, M. \& Š Egulja, N., 1987: Prilog fitogeografiji Svilaje i njezina povezanost s Biokovom. Acta Biokovica, 4, 189-203.

Lovrić, A. Ž., Rac, M. \& SEkulić, B., 1989: Phytogeography and synecology of the Svilaja mountain range in Dalmatia. Period. Biol., 91(1), 177-178.

Markgraf, F., 1972: Vincetoxicum N.M. Wolf. In: Tutin, T.G., Heywood, V.H., Burges, N.A., Moore, D.M., Valentine, D.H., Walters, S.M. \& Webb, D.A., (eds.): Flora Europaea 5. University Press, Cambridge, 71-73.

Marković, LJ., 1964: Fitocenološka istraživanja ruderalne vegetacije u Hrvatskoj. Doktorska disertacija, Botanički zavod PMF-a Sveučilišta u Zagrebu, Zagreb.

Marković, LJ., 1992: Die Vegetation des Verbandes Alliarion in Kroatien. Acta Bot. Croat., 51(1), 77-88.

Marković-Gospodarić, LJ., 1965: Prilog poznavanju ruderalne vegetacije kontinentalnih krajeva Hrvatske. Acta Bot. Croat., 24, 91-136.

Marković, Lj., Iljjanić, Lj., Lukač, G. \& HršAK, V., 1993: Kvalitativni sastav flore papratnjača i sjemenjača Nacionalnog parka Krka. Botanički zavod Prirodoslovno-matematičkog fakulteta Sveučilišta u Zagrebu. Zagreb.

Mesarić, M. (ed.), 2016: Studija o utjecaju zahvata na okoliš vjetroelektrana Svilaja. JURA ENERGIJA d.o.o. za proizvodnju i distribuciju električne energije, Zagreb, 1-360.

MeŠtrović, Š., 1972: Uspijevanje primorskog bora Pinus pinaster Ait. u kulturama Hrvatske. Šumar. list, 96(5-6), 179-216.

Milović, M., 2002: The flora of Šibenik and its surroundings. Nat. Croat., 11(2), 171-223.

Milović, M., 2015: Seseli elatum L. ssp. gouanii (Koch) P. W. Ball. In: Nikolić, T., Milović, M., BogDANOvić S. \& Jasprica, N., Endemi u hrvatskoj flori, Alfa d.d., Zagreb, 419-420.

Milović, M. \& KaraĐole, J., 2016: Novi nalazi stenoendemične vrste Arenaria orbicularis Vis. u Hrvatskoj. Glas. Hrvat. bot. druš., 4(2), 25-29.

Milović, M., PAndžA, M., Jasprica, N. \& TAFra, D., 2013: Floristička istraživanja na planini Svilaji (1508 m), Dalmacija. In: Alegro, A. \& I. Boršıć (ur.), Zbornik sažetaka Četvrtog Hrvatskog Botaničkog Simpozija s međunarodnim sudjelovanjem. Hrvatsko botaničko društvo, Split, 118-119.

Milović, M., Jasprica, N., Tafra, D., PandžA, M. \& Krpina, V., 2020: Prirodna obilježja Svilaje s pregledom istraživanja flore i vegetacije. Glas. Hrvat. bot. druš., 8(1), 29-50.

Mucina, L., Bültmann, H., Dierssen, K., Teurillat, J. P., Raus, T., Čarni, A., Šumberová, K., Raus, T., Di Pietro, R., Gavílan García, R., Chytrý, M., Iakushenko, D., Schaminée, J. H. J., Bergmeier, E., Santos Guerra, A., Daniëls, F. J. A., Ermakov, N., Valachovic, M., Pignatti, S., Rodwell, J. S., Pallas, J., Capelo, J., Weber, H. E., Lysenko, T., Solomesh, A., Dimopolous, P., Aguiar, C., Freitag, H., Hennekens, S. M. \& Tichý, L., 2016: Vegetation of Europe: hierarchical floristic classification system of vascular plant, bryophyte, lichen, and algal communities. Applied Vegetation Science, 19 (Suppl. 1), 3-264.

Nıkolıć, T., 2006: Flora - priručnik za inventarizaciju i praćenje stanja. Državni zavod za zaštitu prirode. Zagreb.

Nikolić, T., 2019: Flora Croatica 4. Vaskularna flora Republike Hrvatske. Ekskurzijska flora. Alfa d.d., Zagreb.

Nikolić, T. (ed.), 2020a: Flora Hrvatske baza podataka/ Flora Croatica Database (URL: http://hirc.botanic.hr/fcd/). Prirodoslovno-matematički fakultet, Sveučilište u Zagrebu (accessed August 10, 2020).

Nikolić, T. (ed.), 2020b: Crvena knjiga vaskularne flore Hrvatske / Red Data Book of Vascular Flora of Croatia (URL: http:/ / hirc.botanic.hr/fcd/CrvenaKnjiga), Botanički zavod s Botaničkim vrtom, Prirodoslovno-matematički fakultet, Sveučilište u Zagrebu (accessed August 10, 2020). 
Nikolić, T. (ed.), 2020c: Flora Hrvatske - Alohtone biljke (URL: http://hirc.botanic.hr/fcd/ InvazivneVrste/), Botanički zavod s Botaničkim vrtom, Prirodoslovno-matematički fakultet, Sveučilište u Zagrebu (accessed August 10, 2020).

Nikolić, T. \& Topić, J. (eds.), 2005: Crvena knjiga vaskularne flore Hrvatske, Ministarstvo kulture, Državni zavod za zaštitu prirode, Republika Hrvatska, Zagreb.

Nikolić, T., Bukovec, D., Šopf, J. \& Jelaska, S.D., 1998: Kartiranje flore Hrvatske - mogućnosti i standardi (The mapping of Croatian flora - prospects and standards), Nat. Croat., 7 (Suppl. 1), 1-62.

Nikolić, T., Milović, M., Bogdanović S. \& Jasprica, N. (eds.), 2015: Endemi u hrvatskoj flori, Alfa d.d., Zagreb.

Nikolić, T., Mitić, B. \& Boršıć, I., 2014: Flora Hrvatske - invazivne biljke, Alfa d.d., Zagreb.

Nikolić, T., Jasprica, N., Bogdanović, S., Buzjak, S., Frajman, B., 2014: Nove svojte i nomenklaturne preinake u popisu flore Hrvatske. Glas. Hrvat. bot. druš., 2(1), 12-16.

PAndžA, M., 2010: The Flora of the Papuk Nature Park (Slavonia, Croatia). Šumarski list, 134 (1-2), 25-43.

Papeš, J., Marinković, R., Raić, V., Magaš N. \& Sikirica, V., 1982: Osnovna geološka karta SFRJ 1:100000, list Sinj K 33-10. Geološki zavod, Zagreb, Savezni geološki zavod, Beograd.

Petter, F., 1832: Botanischer Wegweiser in der Gegend von Spalato in Dalmatien. Battara Verlag, Zara.

Pignatti, S., 1982: Flora d'Italia, I-III. Edagricole. Bologna.

Radić, J., 1976: Bilje Biokova. Makarska, 1-237.

Raunkiaer, C., 1934: The Life Forms of Plants and Statistical Plant Geography. Oxford University Press, London.

Richardson, D. M., Pyšek, P., Rejmanek, M., Barbour, M. G., Panetta, F. D. \& West, C. J., 2000: Naturalization and invasion of alien plants: concepts and definitions. Divers. Distrib., 6, 93-107.

Rivas-Martínez, S., 1982: Etages bioclimatiques, secteur chorogiques et sésies de végétation de 1’Europe méditerranéenne. Ecol. Mediterr., 8(1-2), 275-288.

Rivas-Martínez, S., Penas, A. \& Díaz, T.E., 2004: Bioclimatic Map of Europe. Bioclimates. Cartographic Service. University of León, Spain.

RuščIĆ, M., 2002: Urbana flora Splita. Magistarski rad. Prirodoslovno-matematićki fakultet, Sveučilište u Zagrebu, 1-148.

Stevanović, V., 1996: Analysis of the Central European and Mediterranean orophytic element on the mountains of the W. and Central Balkan Peninsula, with special reference to endemies. Bocconea, 5, 77-97.

SträßSER, M., 1998; Klimadiagramme zur Köppenschen Klimaklassifikation. Stuttgart, Klett Verlag.

ŠEgota, T. \& FilıPČIĆ, A., 2003: Köppenova podjela klima i hrvatsko nazivlje, Geoadria, 8(1), 17-37.

ŠEgota, V., 2016: Proljetna flora južnih padina Svilaje. 5. Hrvatski botanički simpozij s međunarodnim sudjelovanjem, Primošten, 22.-25. rujna 2016. Knjiga sažetaka, RešETnıK, I. \& Ljubešıć, Z. (eds.), 20.

Šegota V., Jasprica N., Bogdanović S., Pandža M., Milović M. \& Alegro A. L., 2016: Distribution of the species Matthiola fruticulosa (L.) Maire (Brassicaceae) in Croatia. Glas. Hrvat. bot. druš., 4(2), 20-25.

ŠILIĆ, Č., 1984: Endemične biljke. "Svjetlost", OOUR Zavod za izdavanje udžbenika i nastavna sredstva, Sarajevo - Zavod za udžbenike, Sarajevo-Beograd, 1-227.

Šilić, Č. \& Šolić, M. E., 2002: Addition to the vascular flora in the region of Biokovo (Dalmatia, Croatia). Nat. Croat., 11(3), 341-363.

Šnvorc, Ž., Jasprica, N., Alegro, A., Kovačić, S., Franjıć, J., Krstonošić, D., Vraneša, A. \& Čarni, A., 2017: Vegetation of Croatia: Phytosociological classification of the high-rank syntaxa. Acta Bot. Croat., 76(2), 200-224.

Tafra, D., PandžA, M. \& Milović, M., 2012: Vaskularna flora grada Omiša. Nat. Croat., 21(2), 301334.

Topić, J., Iljuanić, Lj. \& Š́gulja, N., 1998: Erodium acaule (L.) Becherer (Geraniaceae), a new species in Croatian flora. Nat. Croat., 7(4), 359-362.

Trinajstić, I., 1992: Sintaksonomska analiza pašnjačke zajednice Festuco-Koelerietum H-ić 1963. Acta Bot. Croat., 51(1), 103-112. 
Tutin, T.G., Burges, N.A., Chater, A.O., Edmondson, J.R., Heywood, V.H., Moore, D.M., Valentine, D.H., Walters, S.M. \& Webb, D.A. (eds.), 1993: Flora Europaea 1, 2nd edn. University Press, Cambridge.

Tutin, T.G., Heywood, V.H., Burges, N.A., Moore, D.M., Valentine, D.H., Walters, S.M. \& Webb, D.A. (eds.), 1968-1980: Flora Europaea 2-5. University Press, Cambridge.

Verloove, F., 2006: Catalogue of neophytes in Belgium (1800-2005). Scr. Bot. Belgica, 39, 1-89.

Visiani, R., 1842: Flora Dalmatica I, Lipsiae.

Visiani, R., 1847: Flora Dalmatica II, Lipsiae.

Visiani, R., 1852: Flora Dalmatica III, Lipsiae.

Visiani, R., 1872: Florae Dalmaticae supplementum. Memor. Del R. Istituto, XVI(I), Venetiis.

Vitasović-Kosić, I., Vukojević, M. \& Bogdanović, S., 2020: First inventory of vascular flora of Matokit Mountain (Biokovo Massif, Croatia). Šumar. list, 144(5-6), 257-268.

Vladović, D., 1994: Flora Planine Mosor. Magistarski rad. Prirodoslovno-matematički fakultet, Sveučilište u Zagrebu, 1-123.

Vladović, D. \& Iljjanić, Lj., 1992: Prilog flori planine Mosor (Hrvatska). Acta Bot. Croat., 51(1), $143-150$.

Vladović, D. \& Iljuanić, LJ., 1993: Drugi prilog flori planine Mosor. Acta Bot. Croat., 52(1), 145-152.

Vladović, D., RaĐA, T. \& ŽEvrnja, N., 2012: Vaskularna flora i vegetacija jama na području srednje Dalmacije, Hrvatska. In: Čovjek i krš 2011. Zbornik radova Znanstveno-stručnog skupa Čovjek i krš 13. - 16. 10. 2011. Bijakovići - Međugorje (Lučić, I. \& J. Mulaomerović, eds.), 241-250.

Vladović, D., Mekinić, S., Piasevoli, G., Hruševar, D., Ževrnja, N., Šimić, L. \& Damjanović, T., 2019 : Značajni krajobraz Sutina. Javna ustanova za upravljanje zaštićenim dijelovima prirode na području Splitsko-dalmatinske županije - "More i krš” i Prirodoslovni muzej i zoološki vrt u Splitu, Split, 1-56.

Vukadinović, V., 2019: Interaktivna pedološka karta RH s legendom (prema Bogunović, M., VidAčEK, Ž., Racz, Z., Husnjak, S. \& Sraka, M., 1996: Namjenska pedološka karta Republike Hrvatske mjerila 1:300.000 s tumačem karata. Sveučilište u Zagrebu, Agronomski fakultet, Zavod za pedologiju, URL: http:/ / tlo-i-biljka.eu/GIS.html (accessed August 10, 2020).

Walters, S. M., Brady, A., Brickell, C. D., Cullen, J., Green, P.S., Lewis, J., Matthews, V.A., Webb, D.A., Yeo, P.F. \& Alexander, J.C.M. (eds.), 1986 - 2000: The European garden flora: A manual for the identification of plants cultivated in Europe, both out-of-doors and under glass. Cambridge University Press, Cambridge, New York, New Rochelle, Melbourne, Sydney.

Ževrnja, N. \& Vladović, D., 2005: The genus Crocus L. in the flora of Svilaja mountain. Nat. Croat., 14(4), 363-368.

\section{APPENDIX 1}

Tab. 1. The vascular plant taxa of Mt Svilaja (abbreviations are explained in the section Materials and Methods).

\begin{tabular}{|c|c|c|c|c|c|c|}
\hline Taxon & Family & \begin{tabular}{|c|} 
Life \\
form
\end{tabular} & Geoelement & Status & Localities (finding sites) & $\begin{array}{l}\text { Previously recorded } \\
\text { findings }\end{array}$ \\
\hline $\begin{array}{l}\text { Acanthus balcanicus Heywood et } \\
\text { I. Richardson }\end{array}$ & Acanthaceae & $\mathrm{H}$ & ILBA-end & & $2,3,21,23$ & $\begin{array}{l}\text { Vi-1847:226 as } A \text {. } \\
\text { longifolius Host }\end{array}$ \\
\hline Acer campestre L. & Aceraceae & $\mathrm{P}$ & EURO & & $\begin{array}{c}2,3,4,5,13,14,15,16,17,19,20,22 \\
25,28,29,37,40,42,43,44,47,55,58 \\
60,61,63,65,69,70\end{array}$ & $\begin{array}{l}\text { Je-1952; L\&al-1987, kao } \\
\text { A. c. ssp. mariscum } \\
\text { (Guss.) Hay.; V\&al-2019 }\end{array}$ \\
\hline Acer monspessulanum L. & Aceraceae & $\mathrm{P}$ & SEU-me & & $\begin{array}{c}1,6,9,13,15,20,23,25,26,27,30,40 \\
41,46,48,49,50,59,65\end{array}$ & $\begin{array}{l}\text { Ja-1908; L\&al-1987; } \\
\text { V\&al-2019 }\end{array}$ \\
\hline Acer negundo L. & Aceraceae & $\mathrm{P}$ & CUAD & inv & 14 & \\
\hline Acer obtusatum Willd. & Aceraceae & $\mathrm{P}$ & ILBA-end & & $\begin{array}{c}14,21,32,35,36,37,40,41,42,55,59 \\
61,66,69\end{array}$ & $\begin{array}{l}\text { Ja-1908; L\&al-1987; } \\
\text { VRZ-2012; Mi\&al-2013; } \\
\text { V\&al-2019 }\end{array}$ \\
\hline Acer platanoides $\mathrm{L}$ & Aceraceae & $\mathrm{P}$ & EURO & & 32 & \\
\hline Acer pseudoplatanus L. & Aceraceae & $\mathrm{P}$ & EURO & & $14,31,32,40,53,55,65$ & \\
\hline
\end{tabular}




\begin{tabular}{|c|c|c|c|c|c|c|}
\hline Taxon & Family & $\begin{array}{c}\text { Life } \\
\text { form }\end{array}$ & Geoelement & Status & Localities (finding sites) & $\begin{array}{c}\text { Previously recorded } \\
\text { findings }\end{array}$ \\
\hline Achillea clavennae L. & Asteraceae & $\mathrm{H}$ & ME-ilape & NT & Svilaja mountain range & L\&R-1989 \\
\hline Achillea collina Becker ex Rchb. & Asteraceae & $\mathrm{H}$ & CEU & & $11,16,18,19,28,43,59$ & V\&al-2019 \\
\hline Achillea millefolium $\mathrm{L}$ & Asteraceae & $\mathrm{H}$ & WISP & & 14 & \\
\hline Achillea nobilis L. & Asteraceae & $\mathrm{H}$ & EUAS & & $2,3,21,23,25,26,27,47,49,50,58,59$ & LRS-1989 \\
\hline Achillea setacea Waldst. et Kit. & Asteraceae & $\mathrm{H}$ & SEU-me & & $9,32,53$ & \\
\hline $\begin{array}{l}\text { Achnatherum calamagrostis (L.) P. } \\
\text { Beauv. }\end{array}$ & Poaceae & $\mathrm{H}$ & SEU-mo & $\begin{array}{c}\mathrm{DD}, \\
\mathrm{sp}\end{array}$ & 41,66 & \\
\hline Acinos alpinus (L.) Moench & Lamiaceae & $\mathrm{Ch}$ & SEU-mo & & 10,32 & L\&R-1989 \\
\hline Acinos arvensis (Lam.) Dandy & Lamiaceae & $\mathrm{T}$ & EURO & & \begin{tabular}{|c|}
$1,3,5,6,8,9,11,13,15,17,19,21,23$ \\
$25,26,27,31,36,38,40,43,45,47,48$ \\
$49,53,54,58,59,61,62,66,68$
\end{tabular} & VRZ-2012; V\&al-2019 \\
\hline Adiantum capillus-veneris $\mathrm{L}$. & Adiantaceae & $\mathrm{H}$ & ME-circ & NT & Significant Landscape of Sutina & V\&al-2019 \\
\hline Aegilops cylindrica Host & Poaceae & $\mathrm{T}$ & EEUPO & NT & Significant Landscape of Sutina & V\&al-2019 \\
\hline Aegilops geniculata Roth & Poaceae & $\mathrm{T}$ & ME-circ & & $1,5,17,21,25,43,45$ & V\&al-2019 \\
\hline Aegilops neglecta Req. ex Bertol. & Poaceae & $\mathrm{T}$ & ME-circ & NT & $5,20,21,23,25,43,45,46$ & \\
\hline Aegilops triuncialis L. & Poaceae & $\mathrm{T}$ & ME-circ & & $1,5,25,45$ & V\&al-2019 \\
\hline Aesculus hippocastanum L. & $\begin{array}{c}\text { Hippo- } \\
\text { castanaceae }\end{array}$ & $P$ & CUAD & & $14,22^{*}, 46^{*}$ & \\
\hline $\begin{array}{l}\text { Aethionema saxatile (L.) R.Br. ssp. } \\
\text { saxatile }\end{array}$ & Brassicaceae & $\mathrm{Ch}$ & SEU-me & & $\begin{array}{c}9,11,17,18,23,26,27,31,33,37,41 \\
43,45,48,49,51,52,54,56,58,61,64 \\
69\end{array}$ & $\begin{array}{l}\text { VRZ-2012, V\&al-2019 as } \\
\text { A. saxatile (L.) R.Br. }\end{array}$ \\
\hline $\begin{array}{l}\text { Agrimonia eupatoria L. ssp. } \\
\text { eupatoria }\end{array}$ & Rosaceae & $\mathrm{H}$ & $\mathrm{CIHO}$ & & $\begin{array}{c}1,4,9,11,13,15,17,18,19,21,22,23 \\
25,28,30,31,37,38,40,43,44,45,46 \\
47,48,50,51,53,58,59,60,62,65,66 \\
67,69,70\end{array}$ & $\begin{array}{l}\text { V\&al-2019 as } A \text {. } \\
\text { eupatoria } L .\end{array}$ \\
\hline Agrostemma githago L. & Caryophyllaceae & $\mathrm{T}$ & WISP & & 3,67 & \\
\hline Agrostis capillaris L. & Poaceae & $\mathrm{H}$ & $\mathrm{CIHO}$ & & $9,11,59$ & V\&al-2019 \\
\hline Agrostis castellana Boiss. et Reut. & Poaceae & $\mathrm{H}$ & ME-atl & DD & 22 & \\
\hline Agrostis stolonifera L. & Poaceae & $\mathrm{H}$ & $\mathrm{CIHO}$ & & Significant Landscape of Sutina & L\&R-1989; V\&al-2019 \\
\hline Ailanthus altissima (Mill.) Swingle & Simaroubaceae & P & CUAD & inv & $\begin{array}{c}1,2,3,4,5,13,14,15,17,21,23,25 \\
29,30,32,37,42,43,45,47,69,70\end{array}$ & V\&al-2019 \\
\hline Aira elegantissima Schur & Poaceae & $\mathrm{T}$ & SEU-me & & 8,55 & \\
\hline Ajuga chamaepitys (L.) Schreb. & Lamiaceae & $\mathrm{T}$ & ME-circ & & $21,24,68,69$ & \\
\hline Ajuga genevensis L. & Lamiaceae & $\mathrm{H}$ & EUAS & & $\begin{array}{c}2,11,20,29,30,37,42,44,47,60,63 \\
65,70\end{array}$ & V\&al-2019 \\
\hline Ajuga reptans $\mathrm{L}$. & Lamiaceae & $\mathrm{H}$ & EUAS & & $38,65,66^{*}$ & \\
\hline Alcea rosea $\mathrm{L}$. & Malvaceae & $\mathrm{H}$ & CUAD & & $1,4,5,23,43,46$ & \\
\hline Alisma plantago-aquatica L. & Alismataceae & $\mathrm{Hy}$ & WISP & & $22,28,46$ & \\
\hline $\begin{array}{l}\text { Alliaria petiolata (M. Bieb.) Cavara } \\
\text { et Grande }\end{array}$ & Brassicaceae & $\mathrm{H}$ & EUAS & & $14,20,27,29,30,69$ & Ma-1992; V\&al-2019 \\
\hline Allium amethystinum Tausch & Amaryllidaceae & G & ME-east & & 23,43 & \\
\hline Allium ampeloprasum $\mathrm{L}$. & Amaryllidaceae & G & ME-circ & & 3,5 & \\
\hline Allium carinatum $\mathrm{L}$. & Amaryllidaceae & G & EURO & & 22 & V\&al-2019 \\
\hline Allium cepa $\mathrm{L}$. & Amaryllidaceae & G & CUAD & & $\begin{array}{c}1,13^{*}, 14^{*}, 16^{*}, 25^{*}, 27,28^{*}, 37^{*}, 43^{*}, \\
47^{*}, 66^{*}, 69\end{array}$ & \\
\hline Allium flavum $\mathrm{L}$. & Amaryllidaceae & G & SEU-me & & $1,15,31$ & V\&al-2019 \\
\hline $\begin{array}{l}\text { Allium guttatum Steven ssp. dal- } \\
\text { maticum (A. Kern.ex Janch.) Stearn }\end{array}$ & Amaryllidaceae & G & ILBA-end & & Significant Landscape of Sutina & V\&al-2019 \\
\hline Allium horvatii Lovrić & Amaryllidaceae & G & ME-ilade & $\begin{array}{l}\text { end, } \\
\text { NT, sp }\end{array}$ & 2 & $\begin{array}{l}\text { Vi-1842:133 as A. saxatile } \\
\text { Bieb.; L\&al-1987, as A. s. } \\
\text { ssp. tergestinum (M. } \\
\text { Gand.) Bed. et Lov. }\end{array}$ \\
\hline Allium lusitanicum Lam. & Amaryllidaceae & G & SEU-po & & 41 & $\begin{array}{l}\text { Vi-1842:134, as A. fallax } \\
\text { Schult. }\end{array}$ \\
\hline Allium moschatum L. & Amaryllidaceae & G & SEEU & & 31 & \\
\hline Allium oleraceum L. & Amaryllidaceae & G & EUAS & & $14,15,12,28,43,70$ & \\
\hline $\begin{array}{l}\text { Allium paniculatum L. ssp. fuscum } \\
\text { (Waldst.et Kit.) Arcang. }\end{array}$ & Amaryllidaceae & G & SEU-me & & $1,15,23,24,25,31,43$ & \\
\hline $\begin{array}{l}\text { Allium sphaerocephalon L. ssp. } \\
\text { sphaerocephalon }\end{array}$ & Amaryllidaceae & G & SEU-me & & $\begin{array}{c}1,8,11,23,24,32,33,34,35,38,40 \\
41,43,51,53,56,58,59,62,65\end{array}$ & $\begin{array}{l}\text { V\&al-2019, as } A \text {. } \\
\text { sphaerocephalon L. }\end{array}$ \\
\hline Allium vineale $\mathrm{L}$. & Amaryllidaceae & G & WISP & LC & Significant Landscape of Sutina & V\&al-2019 \\
\hline
\end{tabular}




\begin{tabular}{|c|c|c|c|c|c|c|}
\hline Taxon & Family & \begin{tabular}{|c|} 
Life \\
form
\end{tabular} & Geoelement & Status & Localities (finding sites) & $\begin{array}{l}\text { Previously recorded } \\
\text { findings }\end{array}$ \\
\hline Alopecurus myosuroides Huds. & Poaceae & $\mathrm{T}$ & WISP & & 37 & \\
\hline Alopecurus rendlei Eig. & Poaceae & $\mathrm{T}$ & SEU-me & $\begin{array}{l}\text { VU, } \\
\text { sp }\end{array}$ & 28 & \\
\hline Althaea cannabina $\mathrm{L}$. & Malvaceae & $\mathrm{H}$ & SEU-po & & $1,5,16,20,29,30$ & \\
\hline Althaea hirsuta L. & Malvaceae & $\mathrm{T}$ & SEU-me & & 21,23 & \\
\hline Alyssum alyssoides (L.) L & Brassicaceae & $\mathrm{T}$ & SEU-me & & $\begin{array}{c}2,4,6,8,9,11,17,20,27,30,42,44 \\
45,55,58,59,70\end{array}$ & V\&al-2019 \\
\hline $\begin{array}{l}\text { Alyssum montanum L. ssp. } \\
\text { montanum }\end{array}$ & Brassicaceae & $\mathrm{Ch}$ & SEU-me & & $44,49,51,65,67$ & $\begin{array}{l}\text { V\&al- } 2019 \text { as } A \text {. } \\
\text { montanum } \mathrm{L} \text {. }\end{array}$ \\
\hline Alyssum simplex Rudolphi & Brassicaceae & $\mathrm{T}$ & ME-circ & & $1,2,5,21,25,29,43,45$ & V\&al-2019 \\
\hline Amaranthus albus L. & Amaranthaceae & $\mathrm{T}$ & WISP & inv & $4,22,68$ & \\
\hline Amaranthus blitoides S. Watson & Amaranthaceae & $\mathrm{T}$ & CUAD & inv & 5,68 & \\
\hline $\begin{array}{l}\text { Amaranthus cruentus (Lesp. et } \\
\text { Thév.) N.Terracc. }\end{array}$ & Amaranthaceae & $\mathrm{T}$ & CUAD & & 50 & \\
\hline Amaranthus deflexus L. & Amaranthaceae & $\mathrm{T}$ & WISP & inv & $1,3,5,14,27$ & \\
\hline Amaranthus graecizans $\mathrm{L}$. & Amaranthaceae & $\mathrm{T}$ & WISP & & $4,14,27$ & \\
\hline Amaranthus hybridus L. & Amaranthaceae & $\mathrm{T}$ & WISP & inv & 4 & \\
\hline Amaranthus powellii S. Watson & Amaranthaceae & $\mathrm{T}$ & CUAD & & $13,27,66$ & \\
\hline Amaranthus retroflexus L. & Amaranthaceae & $\mathrm{T}$ & WISP & inv & $3,4,13,14,22,24,27,65,66,68$ & V\&al-2019 \\
\hline Ambrosia atemisiifolia $\mathrm{L}$. & Asteraceae & $\mathrm{T}$ & CUAD & inv & $4,13,22,68$ & \\
\hline Amelanchier ovalis Medik. & Rosaceae & $\mathrm{P}$ & SEU-po & & $\begin{array}{c}9,11,33,34,36,38,39,40,41,51,52 \\
64,65,67\end{array}$ & $\begin{array}{l}\text { Ja-1908; L\&al-1987 as A. } \\
\text { cretica Pers.; V\&al-2019 }\end{array}$ \\
\hline Amorpha fruticosa $\mathrm{L}$. & Fabaceae & $\mathrm{P}$ & CUAD & inv & 28 & \\
\hline Anacamptis pyramidalis (L.) Rich. & Orchidaceae & G & EURO & NT, sp & $13,39,41,42,45,46,47,51,53,58$ & $\begin{array}{l}\text { Kr-2005:43; Df-2006, } \\
\text { V\&al-2019 }\end{array}$ \\
\hline Anagallis arvensis L. & Primulaceae & $\mathrm{T}$ & WISP & & $3,9,11,14,26,27,37,45,46,65$ & V\&al-2019 \\
\hline Anagallis coerulea Schreb. & Primulaceae & $\mathrm{T}$ & WISP & & 14,28 & V\&al-2019 \\
\hline Anchusa italica Retz. & Boraginaceae & $\mathrm{H}$ & SEU-me & & 3,24 & \\
\hline $\begin{array}{l}\text { Anchusella cretica (Mill.) Bigazzi, } \\
\text { E. Nardi et Salvi }\end{array}$ & Boraginaceae & $\mathrm{T}$ & ME-east & & $2,27,29,30$ & \\
\hline Anemone nemorosa L. & Ranunculaceae & G & $\mathrm{CIHO}$ & & $10,12,36,67$ & $\begin{array}{l}\text { Vi-1852:79; Mi\&al-2013; } \\
\text { V\&al-2019 }\end{array}$ \\
\hline Anemone ranunculoides $\mathrm{L}$. & Ranunculaceae & G & EUAS & & $10,11,32$ & Vi-1852:80 \\
\hline Angelica sylvestris L. & Apiaceae & $\mathrm{H}$ & EUAS & & Significant Landscape of Sutina & V\&al-2019 \\
\hline Anthemis arvensis L. & Asteraceae & $\mathrm{T}$ & ME-circ & & $4,5,13,20,21,23,24,25,26,27,66$ & \\
\hline Anthemis cotula $\mathrm{L}$. & Asteraceae & Ch & WISP & & Significant Landscape of Sutina & V\&al-2019 \\
\hline Anthemis segetalis Ten. & Asteraceae & $\mathrm{T}$ & ME-ilseu & & $4,11,20,22,23,27$ & V\&al-2019 \\
\hline Anthemis tinctoria $\mathrm{L}$. & Asteraceae & $\mathrm{H}$ & SEU-po & & 59 & \\
\hline Anthericum liliago L. & Asparagaceae & G & SEU-me & & 34 & V\&al-2019 \\
\hline Anthericum ramosum $\mathrm{L}$. & Asparagaceae & G & CEU & & $9,26,31,33,34,39,41,56$ & V\&al-2019 \\
\hline Anthoxanthum odoratum $\mathrm{L}$. & Poaceae & $\mathrm{H}$ & EUAS & & $\begin{array}{c}2,8,11,14,16,25,26,28,37,40,42 \\
43,44,46,47,53,55,57,60,61,65,67 \\
69,70\end{array}$ & V\&al-2019 \\
\hline Anthriscus cerefolium (L.) Hoffm. & Apiaceae & $\mathrm{T}$ & CUAD & & Significant Landscape of Sutina & V\&al-2019 \\
\hline Anthyllis aurea Host & Fabaceae & $\mathrm{Ch}$ & ILBA-end & & Svilaja mountain range & LRS-1989 \\
\hline $\begin{array}{l}\text { Anthyllis montana L. ssp. } \\
\text { atropurpurea (Vuk.) Pignatti }\end{array}$ & Fabaceae & $\mathrm{H}$ & ILBA-bap & $\begin{array}{l}\text { end, } \\
\text { sp }\end{array}$ & Svilaja mountain range & LRS-1989 \\
\hline $\begin{array}{l}\text { Anthyllis montana L. ssp. jacquinii } \\
\text { (A. Kern.) Hayek }\end{array}$ & Fabaceae & $\mathrm{H}$ & ILBA-end & $\mathrm{LC}$ & $8,9,10,33,34$ & \\
\hline Anthyllis vulneraria $\mathrm{L}$. & Fabaceae & $\mathrm{H}$ & ME-eu & & $33,34,39,40,52,53,55,56,57,58,62$ & $\begin{array}{l}\text { Pe-1832 and Tr-1992, as } \\
\text { A. rubicunda Wender. }\end{array}$ \\
\hline $\begin{array}{l}\text { Anthyllis vulneraria L. ssp. } \\
\text { praepropera (A.Kern.) Bornm (= } \\
\text { A. rubiflora) }\end{array}$ & Fabaceae & $\mathrm{H}$ & ME-eu & & $8,9,11,26,31,53,59,65,67$ & Se\&al-2016; V\&al-2019 \\
\hline $\begin{array}{l}\text { Anthyllis vulneraria L. ssp. } \\
\text { weldeniana (Rchb.) Cullen }\end{array}$ & Fabaceae & $\mathrm{H}$ & ME-eu & $\begin{array}{l}\text { end, } \\
\text { sp }\end{array}$ & $17,23,25,29,30,32,42,45$ & \\
\hline Antirrhinum majus L. & Scrophulariaceae & $\mathrm{Ch}$ & ME-west & & $1,14,23$ & M\&K-2016 \\
\hline Arabis collina Ten. & Brassicaceae & $\mathrm{H}$ & SEU-me & & $9,10,12,30,32,33,36,37,51,64,70$ & $\begin{array}{l}\text { Vi-1847:127 as A. muralis } \\
\text { Bertol. }\end{array}$ \\
\hline Arabis glabra (L.) Bernhardt & Brassicaceae & $\mathrm{H}$ & WISP & & $29,44,69$ & \\
\hline
\end{tabular}




\begin{tabular}{|c|c|c|c|c|c|c|}
\hline Taxon & Family & $\begin{array}{c}\text { Life } \\
\text { form }\end{array}$ & Geoelement & Status & Localities (finding sites) & $\begin{array}{c}\text { Previously recorded } \\
\text { findings }\end{array}$ \\
\hline Arabis hirsuta (L.) Scop. & Brassicaceae & $\mathrm{H}$ & WISP & & $\begin{array}{c}1,6,8,9,11,13,16,17,18,19,22,26 \\
29,31,38,39,40,42,44,45,46,47,50 \\
52,53,55,56\end{array}$ & V\&al-2019 \\
\hline Arabis planisiliqua (Pers.) Rchb. & Brassicaceae & $\mathrm{H}$ & ME-west & & 17 & \\
\hline Arabis sagittata (Bertol.) DC. & Brassicaceae & $\mathrm{H}$ & EUAS & & 35 & \\
\hline Arabis scopoliana Boiss. & Brassicaceae & $\mathrm{Ch}$ & ME-ilade & $\begin{array}{c}\text { end, } \\
\text { sp }\end{array}$ & 33,34 & Ja-1908; L\&R-1989 \\
\hline Arabis turrita $\mathrm{L}$. & Brassicaceae & $\mathrm{H}$ & SEU-me & & $\begin{array}{c}10,11,12,30,31,32,34,36,37,40,44 \\
50,57,69\end{array}$ & V\&al-2019 \\
\hline $\begin{array}{l}\text { Arceuthobium oxycedri (DC.) M. } \\
\text { Bieb. }\end{array}$ & Loranthaceae & $P$ & SEU-po & & 62,64 & \\
\hline Arctium lappa $\mathrm{L}$. & Asteraceae & $\mathrm{H}$ & EUAS & & $13,20,66$ & V\&al-2019 \\
\hline Arctium minus Bernh. & Asteraceae & $\mathrm{H}$ & EUAS & & $1,3,4,14,16,17,32,46,61,69 ?, 70$ & \\
\hline $\begin{array}{l}\text { Arctostaphylos uva-ursi (L.) } \\
\text { Spreng. }\end{array}$ & Ericaceae & $\mathrm{Ch}$ & $\mathrm{CIHO}$ & $\begin{array}{l}\text { VU, } \\
\mathrm{sp}\end{array}$ & 11 & \\
\hline $\begin{array}{l}\text { Aremonia agrimonoides (L.) DC. } \\
\text { ssp. agrimonioides }\end{array}$ & Rosaceae & $\mathrm{H}$ & $\mathrm{CIHO}$ & & 35,36 & Je-1952 \\
\hline $\begin{array}{l}\text { Arenaria leptoclados (Reichenb.) } \\
\text { Guss }\end{array}$ & Caryophyllaceae & $\mathrm{T}$ & EUAS & & $\begin{array}{c}1,2,3,11,14,16,17,20,22,25,27,36 \\
38,42,45,49,57,66\end{array}$ & M\&K-2016 \\
\hline Arenaria orbicularis Vis. & Caryophyllaceae & $\mathrm{Ch}$ & ME-ilade & $\begin{array}{c}\text { end, } \\
\text { DD, } \\
\text { sp }\end{array}$ & 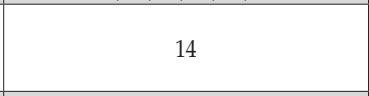 & M\&K-2016 \\
\hline Arenaria serpillyfolia $\mathrm{L}$. & Caryophyllaceae & $\mathrm{T}$ & WISP & & $6,8,11,29,36,37,44,45,55,58,69$ & V\&al-2019 \\
\hline $\begin{array}{l}\text { Argyrolobium zanonii (Turra) P. } \\
\text { W. Ball }\end{array}$ & Fabaceae & $\mathrm{Ch}$ & ME-west & & $25,26,45,47,61$ & \\
\hline Aristolochia clematitis L. & Aristolochiaceae & G & SEU-po & & $3,22,37,69$ & \\
\hline Aristolochia lutea Desf. & Aristolochiaceae & G & SEEU & & $\begin{array}{c}2,11,13,14,18,20,29,34,42,49,65 \\
67\end{array}$ & $\begin{array}{l}\text { L\&al-1987, } \\
\text { as A. lutea Desf. } \\
\text { (A. pallida var. istriaca } \\
\text { Posp.) }\end{array}$ \\
\hline Aristolochia rotunda L. & Aristolochiaceae & G & ME-circ & & $16,21,22,23,28,37,42,60,70$ & \\
\hline $\begin{array}{l}\text { Armeria canescens (Host) Boiss. in } \\
\text { DC. ssp. canescens }\end{array}$ & Plumbaginaceae & $\mathrm{H}$ & ME-east & & $7,8,11,34,48,49,54,55,66$ & $\begin{array}{l}\text { L\&al-1987, as A. } \\
\text { canescens Host }\end{array}$ \\
\hline $\begin{array}{l}\text { Armoracia rusticana P. Gaertn., B. } \\
\text { Mey. et Scherb }\end{array}$ & Brassicaceae & G & WISP & & $1,17,23,49,69$ & \\
\hline $\begin{array}{l}\text { Arrhenatherum elatius (L.) P. } \\
\text { Beauv. ex J.Presl et C.Presl }\end{array}$ & Poaceae & $\mathrm{H}$ & EURO & & $11,14,16,21,32,42,65,69$ & \\
\hline Artemisia absinthium L. & Asteraceae & $\mathrm{Ch}$ & EUAS & & $\begin{array}{c}1,3,4,6,13,14,17,22,25,32,38,39 \\
40,59,68\end{array}$ & Ma-1964; V\&al-2019 \\
\hline Artemisia alba Turra & Asteraceae & $\mathrm{Ch}$ & SEU-me & & 30,49 & \\
\hline Artemisia annua L. & Asteraceae & $\mathrm{T}$ & CUAD & inv & 6 & \\
\hline Artemisia campestris L. & Asteraceae & $\mathrm{Ch}$ & $\mathrm{CIHO}$ & & $1,2,13,15,26,29,30,31,47,48,49,54$ & V\&al-2019 \\
\hline Artemisia scoparia Waldst. et Kit. & Asteraceae & $\mathrm{H}$ & EEUPO & & 7,8 & Vi-1847:92 \\
\hline Artemisia verlotiorum Lamotte & Asteraceae & $\mathrm{H}$ & CUAD & inv & $13,14,15,36,38,51,65$ & \\
\hline Artemisia vulgaris L. & Asteraceae & $\mathrm{H}$ & WISP & & $1,14,17,32,57,59,65,66,70$ & Ma-1992; V\&al-2019 \\
\hline Arum italicum Mill. & Araceae & G & ME-circ & & $\begin{array}{c}2,4,5,14,15,18,25,27,30 ?, 37,60 \\
69,70\end{array}$ & V\&al-2019 \\
\hline Arum maculatum L. & Araceae & G & EURO & & $20,29,44,63$ & \\
\hline Asparagus acutifolius L. & Asparagaceae & G & ME-circ & & $\begin{array}{c}1,2,3,4,5,22,23,24,27,30,37,43 \\
45,47,60,61,69,70\end{array}$ & V\&al-2019 \\
\hline Asparagus officinalis L. & Asparagaceae & G & CUAD & NT & 43 & \\
\hline $\begin{array}{l}\text { Asperula aristata L.f. ssp. } \\
\text { condensata (Heldr.ex Boiss.) } \\
\text { Ehrend.et Krendl }\end{array}$ & Rubiaceae & $\mathrm{H}$ & ILBA-end & & 48 & \\
\hline $\begin{array}{l}\text { Asperula aristata L.f. ssp. scabra } \\
\text { (J.Presl et C.Presl) Nyman }\end{array}$ & Rubiaceae & $\mathrm{H}$ & SEU-me & & $\begin{array}{c}6,7,8,9,23,25,26,27,31,43,48,65 \\
68\end{array}$ & $\begin{array}{l}\text { Vi-1852:11 as A. } \\
\text { cynanchica y canescens; } \\
\text { LRS-1989, as A. scabra } \\
\text { Presl. (A. canescens Vis.); } \\
\text { V\&al-2019 as A. aristata } \\
\text { L.f. }\end{array}$ \\
\hline Asperula cynanchica $\mathrm{L}$. & Rubiaceae & $\mathrm{H}$ & ME-circ & & $\begin{array}{c}\text { Sutina; } \\
\text { Significant Landscape of Sutina }\end{array}$ & Je-1952; V\&al-2019 \\
\hline
\end{tabular}




\begin{tabular}{|c|c|c|c|c|c|c|}
\hline Taxon & Family & \begin{tabular}{|c|} 
Life \\
form
\end{tabular} & Geoelement & Status & Localities (finding sites) & $\begin{array}{l}\text { Previously recorded } \\
\text { findings }\end{array}$ \\
\hline Asperula purpurea (L.) Ehrend. & Rubiaceae & $\mathrm{Ch}$ & SEEU & & $\begin{array}{c}8,9,11,26,31,32,33,34,35,38,39 \\
43,51,54,56,65,66\end{array}$ & $\begin{array}{l}\text { Vi-1852:6 as Galium } \\
\text { purpureum L. }\end{array}$ \\
\hline Asperula scutellaris Vis. & Rubiaceae & $\mathrm{Ch}$ & ME-ilade & $\begin{array}{l}\text { end, } \\
\text { sp }\end{array}$ & Svilaja mountain range & LRS-1989 \\
\hline Asphodeline lutea (L.) Rchb. & Xanthorrhoeaceae & G & ME-east & & Svilaja mountain range & LRS-1989 \\
\hline Asplenium adiantum-nigrum L. & Aspleniaceae & $\mathrm{H}$ & WISP & & Significant Landscape of Sutina & V\&al-2019 \\
\hline Asplenium ceterach $\mathrm{L}$. & Aspleniaceae & $\mathrm{H}$ & SEU-me & & $\begin{array}{c}2,5,6,10,13,14,18,19,20,21,22,26 \\
27,29,30,31,36,37,40,42,44,45,46, \\
48,55,61,65\end{array}$ & $\begin{array}{l}\text { M\&K-2016; VRZ-2012; } \\
\text { V\&al-2019 }\end{array}$ \\
\hline $\begin{array}{l}\text { Asplenium ceterach L. ssp. bivalens } \\
\text { (D. E. Meyer) Greuter et Burdet }\end{array}$ & Aspleniaceae & $\mathrm{H}$ & SEEU & & hilly rocks in the east of Svilaja & $\begin{array}{l}\text { L\&al-1987, as Ceterach } \\
\text { javorkeana (Vida) Soo }\end{array}$ \\
\hline Asplenium lepidum C Presl & Aspleniaceae & $\mathrm{H}$ & SEEU & & $10,14,29,32,52$ & \\
\hline Asplenium onopteris L. & Aspleniaceae & $\mathrm{H}$ & EEUPO & & $11,14,69$ & \\
\hline Asplenium ruta-muraria L. & Aspleniaceae & $\mathrm{H}$ & $\mathrm{CIHO}$ & & $\begin{array}{c}11,14,15,18,22,31,37,40,50,52,53, \\
65\end{array}$ & $\begin{array}{l}\text { M\&K-2016; VRZ-2012; } \\
\text { V\&al-2019 }\end{array}$ \\
\hline Asplenium trichomanes L. & Aspleniaceae & $\mathrm{H}$ & WISP & & $\begin{array}{c}5,11,14,15,18,20,21,27,29,31,32 \\
35,36,37,38,40,42,55,64,65\end{array}$ & $\begin{array}{l}\text { M\&K-2016; VRZ-2012; } \\
\text { V\&al-2019 }\end{array}$ \\
\hline Aster lanceolatus Willd. & Asteraceae & $\mathrm{H}$ & CUAD & & 28,46 & \\
\hline Aster amellus L. & Asteraceae & $\mathrm{H}$ & EEUPO & & 52 & V\&al-2019 \\
\hline Aster novi-belgii $\mathrm{L}$. & Asteraceae & $\mathrm{H}$ & CUAD & & 51 & \\
\hline Astragalus glycyphyllos L & Fabaceae & $\mathrm{H}$ & EUAS & & $14,21,32,44,47,55,63$ & V\&al-2019 \\
\hline $\begin{array}{l}\text { Astragalus monspessulanus L. ssp. } \\
\text { illyricus (Bernhardt) Chater }\end{array}$ & Fabaceae & $\mathrm{H}$ & ME-ilade & $\begin{array}{l}\text { end, } \\
\text { sp }\end{array}$ & $\begin{array}{c}2,13,25,30,31,37,42,44,45,46,47 \\
57,58,60,61,62,64,69,70\end{array}$ & $\begin{array}{l}\text { Je-1952, as A. illyricus; } \\
\text { V\&al-2019 }\end{array}$ \\
\hline $\begin{array}{l}\text { Astragalus muelleri Steud. et } \\
\text { Hochst. }\end{array}$ & Fabaceae & $\mathrm{H}$ & ME-ilade & $\begin{array}{c}\text { end, } \\
\text { NT, sp }\end{array}$ & $1,69,70$ & \\
\hline $\begin{array}{l}\text { Astragalus vesicarius L. ssp. } \\
\text { carniolicus (A. Kern.) Chater }\end{array}$ & Fabaceae & $\mathrm{H}$ & SEU-po & & 33 & \\
\hline Athamanta cretensis L. & Apiaceae & $\mathrm{H}$ & SEU-mo & & 33 & L\&R-1989 \\
\hline Athyrium filix-femina (L.) Roth & Woodsiaceae & $\mathrm{H}$ & WISP & & Significant Landscape of Sutina & V\&al-2019 \\
\hline Atriplex patula L. & Chenopodiaceae & $\mathrm{T}$ & WISP & & $4,13,14,16,50$ & \\
\hline Atropa bella-donna $\mathrm{L}$. & Solanaceae & $\mathrm{H}$ & EURO & & 32,35 & V\&al-2019 \\
\hline Avena barbata Pott ex Link & Poaceae & $\mathrm{T}$ & SEU-po & & $1,3,5,23,25,28,37,43,45,70$ & V\&al-2019 \\
\hline Avena sativa $\mathrm{L}$. & Poaceae & $\mathrm{T}$ & CUAD & & 3 & \\
\hline Avena sterilis L. & Poaceae & $\mathrm{T}$ & SEU-po & & $14,23,28,43,46$ & \\
\hline $\begin{array}{l}\text { Avenula pubescens (Huds.) } \\
\text { Dumort. }\end{array}$ & Poaceae & $\mathrm{H}$ & EUAS & & Significant Landscape of Sutina & V\&al-2019 \\
\hline Ballota nigra L. ssp. foetida Hayek & Lamiaceae & $\mathrm{H}$ & SEU-me & & $\begin{array}{c}1,3,4,13,14,16,20,21,25,27,30,42 \\
43,44,49,65,68\end{array}$ & Ma-1992 \\
\hline Barbarea vulgaris R. Br. & Brassicaceae & $\mathrm{H}$ & WISP & & 3 & V\&al-2019 \\
\hline Bassia scoparia (L.) A. J. Scott & Chenopodiaceae & $\mathrm{T}$ & CUAD & & 1 & \\
\hline Bellis perennis $\mathrm{L}$. & Asteraceae & $\mathrm{H}$ & CEU & & $14,16,20,37,42,64,69,70$ & V\&al-2019 \\
\hline Berteroa mutabilis (Vent.) DC. & Brassicaceae & $\mathrm{H}$ & EUAS & & $1,3,4,6,13,14,27,48,50,55$ & V\&al-2019 \\
\hline Berula erecta (Huds.) Coville & Apiaceae & G & $\mathrm{CIHO}$ & & 14 & \\
\hline Beta vulgaris L. ssp. vulgaris & Chenopodiaceae & $\mathrm{T}$ & CUAD & LC & $\begin{array}{c}14^{*}, 16^{*}, 22^{*}, 25^{*}, 27,28,37^{*}, 43^{*}, 43, \\
49^{*}\end{array}$ & $\begin{array}{l}\text { Vi-1842:241 as B. } \\
\text { maritima L. }\end{array}$ \\
\hline $\begin{array}{l}\text { Betonica officinalis L. ssp. serotina } \\
\text { (Host) Murb. }\end{array}$ & Lamiaceae & $\mathrm{H}$ & EURO & & $\begin{array}{c}2,6,10,19,25,26,34,35,36,39,41 \\
49,51,53,65,67\end{array}$ & $\begin{array}{l}\text { V\&al-2019 as B. } \\
\text { officinalis L. }\end{array}$ \\
\hline Betula pendula Roth & Betulaceae & $\mathrm{P}$ & CUAD & & 55,65 & \\
\hline Bidens subalternans DC. & Asteraceae & $\mathrm{T}$ & CUAD & inv & 4,27 & \\
\hline Bidens tripartita $\mathrm{L}$. & Asteraceae & $\mathrm{T}$ & EUAS & & 14,64 & \\
\hline Bifora radians M. Bieb. & Apiaceae & $\mathrm{T}$ & SEU-me & & 3 & Vi-1852:72 \\
\hline $\begin{array}{l}\text { Biscutella laevigata L. ssp. lucida } \\
\text { (Balbis ex DC.) Mach.-Laur. }\end{array}$ & Brassicaceae & $\mathrm{H}$ & SEU-mo & & 32,64 & $\begin{array}{l}\text { V\&al-2019 as B. laevigata } \\
\text { L. }\end{array}$ \\
\hline Bituminaria bituminosa $($ L.) Stirton & Fabaceae & $\mathrm{H}$ & ME-circ & & $25,37,43,46,64,68,69,70$ & \\
\hline $\begin{array}{l}\text { Blackstonia perfoliata (L.) Huds. } \\
\text { ssp. perfoliata }\end{array}$ & Gentianaceae & $\mathrm{T}$ & ME-atl & & 46,47 & \\
\hline $\begin{array}{l}\text { Blysmus compressus (L.) Panz. ex } \\
\text { Link }\end{array}$ & Cyperaceae & G & EUAS & $\mathrm{EN}, \mathrm{sp}$ & Svilaja mountain range & L\&R-1989 \\
\hline Bombycilaena erecta (L.) Smoljan. & Asteraceae & $\mathrm{T}$ & SEU-me & & $1,5,8,13,18,23,25,26,27,48$ & \\
\hline $\begin{array}{l}\text { Brachypodium distachyon (L.) P. } \\
\text { Beauv. }\end{array}$ & Poaceae & $\mathrm{T}$ & ME-circ & & 23 & \\
\hline
\end{tabular}




\begin{tabular}{|c|c|c|c|c|c|c|}
\hline Taxon & Family & $\begin{array}{c}\text { Life } \\
\text { form }\end{array}$ & Geoelement & Status & Localities (finding sites) & $\begin{array}{c}\text { Previously recorded } \\
\text { findings }\end{array}$ \\
\hline $\begin{array}{l}\text { Brachypodium pinnatum (L.) P. } \\
\text { Beauv. ssp. rupestre (Host) } \\
\text { Schübl.et M. Martens }\end{array}$ & Poaceae & $\mathrm{H}$ & SEU-me & & $3,16,17,21,22,25,31,34,46$ & \\
\hline $\begin{array}{l}\text { Brachypodium pinnatum }(\mathrm{L} .) \\
\text { P.Beauv. ssp. pinnatum }\end{array}$ & Poaceae & $\mathrm{H}$ & WISP & & $\begin{array}{c}7,8,11,15,18,19,22,37,39,40,44 ? \\
50,56,61,62\end{array}$ & \\
\hline $\begin{array}{l}\text { Brachypodium sylvaticum (Huds.) } \\
\text { P. Beauv. }\end{array}$ & Poaceae & $\mathrm{H}$ & EUAS & & $4,14,16,46,63,70$ & Je-1952; V\&al-2019 \\
\hline Brassica oleracea L. & Brassicaceae & $\mathrm{Ch}$ & CUAD & & $4,14^{*}, 16^{*}, 25^{*}$ & \\
\hline Briza media $\mathrm{L}$. & Poaceae & $\mathrm{H}$ & EUAS & & 39 & \\
\hline Bromus arvensis L. & Poaceae & $\mathrm{T}$ & EUAS & & 1,69 & V\&al-2019 \\
\hline $\begin{array}{l}\text { Bromus erectus Huds. ssp. } \\
\text { condensatus (Hack.) Asch. et } \\
\text { Graebn. }\end{array}$ & Poaceae & $\mathrm{H}$ & SEU-me & & $9,11,30,33,39,49,55,56,58,62$ & \\
\hline Bromus erectus Huds. ssp. erectus & Poaceae & $\mathrm{H}$ & WISP & & $\begin{array}{c}1,5,6,8,9,11,16,17,18,19,20,23 \\
24,26,28,30,36,39,41,45,46,51,55 \\
56,59,60,62,70\end{array}$ & $\begin{array}{l}\text { Je-1952, Tr-1992, } \\
\text { L\&R-1989 and } \\
\text { V\&al-2019 as B erectus } \\
\text { Huds. (s.l.) }\end{array}$ \\
\hline $\begin{array}{l}\text { Bromus hordeaceus L. ssp. } \\
\text { molliformis (Lloyd) Maire et } \\
\text { Weiller } \\
\end{array}$ & Poaceae & $\mathrm{T}$ & SEU-me & & $\begin{array}{c}5,14,16,17,20,37,42,51,63,67,69 \\
70\end{array}$ & $\begin{array}{l}\text { V\&al-2019 as B. } \\
\text { hordaceus L. }\end{array}$ \\
\hline Bromus inermis Leyss. & Poaceae & $\mathrm{H}$ & EUAS & & 9,41 & \\
\hline Bromus japonicus Thunb. & Poaceae & $\mathrm{T}$ & EUAS & & 9,17 & \\
\hline Bromus madritensis L. & Poaceae & $\mathrm{T}$ & ME-atl & & 1,43 & \\
\hline Bromus racemosus L. & Poaceae & $\mathrm{T}$ & WISP & & 28 & \\
\hline Bromus rigidus Roth & Poaceae & $\mathrm{T}$ & SEU-atl & & $3,16,69$ & \\
\hline Bromus secalinus L. & Poaceae & $\mathrm{T}$ & EUAS & & 53 & \\
\hline Bromus squarrosus L. & Poaceae & $\mathrm{T}$ & SEU-po & & $5,8,21,23,25,35,38,43,53,55$ & V\&al-2019 \\
\hline Bromus sterilis L. & Poaceae & $\mathrm{T}$ & WISP & & $\begin{array}{c}4,11,14,16,17,20,21,28,29,30,32 \\
37,40,42,43,44,45,47,48,50,55,58 \\
60,63,69,70\end{array}$ & Ma-1992; V\&al-2019 \\
\hline Broussonetia papyrifera (L.) Vent. & Moraceae & $\mathrm{P}$ & CUAD & inv & $1,5,22,23,25,27,28,30$ & \\
\hline $\begin{array}{l}\text { Bryonia dioica Jacq. ssp. dioica } \\
\text { (Jacq.) Tutin }\end{array}$ & Cucurbitaceae & G & SEU-me & & $2,3,5,14,16,20,21,23,24,30,37$ & $\begin{array}{l}\text { Pe-1832:22, Vi-1852:138 } \\
\text { as B. dioica Jacq. }\end{array}$ \\
\hline $\begin{array}{l}\text { Bunium alpinum Waldst. et Kit. } \\
\text { ssp. montanum (W. D. J. Koch) P. } \\
\text { W. Ball }\end{array}$ & Apiaceae & G & ME-ilade & & $25,36,39,41,51,52,56,58$ & V\&al-2019 \\
\hline Bunium ferulaceum Sibth. et Sm. & Apiaceae & G & EEUPO & & $8,9,11$ & \\
\hline Buphthalmum salicifolium L. & Asteraceae & $\mathrm{H}$ & $\mathrm{CEU}$ & & 3 & Vi-1847:66; V\&al-2019 \\
\hline Bupleurum praealtum $\mathrm{L}$ & Apiaceae & $\mathrm{T}$ & SEU-me & & $4,5,11$ & \\
\hline Bupleurum veronense Turra & Apiaceae & $\mathrm{T}$ & ME-ilseu & & $\begin{array}{c}1,2,7,8,17,18,19,23,25,26,27,29 \\
30,41,43,44,47,48,49,51,54,57,58 \\
59,60,61 \\
\end{array}$ & LRS-1989; V\&al-2019 \\
\hline Calamagrostis epigejos (L.) Roth & Poaceae & $\mathrm{H}$ & WISP & & 53 & V\&al-2019 \\
\hline $\begin{array}{l}\text { Calamintha glandulosa (Req.) } \\
\text { Benth. }\end{array}$ & Lamiaceae & $\mathrm{H}$ & ME-circ & & 47 & \\
\hline $\begin{array}{l}\text { Calamintha grandiflora (L.) } \\
\text { Moench }\end{array}$ & Lamiaceae & $\mathrm{H}$ & SEU-mo & & $19,22,37 ?, 37,42$ & \\
\hline Calamintha nepetoides Jord. & Lamiaceae & $\mathrm{H}$ & SEU-po & & $3,5,13,37$ & \\
\hline Calendula officinalis L. & Asteraceae & $\mathrm{T}$ & CUAD & & $1,42^{*}$ & \\
\hline Calepina irregularis (Asso) Thell. & Brassicaceae & $\mathrm{T}$ & EURO & & 61,69 & V\&al-2019 \\
\hline Calystegia sepium (L.) R. Br. & Convolvulaceae & G & WISP & & $14,22,23,69,70$ & \\
\hline Campanula erinus L. & Campanulaceae & $\mathrm{T}$ & ME-circ & & 14 & \\
\hline Campanula glomerata $\mathrm{L}$. & Campanulaceae & $\mathrm{H}$ & EUAS & & 54 & Vi-1847:130; V\&al-2019 \\
\hline Campanula persicifolia $\mathrm{L}$ & Campanulaceae & $\mathrm{H}$ & EUAS & & $11,32,40,41,65$ & V\&al-2019 \\
\hline Campanula pyramidalis $\mathrm{L}$. & Campanulaceae & $\mathrm{H}$ & ME-ilade & & $11,31,32,34,35,36,39,40,52$ & VRZ-2012; V\&al-2019 \\
\hline Campanula rapunculoides L. & Campanulaceae & $\mathrm{H}$ & EUAS & & Significant Landscape of Sutina & V\&al-2019 \\
\hline Campanula rapunculus L. & Campanulaceae & $\mathrm{H}$ & EUAS & & $8,11,19,25,26,39,51,55,58,65$ & V\&al-2019 \\
\hline Campanula rotundifolia $\mathrm{L}$. & Campanulaceae & $\mathrm{H}$ & EURO & & 32 & \\
\hline Campanula scheuchzeri Vill. & Campanulaceae & $\mathrm{H}$ & SEU-me & & 33 & \\
\hline $\begin{array}{l}\text { Campanula sibirica L. ssp. } \\
\text { divergentiformis (Jáv.) Domin }\end{array}$ & Campanulaceae & $\mathrm{H}$ & SEEU & & $\begin{array}{c}1,24,26,31,37,38,39,40,46,47,57 \\
61,62,63,64,65,69,70\end{array}$ & $\begin{array}{l}\text { VRZ-2012, as C. sibirica } \\
\text { L.; V\&al-2019 }\end{array}$ \\
\hline
\end{tabular}




\begin{tabular}{|c|c|c|c|c|c|c|}
\hline Taxon & Family & \begin{tabular}{|c|} 
Life \\
form
\end{tabular} & Geoelement & Status & Localities (finding sites) & $\begin{array}{l}\text { Previously recorded } \\
\text { findings }\end{array}$ \\
\hline $\begin{array}{l}\text { Campanula trachelium L. ssp. } \\
\text { trachelium }\end{array}$ & Campanulaceae & $\mathrm{H}$ & EUAS & DD & $11,21,32,35,38,40,58,59,63,65$ & $\begin{array}{l}\text { Vi-1847:131; Je-1952; } \\
\text { V\&al-2019 as C. } \\
\text { trachelium L. }\end{array}$ \\
\hline Campsis radicans (L.) Seen. & Bignoniaceae & $\mathrm{P}$ & CUAD & & $22 *, 23$ & \\
\hline Capsella bursa-pastoris (L.) Medik. & Brassicaceae & $\mathrm{H}$ & WISP & & $3,9,13,14,16,27,49,50,65,66$ & V\&al-2019 \\
\hline Capsella rubella Reut. & Brassicaceae & $\mathrm{T}$ & ME-circ & & $\begin{array}{c}1,2,5,11,14,16,20,24,29,30,37,42 \\
44,47,61,66,69,70\end{array}$ & V\&al-2019 \\
\hline Cardamine graeca $\mathrm{L}$. & Brassicaceae & $\mathrm{T}$ & SEU-me & & 14 & V\&al-2019 \\
\hline Cardamine hirsuta $\mathrm{L}$. & Brassicaceae & $\mathrm{T}$ & WISP & & $2,20,37,60,61$ & V\&al-2019 \\
\hline $\begin{array}{l}\text { Cardaria draba (L.) Desv. ssp. } \\
\text { draba }\end{array}$ & Brassicaceae & $\mathrm{T}$ & WISP & & $29,44,47,63$ & $\begin{array}{l}\text { V\&al-2019 as C. draba } \\
\text { (L.) Desv. }\end{array}$ \\
\hline Carduus acanthoides L. & Asteraceae & $\mathrm{H}$ & SEU-co & & 59 & \\
\hline Carduus candicans Waldst. et Kit. & Asteraceae & $\mathrm{H}$ & SEEU & & $11,25,65$ & Vi-1847:47 \\
\hline $\begin{array}{l}\text { Carduus micropterus (Borbás) } \\
\text { Teyber ssp. micropterus }\end{array}$ & Asteraceae & $\mathrm{H}$ & ME-ilade & $\begin{array}{l}\text { end, } \\
\text { sp }\end{array}$ & $\begin{array}{c}1,2,6,7,8,9,15,17,21,25,33,38,40 \\
41,43,46,56,58,62,65\end{array}$ & $\begin{array}{l}\text { V\&al-2019, as C. } \\
\text { micropterus (Borbás) } \\
\text { Teyber }\end{array}$ \\
\hline $\begin{array}{l}\text { Carduus pycnocephalus L. ssp. } \\
\text { pycnocephalus }\end{array}$ & Asteraceae & $\mathrm{T}$ & ME-circ & DD & $1,3,5,23,25,59$ & $\begin{array}{l}\text { V\&al-2019, as C. } \\
\text { pycnocephalus L. }\end{array}$ \\
\hline Carex appropinquata Schumach. & Cyperaceae & $\mathrm{H}$ & EUAS & $\begin{array}{c}\mathrm{DD} \\
\mathrm{sp}\end{array}$ & Svilaja mountain range & L\&R-1989 \\
\hline Carex caryophyllea Latourr. & Cyperaceae & $\mathrm{H}$ & EUAS & & $2,13,37,42,44,67,70$ & \\
\hline Carex digitata $\mathrm{L}$. & Cyperaceae & $\mathrm{H}$ & EUAS & & 64 & Vi-1842:102 \\
\hline Carex distans $\mathrm{L}$. & Cyperaceae & $\mathrm{H}$ & ME-circ & & $23,28,46,47,70$ & V\&al-2019 \\
\hline Carex divisa Huds. & Cyperaceae & $\mathrm{H}$ & SEU-me & EN, sp & 46 & \\
\hline Carex divulsa Stokes ssp. divulsa & Cyperaceae & $\mathrm{H}$ & WISP & & $3,16,20,21,25,28,37,43,45,65$ & V\&al-2019 as C. divulsa \\
\hline $\begin{array}{l}\text { Carex divulsa Stokes ssp. leersii } \\
\text { (Kneuck.) W. Koch }\end{array}$ & Cyperaceae & $\mathrm{H}$ & EUAS & & 14,70 & \\
\hline Carex echinata Murray & Cyperaceae & $\mathrm{H}$ & $\mathrm{CIHO}$ & EN, sp & Significant Landscape of Sutina & V\&al-2019 \\
\hline Carex flacca Schreb. ssp. flacca & Cyperaceae & $\mathrm{H}$ & WISP & & $28,46,70$ & V\&al-2019 as C. flacca \\
\hline $\begin{array}{l}\text { Carex flacca Schreb. ssp. serrulata } \\
\text { (Biv.) Greuter }\end{array}$ & Cyperaceae & $\mathrm{H}$ & ME-circ & & $37,42,47,60,64,69$ & \\
\hline Carex hallerana Asso & Cyperaceae & $\mathrm{H}$ & SEU-me & & $29,30,31,37,57,61,64,69$ & Je-1952; V\&al-2019 \\
\hline Carex hirta L. & Cyperaceae & G & EUAS & & $14,48,70$ & V\&al-2019 \\
\hline Carex humilis Leyss. & Cyperaceae & $\mathrm{H}$ & EUAS & & $8,9,31,35,51,54,59,64$ & \\
\hline Carex kitaibeliana Degen ex Bech. & Cyperaceae & $\mathrm{H}$ & SEU-mo & & 34 & L\&R-1989; LRS-1989 \\
\hline Carex otrubae Podp. & Cyperaceae & $\mathrm{H}$ & SEU-me & & 28 & \\
\hline Carex pendula Huds. & Cyperaceae & $\mathrm{H}$ & EUAS & & Significant Landscape of Sutina & V\&al-2019 \\
\hline Carex riparia Curtis & Cyperaceae & G & EUAS & $\begin{array}{l}\text { VU, } \\
\text { sp }\end{array}$ & 70 & \\
\hline Carex spicata Huds. & Cyperaceae & $\mathrm{H}$ & EUAS & & $3,14,17,20,28,42,46,48,70$ & \\
\hline Carex supina Willd. ex Wahlenb. & Cyperaceae & G & SEU-po & $\begin{array}{c}\mathrm{DD} \\
\mathrm{sp}\end{array}$ & 52 & \\
\hline Carex vulpina L. & Cyperaceae & $\mathrm{H}$ & EUAS & & Significant Landscape of Sutina & V\&al-2019 \\
\hline $\begin{array}{l}\text { Carlina acanthifolia All. ssp. } \\
\text { acanthifolia }\end{array}$ & Asteraceae & $\mathrm{H}$ & SEU-me & DD & $7,12,40,52$ & $\begin{array}{l}\text { V\&al-2019 as C. } \\
\text { acanthifolia All. }\end{array}$ \\
\hline Carlina acaulis L. ssp. acaulis & Asteraceae & $\mathrm{H}$ & SEU-mo & & $41,57,62$ & \\
\hline $\begin{array}{l}\text { Carlina acaulis L. ssp. simplex } \\
\text { (Wettst. et Kit.) Nyman }\end{array}$ & Asteraceae & $\mathrm{H}$ & ILBA-end & & 9 & \\
\hline Carlina corymbosa L. & Asteraceae & $\mathrm{H}$ & ME-circ & & $2,5,18,21,22,25,26,43,45,64,66,70$ & V\&al-2019 \\
\hline Carlina vulgaris L. & Asteraceae & $\mathrm{H}$ & EUAS & & $\begin{array}{c}4,7,8,9,10,11,13,18,19,21,22,30 \\
31,33,34,35,39,40,48,51,53,54,55 \\
56,57,58,59,65,69\end{array}$ & \\
\hline Carpinus betulus L. & Corylaceae & $\mathrm{P}$ & CEU & & "in monte Svilaja" & Vi-1842:210 \\
\hline Carpinus orientalis Mill. & Corylaceae & $\mathrm{P}$ & ME-ilseu & & \begin{tabular}{|c|}
$1,2,13,14,15,16,17,18,19,20,22$ \\
$23,26,27,29,30,31,37,43,47,49,52$ \\
$53,60,61,64,67,69,70$
\end{tabular} & $\begin{array}{l}\text { Je-1952; Me-1972; } \\
\text { L\&al-1987; V\&al-2019 }\end{array}$ \\
\hline Carthamus lanatus L. ssp. lanatus & Asteraceae & $\mathrm{T}$ & ME-circ & & $1,3,5,17,21,23,28,43,46$ & $\begin{array}{l}\text { V\&al-2019 as C. lanatus } \\
\text { L. }\end{array}$ \\
\hline Carum carvi L. & Apiaceae & $\mathrm{H}$ & EUAS & & 70 & \\
\hline Celtis australis L. & Ulmaceae & $\mathrm{P}$ & SEU-me & & $\begin{array}{c}1,2,4,5,6,20,22,23,25,26,27,28 \\
29,30,43,45,50\end{array}$ & L\&al-1987; V\&al-2019 \\
\hline
\end{tabular}




\begin{tabular}{|c|c|c|c|c|c|c|}
\hline Taxon & Family & \begin{tabular}{|c|} 
Life \\
form
\end{tabular} & Geoelement & Status & Localities (finding sites) & $\begin{array}{l}\text { Previously recorded } \\
\text { findings }\end{array}$ \\
\hline Centaurea bracteata Scop. & Asteraceae & $\mathrm{H}$ & ME-west & & 70 & LRS-1989 \\
\hline Centaurea calcitrapa $\mathrm{L}$. & Asteraceae & $\mathrm{T}$ & ME-atl & & 25 & \\
\hline Centaurea cyanus L. & Asteraceae & $\mathrm{T}$ & SEEU & & 37,67 & \\
\hline $\begin{array}{l}\text { Centaurea deusta Ten. ssp. concolor } \\
\text { (DC.) Hayek }\end{array}$ & Asteraceae & $\mathrm{H}$ & ME-eu & & $18,32,38,39,40,41$ & \\
\hline Centaurea fritschii Hayek & Asteraceae & $\mathrm{H}$ & ILBA-end & & Significant Landscape of Sutina & V\&al-2019 \\
\hline $\begin{array}{l}\text { Centaurea glaberrima Tausch ssp. } \\
\text { divergens (Vis.) Hayek }\end{array}$ & Asteraceae & $\mathrm{H}$ & ME-ilade & & $\begin{array}{l}\text { on the dolomites of the southern } \\
\text { slopes of Svilaja near Muć; SL Sutina }\end{array}$ & $\begin{array}{l}\text { L\&al-1987 as C. huterii } \\
\text { Hay. (C. petteri auct. } \\
\text { p.p.); V\&al-2019 }\end{array}$ \\
\hline Centaurea jacea L. & Asteraceae & $\mathrm{H}$ & EUAS & & 32 & \\
\hline Centaurea nicolae Bald. & Asteraceae & $\mathrm{H}$ & ME-ilade & $\begin{array}{l}\text { end, } \\
\text { DD, } \\
\text { sp }\end{array}$ & Svilaja mountain range & $\begin{array}{l}\text { LRS-1989, as C. } \\
\text { lanceolata (Vis.) Vuk. (C. } \\
\text { nikolai) }\end{array}$ \\
\hline $\begin{array}{l}\text { Centaurea pannonica (Heuff.) } \\
\text { Simonk. }\end{array}$ & Asteraceae & $\mathrm{H}$ & EEUPO & & $18,19,59,62,68$ & \\
\hline Centaurea rupestris L. & Asteraceae & $\mathrm{H}$ & ME-ilape & & $8,9,26,30,31$ & L\&al-1987 \\
\hline Centaurea scabiosa L & Asteraceae & $\mathrm{H}$ & EUAS & & $4,13,15,65$ & Vi-1847:34 \\
\hline Centaurea solstitialis L. & Asteraceae & $\mathrm{H}$ & SEU-po & & $2,4,5,22,23,25,27,43$ & \\
\hline $\begin{array}{l}\text { Centaurea spinosociliata Seenus } \\
\text { ssp. cristata (Bertol.) Dostál }\end{array}$ & Asteraceae & $\mathrm{H}$ & ME-ilade & $\begin{array}{c}\text { end, } \\
\text { NT, sp }\end{array}$ & $\begin{array}{c}1,5,9,13,15,19,21,23,25,26,27,31 \\
35,43,46,49,65\end{array}$ & $\begin{array}{l}\text { L\&al-1987, as C. cristata } \\
\text { Bartl. ssp. cristata s.s.; } \\
\text { V\&al-2019 }\end{array}$ \\
\hline $\begin{array}{l}\text { Centaurea spinosociliata Seenus } \\
\text { ssp. spinosociliata }\end{array}$ & Asteraceae & $\mathrm{H}$ & ME-ilade & $\begin{array}{c}\text { end, } \\
\text { NT, sp }\end{array}$ & $31,49,51$ & $\begin{array}{l}\text { Tr-1992, as C. } \\
\text { spinoso-ciliata Seen. }\end{array}$ \\
\hline Centaurea triumfetti All. & Asteraceae & $\mathrm{H}$ & SEU-mo & $\mathrm{DD}$ & $11,13,14,17,24,25,31,34$ & $\begin{array}{l}\text { LRS-1989, as C. cana Sb. } \\
\& \text { Sm.; V\&al-2019 }\end{array}$ \\
\hline Centaurea tuberosa Vis. & Asteraceae & G & ILBA-end & $\begin{array}{l}\text { end, } \\
\text { sp }\end{array}$ & $2,11,29,36,41,52,67$ & $\begin{array}{l}\text { Ja-1908; L\&al-1987; } \\
\text { V\&al-2019 }\end{array}$ \\
\hline Centaurea weldeniana Rchb. & Asteraceae & $\mathrm{H}$ & ME-ilade & & $4,7,15,22,31$ & \\
\hline Centaurium erythraea Rafn & Gentianaceae & $\mathrm{T}$ & WISP & & $\begin{array}{c}5,8,11,15,21,22,24,25,31,34,35 \\
40,45,50,51,53,56,59,65\end{array}$ & V\&al-2019 \\
\hline Centranthus ruber (L.) DC. & Valerianaceae & $\mathrm{Ch}$ & ME-atl & & $1,23,27$ & \\
\hline $\begin{array}{l}\text { Cephalanthera damasonium (Mill.) } \\
\text { Druce }\end{array}$ & Orchidaceae & G & SEU-me & NT, sp & 41,42 & $\begin{array}{l}\text { Je-1952, as C. alba; } \\
\text { Kr-2005:43; Df-2006; } \\
\text { V\&al-2019 }\end{array}$ \\
\hline $\begin{array}{l}\text { Cephalanthera longifolia (L.) } \\
\text { Fritsch }\end{array}$ & Orchidaceae & G & EUAS & NT, sp & 32,45 & Kr-2005:48; Df-2006 \\
\hline Cephalanthera rubra (L.) Rich. & Orchidaceae & G & EUAS & NT, sp & 32 & V\&al-2019 \\
\hline $\begin{array}{l}\text { Cephalanthera } \times \text { schulzei E.G. } \\
\text { Camus, Bergon, A. Camuis (C. } \\
\text { damasonium } \times \text { C. longifolia })^{* *}\end{array}$ & Orchidaceae & G & EUAS & $\mathrm{sp}$ & $\begin{array}{c}\text { site } 263 \text { (XJ2345) SO Zelovo, 820-840 } \\
\text { m }\end{array}$ & Df-2006 \\
\hline $\begin{array}{l}\text { Cephalaria leucantha (L.) Roem. et } \\
\text { Schult. }\end{array}$ & Dipsacaceae & $\mathrm{H}$ & ME-circ & & $\begin{array}{c}1,2,6,12,13,22,25,26,31,40,41,46 \\
51,64,70\end{array}$ & $\begin{array}{l}\text { Tr-1992; Se\&al-2016; } \\
\text { V\&al-2019 }\end{array}$ \\
\hline $\begin{array}{l}\text { Cerastium brachypetalum Pers. } \\
\text { ssp. brachypetalum }\end{array}$ & Caryophyllaceae & $\mathrm{T}$ & SEU-mo & & $11,20,35,36,44$ & V\&al-2019 \\
\hline $\begin{array}{l}\text { Cerastium brachypetalum Pers. } \\
\text { ssp. roeseri (Bois. et Heldr.) } \\
\text { Nyman }\end{array}$ & Caryophyllaceae & $\mathrm{T}$ & SEU-atl & & $29,36,37,39,41,42,45,69,70$ & \\
\hline $\begin{array}{l}\text { Cerastium brachypetalum Pers. } \\
\text { ssp. tenoreanum (Ser,) Soo }\end{array}$ & Caryophyllaceae & $\mathrm{T}$ & SEU-me & & 11 & \\
\hline $\begin{array}{l}\text { Cerastium fontanum Baumg. ssp. } \\
\text { vulgare (Hartman) Greuter et } \\
\text { Burdet }\end{array}$ & Caryophyllaceae & $\mathrm{H}$ & $\mathrm{CIHO}$ & & 28 & V\&al-2019 \\
\hline Cerastium glomeratum Thuill. & Caryophyllaceae & $\mathrm{T}$ & WISP & & $17,44,60$ & V\&al-2019 \\
\hline $\begin{array}{l}\text { Cerastium grandiflorum Waldst. et } \\
\text { Kit. }\end{array}$ & Caryophyllaceae & $\mathrm{H}$ & ILBA-end & $\begin{array}{c}\text { end, } \\
\text { sp }\end{array}$ & $9,10,32,33,34,35,38,39,40,41$ & Ja-1908; LRS-1989 \\
\hline $\begin{array}{l}\text { Cerastium ligusticum Viv. ssp. } \\
\text { trichogynum (Moschl) P.D.Sell. et } \\
\text { Whitehead }\end{array}$ & Caryophyllaceae & $\mathrm{T}$ & ME-ilade & & 6,52 & V\&al-2019 \\
\hline $\begin{array}{l}\text { Cerastium pumilum Curtis ssp. } \\
\text { glutinosum (Fries) Jalas }\end{array}$ & Caryophyllaceae & $\mathrm{T}$ & WISP & & $\begin{array}{c}2,6,9,17,37,42,47,50,51,52,55,57 \\
58,63,69,70\end{array}$ & \\
\hline Cerastium semidecandrum $\mathrm{L}$. & Caryophyllaceae & $\mathrm{T}$ & SEU-po & & 42 & \\
\hline Cerastium tomentosum $\mathrm{L}$. & Caryophyllaceae & $\mathrm{H}$ & CUAD & & $1,20,37$ & \\
\hline
\end{tabular}




\begin{tabular}{|c|c|c|c|c|c|c|}
\hline Taxon & Family & \begin{tabular}{|c|} 
Life \\
form
\end{tabular} & Geoelement & Status & Localities (finding sites) & $\begin{array}{l}\text { Previously recorded } \\
\text { findings }\end{array}$ \\
\hline $\begin{array}{l}\text { Chaenorhinum minus (L.) Lange } \\
\text { ssp. litorale (Willd.) Hayek }\end{array}$ & Scrophulariaceae & $\mathrm{T}$ & ME-ilape & & 50 & \\
\hline $\begin{array}{l}\text { Chaenorhinum minus (L.) Lange } \\
\text { ssp. minus }\end{array}$ & Scrophulariaceae & $\mathrm{T}$ & EURO & & $1,6,9,11,27,38,50,68$ & V\&al-2019 \\
\hline Chaerophyllum aureum $\mathrm{L}$. & Apiaceae & $\mathrm{H}$ & SEU-co & & 37,47 & \\
\hline Chaerophyllum bulbosum L. & Apiaceae & $\mathrm{H}$ & EUAS & & $16,20,65$ & $\begin{array}{l}\text { Vi-1852:65, as C. } \\
\text { laevigatum Vis. }\end{array}$ \\
\hline Chaerophyllum coloratum $\mathrm{L}$ & Apiaceae & $\mathrm{H}$ & ME-ilade & $\begin{array}{c}\text { end, } \\
\text { NT, sp }\end{array}$ & $1,5,17,20,25,28,37,45,65$ & V\&al-2019 \\
\hline Chaerophyllum temulum L. & Apiaceae & $\mathrm{H}$ & EUAS & & $14,16,37,42$ & Vi-1852:66; Ma-1992 \\
\hline $\begin{array}{l}\text { Chamaecytisus falcatus (Waldst. et } \\
\text { Kit.) Holub }\end{array}$ & Fabaceae & $\mathrm{Ch}$ & CEU & & $10,11,19,30,32,34,35,64$ & \\
\hline Chamaecytisus hirsutus (L.) Link & Fabaceae & $\mathrm{Ch}$ & SEU-po & & 11,67 & V\&al-2019 \\
\hline $\begin{array}{l}\text { Chamaespartium sagittale (L.) } \\
\text { Gibbs }\end{array}$ & Fabaceae & $\mathrm{Ch}$ & SEU-me & & $13,34,55$ & \\
\hline Chamomilla recutita (L.) Rauschert & Asteraceae & $\mathrm{T}$ & CUAD & & 42 & \\
\hline $\begin{array}{l}\text { Chamomilla suaveolens (Pursh) } \\
\text { Rydb. }\end{array}$ & Asteraceae & $\mathrm{T}$ & CUAD & inv & $16,23,28$ & \\
\hline Chelidonium majus L. & Papaveraceae & $\mathrm{H}$ & WISP & & $11,14,16,20,30,65,69$ & Pe-1832:34, Ma-1992 \\
\hline Chenopodium album $\mathrm{L}$. & Chenopodiaceae & $\mathrm{T}$ & WISP & & $1,4,6,13,14,22,25,27,49,50,65,69$ & V\&al-2019 \\
\hline Chenopodium hybridum $\mathrm{L}$. & Chenopodiaceae & $\mathrm{T}$ & $\mathrm{CIHO}$ & & 14 & \\
\hline Chenopodium murale L. & Chenopodiaceae & $\mathrm{T}$ & WISP & DD & 49 & \\
\hline Chenopodium strictum Roth & Chenopodiaceae & $\mathrm{T}$ & WISP & DD & $3,13,22,24,27$ & \\
\hline Chenopodium vulvaria $\mathrm{L}$. & Chenopodiaceae & $\mathrm{T}$ & SEU-me & DD & 14,68 & \\
\hline Chondrilla juncea $\mathrm{L}$. & Cichoriaceae & $\mathrm{H}$ & EUAS & & $\begin{array}{c}1,3,4,5,13,14,15,17,21,23,24,25 \\
27,29,37,43,45,46,50,54,55,70\end{array}$ & V\&al-2019 \\
\hline Chouardia lakusicii (Šilić) Speta & Asparagaceae & G & ME-ilade & $\begin{array}{l}\text { end, } \\
\text { sp }\end{array}$ & on the southern slopes of Svilaja & Se-2016 \\
\hline $\begin{array}{l}\text { Chouardia litardierei (Breistr.) } \\
\text { Speta }\end{array}$ & Asparagaceae & G & ME-ilade & $\begin{array}{c}\text { end, } \\
\text { NT, sp }\end{array}$ & Significant Landscape of Sutina & V\&al-2019 \\
\hline Chrysopogon gryllus (L.) Trin. & Poaceae & $\mathrm{H}$ & ME-po & & $\begin{array}{c}1,2,5,6,18,22,23,25,28,31,43,46 \\
48,69\end{array}$ & Tr-1992; V\&al-2019 \\
\hline Cichorium intybus L. & Cichoriaceae & $\mathrm{H}$ & WISP & & $\begin{array}{c}1,3,4,6,7,8,13,14,15,16,17,18,19 \\
21,22,23,25,26,27,28,31,32,35,38 \\
40,43,46,47,50,51,53,54,55,57,58 \\
59,60,61,65,66,68\end{array}$ & V\&al-2019 \\
\hline Cirsium acaule Scop. ssp. acaule & Asteraceae & $\mathrm{H}$ & EUAS & & $8,33,59$ & $\begin{array}{l}\text { Vi-1847:50, V\&al-2019, } \\
\text { as C. acaule Scop. }\end{array}$ \\
\hline Cirsium arvense (L.) Scop. & Asteraceae & $\mathrm{T}$ & EUAS & & $1,4,13,16,27,28,35,40,46,66,68,70$ & V\&al-2019 \\
\hline $\begin{array}{l}\text { Cirsium eriophorum (L.) Scop. ssp. } \\
\text { eriophorum }\end{array}$ & Asteraceae & $\mathrm{T}$ & SEU-co & & 32 & \\
\hline Cirsium vulgare (Savi) Ten. & Asteraceae & $\mathrm{H}$ & EUAS & & $\begin{array}{c}1,2,4,5,8,10,11,13,16,17,18,21 \\
22,23,27,28,30,32,35,37,40,44,45 \\
46,47,48,50,54,57,58,59,65,68,69 \\
70\end{array}$ & V\&al-2019 \\
\hline Cleistogenes serotina (L.) Keng & Poaceae & $\mathrm{H}$ & SEU-po & & 18,25 & \\
\hline Clematis flammula $\mathrm{L}$. & Ranunculaceae & $P$ & ME-circ & & $\begin{array}{c}1,2,4,5,6,13,14,15,16,17,18,19 \\
20,22,23,25,27,28,29,30,37,39,42 \\
43,44,45,46,47,48,60,70\end{array}$ & V\&al-2019 \\
\hline Clematis recta $\mathrm{L}$. & Ranunculaceae & $\mathrm{H}$ & EUAS & & 31,35 & V\&al-2019 \\
\hline Clematis vitalba $\mathrm{L}$. & Ranunculaceae & $\mathrm{P}$ & EURO & & $\begin{array}{c}1,3,4,13,14,15,16,20,21,22,23,25 \\
26,27,31,32,34,35,36,37,38,40,42, \\
43,47,48,50,53,54,55,57,58,59,61 \\
62,63,64,65,66,69\end{array}$ & $\begin{array}{l}\text { L\&al-1987; Ma-1992; } \\
\text { V\&al-2019 }\end{array}$ \\
\hline Clematis viticella $\mathrm{L}$. & Ranunculaceae & $\mathrm{H}$ & SEU-po & & $3,16,22,23,28$ & \\
\hline Clinopodium vulgare $\mathrm{L}$. & Lamiaceae & $\mathrm{H}$ & WISP & & $4,11,18,19,21,26,32,38,40,64,65$ & V\&al-2019 \\
\hline $\begin{array}{l}\text { Cnidium silaifolium (Jacq.) } \\
\text { Simonk. }\end{array}$ & Apiaceae & $\mathrm{H}$ & SEU-me & & 11,32 & $\begin{array}{l}\text { Vi-1852:46 as C. apioides } \\
\text { Spr. }\end{array}$ \\
\hline Coeloglossum viride (L.) Hartm. & Orchidaceae & G & $\mathrm{CIHO}$ & $\mathrm{sp}$ & on the southern slopes of Svilaja & Se-2016 \\
\hline Colchicum autumnale $\mathrm{L}$. & Colchicaceae & G & CEU & & $\begin{array}{c}13,17,18,19,27,29,37,47,49,51,54 \\
60\end{array}$ & V\&al-2019 \\
\hline Colchicum hungaricum Janka & Colchicaceae & G & SEEU & & $2,5,61$ & V\&al-2019 \\
\hline
\end{tabular}




\begin{tabular}{|c|c|c|c|c|c|c|}
\hline Taxon & Family & \begin{tabular}{|c|} 
Life \\
form
\end{tabular} & Geoelement & Status & Localities (finding sites) & $\begin{array}{l}\text { Previously recorded } \\
\text { findings }\end{array}$ \\
\hline Colchicum kochii Parl. & Colchicaceae & G & ILBA-end & & $13,41,42$ & \\
\hline Colchicum visianii Parl. & Colchicaceae & G & ME-ilape & & 25 & \\
\hline Colutea arborescens L. & Fabaceae & P & ME-circ & & $\begin{array}{c}1,16,17,21,24,26,31,42,43,45,46 \\
48,55,61,62\end{array}$ & V\&al-2019 \\
\hline Conium maculatum L. & Apiaceae & $\mathrm{H}$ & WISP & & $14,16,17,25,67,70$ & Pe-1832:39; Vi-1852:69 \\
\hline Consolida regalis S.F.Gray & Ranunculaceae & $\mathrm{T}$ & SEU-me & & $3,4,23,26,28$ & V\&al-2019 \\
\hline Convallaria majalis $\mathrm{L}$. & Asparagaceae & G & $\mathrm{CIHO}$ & & 32 & \\
\hline Convolvulus arvensis $\mathrm{L}$. & Convolvulaceae & G & WISP & & $\begin{array}{c}1,3,4,5,9,11,13,14,15,16,20,21 \\
22,23,25,27,28,29,36,37,39,40,43 \\
44,45,47,49,50,55,58,59,61,65,66 \\
68,69,70\end{array}$ & V\&al-2019 \\
\hline Convolvulus cantabrica $\mathrm{L}$. & Convolvulaceae & $\mathrm{H}$ & SEU-me & & $1,2,5,21,23,26,43,46,47$ & V\&al-2019 \\
\hline Conyza bonariensis (L.) Cronquist & Asteraceae & $\mathrm{T}$ & CUAD & inv & 22 & \\
\hline Conyza canadensis (L.) Cronquist & Asteraceae & $\mathrm{T}$ & CUAD & inv & $4,13,14,15,22,44,49,50,65,66$ & \\
\hline $\begin{array}{l}\text { Conyza sumatrensis (Retz.) E. } \\
\text { Walker }\end{array}$ & Asteraceae & $\mathrm{T}$ & CUAD & inv & $1,4,13,14,22$ & \\
\hline Corallorhiza trifida Châtel. & Orchidaceae & G & $\mathrm{CIHO}$ & $\mathrm{sp}$ & "in monte Svilaja"; Sinjsko Zelovo & $\begin{array}{l}\text { Vi-1842:182 as C. innata } \\
\text { R. Br.; Kr-2005:54 }\end{array}$ \\
\hline Cornus mas L. & Cornaceae & $P$ & SEU-co & & $\begin{array}{c}1,2,5,7,14,15,16,17,18,19,20,22, \\
25,26,27,30,31,36,37,40,41,42,44 \\
47,48,49,54,55,57,58,59,60,61,62, \\
63,64,65,67,69,70\end{array}$ & $\begin{array}{l}\text { Je-1952; L\&al-1987; } \\
\text { V\&al-2019 }\end{array}$ \\
\hline Cornus sanguinea L. & Cornaceae & P & EURO & & $\begin{array}{c}1,4,14,16,20,22,25,28,30,31,37 \\
42,43,44,45,46,47,50,57,59,61,62 \\
64,66,69,70\end{array}$ & V\&al-2019 \\
\hline $\begin{array}{l}\text { Coronilla emerus L. ssp. emeroides } \\
\text { Boiss. et Spruner }\end{array}$ & Fabaceae & $\mathrm{P}$ & ME-east & & $\begin{array}{c}1,2,5,19,21,25,29,30,32,36,37,39 \\
41,42,43,44,46,47,50,60,61,64,69 \\
70\end{array}$ & $\begin{array}{l}\text { Je-1952 and L\&al-1987 } \\
\text { as C. emeroides Boiss. et } \\
\text { Spruner; V\&al-2019 }\end{array}$ \\
\hline Coronilla scorpioides (L.) Koch & Fabaceae & $\mathrm{T}$ & ME-circ & & $23,25,29,43,45$ & \\
\hline Coronilla varia $\mathrm{L}$. & Fabaceae & $\mathrm{H}$ & EURO & & $\begin{array}{c}1,11,13,15,16,17,21,22,25,27,28 \\
32,35,40,43,46,55,58,62,65,66\end{array}$ & V\&al-2019 \\
\hline $\begin{array}{l}\text { Coronopus squamatus (Forssk.) } \\
\text { Asch. }\end{array}$ & Brassicaceae & $\mathrm{T}$ & WISP & & 14 & $\begin{array}{l}\text { Ma-1964 as C. } \\
\text { procumbens Gilib. }\end{array}$ \\
\hline Corydalis ochroleuca Koch & Fumariaceae & $\mathrm{H}$ & ME-ilseu & & $1,10,32,34,35,40,52$ & \\
\hline Corydalis solida (L.) Swartz & Fumariaceae & G & EUAS & & $11,15,41,66,67$ & Mi\&al-2013; V\&al-2019 \\
\hline Corylus avellana $\mathrm{L}$ & Corylaceae & $\mathrm{P}$ & EURO & & $13^{*}, 14,37,40,44,52,63,65,66,70$ & $\begin{array}{l}\text { Pe-1832:41; Je-1952; } \\
\text { L\&al-1987, as C. pontica } \\
\text { Koch.; V\&al-2019 }\end{array}$ \\
\hline Cotinus coggygria Scop. & Anacardiaceae & $\mathrm{P}$ & SEU-po & & $2,26,27,30,46,68,70$ & L\&al-1987; V\&al-2019 \\
\hline Cotoneaster integerrimus Medik. & Rosaceae & $\mathrm{P}$ & EUAS & & $34,35,40,41$ & \\
\hline $\begin{array}{l}\text { Cotoneaster nebrodensis (Guss.) K. } \\
\text { Koch }\end{array}$ & Rosaceae & $P$ & SEU-po & & Significant Landscape of Sutina & V\&al-2019 \\
\hline Crataegus monogyna Jacq. & Rosaceae & $\mathrm{P}$ & EUAS & & $\begin{array}{c}2,3,4,5,6,7,8,9,11,13,15,17,18, \\
19,20,22,23,26,27,30,31,32,34,37 \\
38,39,40,41,43,44,45,46,47,48,49, \\
50,51,52,53,54,55,57,58,59,60,61, \\
63,64,65,67,69\end{array}$ & Je-1952; V\&al-2019 \\
\hline Crepis biennis L. & Cichoriaceae & $\mathrm{H}$ & CEU & & $47,60,61,65$ & \\
\hline Crepis capillaris (L.) Wallr. & Cichoriaceae & $\mathrm{T}$ & $\mathrm{CEU}$ & & Significant Landscape of Sutina & V\&al-2019 \\
\hline Crepis chondriloides Jacq. & Cichoriaceae & $\mathrm{H}$ & ME-ilade & & $8,31,34,35,39,41,51,52,53,58,67$ & Ja-1908; V\&al-2019 \\
\hline $\begin{array}{l}\text { Crepis foetida L. ssp. rhoeadifolia } \\
\text { (M. Bieb.) Čelak. }\end{array}$ & Cichoriaceae & $\mathrm{T}$ & SEU-me & & $\begin{array}{c}1,3,4,5,25,26,27,43,46,49,54,55 \\
59,68\end{array}$ & \\
\hline Crepis neglecta $\mathrm{L}$. & Cichoriaceae & $\mathrm{T}$ & ME-eu & & $\begin{array}{c}1,5,11,17,21,25,27,29,37,43,45 \\
46,59,69\end{array}$ & V\&al-2019 \\
\hline Crepis pannonica (Jacq.) C. Koch & Cichoriaceae & $\mathrm{T}$ & EEUPO & & 65 & \\
\hline Crepis pulchra $\mathrm{L}$. & Cichoriaceae & $\mathrm{T}$ & SEU-me & & $2,14,16$ & \\
\hline Crepis rubra $\mathrm{L}$. & Cichoriaceae & $\mathrm{T}$ & ME-east & & $2,30,45$ & De-2010; V\&al-2019 \\
\hline Crepis sancta (L.) Babc. & Cichoriaceae & $\mathrm{T}$ & ME-east & & $2,16,20,30,37,44,47,69,70$ & \\
\hline Crepis setosa Haller $\mathrm{f}$. & Cichoriaceae & $\mathrm{T}$ & SEU-po & & $3,5,14,43,59$ & \\
\hline $\begin{array}{l}\text { Crepis vesicaria L. ssp. taraxacifolia } \\
\text { (Thuill.) Thell. }\end{array}$ & Cichoriaceae & $\mathrm{T}$ & ME-atl & & $37,42,67,69,70$ & V\&al-2019 \\
\hline Crepis vesicaria L. ssp. vesicaria & Cichoriaceae & $\mathrm{T}$ & ME-circ & & 47,70 & \\
\hline
\end{tabular}




\begin{tabular}{|c|c|c|c|c|c|c|}
\hline Taxon & Family & \begin{tabular}{|c|} 
Life \\
form
\end{tabular} & Geoelement & Status & Localities (finding sites) & $\begin{array}{l}\text { Previously recorded } \\
\text { findings }\end{array}$ \\
\hline $\begin{array}{l}\text { Crocus biflorus Mill. ssp. weldenii } \\
\text { (Hoppeet Fürnr.) K. Richt. }\end{array}$ & Iridaceae & G & SEU-po & end & 8 & $\begin{array}{l}\text { Vi-1842:119 as C. biflorus } \\
\text { Mill.; Se-2016 }\end{array}$ \\
\hline Crocus purpureus Weston & Iridaceae & G & SEU-mo & & $5,10,11,15$ & \\
\hline $\begin{array}{l}\text { Crocus variegatus Hoppe et } \\
\text { Hornsch. }\end{array}$ & Iridaceae & G & ME-ilade & & Svilaja mountain range; SL Sutina & $\begin{array}{l}\text { L\&R-1989, as C. } \\
\text { reticulatus Stev.; } \\
\text { V\&al-2019 }\end{array}$ \\
\hline Crocus thomasii Ten. & Iridaceae & G & ME-ilape & $\begin{array}{c}\text { end, } \\
\text { sp }\end{array}$ & $26,27,48$ & \\
\hline Crocus tommasinianus Herb. & Iridaceae & G & ILBA-end & & Svilaja mountain range; SL Sutina & L\&R-1989 \\
\hline Cruciata glabra (L.) Ehrend & Rubiaceae & $\mathrm{H}$ & EUAS & & $19,32,53,64,65,67$ & $\begin{array}{l}\text { Vi-1852:9; Je-1952 as } \\
\text { Galium vernum Scop. }\end{array}$ \\
\hline Cruciata laevipes Opiz & Rubiaceae & $\mathrm{T}$ & EUAS & & $\begin{array}{c}2,13,16,20,36,37,42,44,46,47,48 \\
58,60,61,65,66,69,70\end{array}$ & V\&al-2019 \\
\hline $\begin{array}{l}\text { Cruciata pedemontana (Belliard) } \\
\text { Ehrend. }\end{array}$ & Rubiaceae & $\mathrm{T}$ & SEU-me & & $8,11,21$ & Vi-1852:9 \\
\hline Cupressus horizontalis Mill. & Cupressaceae & $\mathrm{P}$ & CUAD & & 29 & \\
\hline Cupressus sempervirens L. & Cupressaceae & $\mathrm{P}$ & CUAD & & $1,3,16,22 *, 46$ & \\
\hline Cuscuta campestris Yuncker & Cuscutaceae & $\mathrm{T}$ & CUAD & inv & 15,22 & \\
\hline $\begin{array}{l}\text { Cuscuta epithymum (L.) L. ssp. } \\
\text { epithymum }\end{array}$ & Cuscutaceae & $\mathrm{T}$ & WISP & & 15 & V\&al-2019 \\
\hline Cyclamen hederifolium Aiton & Primulaceae & G & SEU-me & & $13,18,19,37$ & V\&al-2019 \\
\hline $\begin{array}{l}\text { Cymbalaria muralis P. Gaertn., B. } \\
\text { Mey. et Scherb. ssp. visianii D. A. } \\
\text { Webb }\end{array}$ & Scrophulariaceae & $\mathrm{T}$ & ME-ilape & $\begin{array}{l}\text { end, } \\
\text { sp }\end{array}$ & $\begin{array}{l}\text { on the rocks of the eastern slopes of } \\
\text { Svilaja }\end{array}$ & $\begin{array}{l}\text { L\&al-1987 as C. pallida } \\
\text { (Ten.) }\end{array}$ \\
\hline Cynodon dactylon (L.) Pers. & Poaceae & G & WISP & & $\begin{array}{c}1,4,5,11,13,14,17,22,23,24,28,37 \\
42,43,48,68,69\end{array}$ & \\
\hline Cynoglossum columnae Ten & Boraginaceae & $\mathrm{T}$ & ME-east & & $1,2,20,30,49$ & V\&al-2019 \\
\hline Cynoglossum creticum Mill. & Boraginaceae & $\mathrm{T}$ & ME-circ & & $1,17,43,63$ & \\
\hline Cynosurus cristatus L. & Poaceae & $\mathrm{H}$ & WISP & & 21 & \\
\hline Cynosurus echinatus L. & Poaceae & $\mathrm{T}$ & SEU-me & & $5,45,48,54$ & \\
\hline Cyperus longus L. & Cyperaceae & Hy & EUAS & $\begin{array}{l}\mathrm{VU}, \\
\mathrm{sp}\end{array}$ & 22 & \\
\hline Cystopteris fragilis (L.) Bernh. & Woodsiaceae & $\mathrm{H}$ & WISP & & $10,11,32$ & \\
\hline $\begin{array}{l}\text { Cytisus procumbens (Willd.) } \\
\text { Spreng. }\end{array}$ & Fabaceae & $\mathrm{Ch}$ & SEU-mo & & 9 & \\
\hline Cytisus villosus Pourr. & Fabaceae & $\mathrm{Ch}$ & SEU-po & & 6,36 & \\
\hline $\begin{array}{l}\text { Dactylis glomerata L. ssp. } \\
\text { glomerata }\end{array}$ & Poaceae & $\mathrm{H}$ & EUAS & & $\begin{array}{c}1,4,8,13,14,16,19,20,21,22,32,34 \\
37,45,46,59,63,65,69\end{array}$ & $\begin{array}{l}\text { V\&al-2019 as D. } \\
\text { glomerata L. }\end{array}$ \\
\hline $\begin{array}{l}\text { Dactylis glomerata L. ssp. hispanica } \\
\text { (Roth) Nyman }\end{array}$ & Poaceae & $\mathrm{H}$ & ME-circ & & $\begin{array}{c}1,2,3,5,13,14,15,16,17,20,22,23 \\
25,26,27,29,37,38,42,44,45,47,50 \\
51,58,66,69,70\end{array}$ & \\
\hline Dactylorhiza maculata (L.) Soó & Orchidaceae & G & EUAS & $\mathrm{sp}$ & Svilaja, towards Orlove stine & Kr-2005:62 \\
\hline Dactylorhiza sambucina (L.) Soo & Orchidaceae & G & EURO & $\mathrm{sp}$ & $10,11,12,52,57,61$ & Kr-2005:70; V\&al-2019 \\
\hline Daphne alpina $\mathrm{L}$. & Thymelaeaceae & $\mathrm{P}$ & ME-po & & $9,30,33,34,35,40,51,52$ & $\begin{array}{l}\text { LRS-1989 as D. alpina s. } \\
\text { ampl. }\end{array}$ \\
\hline Daphne mezereum 1. & Thymelaeaceae & $\mathrm{P}$ & EUAS & NT & 36 & \\
\hline $\begin{array}{l}\text { Dasypyrum villosum (L.) P. } \\
\text { Candargy }\end{array}$ & Poaceae & $\mathrm{T}$ & ME-po & & $\begin{array}{c}1,3,5,14,16,20,21,23,24,25,28,42 \\
43,46,49\end{array}$ & M\&K-2016 \\
\hline Datura wrightii Regel & Solanaceae & $\mathrm{T}$ & CUAD & inv & 1 & \\
\hline Datura stramonium L. & Solanaceae & $\mathrm{T}$ & WISP & & $3,5,14,17,66,68$ & \\
\hline Daucus carota L. ssp. carota & Apiaceae & $\mathrm{H}$ & WISP & & $13,15,50,66$ & $\begin{array}{l}\text { V\&al-2019, as D. carota } \\
\text { L. }\end{array}$ \\
\hline $\begin{array}{l}\text { Daucus carota L. ssp. major (Vis.) } \\
\text { Arcang. }\end{array}$ & Apiaceae & $\mathrm{T}$ & ME-ilade & & $1,3,4,22,25,46,59$ & \\
\hline $\begin{array}{l}\text { Daucus carota L. ssp. maritimus } \\
\text { (Lam.) Batt. }\end{array}$ & Apiaceae & $\mathrm{T}$ & ME-west & & 4,22 & \\
\hline $\begin{array}{l}\text { Delphinium fissum Waldst. et Kit. } \\
\text { ssp. fissum }\end{array}$ & Ranunculaceae & $\mathrm{H}$ & SEU-po & & 41 & \\
\hline Delphinium peregrinum L. & Ranunculaceae & $\mathrm{T}$ & SEU-me & $\mathrm{EN}, \mathrm{sp}$ & $1,4,43$ & V\&al-2019 \\
\hline $\begin{array}{l}\text { Deschampsia media (Gouan) } \\
\text { Roem. et Schult. }\end{array}$ & Poaceae & $\mathrm{H}$ & SEU-atl & & "ad pedem montis Svilaja" & $\begin{array}{l}\text { Vi-1842:67 as Aira media } \\
\text { Gouan }\end{array}$ \\
\hline
\end{tabular}




\begin{tabular}{|c|c|c|c|c|c|c|}
\hline Taxon & Family & $\begin{array}{c}\text { Life } \\
\text { form }\end{array}$ & Geoelement & Status & Localities (finding sites) & $\begin{array}{c}\text { Previously recorded } \\
\text { findings }\end{array}$ \\
\hline Desmazeria rigida (L.) Tutin & Poaceae & $\mathrm{T}$ & ME-atl & & $1,5,14,18,19,20,21,22,25,26,27,37$ & \\
\hline Dianthus armeria $\mathrm{L}$. & Caryophyllaceae & $\mathrm{T}$ & EURO & $\mathrm{sp}$ & Significant Landscape of Sutina & V\&al-2019 \\
\hline $\begin{array}{l}\text { Dianthus carthusianorum L. ssp. } \\
\text { sanguineus (Vis.) Hegi }\end{array}$ & Caryophyllaceae & $\mathrm{H}$ & ME-ilade & $\mathrm{sp}$ & 55 & \\
\hline $\begin{array}{l}\begin{array}{l}\text { Dianthus ciliatus Guss. ssp. } \\
\text { ciliatus }\end{array} \\
\end{array}$ & Caryophyllaceae & $\mathrm{H}$ & ME-ilade & $\begin{array}{c}\text { end, } \\
\text { sp }\end{array}$ & 65 & \\
\hline $\begin{array}{l}\text { Dianthus ferrugineus Mill. ssp. } \\
\text { liburnicus (Bartl.) Tutin }\end{array}$ & Caryophyllaceae & $\mathrm{H}$ & ME-ilade & $\begin{array}{l}\text { end, } \\
\text { sp }\end{array}$ & $5,11,25,39,40,41,65$ & V\&al-2019 \\
\hline $\begin{array}{l}\text { Dianthus sylvestris Wulfen in } \\
\text { Jacq. ssp. nodosus (Tausch) } \\
\text { Hayek }\end{array}$ & Caryophyllaceae & $\mathrm{H}$ & ME-ilade & $\begin{array}{c}\text { end, } \\
\text { sp }\end{array}$ & 56,58 & \\
\hline $\begin{array}{l}\text { Dianthus sylvestris Wulfen in } \\
\text { Jacq. ssp. sylvestris }\end{array}$ & Caryophyllaceae & $\mathrm{H}$ & SEU-mo & $\mathrm{sp}$ & $9,11,50,51,53,57,65$ & \\
\hline $\begin{array}{l}\text { Dianthus sylvestris Wulfen in } \\
\text { Jacq. ssp. tergestinus (Reichenb.) } \\
\text { Hayek }\end{array}$ & Caryophyllaceae & $\mathrm{H}$ & ME-ilade & $\begin{array}{l}\text { end, } \\
\text { sp }\end{array}$ & $1,6,8,9,23,31,33,58$ & $\begin{array}{l}\text { Tr-1992 as D. tergestinus } \\
\text { (Rchb.) Kern.; } \\
\text { VRZ-2012; V\&al-2019 }\end{array}$ \\
\hline $\begin{array}{l}\text { Dichanthium ischaemum (L.) } \\
\text { Roberty }\end{array}$ & Poaceae & $\mathrm{H}$ & SEU-me & & $\begin{array}{c}6,13,15,17,18,19,21,22,23,25,26 \\
27,43,48,49,68\end{array}$ & \\
\hline Dictamnus albus L. & Rutaceae & $\mathrm{Ch}$ & EUAS & & $13,14,20,25$ & \\
\hline Digitalis grandiflora Mill. & Scrophulariaceae & $\mathrm{H}$ & SEU-po & NT & 53,65 & \\
\hline Digitalis laevigata Waldst. et Kit. & Scrophulariaceae & $\mathrm{H}$ & ILBA-end & & $9,29,31$ & \\
\hline $\begin{array}{l}\text { Digitaria ischaemum (Schreb.) } \\
\text { Muhl. }\end{array}$ & Poaceae & $\mathrm{T}$ & WISP & $\begin{array}{c}\text { DD, } \\
\text { sp }\end{array}$ & Significant Landscape of Sutina & V\&al-2019 \\
\hline Digitaria sanguinalis (L.) Scop. & Poaceae & $\mathrm{T}$ & WISP & & $4,14,65$ & \\
\hline Diplotaxis muralis (L.) DC. & Brassicaceae & $\mathrm{T}$ & WISP & & $16,22,49,50,66$ & V\&al-2019 \\
\hline Diplotaxis tenuifolia (L.) DC. & Brassicaceae & $\mathrm{H}$ & WISP & & $\begin{array}{c}1,3,4,6,13,14,16,22,27,28,43,46 \\
69,70\end{array}$ & V\&al-2019 \\
\hline Diplotaxis viminea (L.) DC. & Brassicaceae & $\mathrm{T}$ & ME-circ & & 13,27 & \\
\hline Dipsacus fullonum L. & Dipsacaceae & $\mathrm{H}$ & WISP & & $3,4,13,22,23,25,44,46$ & Ma-1992 \\
\hline Dipsacus laciniatus L. & Dipsacaceae & $\mathrm{H}$ & EUAS & & $3,22,59$ & Vi-1847:11 \\
\hline Dittrichia graveolens (L.) Greuter & Asteraceae & $\mathrm{T}$ & SEU-me & & 68 & \\
\hline Dittrichia viscosa (L.) Greuter & Asteraceae & $\mathrm{H}$ & ME-circ & & Significant Landscape of Sutina & V\&al-2019 \\
\hline $\begin{array}{l}\text { Dorycnium germanicum (Gremli) } \\
\text { Rikli }\end{array}$ & Fabaceae & $\mathrm{H}$ & SEU-me & & $\begin{array}{c}8,9,11,26,30,31,32,33,35,36,38 \\
39,40,41,45,50,52,53,54,56,59,62 \\
65,69\end{array}$ & V\&al-2019 \\
\hline Dorycnium herbaceum Vill & Fabaceae & $\mathrm{H}$ & SEU-me & & $1,7,13,18,19,22,23,28,46$ & \\
\hline Dorycnium hirsutum (L.) Ser. & Fabaceae & $\mathrm{Ch}$ & ME-circ & & 26 & \\
\hline Draba lasiocarpa Rochel & Brassicaceae & $\mathrm{H}$ & SEEU & & Svilaja mountain range & L\&R-1989 \\
\hline Dryopteris filix-mas (L.) Schott & Dryopteridaceae & G & WISP & & $21,26,32$ & \\
\hline $\begin{array}{l}\text { Dryopteris villarii (Bellardi) } \\
\text { Woynar ex Schinz et Thell. }\end{array}$ & Dryopteridaceae & $\mathrm{H}$ & ME-circ & & 32 & $\begin{array}{l}\text { Vi-1842:37 as Aspidium } \\
\text { rigidum Sw.; V\&al-2019 }\end{array}$ \\
\hline Ecballium elaterium (L.) A.Rich. & Cucurbitaceae & $\mathrm{Ch}$ & ME-circ & DD & 1,68 & \\
\hline $\begin{array}{l}\text { Echinochloa crus-galli (L.) P. } \\
\text { Beauv. }\end{array}$ & Poaceae & $\mathrm{T}$ & WISP & & $4,14,22,28,48,68$ & \\
\hline $\begin{array}{l}\text { Echinops ritro L. ssp. ruthenicus } \\
\text { (M. Bieb.) Nyman }\end{array}$ & Asteraceae & $\mathrm{H}$ & SEU-po & DD & $31,33,34,44,47$ & V\&al-2019 as E. ritro L. \\
\hline Echium italicum $\mathrm{L}$. & Boraginaceae & $\mathrm{H}$ & ME-circ & & $\begin{array}{c}3,4,5,13,20,22,23,25,27,28,43,44 \\
45,46,47,60,61\end{array}$ & V\&al-2019 \\
\hline Echium plantagineum $\mathrm{L}$. & Boraginaceae & $\mathrm{T}$ & ME-atl & & 1 & \\
\hline Echium vulgare $\mathrm{L}$. & Boraginaceae & $\mathrm{H}$ & EURO & & $\begin{array}{c}8,9,11,13,15,17,20,21,25,27,31 \\
32,34,35,38,40,43,46,48,50,53,55 \\
59,65,66,69\end{array}$ & V\&al-2019 \\
\hline $\begin{array}{l}\text { Edraianthus dalmaticus (A.DC.) } \\
\text { A.DC. }\end{array}$ & Campanulaceae & $\mathrm{H}$ & ME-ilade & $\begin{array}{l}\text { end, } \\
\text { DD, } \\
\text { sp }\end{array}$ & near Vrlika and Muć & $\begin{array}{l}\text { L\&al-1987, as E. d. var. } \\
\text { dolomiticus Lak. }\end{array}$ \\
\hline $\begin{array}{l}\text { Edraianthus graminifolius (L.) } \\
\text { A.DC. ssp. graminifolius }\end{array}$ & Campanulaceae & $\mathrm{Ch}$ & ILBA-end & & 33,34 & $\begin{array}{l}\text { Ja-1908; LRS-1989; } \\
\text { L\&R-1989 as E. caricinus } \\
\text { Schott (s. str.) }\end{array}$ \\
\hline $\begin{array}{l}\text { Edraianthus tenuifolius (Waldst. et } \\
\text { Kit.) A.DC. }\end{array}$ & Campanulaceae & $\mathrm{Ch}$ & ME-ilade & $\begin{array}{c}\text { end, } \\
\text { sp }\end{array}$ & $\begin{array}{c}8,9,10,31,32,33,34,38,39,40,41 \\
51,52,53,54,55,67,69\end{array}$ & $\begin{array}{l}\text { Ja-1908; L\&al-1987 as E. } \\
\text { t. var. dolomiticus Lak.; } \\
\text { VRZ-2012; Se\&al-2016; } \\
\text { V\&al-2019 }\end{array}$ \\
\hline
\end{tabular}




\begin{tabular}{|c|c|c|c|c|c|c|}
\hline Taxon & Family & $\begin{array}{c}\text { Life } \\
\text { form }\end{array}$ & Geoelement & Status & Localities (finding sites) & $\begin{array}{l}\text { Previously recorded } \\
\text { findings }\end{array}$ \\
\hline $\begin{array}{l}\text { Eleocharis palustris (L.) Roem. et } \\
\text { Schult. }\end{array}$ & Cyperaceae & G & WISP & & $17,23,28$ & $\begin{array}{l}\text { L\&R-1989, as Heleocharis } \\
\text { uniglumis }\end{array}$ \\
\hline Elymus caninus (L.) L. & Poaceae & $\mathrm{H}$ & $\mathrm{CIHO}$ & & $\begin{array}{l}\text { "ad sepes et in dumetis circa } \\
\text { Verlika" }\end{array}$ & $\begin{array}{l}\text { Vi-1842:94 as Triticum } \\
\text { caninum L. }\end{array}$ \\
\hline Elymus hispidus (Opiz) Melderis & Poaceae & G & SEU-me & & $22,31,38$ & \\
\hline Elymus repens (L.) Gould & Poaceae & G & WISP & & $\begin{array}{c}1,11,13,15,17,21,22,23,25,35,43 \\
46,50,58,65,70\end{array}$ & V\&al-2019 \\
\hline $\begin{array}{l}\text { Epilobium angustifolium } \mathrm{L} . \text { (= } \\
\text { Chamamerion angustifolium) }\end{array}$ & Onagraceae & $\mathrm{H}$ & WISP & & $10,11,32,34,35,38,39,40,55,65$ & V\&al-2019 \\
\hline Epilobium hirsutum L. & Onagraceae & $\mathrm{H}$ & EUAS & & 14 & V\&al-2019 \\
\hline Epilobium montanum L. & Onagraceae & $\mathrm{H}$ & EUAS & & 35 & \\
\hline $\begin{array}{l}\text { Epipactis artrorubens (Hoffm.) } \\
\text { Besser }\end{array}$ & Orchidaceae & G & EUAS & $\mathrm{sp}$ & Svilaja, Orlove stine & Kr-2005:72 \\
\hline $\begin{array}{l}\text { Epipactis greuteri } \mathrm{H} \text {. Baumann et } \\
\text { Künkele }\end{array}$ & Orchidaceae & G & ME-eu & $\mathrm{sp}$ & $\begin{array}{c}\text { site } 263 \text { (XJ2345) SO Zelovo, 820-840 } \\
\text { m }\end{array}$ & Df-2006 \\
\hline Epipactis helleborine (L.) Crantz & Orchidaceae & G & EUAS & $\mathrm{sp}$ & 17 & Kr-2005:78; V\&al-2019 \\
\hline Epipactis microphylla (Ehrh.) Sw. & Orchidaceae & G & EUAS & $\mathrm{sp}$ & 62 & \\
\hline Epipactis muelleri Godfery & Orchidaceae & G & SEU-me & $\mathrm{sp}$ & $\begin{array}{c}\text { site } 263 \text { (XJ2345) SO Zelovo, 820-840 } \\
\mathrm{m} \\
\end{array}$ & Df-2006 \\
\hline $\begin{array}{l}\text { Epipactis placentina } \\
\text { Bongiorni \& Grünanger }\end{array}$ & Orchidaceae & G & ME-eu & $\mathrm{sp}$ & $58(x=4844918, y=6382266)$ & Df-2006 \\
\hline Equisetum arvense L. & Equisetaceae & $\mathrm{G}$ & $\mathrm{CIHO}$ & & $14,22,64,69,70$ & V\&al-2019 \\
\hline Equisetum hyemale L. & Equisetaceae & G & $\mathrm{CIHO}$ & $\mathrm{sp}$ & Significant Landscape of Sutina & V\&al-2019 \\
\hline Equisetum palustre L. & Equisetaceae & $\mathrm{G}$ & $\mathrm{CIHO}$ & & $14,46,69$ & V\&al-2019 \\
\hline $\begin{array}{l}\text { Equisetum variegatum } \mathrm{F} \text {. Weber et } \\
\text { D. Mohr }\end{array}$ & Equisetaceae & G & $\mathrm{CIHO}$ & DD & 69,70 & \\
\hline $\begin{array}{l}\text { Eragrostis cilianensis (All.) } \\
\text { F.T.Hubb. }\end{array}$ & Poaceae & $\mathrm{T}$ & WISP & & $3,4,14,22,68$ & \\
\hline Eragrostis minor Host & Poaceae & $\mathrm{T}$ & ME-circ & & $14,26,27$ & \\
\hline $\begin{array}{l}\text { Erigeron annuus (L.) Pers. ssp. } \\
\text { annuus }\end{array}$ & Asteraceae & $\mathrm{T}$ & CUAD & inv & $\begin{array}{c}1,3,4,13,14,15,22,23,27,28,40,51 \\
58,65,69\end{array}$ & $\begin{array}{l}\text { V\&al-2019 as E. annuus } \\
\text { (L.) Pers. }\end{array}$ \\
\hline $\begin{array}{l}\text { Erigeron annuus (L.) Pers. ssp. } \\
\text { septentrionalis (Fernald et } \\
\text { Wiegand) Wagenit }\end{array}$ & Asteraceae & $\mathrm{T}$ & CUAD & inv & $\begin{array}{c}1,4,13,14,15,16,17,22,25,28,31 \\
41,42,43,53,59,65,66\end{array}$ & \\
\hline $\begin{array}{l}\text { Erigeron annuus (L.) Pers. ssp. } \\
\text { strigosus (Mühlenb. ex Willd.) } \\
\text { Wagenitz }\end{array}$ & Asteraceae & $\mathrm{T}$ & CUAD & inv & 18 & \\
\hline $\begin{array}{l}\text { Erodium acaule (L.) Becherer et } \\
\text { Thell. }\end{array}$ & Geraniaceae & $\mathrm{H}$ & SEU-mo & & $7,8,11,13,20,43$ & \\
\hline Erodium ciconium (L.) L' Hér. & Geraniaceae & $\mathrm{T}$ & ME-po & & Significant Landscape of Sutina & V\&al-2019 \\
\hline Erodium cicutarium (L.) L’ Hér & Geraniaceae & $\mathrm{T}$ & WISP & & $\begin{array}{c}5,6,11,13,14,16,20,24,26,27,29 \\
47,49,50,65,66,70\end{array}$ & V\&al-2019 \\
\hline Erodium malacoides (L.) L’Hér. & Geraniaceae & $\mathrm{T}$ & ME-circ & & 27 & \\
\hline $\begin{array}{l}\text { Erophila verna (L.) Chevall. ssp. } \\
\text { praecox (Steven) Walters }\end{array}$ & Brassicaceae & $\mathrm{T}$ & ME-circ & & $2,10,11,12,29,66$ & \\
\hline $\begin{array}{l}\text { Erophila verna (L.) Chevall. ssp. } \\
\text { verna }\end{array}$ & Brassicaceae & $\mathrm{T}$ & WISP & & Significant Landscape of Sutina & V\&al-2019 \\
\hline $\begin{array}{l}\text { Eruca vesicaria (L.) Cav. ssp. sativa } \\
\text { (Mill.) Thell. }\end{array}$ & Brassicaceae & $\mathrm{T}$ & CUAD & & 37 & \\
\hline Eryngium amethystinum L. & Apiaceae & $\mathrm{H}$ & ME-ilseu & & $\begin{array}{c}2,4,5,6,7,8,13,15,16,17,18,19,20 \\
22,23,25,26,28,29,31,37,39,40,41 \\
42,43,44,45,46,47,48,49,51,52,53 \\
54,55,56,57,58,59,60,61,62,65,66 \\
67,69,70\end{array}$ & $\begin{array}{l}\text { Je-1952; Tr-1992; } \\
\text { V\&al-2019 }\end{array}$ \\
\hline Eryngium campestre L. & Apiaceae & $\mathrm{H}$ & SEU-me & & 1 & V\&al-2019 \\
\hline $\begin{array}{l}\text { Erysimum sylvestre (Crantz) Scop. } \\
\text { ssp. sylvestre }\end{array}$ & Brassicaceae & $\mathrm{H}$ & ME-ilade & $\begin{array}{l}\text { end, } \\
\text { sp }\end{array}$ & 38,39 & \\
\hline Erythronium dens-canis L. & Liliaceae & G & SEU-co & & 36,67 & Mi\&al-2013; V\&al-2019 \\
\hline Euonymus europaeus L & Celastraceae & $\mathrm{P}$ & EUAS & & $\begin{array}{c}4,13,14,15,24,25,27,28,30,32,40 \\
44,45,46,47,48,51,52,58,60,65,66 \\
67,70 \\
\end{array}$ & \\
\hline Euonymus verrucosa Scop. & Celastraceae & $\mathrm{P}$ & EEUPO & & $36,44,50,52,65$ & V\&al-2019 \\
\hline
\end{tabular}




\begin{tabular}{|c|c|c|c|c|c|c|}
\hline Taxon & Family & \begin{tabular}{|c|} 
Life \\
form
\end{tabular} & Geoelement & Status & Localities (finding sites) & $\begin{array}{c}\text { Previously recorded } \\
\text { findings }\end{array}$ \\
\hline $\begin{array}{l}\text { Eupatorium cannabinum L. ssp. } \\
\text { cannabinum }\end{array}$ & Asteraceae & $\mathrm{H}$ & ME-east & & $14,22,46,69,70$ & $\begin{array}{l}\text { V\&al-2019 as E. } \\
\text { cannabinum L. }\end{array}$ \\
\hline Euphorbia chamaesyce L. & Euphorbiaceae & $\mathrm{T}$ & SEU-me & & 22,27 & \\
\hline Euphorbia cyparissias L. & Euphorbiaceae & $\mathrm{H}$ & EUAS & & $6,12,30,31,40,58$ & V\&al-2019 \\
\hline Euphorbia falcata L. & Euphorbiaceae & $\mathrm{T}$ & SEU-me & & $1,23,25,26,27,43,45,59,62$ & V\&al-2019 \\
\hline Euphorbia fragifera Jan & Euphorbiaceae & $\mathrm{Ch}$ & ME-ilade & & $1,6,30$ & \\
\hline Euphorbia helioscopia L. & Euphorbiaceae & $\mathrm{T}$ & WISP & & $\begin{array}{c}2,11,17,27,29,30,37,43,45,47,50 \\
60,66,70\end{array}$ & Ma-1992 \\
\hline Euphorbia nicaeensis All. & Euphorbiaceae & G & EUAS & & Significant Landscape of Sutina & V\&al-2019 \\
\hline Euphorbia peplus L. & Euphorbiaceae & $\mathrm{T}$ & WISP & & 14 & \\
\hline Euphorbia prostrata Aiton & Euphorbiaceae & $\mathrm{T}$ & CUAD & $\begin{array}{l}\mathrm{LC}, \\
\text { inv }\end{array}$ & $3,22,27$ & \\
\hline Euphorbia spinosa L. & Euphorbiaceae & $\mathrm{Ch}$ & ME-circ & & $1,2,6,23,25,27,31,47$ & \\
\hline $\begin{array}{l}\text { Euphorbia barrelieri Savi ssp. } \\
\text { hercegovina (Beck) Kuzmanov }\end{array}$ & Euphorbiaceae & $\mathrm{Ch}$ & ME-ilade & & 69 & \\
\hline Euphrasia liburnica Wettst. & Scrophulariaceae & $\mathrm{T}$ & ILBA-end & & Significant Landscape of Sutina & V\&al-2019 \\
\hline Euphrasia salisburgensis Funck & Scrophulariaceae & $\mathrm{T}$ & SEU-mo & & 50,59 & \\
\hline $\begin{array}{l}\text { Euphrasia stricta Wolff ex J. F. } \\
\text { Lehm. }\end{array}$ & Scrophulariaceae & $\mathrm{T}$ & CEU & & 46 & \\
\hline Fagus sylvatica L. & Fagaceae & $\mathrm{P}$ & EURO & & $\begin{array}{c}9,10,11,12,32,34,35,36,38,39,40 \\
55,57,58,63,64\end{array}$ & $\begin{array}{l}\text { Ja-1908; Je-1952; } \\
\text { L\&al-1987 as F. moesiaca } \\
\text { (Maly) Czec.; V\&al-2019 }\end{array}$ \\
\hline $\begin{array}{l}\text { Fallopia baldschuanica (Regel) } \\
\text { Holub }\end{array}$ & Polygonaceae & $\mathrm{P}$ & CUAD & & 1,27 & \\
\hline Fallopia convolvulus (L.) Á.Löve & Polygonaceae & $\mathrm{T}$ & $\mathrm{CIHO}$ & & $\begin{array}{c}1,3,4,5,11,13,14,15,16,20,24,27 \\
28,29,37,40,43,50\end{array}$ & \\
\hline Ferulago sylvatica (Besser) Rchb. & Apiaceae & $\mathrm{H}$ & EEUPO & & Significant Landscape of Sutina & V\&al-2019 \\
\hline Festuca arundinacea Schreb. & Poaceae & $\mathrm{H}$ & EUAS & & $14,28,42$ & \\
\hline Festuca dalmatica (Hack.) K. Richt. & Poaceae & $\mathrm{H}$ & ILBA-end & & 15,36 & \\
\hline Festuca heterophylla Lam. & Poaceae & $\mathrm{H}$ & EUAS & & 31 & \\
\hline Festuca illyrica Markgr.-Dann. & Poaceae & $\mathrm{H}$ & ME-ilade & $\begin{array}{c}\text { end, } \\
\text { sp }\end{array}$ & $\begin{array}{c}\text { Svilaja mountain range; above the } \\
\text { village of Muć }\end{array}$ & $\begin{array}{l}\text { L\&al-1987; LRS-1989; } \\
\text { Tr-1992 }\end{array}$ \\
\hline Festuca ovina L. & Poaceae & $\mathrm{H}$ & CEU & & Significant Landscape of Sutina & V\&al-2019 \\
\hline $\begin{array}{l}\text { Festuca panciciana (Hack.) K. } \\
\text { Richt. }\end{array}$ & Poaceae & $\mathrm{H}$ & ILBA-end & & Svilaja mountain range & LRS-1989 \\
\hline Festuca pratensis Huds. & Poaceae & $\mathrm{H}$ & WISP & & $\begin{array}{c}1,9,11,14,16,17,20,23,28,42,46 \\
50,51,53,55,65,68,69\end{array}$ & \\
\hline Festuca rubra L. & Poaceae & $\mathrm{H}$ & $\mathrm{CIHO}$ & & Svilaja mountain range & L\&R-1989 \\
\hline Festuca rupicola Heuff. & Poaceae & $\mathrm{H}$ & SEEU & & 1,9 & LRS-1989 \\
\hline $\begin{array}{l}\text { Festuca trachyphylla (Hack.) } \\
\text { Krajina }\end{array}$ & Poaceae & $\mathrm{H}$ & CEU & & Svilaja mountain range & LRS-1989 \\
\hline $\begin{array}{l}\text { Festuca valesiaca Schleich. ex } \\
\text { Gaudin }\end{array}$ & Poaceae & $\mathrm{H}$ & SEU-po & & $5,11,16,20,29,44,48,49,51,52$ & $\begin{array}{l}\text { Je-1952; LRS-1989; } \\
\text { V\&al-2019 }\end{array}$ \\
\hline Ficus carica $\mathrm{L}$. & Moraceae & $P$ & ME-circ & & $\begin{array}{c}1,3,4,14,16,20,21,22,23,25,27,28 \\
30,37,42,43,45,46,47,69\end{array}$ & M\&K-2016; V\&al-2019 \\
\hline Filago pyramidata L. & Asteraceae & $\mathrm{T}$ & ME-atl & & $17,21,25,26,31$ & \\
\hline Filago vulgaris Lam. & Asteraceae & $\mathrm{T}$ & EUAS & & $5,7,13$ & \\
\hline Filipendula vulgaris Moench & Rosaceae & $\mathrm{H}$ & EUAS & & $\begin{array}{c}2,6,11,12,13,17,18,19,20,25,26 \\
27,29,30,32,39,40,41,43,48,51,52 \\
53,55,57,58,59,61,65,67\end{array}$ & V\&al-2019 \\
\hline Foeniculum vulgare Mill. & Apiaceae & $\mathrm{H}$ & ME-circ & & $17,22,28,29,43$ & V\&al-2019 \\
\hline Fragaria vesca $\mathrm{L}$ & Rosaceae & $\mathrm{H}$ & WISP & & $\begin{array}{c}2,11,14,18,20,22,23,25,29,37,38 \\
39,40,41,42,63,64,65\end{array}$ & Pe:1832:37; V\&al-2019 \\
\hline Frangula alnus Mill. & Rhamnaceae & $\mathrm{P}$ & CEU & & Significant Landscape of Sutina & V\&al-2019 \\
\hline Frangula rupestris (Scop.) Schur. & Rhamnaceae & $\mathrm{P}$ & ME-ilseu & & $\begin{array}{l}1,2,9,17,25,26,27,30,31,33,34,35 \\
37,41,42,44,45,48,56,61,65,69,70\end{array}$ & $\begin{array}{l}\text { Ja-1908 as Rhamnus } \\
\text { rupestris; L\&al-1987; } \\
\text { V\&al-2019 }\end{array}$ \\
\hline $\begin{array}{l}\text { Fraxinus angustifolia Vahl ssp. } \\
\text { oxycarpa (M. Bieb.ex Willd.) } \\
\text { Francoet Rocha Afonso }\end{array}$ & Oleaceae & $P$ & ME-circ & & $16,22,46$ & $\begin{array}{l}\text { L\&al-1987 as F. } \\
\text { angustifolia Vahl. }\end{array}$ \\
\hline Fraxinus excelsior $\mathrm{L}$. & Oleaceae & $\mathrm{P}$ & EURO & & 32 & Vi-1852:22 \\
\hline
\end{tabular}




\begin{tabular}{|c|c|c|c|c|c|c|}
\hline Taxon & Family & \begin{tabular}{|c|} 
Life \\
form
\end{tabular} & Geoelement & Status & Localities (finding sites) & $\begin{array}{l}\text { Previously recorded } \\
\text { findings }\end{array}$ \\
\hline Fraxinus ornus L. & Oleaceae & $P$ & SEU-me & & $\begin{array}{c}1,2,4,5,6,7,9,11,13,14,15,16,17, \\
18,19,20,21,22,23,25,26,27,28,29, \\
30,31,32,33,34,35,36,37,38,39,40, \\
41,42,43,44,45,46,47,48,49,50,51, \\
52,53,55,56,57,58,59,61,62,63,64, \\
65,66,67,69,70\end{array}$ & $\begin{array}{l}\text { Ja-1908; Je-1952; } \\
\text { Me-1972; L\&al-1987; } \\
\text { V\&al-2019 }\end{array}$ \\
\hline $\begin{array}{l}\text { Fritillaria messanensis Raf. ssp. } \\
\text { neglecta (Parl.) Nyman }\end{array}$ & Liliaceae & G & ILBA-end & end & 40,41 & K\&S-2016; V\&al-2019 \\
\hline $\begin{array}{l}\text { Fritillaria montana Hoppe ex } \\
\text { W.D.J.Koch }\end{array}$ & Liliaceae & G & SEU-mo & & $10,12,32,41$ & $\begin{array}{l}\text { Vi-1842:131; L\&R-1989; } \\
\text { Mi\&al-2013 as F. } \\
\text { orientalis Adams; } \\
\text { K\&S-2016 }\end{array}$ \\
\hline Fumana ericifolia Wallr. & Cistaceae & $\mathrm{Ch}$ & ME-west & & $26,41,52,54,55,56,58$ & \\
\hline $\begin{array}{l}\text { Fumana procumbens (Dunal) } \\
\text { Gren. et Godr. }\end{array}$ & Cistaceae & $\mathrm{Ch}$ & SEU-me & & $8,23,31,43,45,50,53,59,70$ & $\begin{array}{l}\text { Tr-1992 as F. vulgaris } \\
\text { Spach.; VRZ-2012; } \\
\text { Se\&al-2016; V\&al-2019 }\end{array}$ \\
\hline Fumaria officinalis L. & Fumariaceae & $\mathrm{T}$ & WISP & & $16,24,37,43,68,70$ & \\
\hline Gagea lutea (L.) Ker Gawl. & Liliaceae & G & EUAS & & 41 & V\&al-2019 \\
\hline $\begin{array}{l}\text { Gagea pusilla (F. W. Schmidt) } \\
\text { Sweet }\end{array}$ & Liliaceae & G & ME-po & & Significant Landscape of Sutina & V\&al-2019 \\
\hline Gaillardia aristata Pursh.* & Asteraceae & $\mathrm{H}$ & CUAD & & $3 * 4$ & \\
\hline Galeopsis angustifolia Hoffm. & Lamiaceae & $\mathrm{T}$ & EUAS & & 55 & \\
\hline Galinsoga parviflora Cav. & Asteraceae & $\mathrm{T}$ & CUAD & inv & $13,14,16,65,66,68$ & \\
\hline Galium album Mill. & Rubiaceae & $\mathrm{H}$ & EUAS & & 13 & Ma-1964 \\
\hline Galium aparine $\mathrm{L}$. & Rubiaceae & $\mathrm{T}$ & WISP & & $\begin{array}{l}1,2,3,14,16,17,20,23,25,27,29,37 \\
42,43,44,45,46,47,49,50,60,61,69\end{array}$ & Ma-1992; V\&al-2019 \\
\hline Galium corrudifolium Vill. & Rubiaceae & $\mathrm{H}$ & SEU-me & & $\begin{array}{c}1,6,7,15,17,19,21,23,25,29,31,42 \\
45,47,50,53,59,61,63,66,69\end{array}$ & $\begin{array}{l}\text { Tr-1992; VRZ-2012; } \\
\text { V\&al-2019 }\end{array}$ \\
\hline Galium firmum Tausch & Rubiaceae & $\mathrm{H}$ & ME-eu & $\begin{array}{l}\text { end, } \\
\text { sp }\end{array}$ & 34,35 & LRS-1989 \\
\hline Galium lucidum All. & Rubiaceae & $\mathrm{H}$ & SEU-me & & $9,10,11,14,33,43,63$ & M\&K-2016; LRS-1989 \\
\hline Galium mollugo L. & Rubiaceae & $\mathrm{H}$ & EUAS & & $\begin{array}{c}14,15,16,28,37,46,47,60,63,64,69 \\
70\end{array}$ & Ma-1992; V\&al-2019 \\
\hline Galium odoratum (L.) Scop. & Rubiaceae & G & EUAS & & 35 & \\
\hline Galium palustre L. & Rubiaceae & $\mathrm{H}$ & WISP & & 22,28 & \\
\hline Galium sylvaticum L. & Rubiaceae & G & EURO & & 32 & \\
\hline Galium verum $\mathrm{L}$. & Rubiaceae & $\mathrm{H}$ & WISP & & $\begin{array}{c}1,2,4,7,8,11,13,15,16,18,19,21 \\
22,23,25,28,37,38,39,40,41,42,43 \\
44,46,51,53,54,55,58,59,60,62,65 \\
66,69,70\end{array}$ & V\&al-2019 \\
\hline Genista januensis Viv. & Fabaceae & $\mathrm{Ch}$ & ILBA-bap & & $2,10,11,13,14,26$ & \\
\hline Genista pulchella Vis. ssp. pulchella & Fabaceae & $\mathrm{Ch}$ & ILBA-end & $\begin{array}{l}\text { end, } \\
\text { sp }\end{array}$ & Svilaja mountain range & LRS-1989 \\
\hline Genista radiata (L.) Scop. & Fabaceae & $\mathrm{Ch}$ & SEU-mo & & 34 & \\
\hline Genista sericea Wulfen & Fabaceae & $\mathrm{Ch}$ & ME-east & $\begin{array}{l}\text { end, } \\
\text { sp }\end{array}$ & 11 & LRS-1989 \\
\hline Genista tinctoria $\mathrm{L}$. & Fabaceae & $\mathrm{Ch}$ & EUAS & & Significant Landscape of Sutina & V\&al-2019 \\
\hline $\begin{array}{l}\text { Genista sylvestris Scop. ssp. } \\
\text { dalmatica (Bartl.) H. Lindb. }\end{array}$ & Fabaceae & $\mathrm{Ch}$ & ME-ilade & $\begin{array}{l}\text { end, } \\
\text { sp }\end{array}$ & $1,17,23,26,31,51,57,61,65,67$ & $\begin{array}{l}\text { Tr-1992 as G. dalmatica } \\
\text { Bartl. et Wendl.; } \\
\text { VRZ-2012; V\&al-2019 }\end{array}$ \\
\hline $\begin{array}{l}\text { Genista sylvestris Scop. ssp. } \\
\text { sylvestris }\end{array}$ & Fabaceae & $\mathrm{Ch}$ & ME-ilade & & $\begin{array}{c}11,30,33,38,40,41,43,44,45,47,51 \\
52,53,56,58,62,64,69,70\end{array}$ & $\begin{array}{l}\text { LRS-1989 as G. sylvestris } \\
\text { Scop. ssp. innocua (Vis.) } \\
\text { Hay. }\end{array}$ \\
\hline Gentiana cruciata L. & Gentianaceae & $\mathrm{H}$ & EUAS & & 8,62 & Se-2016; V\&al-2019 \\
\hline Gentiana dinarica Beck & Gentianaceae & $\mathrm{H}$ & SEEU & NT & 41 & Se-2016 \\
\hline Gentiana tergestina Beck & Gentianaceae & $\mathrm{H}$ & ME-ilseu & & 34 & \\
\hline Gentiana utriculosa L. & Gentianaceae & $\mathrm{T}$ & CEU & & 52,53 & \\
\hline Gentiana verna $\mathrm{L}$. & Gentianaceae & $\mathrm{H}$ & EUAS & & 10,12 & L\&R-1989 \\
\hline Gentianella crispata (Vis.) Holub & Gentianaceae & $\mathrm{H}$ & ILBA-end & & Svilaja mountain range & LRS-1989 \\
\hline Geranium columbinum L. & Geraniaceae & $\mathrm{T}$ & EUAS & & $8,17,23,40,42,43,45,46,51,55,58$ & \\
\hline Geranium dissectum L. & Geraniaceae & $\mathrm{T}$ & WISP & & $2,3,16,17,28,37,44,45,47,60,69,70$ & V\&al-2019 \\
\hline Geranium lucidum L. & Geraniaceae & $\mathrm{T}$ & ME-atl & & $\begin{array}{c}2,10,11,12,13,14,16,19,20,26,27 \\
32,36,37,40,42,44,61,64\end{array}$ & Ja-1908; V\&al-2019 \\
\hline
\end{tabular}




\begin{tabular}{|c|c|c|c|c|c|c|}
\hline Taxon & Family & $\begin{array}{c}\text { Life } \\
\text { form }\end{array}$ & Geoelement & Status & Localities (finding sites) & $\begin{array}{c}\text { Previously recorded } \\
\text { findings }\end{array}$ \\
\hline Geranium macrorrhizum L. & Geraniaceae & $\mathrm{H}$ & ILBA-bap & & 32 & \\
\hline Geranium molle L. ssp. molle & Geraniaceae & $\mathrm{T}$ & WISP & & 27,47 & \\
\hline $\begin{array}{l}\text { Geranium molle L. ssp. brutium } \\
\text { (Gasparr.) Graebn. }\end{array}$ & Geraniaceae & $\mathrm{T}$ & ME-east & & $2,14,16,20,37,44,45,61,66,69,70$ & V\&al-2019 \\
\hline Geranium purpureum Vill. & Geraniaceae & $\mathrm{T}$ & SEU-me & & $\begin{array}{c}2,6,10,14,21,25,30,31,36,37,38 \\
40,41,42,45,50,51,52,53,55,56,70\end{array}$ & $\begin{array}{l}\text { M\&K-2016; VRZ-2012; } \\
\text { V\&al-2019 }\end{array}$ \\
\hline Geranium pusillum Burm. f. & Geraniaceae & $\mathrm{T}$ & EURO & & 20,27 & Ma-1964 \\
\hline Geranium robertianum $\mathrm{L}$. & Geraniaceae & $\mathrm{T}$ & WISP & & $\begin{array}{c}11,14,16,20,32,34,35,44,45,60,63 \\
64,69\end{array}$ & V\&al-2019 \\
\hline Geranium rotundifolium $\mathrm{L}$. & Geraniaceae & $\mathrm{T}$ & EUAS & & $2,14,27,37,49,68$ & Ma-1992; M\&K-2016 \\
\hline Geranium sanguineum $\mathrm{L}$. & Geraniaceae & $\mathrm{H}$ & SEU-me & & $10,11,19,20,36,39,65,66,67$ & Pe-1832:62; V\&al-2019 \\
\hline Geum urbanum L. & Rosaceae & $\mathrm{H}$ & WISP & & $\begin{array}{c}3,11,14,20,27,32,34,35,36,37,38 \\
40,42,47,52,58,60,61,63,64,65,66 \\
70\end{array}$ & Ma-1992 \\
\hline Gladiolus imbricatus L. & Iridaceae & G & CUAD & $\mathrm{sp}$ & 29 & \\
\hline Gladiolus illyricus W. D. J. Koch & Iridaceae & G & SEU-me & $\mathrm{sp}$ & 28 & V\&al-2019 \\
\hline Glechoma hederacea L. & Lamiaceae & $\mathrm{H}$ & $\mathrm{CIHO}$ & & 14,16 & Vi-1847:203 \\
\hline $\begin{array}{l}\text { Globularia cordifolia L. ssp. } \\
\text { bellidifolia (Ten.) Wettst. }\end{array}$ & Globulariaceae & $\mathrm{Ch}$ & ME-ilape & & $\begin{array}{c}2,7,8,9,10,11,12,26,29,30,31,33 \\
36,39,40,41,47,48,50,51,52,53,54 \\
\quad 55,56,57,58,59,64,67,69,70\end{array}$ & $\begin{array}{l}\text { Vi-1847:10 as G. } \\
\text { cordifolia; L\&R-1989, } \\
\text { LRS-1989 as G. } \\
\text { meridionalis (Podp.) } \\
\text { Schw.; Se\&al-2016; } \\
\text { V\&al-2019 }\end{array}$ \\
\hline $\begin{array}{l}\text { Globularia cordifolia L. ssp. } \\
\text { cordifolia (L.) Hayek }\end{array}$ & Globulariaceae & $\mathrm{Ch}$ & SEU-mo & & Significant Landscape of Sutina & V\&al-2019 \\
\hline Glyceria fluitans (L.) R. Br. & Poaceae & Hy & WISP & $\begin{array}{l}\text { VU, } \\
\text { sp }\end{array}$ & 70 & \\
\hline Glycyrrhiza glabra L. & Fabaceae & G & ME-po & & 35 & \\
\hline Gratiola officinalis L. & Scrophulariaceae & $\mathrm{H}$ & WISP & & 28 & \\
\hline Gymnadenia conopsea (L.) R. Br. & Orchidaceae & G & EUAS & $\mathrm{sp}$ & 41,46 & Kr-2005:120 \\
\hline Gypsophila fastigiata L. & Caryophyllaceae & $\mathrm{Ch}$ & CEU & $\begin{array}{c}\mathrm{DD}, \\
\mathrm{sp}\end{array}$ & 51,52 & \\
\hline $\begin{array}{l}\text { Haplophyllum patavinum (L.) } \\
\text { G.Don }\end{array}$ & Rutaceae & $\mathrm{Ch}$ & ME-ilseu & & $\begin{array}{c}1,9,26,31,34,36,39,41,56,58,62 \\
65,70\end{array}$ & V\&al-2019 \\
\hline Hedera helix $\mathrm{L}$. & Araliaceae & $\mathrm{P}$ & EUAS & & $\begin{array}{c}1,3,4,5,13,14,16,20,23,25,27,28 \\
29,37,42,43,45,46,47,50,61,63,64 \\
69,70\end{array}$ & $\begin{array}{l}\text { Je-1952; L\&al-1987; } \\
\text { V\&al-2019 }\end{array}$ \\
\hline Helianthemum canum (L.) Baumg. & Cistaceae & $\mathrm{Ch}$ & EURO & & around the Golubinka pit & VRZ-2012 \\
\hline $\begin{array}{l}\text { Helianthemum nummularium (L.) } \\
\text { Mill. ssp. glabrum (Koch) Wilczek }\end{array}$ & Cistaceae & $\mathrm{Ch}$ & SEU-me & & 26 & \\
\hline $\begin{array}{l}\text { Helianthemum nummularium (L.) } \\
\text { Mill. ssp. grandiflorum (Scop.) } \\
\text { Schinz et Thell. }\end{array}$ & Cistaceae & $\mathrm{Ch}$ & SEU-mo & & $1,9,10,11,17,42,45,47,49$ & V\&al-2019 \\
\hline $\begin{array}{l}\text { Helianthemum nummularium (L.) } \\
\text { Mill. ssp. obscurum (Čelak.) } \\
\text { Holub }\end{array}$ & Cistaceae & $\mathrm{Ch}$ & SEU-me & & $16,18,19,26,32,38,46,67,70$ & \\
\hline $\begin{array}{l}\text { Helianthemum oelandicum (L.) DC. } \\
\text { ssp. italicum (L.) Font Quer et } \\
\text { Rothm. }\end{array}$ & Cistaceae & $\mathrm{Ch}$ & SEU-me & & $\begin{array}{c}2,11,13,31,36,39,41,48,51,52,54 \\
58,64,69\end{array}$ & V\&al-2019 \\
\hline $\begin{array}{l}\text { Helianthemum salicifolium (L.) } \\
\text { Mill. }\end{array}$ & Cistaceae & $\mathrm{T}$ & SEU-me & & $1,2,5,29,44$ & \\
\hline Helianthus annuus L. & Asteraceae & $\mathrm{T}$ & CUAD & & $24,43^{*}$ & \\
\hline Helianthus tuberosus L. & Asteraceae & G & CUAD & inv & $3,4,5,13,14,27,28,46,70$ & Vi-1847:94 \\
\hline $\begin{array}{l}\text { Helichrysum italicum (Roth) G. } \\
\text { Don }\end{array}$ & Asteraceae & $\mathrm{Ch}$ & ME-circ & & $\begin{array}{c}1,2,6,8,21,25,26,27,43,44,45,47 \\
48,50,54,55,57,61,62,64\end{array}$ & Se\&al-2016; V\&al-2019 \\
\hline $\begin{array}{l}\text { Helictotrichon convolutum } \\
\text { (C.Presl) Henrard. }\end{array}$ & Poaceae & $\mathrm{H}$ & ME-west & & $1,2,6,23,29,55,58$ & \\
\hline Heliotropium europaeum L. & Boraginaceae & $\mathrm{T}$ & ME-po & & $3,4,13,14,27,49,68$ & \\
\hline $\begin{array}{l}\text { Helleborus multifidus Vis. ssp. } \\
\text { multifidus }\end{array}$ & Ranunculaceae & G & ME-ilape & $\begin{array}{l}\text { end, } \\
\text { sp }\end{array}$ & $\begin{array}{c}7,8,9,10,11,12,13,14,15,16,17,18, \\
19,20,34,35,36,37,38,39,40,41,44, \\
47,51,52,53,55,56,57,58,59,61,62, \\
64,65,66,67\end{array}$ & $\begin{array}{l}\text { Pe-1832:65, Je-1952 and } \\
\text { V\&al-2019 as H. } \\
\text { multifidus }\end{array}$ \\
\hline Hemerocallis fulva (L.) L. & Xanthorrhoeaceae & G & CUAD & & $1,5,24,28,42,46,66^{*}, 70$ & \\
\hline
\end{tabular}




\begin{tabular}{|c|c|c|c|c|c|c|}
\hline Taxon & Family & \begin{tabular}{|c|} 
Life \\
form
\end{tabular} & Geoelement & Status & Localities (finding sites) & $\begin{array}{l}\text { Previously recorded } \\
\text { findings }\end{array}$ \\
\hline Heracleum sphondylium L. & Apiaceae & $\mathrm{H}$ & EUAS & & $34,65,66$ & Vi-1852:54 \\
\hline Herniaria glabra $\mathrm{L}$. & Caryophyllaceae & $\mathrm{T}$ & EUAS & & $11,39,40,55$ & L\&R-1989 \\
\hline Herniaria hirsuta L. ssp. hirsuta & Caryophyllaceae & $\mathrm{T}$ & EUAS & & 3 & \\
\hline Herniaria incana Lam. & Caryophyllaceae & $\mathrm{H}$ & SEU-me & & $1,22,25,26,27,30$ & V\&al-2019 \\
\hline Hesperis laciniata All. & Brassicaceae & $\mathrm{H}$ & ME-ilseu & & 14,41 & \\
\hline Hibiscus trionum $\mathrm{L}$. & Malvaceae & $\mathrm{T}$ & SEU-po & $\mathrm{EN}, \mathrm{sp}$ & $1,13,68$ & \\
\hline Hieracium cymosum L. & Cichoriaceae & $\mathrm{H}$ & EUAS & & 2 & \\
\hline $\begin{array}{l}\text { Hieracium heterogynum (Froel.) } \\
\text { Gutermann }\end{array}$ & Cichoriaceae & $\mathrm{H}$ & ILBA-end & & $6,26,27,31,39,43,51,53,54,56,58$ & V\&al-2019 \\
\hline $\begin{array}{l}\text { Hieracium hoppeanum Schult. ssp. } \\
\text { troicum Zahn }\end{array}$ & Cichoriaceae & $\mathrm{H}$ & SEEU & & $\begin{array}{c}1,8,17,18,19,21,23,24,25,26,31 \\
33,34,35,39,41,43,48,49,50,53,54 \\
55,56,57,59,62,66\end{array}$ & \\
\hline Hieracium murorum L. & Cichoriaceae & $\mathrm{H}$ & EUAS & & 38,64 & Vi-1847:123; V\&al-2019 \\
\hline Hieracium pilosella $\mathrm{L}$. & Cichoriaceae & $\mathrm{H}$ & EUAS & & $11,51,58$ & V\&al-2019 \\
\hline $\begin{array}{l}\text { Hieracium piloselloides Vill. ssp. } \\
\text { piloselloides }\end{array}$ & Cichoriaceae & $\mathrm{H}$ & ME-ilseu & & $8,31,32,51,59,69$ & \\
\hline $\begin{array}{l}\text { Hieracium praealtum Vill. ex } \\
\text { Gochnat ssp. bauhinii (Besser) } \\
\text { Petunn. }\end{array}$ & Cichoriaceae & $\mathrm{H}$ & EUAS & & \begin{tabular}{|c|}
$11,13,17,21,25,29,31,35,39,40,42$ \\
$43,45,46,47,53,55,57,58,62,63,64$ \\
70
\end{tabular} & $\begin{array}{l}\text { Tr-1992 as H. bauhinii } \\
\text { Schultes; V\&al-2019 }\end{array}$ \\
\hline $\begin{array}{l}\text { Hieracium racemosum Waldst. et } \\
\text { Kit. ex Willd. }\end{array}$ & Cichoriaceae & $\mathrm{H}$ & SEU-me & & 19,46 & \\
\hline Hieracium tommasinii Rchb. f. & Cichoriaceae & $\mathrm{H}$ & ME-ilade & & $\begin{array}{l}\text { rocks of the eastern slopes of Svilaja; } \\
\text { SL Sutina }\end{array}$ & $\begin{array}{l}\text { L\&al-1987 as H. } \\
\text { tomasinii Kern. s.l.; } \\
\text { V\&al-2019 }\end{array}$ \\
\hline Hieracium waldsteinii Tausch & Cichoriaceae & $\mathrm{H}$ & ILBA-end & $\begin{array}{l}\text { end, } \\
\text { sp }\end{array}$ & $32,33,34,35,39,40,41,53,55$ & \\
\hline Hippocrepis comosa $\mathrm{L}$. & Fabaceae & $\mathrm{H}$ & SEU-me & & $\begin{array}{c}2,8,17,29,30,31,33,37,42,44,45 \\
46,47,48,51,53,58,60,61,62,64,65 \\
69,70\end{array}$ & V\&al-2019 \\
\hline Holcus lanatus L. & Poaceae & $\mathrm{H}$ & EUAS & & 16,28 & Vi-1842:48 \\
\hline Hordeum bulbosum L. & Poaceae & $\mathrm{H}$ & SEU-me & & $17,25,28$ & \\
\hline $\begin{array}{l}\text { Hordeum murinum L. ssp. } \\
\text { leporinum (Link) Arcang. }\end{array}$ & Poaceae & $\mathrm{T}$ & ME-circ & & $16,27,37,45$ & $\begin{array}{l}\text { Ma-1964,1992 as H. } \\
\text { leporinum Lk.; } \\
\text { V\&al-2019 }\end{array}$ \\
\hline $\begin{array}{l}\text { Hordeum murinum L. ssp. } \\
\text { murinum }\end{array}$ & Poaceae & $\mathrm{T}$ & WISP & LC & $3,5,14,16,20,23$ & \\
\hline Hordeum secalinum Schreb. & Poaceae & $\mathrm{H}$ & EUAS & $\mathrm{EN}, \mathrm{sp}$ & 43 & \\
\hline Hornungia petraea (L.) Rchb. & Brassicaceae & $\mathrm{T}$ & WISP & & $8,9,10,11,12,38,51,52$ & V\&al-2019 \\
\hline Humulus lupulus L. & Cannabaceae & $\mathrm{H}$ & $\mathrm{CIHO}$ & & $14,15,37,70$ & \\
\hline $\begin{array}{l}\text { Hyacinthella dalmatica (Baker) } \\
\text { Chouard }\end{array}$ & Asparagaceae & G & ME-ilade & $\begin{array}{l}\text { end, } \\
\text { DD, } \\
\text { sp }\end{array}$ & $2,5,6,13,30,41,44,51,52,53,66,67$ & M\&al-2013; V\&al-2019 \\
\hline $\begin{array}{l}\text { Himantoglossum adriaticum } \mathrm{H} \text {. } \\
\text { Baumann }\end{array}$ & Orchidaceae & G & SEU-me & NT, sp & 1 & \\
\hline $\begin{array}{l}\text { Hymenolobus procumbens (L.) } \\
\text { Nutt. }\end{array}$ & Fabaceae & $\mathrm{T}$ & WISP & DD & 41 & \\
\hline Hyoscyamus albus L. & Solanaceae & $\mathrm{H}$ & ME-circ & & $21,23,30,59$ & \\
\hline Hyoscyamus niger L. & Solanaceae & $\mathrm{H}$ & EUAS & & 59 & Pe-1832:68 \\
\hline Hypericum montanum L. & Clusiaceae & $\mathrm{H}$ & EUAS & & 32,35 & V\&al-2019 \\
\hline $\begin{array}{l}\text { Hypericum perforatum L. ssp. } \\
\text { perforatum }\end{array}$ & Clusiaceae & $\mathrm{H}$ & SEU-me & & $\begin{array}{c}1,2,3,4,5,7,8,9,10,11,13,15,17 \\
19,20,21,22,23,25,26,27,28,29,32, \\
33,34,35,36,37,38,39,40,41,42,43 \\
44,45,47,48,49,50,51,52,53,54,55 \\
58,59,60,63,65,66,69\end{array}$ & $\begin{array}{l}\text { V\&al-2019 as } H . \\
\text { perforatum L. }\end{array}$ \\
\hline Hyssopus officinalis L. & Lamiaceae & $\mathrm{Ch}$ & SEU-me & DD & 6 & \\
\hline Ilex aquifolium $\mathrm{L}$. & Aquifoliaceae & $\mathrm{P}$ & SEU-atl & $\begin{array}{l}\mathrm{VU}, \\
\mathrm{sp}\end{array}$ & 63 & V\&al-2019 \\
\hline Inula britannica $\mathrm{L}$. & Asteraceae & $\mathrm{H}$ & EUAS & & $13,22,50$ & \\
\hline Inula conyza DC. & Asteraceae & $\mathrm{H}$ & SEU-po & & $\begin{array}{c}3,5,13,21,23,27,34,35,36,39,40 \\
43,47,49,50,53,54,58,59,61,65,66\end{array}$ & V\&al-2019 \\
\hline Inula ensifolia L. & Asteraceae & $\mathrm{H}$ & EEUPO & & $\begin{array}{c}9,11,12,26,31,39,40,41,50,51,52 \\
53,56,65\end{array}$ & Se\&al-2016; V\&al-2019 \\
\hline
\end{tabular}




\begin{tabular}{|c|c|c|c|c|c|c|}
\hline Taxon & Family & \begin{tabular}{|c|} 
Life \\
form
\end{tabular} & Geoelement & Status & Localities (finding sites) & $\begin{array}{l}\text { Previously recorded } \\
\text { findings }\end{array}$ \\
\hline Inula hirta $\mathrm{L}$. & Asteraceae & $\mathrm{H}$ & SEU-me & & $51,58,65$ & V\&al-2019 \\
\hline Inula montana L. & Asteraceae & $\mathrm{H}$ & SEU-atl & & "circa Verlika" & Vi-1847:64 \\
\hline Inula oculus-christ $\mathrm{L}$ & Asteraceae & $\mathrm{H}$ & SEU-po & & $5,15,16,17,18,21,27,43,46,50,58$ & V\&al-2019 \\
\hline Inula salicina L. ssp. salicina & Asteraceae & $\mathrm{H}$ & EUAS & LC & $23,25,28$ & \\
\hline Inula spiraeifolia $\mathrm{L}$. & Asteraceae & $\mathrm{H}$ & SEU-me & & $1,2,4,18,25,26,45$ & \\
\hline $\begin{array}{l}\text { Inula verbascifolia (Willd.) } \\
\text { Hausskn. ssp. verbascifolia }\end{array}$ & Asteraceae & $\mathrm{Ch}$ & ME-ilseu & & $6,14,41,48,50,54,57,58$ & $\begin{array}{l}\text { L\&al-1987 as I. } \\
\text { verbascifolia (Willd.) } \\
\text { Hausskn.; M\&K-2016; } \\
\text { V\&al-2019 }\end{array}$ \\
\hline Ipomoea purpurea Roth & Convolvulaceae & $\mathrm{T}$ & CUAD & & 1,27 & \\
\hline Iris adriatica Trinajstic ex Mitic & Iridaceae & G & ME-ilade & $\begin{array}{c}\text { end, } \\
\text { NT, sp }\end{array}$ & 6 & \\
\hline Iris germanica L. & Iridaceae & G & CUAD & $\mathrm{sp}$ & $\begin{array}{c}1,2,3^{*}, 16,17,20,22^{*}, 27,29,30,37 \\
46,66\end{array}$ & \\
\hline Iris illyrica Tomm. & Iridaceae & G & ME-ilade & $\begin{array}{l}\text { end, } \\
\text { LC, sp }\end{array}$ & 23,41 & V\&al-2019 \\
\hline Iris pseudacorus L. & Iridaceae & G & WISP & $\mathrm{sp}$ & 70 & \\
\hline Isatis tinctoria $\mathrm{L}$. & Brassicaceae & $\mathrm{H}$ & EUAS & & 5,23 & \\
\hline Isopyrum thalictroides L. & Ranunculaceae & G & EUAS & & Significant Landscape of Sutina & V\&al-2019 \\
\hline Juglans regia $\mathrm{L}$. & Juglandaceae & $P$ & CUAD & & $\begin{array}{c}1,3^{*}, 4,11^{*}, 13^{*}, 14,16,20,22,25,27 \\
30,37,42,43,46^{*}, 47,48,49^{*}, 50^{*}, 58 \\
59,61^{*}, 66,70\end{array}$ & \\
\hline Juncus articulatus L. & Juncaceae & $\mathrm{H}$ & $\mathrm{CIHO}$ & & 22 & \\
\hline Juncus bufonius L. & Juncaceae & $\mathrm{T}$ & WISP & & 28 & L\&R-1989 \\
\hline Juncus gerardi Loisel. ssp. gerardi & Juncaceae & G & WISP & & 46 & \\
\hline Juncus inflexus L. & Juncaceae & $\mathrm{H}$ & EUAS & & 23 & V\&al-2019 \\
\hline Juncus tenuis Willd. & Juncaceae & $\mathrm{H}$ & CUAD & inv & 59 & \\
\hline $\begin{array}{l}\text { Juniperus communis L. ssp. } \\
\text { communis }\end{array}$ & Cupressaceae & $P$ & $\mathrm{CIHO}$ & & 51,52 & $\begin{array}{l}\text { V\&al-2019 as J. } \\
\text { communis L. }\end{array}$ \\
\hline $\begin{array}{l}\text { Juniperus communis L. ssp. nana } \\
\text { Syme }\end{array}$ & Cupressaceae & $\mathrm{P}$ & EURO & & 8 & \\
\hline $\begin{array}{l}\text { Juniperus oxycedrus L. ssp. } \\
\text { macrocarpa (Sm.) Ball }\end{array}$ & Cupressaceae & $\mathrm{P}$ & ME-circ & LC & 31 & VRZ-2012; V\&al-2019 \\
\hline $\begin{array}{l}\text { Juniperus oxycedrus L. ssp. } \\
\text { oxycedrus }\end{array}$ & Cupressaceae & $\mathrm{P}$ & ME-circ & & $\begin{array}{r}1,2,3,7,17,21,23,30,31,33,34,37 \\
42,43,44,45,47,48,50,51,53,54,55 \\
56,57,58,59,60,61,62,64,67,69,70\end{array}$ & $\begin{array}{l}\text { Ja-1908, Je-1952, } \\
\text { Me-1972, L\&al-1987 and } \\
\text { Tr-1992, all as J. } \\
\text { oxycedrus L.; V\&al-2019 }\end{array}$ \\
\hline Jurinea mollis (L.) Rchb & Asteraceae & $\mathrm{H}$ & SEEU & & $39,40,41,51,52,53,54,56,58,64,67$ & Se\&al-2016; V\&al-2019 \\
\hline Kickxia spuria (L.) Dumort. & Scrophulariaceae & $\mathrm{T}$ & EUAS & & 13,22 & \\
\hline Knautia adriatica Ehrend & Dipsacaceae & $\mathrm{H}$ & ME-ilade & $\begin{array}{l}\text { end, } \\
\text { DD, } \\
\text { sp }\end{array}$ & 31,59 & \\
\hline Knautia arvensis (L.) Coult. & Dipsacaceae & $\mathrm{H}$ & EUAS & & $32,36,55,65$ & V\&al-2019 \\
\hline Knautia clementii (Beck) Ehrend. & Dipsacaceae & $\mathrm{H}$ & ME-ilade & $\begin{array}{l}\text { end, } \\
\text { DD, } \\
\text { sp }\end{array}$ & $26,36,46$ & \\
\hline Knautia dalmatica Beck & Dipsacaceae & $\mathrm{H}$ & ME-ilade & $\begin{array}{l}\text { end, } \\
\text { DD, } \\
\text { sp }\end{array}$ & 24,36 & L\&al-1987; LRS-1989 \\
\hline Knautia dinarica (Murb.) Borbás & Dipsacaceae & $\mathrm{H}$ & SEEU & & 38,46 & \\
\hline Knautia illyrica Beck & Dipsacaceae & $\mathrm{H}$ & ME-ilape & $\begin{array}{l}\text { end, } \\
\text { DD, } \\
\text { sp }\end{array}$ & 26,53 & L\&al-1987; Tr-1992, \\
\hline Knautia travnicensis (Beck) Szabó & Dipsacaceae & $\mathrm{T}$ & ME-circ & $\begin{array}{l}\text { end, } \\
\text { DD, } \\
\text { sp }\end{array}$ & $3,21,46$ & \\
\hline $\begin{array}{l}\text { Koeleria macrantha (Ledeb.) } \\
\text { Schult. }\end{array}$ & Poaceae & $\mathrm{H}$ & $\mathrm{CIHO}$ & & Significant Landscape of Sutina & V\&al-2019 \\
\hline $\begin{array}{l}\text { Koeleria pyramidata (Lam.) P. } \\
\text { Beauv. }\end{array}$ & Poaceae & $\mathrm{H}$ & CEU & & $9,11,23,31,33,40$ & \\
\hline Koeleria splendens C.Presl & Poaceae & $\mathrm{H}$ & SEU-me & & $\begin{array}{c}1,6,7,8,17,18,25,26,31,32,38,39 \\
45,48,50,51,52,55,56,59,60,66,69\end{array}$ & $\begin{array}{l}\text { LRS-1989; Tr-1992; } \\
\text { Se\&al-2016; V\&al-2019 }\end{array}$ \\
\hline
\end{tabular}




\begin{tabular}{|c|c|c|c|c|c|c|}
\hline Taxon & Family & \begin{tabular}{|c|} 
Life \\
form
\end{tabular} & Geoelement & Status & Localities (finding sites) & $\begin{array}{l}\text { Previously recorded } \\
\text { findings }\end{array}$ \\
\hline Lactuca perennis L. & Cichoriaceae & $\mathrm{H}$ & SEU-po & & 35,36 & Ja-1908 \\
\hline Lactuca sativa L. & Cichoriaceae & $\mathrm{T}$ & CUAD & & $\begin{array}{c}11,16^{*}, 22^{*}, 25^{*}, 25,27,37^{*}, 37,43^{*} \\
49^{*}, 70\end{array}$ & \\
\hline Lactuca serriola $\mathrm{L}$. & Cichoriaceae & $\mathrm{H}$ & WISP & & $\begin{array}{c}1,3,4,5,11,13,14,15,16,17,20,22 \\
23,25,27,28,29,37,38,43,45,48,50 \\
54,59,69,70\end{array}$ & V\&al-2019 \\
\hline Lactuca viminea (L.) J. et C.Presl & Cichoriaceae & $\mathrm{H}$ & SEU-po & & $\begin{array}{r}1,2,3,4,5,6,8,13,14,15,16,20,21, \\
22,23,25,26,27,28,29,31,32,35,37, \\
38,40,42,43,45,50,53,55,56,58,66\end{array}$ & V\&al-2019 \\
\hline Lamium amplexicaule $\mathrm{L}$. & Lamiaceae & $\mathrm{T}$ & EUAS & & $14,16,24,27,28,29,37,40,65$ & M\&K-2016 \\
\hline Lamium bifidum Cirillo & Lamiaceae & $\mathrm{T}$ & ME-circ & & 2 & Vi-1847:211; V\&al-2019 \\
\hline Lamium galeobdolon (L.) L. & Lamiaceae & $\mathrm{H}$ & CEU & & 14,32 & $\begin{array}{l}\text { Vi-1847:211 as } L . \\
\text { galeobdolon Crantz }\end{array}$ \\
\hline Lamium maculatum L. & Lamiaceae & $\mathrm{H}$ & EUAS & & $\begin{array}{c}10,13,14,16,27,30,34,37,42,44,45 \\
47,50,66,70\end{array}$ & V\&al-2019 \\
\hline Lamium purpureum $\mathrm{L}$. & Lamiaceae & $\mathrm{T}$ & EUAS & & $\begin{array}{c}2,20,26,29,36,39,40,42,44,60,61 \\
69,70\end{array}$ & \\
\hline $\begin{array}{l}\text { Lappula squarrosa (Retz.) Gurke } \\
\text { ssp. squarrosa }\end{array}$ & Boraginaceae & $\mathrm{T}$ & EUAS & & 26 & V\&al-2019 \\
\hline Lapsana communis L. & Cichoriaceae & $\mathrm{T}$ & EUAS & & 32,35 & V\&al-2019 \\
\hline Laser trilobum (L.) Borkh. & Apiaceae & $\mathrm{H}$ & SEU-po & & 32 & \\
\hline Laserpitium latifolium L. & Apiaceae & $\mathrm{H}$ & EURO & & 24 & \\
\hline Lathraea squamaria L. & Scrophulariaceae & G & EUAS & & 14 & \\
\hline Lathyrus annuus L. & Fabaceae & $\mathrm{T}$ & SEU-me & & 14 & Vi-1852:327 \\
\hline Lathyrus aphaca L. & Fabaceae & $\mathrm{T}$ & SEU-me & & $5,14,16,17,21,27,28,37,42,44,69$ & V\&al-2019 \\
\hline Lathyrus cicera $\mathrm{L}$. & Fabaceae & $\mathrm{T}$ & ME-circ & & 42,45 & \\
\hline Lathyrus latifolius L. & Fabaceae & $\mathrm{H}$ & SEU-me & & $\begin{array}{c}2,4,13,15,20,21,22,28,37,42,46 \\
58,61,65,69\end{array}$ & V\&al-2019 \\
\hline Lathyrus niger (L.) Bernh. & Fabaceae & G & EURO & & Significant Landscape of Sutina & V\&al-2019 \\
\hline $\begin{array}{l}\text { Lathyrus pannonicus (Jacq.) } \\
\text { Garcke }\end{array}$ & Fabaceae & G & EEUPO & & $2,29,30$ & V\&al-2019 \\
\hline Lathyrus pratensis L. & Fabaceae & $\mathrm{H}$ & EUAS & & $11,15,65,70$ & V\&al-2019 \\
\hline Lathyrus sphaericus Retz. & Fabaceae & $\mathrm{T}$ & ME-circ & & $21,25,42,47,70$ & V\&al-2019 \\
\hline Lathyrus sylvestris L. & Fabaceae & $\mathrm{H}$ & EURO & & 28 & \\
\hline Lathyrus tuberosus L. & Fabaceae & G & EUAS & & 70 & Vi-1852:329 \\
\hline Lathyrus venetus (Mill.) Wohlf. & Fabaceae & G & EEUPO & & 32 & V\&al-2019 \\
\hline Lathyrus vernus (L.) Bernhardt & Fabaceae & G & EEUPO & & $11,14,36,52,67$ & \\
\hline Legousia hybrida (L.) Delarbre & Campanulaceae & $\mathrm{T}$ & SEU-atl & & $25,29,46$ & \\
\hline $\begin{array}{l}\text { Legousia speculum-veneris (L.) } \\
\text { Chaix }\end{array}$ & Campanulaceae & $\mathrm{T}$ & SEU-me & & 67 & \\
\hline Leontodon crispus Vill. ssp. crispus & Cichoriaceae & $\mathrm{H}$ & SEU-me & & $\begin{array}{c}8,9,11,17,23,30,31,33,36,39,45 \\
52,59,61,62\end{array}$ & $\begin{array}{l}\text { Tr-1992, V\&al-2019, as } \\
\text { L. crispus Vill. }\end{array}$ \\
\hline $\begin{array}{l}\text { Leontodon crispus Vill. ssp. } \\
\text { rossianus (Degenet Lengyel) } \\
\text { Hayek }\end{array}$ & Cichoriaceae & $\mathrm{H}$ & ME-ilade & $\begin{array}{c}\text { end, } \\
\text { sp }\end{array}$ & 2 & \\
\hline $\begin{array}{l}\text { Leontodon hispidus L. ssp. } \\
\text { danubialis (Jacq.) Simonk. }\end{array}$ & Cichoriaceae & $\mathrm{H}$ & CEU & & $15,64,70$ & $\begin{array}{l}\text { V\&al-2019 as L. hispidus } \\
\text { L. }\end{array}$ \\
\hline Leonurus cardiaca L. & Lamiaceae & $\mathrm{H}$ & EUAS & & $3,5,16,21,23,27,50$ & \\
\hline Lepidium campestre (L.) R. Br. & Brassicaceae & $\mathrm{T}$ & WISP & & $3,16,17,43,45,46,63,70$ & \\
\hline Lepidium graminifolium $\mathrm{L}$. & Brassicaceae & $\mathrm{H}$ & SEU-po & & 14,27 & \\
\hline Lepidium virginicum $\mathrm{L}$. & Brassicaceae & $\mathrm{T}$ & CUAD & inv & Vrlika & Ma-1964 \\
\hline $\begin{array}{l}\text { Leucanthemum illyricum } \\
\text { (Horvatić) Vogt et Greuter }\end{array}$ & Asteraceae & $\mathrm{H}$ & ME-ilade & & Svilaja mountain range & LRS-1989 \\
\hline Leucanthemum ircutianum DC. & Asteraceae & $\mathrm{H}$ & SEU-me & & $\begin{array}{l}2,14,16,23,29,34,37,39,41,42,44 \\
45,47,53,55,60,61,63,64,66,69,70\end{array}$ & \\
\hline Leucanthemum vulgare Lam. & Asteraceae & $\mathrm{H}$ & EUAS & & $33,40,53$ & $\begin{array}{l}\text { Pe-1832:36 as } \\
\text { Chrysanthemum } \\
\text { leucanthemum L.; } \\
\text { V\&al-2019 }\end{array}$ \\
\hline Ligustrum vulgare $\mathrm{L}$. & Oleaceae & $\mathrm{P}$ & CEU & & $4,16,22,37,43,46,47,61,64,69,70$ & $\begin{array}{l}\text { Vi-1852:20; L\&al-1987; } \\
\text { V\&al-2019 }\end{array}$ \\
\hline
\end{tabular}




\begin{tabular}{|c|c|c|c|c|c|c|}
\hline Taxon & Family & \begin{tabular}{|c|} 
Life \\
form
\end{tabular} & Geoelement & Status & Localities (finding sites) & $\begin{array}{l}\text { Previously recorded } \\
\text { findings }\end{array}$ \\
\hline Lilium bulbiferum $\mathrm{L}$. & Liliaceae & G & $\mathrm{CEU}$ & $\begin{array}{l}\mathrm{VU}, \\
\mathrm{sp}\end{array}$ & Svilaja mountain range & $\begin{array}{l}\text { LRS- } 1989 \text { as } L . \\
\text { bulbiferum } \text { L. ssp. } \\
\text { croceum (Ch.) Bak. }\end{array}$ \\
\hline Lilium candidum $\mathrm{L}$. & Liliaceae & G & CUAD & & $3,23,24^{*}, 27^{*}, 37^{*}, 43^{*}$ & \\
\hline Lilium carniolicum Bernh. ex Koch & Liliaceae & G & ILBA-end & $\begin{array}{l}\text { VU, } \\
\text { sp }\end{array}$ & 41 & V\&al-2019 \\
\hline $\begin{array}{l}\text { Lilium martagon L. ssp. cattaniae } \\
\text { (Vis.) Degen }\end{array}$ & Liliaceae & G & ME-ilade & $\begin{array}{l}\text { end, } \\
\text { VU, } \\
\text { sp }\end{array}$ & $\begin{array}{c}11,32,35,36,38,39,40,41,52,53,65 \\
67\end{array}$ & \begin{tabular}{|l|} 
Vi-1842:132 as $L$. \\
martagon; Ku-1969 as L. \\
cattaniae Vis.; V\&al-2019 \\
\end{tabular} \\
\hline Limodorum abortivum (L.) Sw & Orchidaceae & G & SEU-me & $\mathrm{sp}$ & $13,14,18,25,41,70$ & $\begin{array}{l}\text { Kr-2005:130; Df-2006; } \\
\text { V\&al-2019 }\end{array}$ \\
\hline $\begin{array}{l}\text { Linaria angustissima (Loisel.) } \\
\text { Borbás }\end{array}$ & Scrophulariaceae & $\mathrm{H}$ & SEU-me & & $5,7,15,27,59$ & $\begin{array}{l}\text { Vi-1847:163 as L. italica } \\
\text { Trevir. }\end{array}$ \\
\hline Linaria vulgaris Mill. & Scrophulariaceae & $\mathrm{H}$ & EUAS & & 50,68 & V\&al-2019 \\
\hline Linum austriacum $\mathrm{L}$. & Linaceae & $\mathrm{H}$ & EEUPO & & $29,31,37,42,44,45,57,64,67,69,70$ & V\&al-2019 \\
\hline Linum bienne Mill. & Linaceae & $\mathrm{H}$ & ME-atl & & $28,43,45,52,56,58,60$ & \\
\hline Linum catharticum $\mathrm{L}$. & Linaceae & $\mathrm{T}$ & WISP & & $7,8,32,34,35,40,46$ & \\
\hline Linum hirsutum L. & Linaceae & $\mathrm{H}$ & SEEU & & 69 & \\
\hline Linum hologynum Rchb. & Linaceae & $\mathrm{H}$ & ILBA-end & & 16 & \\
\hline Linum perenne $\mathrm{L}$. & Linaceae & $\mathrm{H}$ & SEU-mo & & Svilaja mountain range & $\begin{array}{l}\text { LRS-1989 as L. } \\
\text { montanum Schl. ex DC. }\end{array}$ \\
\hline Linum tenuifolium $\mathrm{L}$. & Linaceae & $\mathrm{Ch}$ & SEU-po & & $\begin{array}{c}5,7,8,11,19,23,26,28,31,32,34,38 \\
39,40,41,46,48,50,51,53,55,56,57, \\
58,62,65\end{array}$ & Tr-1992; V\&al-2019 \\
\hline Listera ovata (L.) R. Br. & Orchidaceae & G & EUAS & $\mathrm{sp}$ & plateau of Svilaja, Zelovo; SL Sutina & Kr-2005:134; V\&al-2019 \\
\hline Lithospermum arvense $\mathrm{L}$. & Boraginaceae & $\mathrm{T}$ & EUAS & & 37,69 & V\&al-2019 \\
\hline Lithospermum officinale $\mathrm{L}$. & Boraginaceae & $\mathrm{H}$ & EUAS & & 42,63 & V\&al-2019 \\
\hline $\begin{array}{l}\text { Lithospermum purpurocaeruleum } \\
\text { L. }\end{array}$ & Boraginaceae & $\mathrm{H}$ & SEU-po & & $2,20,26,64,65,67,70$ & V\&al-2019 \\
\hline Lolium multiflorum Lam. & Poaceae & $\mathrm{T}$ & ME-circ & & $5,24,58$ & \\
\hline Lolium perenne $\mathrm{L}$. & Poaceae & $\mathrm{H}$ & EURO & & $\begin{array}{c}1,3,5,13,15,16,20,22,23,25,27,28 \\
46,49,50,63,66\end{array}$ & V\&al-2019 \\
\hline $\begin{array}{l}\text { Lolium rigidum Gaudin ssp. } \\
\text { rigidum }\end{array}$ & Poaceae & $\mathrm{T}$ & ME-circ & & $14,16,22$ & $\begin{array}{l}\text { Ma-1992 as L. rigidum } \\
\text { Gaudin }\end{array}$ \\
\hline $\begin{array}{l}\text { Lomelosia argentea (L.) Greuter et } \\
\text { Burdet }\end{array}$ & Dipsacaceae & $\mathrm{H}$ & EEUPO & & Significant Landscape of Sutina & V\&al-2019 \\
\hline $\begin{array}{l}\text { Lomelosia graminifolia (L.) Greuter } \\
\text { et Burdet }\end{array}$ & Dipsacaceae & $\mathrm{H}$ & SEU-me & & $34,39,41,51,52$ & \\
\hline Lonicera caprifolium L. & Caprifoliaceae & $\mathrm{P}$ & SEU-co & & 52 & \\
\hline Lonicera etrusca Santi & Caprifoliaceae & $\mathrm{P}$ & ME-circ & & $\begin{array}{c}2,6,27,30 ?, 31,36,41,44,47 ?, 48,51 \\
55,57,58,67\end{array}$ & V\&al-2019 \\
\hline Lonicera xylosteum L. & Caprifoliaceae & $\mathrm{P}$ & EUAS & & 32 & Vi-1852:18 \\
\hline Lophochloa cristata (L.) Hyl. & Poaceae & $\mathrm{T}$ & ME-atl & & $3,23,25$ & \\
\hline Loranthus europaeus Jacq. & Loranthaceae & $\mathrm{P}$ & EUAS & & $13,30,45$ & Vi-1852:23 as V. album L. \\
\hline $\begin{array}{l}\text { Lotus corniculatus L. ssp. } \\
\text { corniculatus }\end{array}$ & Fabaceae & $\mathrm{H}$ & WISP & & $8,16,20,23,26,28,37,42,59,63,70$ & $\begin{array}{l}\text { Je-1952 and L\&R-1989 as } \\
\text { L. corniculatus L. }\end{array}$ \\
\hline $\begin{array}{l}\text { Lotus corniculatus L. ssp. hirsutus } \\
\text { Rothm. }\end{array}$ & Fabaceae & $\mathrm{H}$ & SEU-me & & $\begin{array}{c}1,5,7,8,9,11,16,17,21,23,25,26 \\
27,29,32,35,36,38,39,40,42,43,44 \\
45,46,52,53,55,58,62,65\end{array}$ & V\&al-2019 \\
\hline Lotus glaber Mill. & Fabaceae & $\mathrm{H}$ & WISP & & $13,15,21,22,28$ & \\
\hline Lunaria annua L. & Brassicaceae & $\mathrm{H}$ & ILBA-bap & & $23^{*}, 30$ & \\
\hline Luzula campestris (L.) DC. & Juncaceae & $\mathrm{H}$ & WISP & & $10,11,35,36,37,51,52,65$ & V\&al-2019 \\
\hline Luzula forsteri (Sm.) DC. & Juncaceae & $\mathrm{H}$ & ME-circ & & $25,37,63$ & \\
\hline $\begin{array}{l}\text { Luzula luzuloides (Lam.) Dandy et } \\
\text { Wilmott ssp. luzuloides }\end{array}$ & Juncaceae & $\mathrm{H}$ & CEU & & 32 & \\
\hline Luzula multiflora (Retz.) Lej. & Juncaceae & $\mathrm{H}$ & $\mathrm{CIHO}$ & & $2,25,44$ & \\
\hline Luzula sylvatica (Huds.) Gaudin & Juncaceae & $\mathrm{H}$ & SEEU & & Significant Landscape of Sutina & V\&al-2019 \\
\hline Lycopus europaeus L. & Lamiaceae & $\mathrm{H}$ & EUAS & & 70 & \\
\hline Lysimachia punctata L. & Primulaceae & $\mathrm{H}$ & EEUPO & & Significant Landscape of Sutina & V\&al-2019 \\
\hline Lythrum salicaria $\mathrm{L}$. & Lythraceae & $\mathrm{H}$ & WISP & & 4,22 & \\
\hline
\end{tabular}




\begin{tabular}{|c|c|c|c|c|c|c|}
\hline Taxon & Family & \begin{tabular}{|c|} 
Life \\
form
\end{tabular} & Geoelement & Status & Localities (finding sites) & $\begin{array}{l}\text { Previously recorded } \\
\text { findings }\end{array}$ \\
\hline $\begin{array}{l}\text { Malcolmia orsiniana (Ten.) Ten. } \\
\text { ssp. angustifolia (Boiss. et Orph.) } \\
\text { Stork }\end{array}$ & Brassicaceae & $\mathrm{H}$ & ILBA-end & & 10 & \\
\hline Malus pumila Mill. & Rosaceae & $\mathrm{P}$ & CUAD & & $\begin{array}{c}16,27^{*}, 29,37^{*}, 44,46,61,64,66^{*}, 69 \\
70\end{array}$ & $\begin{array}{l}\text { V\&al-2019 as } M \text {. } \\
\text { domestica Borkh. }\end{array}$ \\
\hline Malus sylvestris (L.) Mill. & Rosaceae & $\mathrm{P}$ & CEU & & Significant Landscape of Sutina & V\&al-2019 \\
\hline Malva neglecta Wallr. & Malvaceae & $\mathrm{T}$ & WISP & & 23,50 & \\
\hline Malva nicaeensis All. & Malvaceae & $\mathrm{H}$ & ME-circ & & 49 & \\
\hline Malva pusilla Sm. & Malvaceae & $\mathrm{T}$ & EUAS & & Vrlika & Ma-1964 \\
\hline Malva sylvestris L. & Malvaceae & $\mathrm{H}$ & WISP & & $\begin{array}{c}1,3,5,13,14,15,17,20,21,23,25,27 \\
28,37,43,50,65,66\end{array}$ & V\&al-2019 \\
\hline Marrubium incanum Desr. & Lamiaceae & $\mathrm{H}$ & ME-ilape & & $\begin{array}{c}1,2,3,5,7,13,15,16,17,18,20,21 \\
22,23,25,26,27,29,30,40,43,45,48 \\
49,51,54,58,59,61,66,70\end{array}$ & Tr-1992; V\&al-2019 \\
\hline Marrubium vulgare L. & Lamiaceae & $\mathrm{H}$ & WISP & & $1,5,6,8,13,21,27,49,50,54,66$ & \\
\hline Matthiola fruticulosa (L.) Maire & Brassicaceae & $\mathrm{Ch}$ & SEU-me & & 41,51 & Se\&al-2016 \\
\hline Medicago arabica (L.) Huds. & Fabaceae & $\mathrm{T}$ & WISP & & $14,16,69$ & \\
\hline Medicago falcata $\mathrm{L}$. & Fabaceae & $\mathrm{H}$ & EUAS & & $14,25,28,46,53,65,66$ & V\&al-2019 \\
\hline Medicago lupulina $\mathrm{L}$. & Fabaceae & $\mathrm{T}$ & WISP & & $\begin{array}{c}1,3,9,11,14,16,17,20,21,23,26,28 \\
39,40,42,45,46,53,55,60,63,65,69 \\
70\end{array}$ & $\begin{array}{l}\text { Ma-1964,1992; } \\
\text { V\&al-2019 }\end{array}$ \\
\hline Medicago minima (L.) Bartal. & Fabaceae & $\mathrm{T}$ & WISP & & $1,11,16,17,20,23,25,31,37,42,49$ & V\&al-2019 \\
\hline Medicago orbicularis (L.) Bartal. & Fabaceae & $\mathrm{T}$ & ME-circ & & 5 & \\
\hline Medicago polymorpha L. & Fabaceae & $\mathrm{T}$ & SEU-me & & 14 & Ma-1964 \\
\hline Medicago prostrata Jacq. & Fabaceae & $\mathrm{H}$ & SEU-me & & $\begin{array}{c}1,2,8,17,22,23,25,26,27,29,30,31 \\
32,34,43,44,45,47,48,51,56,58,59 \\
60,61,62,69,70\end{array}$ & $\begin{array}{l}\text { Tr-1992; LRS-1989; } \\
\text { V\&al-2019 }\end{array}$ \\
\hline Medicago rigidula (L.) All. & Fabaceae & $\mathrm{T}$ & ME-po & & $20,23,26,37,43$ & V\&al-2019 \\
\hline Medicago sativa L. ssp. sativa & Fabaceae & $\mathrm{H}$ & WISP & & \begin{tabular}{|c|}
$1,3,4,13,14,15,16,17,21,22,23,25$ \\
$27,32,37,38,42,43,46,48,50,53,54$ \\
$61,65,69,70$
\end{tabular} & $\begin{array}{l}\text { V\&al-2019 as M. sativa } \\
\text { L. }\end{array}$ \\
\hline Medicago truncatula Gaertn. & Fabaceae & $\mathrm{T}$ & ME-circ & & 16,23 & \\
\hline Melampyrum arvense $\mathrm{L}$. & Scrophulariaceae & $\mathrm{T}$ & EUAS & & Significant Landscape of Sutina & V\&al-2019 \\
\hline \begin{tabular}{|l|} 
Melampyrum barbatum Waldst. et \\
Kit. ssp. carstiense Ronniger
\end{tabular} & Scrophulariaceae & $\mathrm{T}$ & ME-ilape & & $17,49,70$ & \\
\hline Melampyrum cristatum L. & Scrophulariaceae & $\mathrm{T}$ & EUAS & & $18,25,65$ & Vi-1847:178 \\
\hline Melampyrum nemorosum $\mathrm{L}$. & Scrophulariaceae & $\mathrm{T}$ & EUAS & & 14 & Vi-1847:177 \\
\hline Melia azedarach L. & Meliaceae & $\mathrm{P}$ & CUAD & & 25 & \\
\hline Melica ciliata L. ssp. ciliata & Poaceae & $\mathrm{H}$ & EUAS & LC & $\begin{array}{c}1,3,5,6,9,11,13,14,17,21,22,23 \\
25,26,27,31,32,34,38,40,43,48,50 \\
53,55,56,58,65\end{array}$ & $\begin{array}{l}\text { LRS-1989 as } M . \\
\text { nebrodensis Parl.; } \\
\text { V\&al-2019 as M. ciliata } \\
\text { L. }\end{array}$ \\
\hline Melica nutans L. & Poaceae & $\mathrm{H}$ & EURO & & $14,32,35,38$ & \\
\hline Melica uniflora Retz. & Poaceae & $\mathrm{H}$ & EUAS & & Significant Landscape of Sutina & V\&al-2019 \\
\hline Melilotus albus Medik & Fabaceae & $\mathrm{T}$ & EUAS & & $13,46,58,59,65,66,69,70$ & V\&al-2019 \\
\hline Melilotus altissimus Thuill. & Fabaceae & G & EUAS & & 69 & \\
\hline Melilotus officinalis (L.) Lam. & Fabaceae & $\mathrm{H}$ & ME-east & & $\begin{array}{c}1,3,4,13,14,17,20,22,23,35,38,40 \\
45,46,53,55,59,61,62,66,69\end{array}$ & V\&al-2019 \\
\hline Melissa officinalis L & Lamiaceae & $\mathrm{H}$ & SEU-me & & $3,13,14,16,20,21,25,27,28,30,37$ & Vi-1847:201; V\&al-2019 \\
\hline Melittis melissophyllum L. & Lamiaceae & $\mathrm{H}$ & EURO & & $32,36,41$ & Vi-1847:212; V\&al-2019 \\
\hline Mentha aquatica $\mathrm{L}$. & Lamiaceae & $\mathrm{Hy}$ & WISP & & 22 & \\
\hline Mentha longifolia (L.) Huds. & Lamiaceae & $\mathrm{H}$ & WISP & & $\begin{array}{c}3,4,5,13,14,15,16,17,18,23,24,27 \\
28,37,42,48,50,51,58,65,68,69\end{array}$ & V\&al-2019 \\
\hline Mentha pulegium L. & Lamiaceae & $\mathrm{H}$ & EUAS & & 22 & \\
\hline Mentha spicata L. & Lamiaceae & $\mathrm{H}$ & WISP & & 42 & \\
\hline Mentha suaveolens Ehrh. & Lamiaceae & $\mathrm{H}$ & ME-circ & & 18 & \\
\hline Mentha x piperita L. & Lamiaceae & $\mathrm{H}$ & CUAD & & 66,66 & \\
\hline Mercurialis annua $\mathrm{L}$ & Euphorbiaceae & $\mathrm{T}$ & WISP & & $1,3,4,14,37,68$ & V\&al-2019 \\
\hline $\begin{array}{l}\text { Mercurialis ovata Sternb. et } \\
\text { Hoppe }\end{array}$ & Euphorbiaceae & G & SEU-po & & $11,12,30,65$ & L\&al-1987; V\&al-2019 \\
\hline
\end{tabular}




\begin{tabular}{|c|c|c|c|c|c|c|}
\hline Taxon & Family & \begin{tabular}{|c|} 
Life \\
form
\end{tabular} & Geoelement & Status & Localities (finding sites) & $\begin{array}{l}\text { Previously recorded } \\
\text { findings }\end{array}$ \\
\hline Mercurialis perennis L. & Euphorbiaceae & G & EURO & & $11,34,36,41,52,65$ & \\
\hline $\begin{array}{l}\text { Micromeria juliana (L.) Benth. ex } \\
\text { Rchb. }\end{array}$ & Lamiaceae & $\mathrm{Ch}$ & ME-circ & & $14,18,37,42,43$ & M\&K-2016; V\&al-2019 \\
\hline Minuartia capillacea (All.) Graebn. & Caryophyllaceae & Ch & ILBA-end & & 31 & L\&al-1987; LRS-1989 \\
\hline $\begin{array}{l}\text { Minuartia graminifolia (Ard.) Jav. } \\
\text { ssp. clandestina (Port.) Mattf. }\end{array}$ & Caryophyllaceae & $\mathrm{Ch}$ & ILBA-end & & Svilaja mountain range & $\begin{array}{l}\text { L\&R-1989 as M. } \\
\text { clandestina Port. }\end{array}$ \\
\hline $\begin{array}{l}\text { Minuartia mediterranea (Link.) K. } \\
\text { Malý }\end{array}$ & Caryophyllaceae & $\mathrm{T}$ & ME-circ & & 14 & \\
\hline $\begin{array}{l}\text { Minuartia verna (L.) Hiern ssp. } \\
\text { verna }\end{array}$ & Caryophyllaceae & $\mathrm{Ch}$ & ME-east & & 13 & \\
\hline Mirabilis jalapa L. & Nyctaginaceae & G & CUAD & & 22,27 & \\
\hline Misopates orontium (L.) Raf. & Scrophulariaceae & $\mathrm{T}$ & EUAS & & $4,13,24,28,49,66,68$ & \\
\hline Moehringia muscosa L. & Caryophyllaceae & $\mathrm{H}$ & SEU-mo & & $10,11,32,34,35,36,39,40$ & V\&al-2019 \\
\hline Moehringia trinervia (L.) Clairv. & Caryophyllaceae & $\mathrm{T}$ & EUAS & & $10,14,32$ & \\
\hline Moenchia mantica (L.) Bartl. & Caryophyllaceae & $\mathrm{T}$ & SEU-me & & 13,65 & \\
\hline Monotropa hypopitys L. & Pyrolaceae & G & WISP & & Significant Landscape of Sutina & V\&al-2019 \\
\hline Morus alba $\mathrm{L}$. & Moraceae & $\mathrm{P}$ & CUAD & & $\begin{array}{c}1,16,20,21,22,23,24,25,28,30^{*}, 31 \\
37,42,43^{*}, 59,69,70\end{array}$ & \\
\hline Morus nigra L. & Moraceae & $\mathrm{P}$ & CUAD & & $1,23^{*}, 70$ & \\
\hline Muscari botryoides (L.) Mill. & Asparagaceae & G & SEU-me & & $\begin{array}{c}5,10,11,12,30,34,36,41,44,47,51 \\
52,53,57,61,66,67,70\end{array}$ & $\begin{array}{l}\text { L\&R-1989; LRS-1989; } \\
\text { V\&al-2019 }\end{array}$ \\
\hline Muscari comosum (L.) Mill. & Asparagaceae & G & SEU-me & & $\begin{array}{c}6,13,14,19,20,23,37,41,42,43,44 \\
45,46,48,51,60,62,65,70\end{array}$ & V\&al-2019 \\
\hline Muscari neglectum Guss. ex Ten. & Asparagaceae & G & SEU-me & & $2,37,60,69$ & V\&al-2019 \\
\hline Myagrum perfoliatum $\mathrm{L}$. & Brassicaceae & $\mathrm{T}$ & EUAS & & 28 & \\
\hline Mycelis muralis (L.) Dumort. & Cichoriaceae & $\mathrm{H}$ & EUAS & & $11,14,32,34,38,39,40,63,65$ & V\&al-2019 \\
\hline Myosotis arvensis (L.) Hill & Boraginaceae & $\mathrm{T}$ & EUAS & & $17,25,46,55,69,70$ & V\&al-2019 \\
\hline $\begin{array}{l}\text { Myosotis ramosissima Rochel ssp. } \\
\text { ramosissima }\end{array}$ & Boraginaceae & $\mathrm{T}$ & WISP & DD & 37,44 & $\begin{array}{l}\text { V\&al-2019 as } M \text {. } \\
\text { ramosissima Rochel }\end{array}$ \\
\hline Myosotis suaveolens Willd. & Boraginaceae & $\mathrm{H}$ & EEUPO & $\begin{array}{c}\text { end, } \\
\text { sp }\end{array}$ & 35 & \\
\hline Myosotis sylvatica Hoffm. & Boraginaceae & $\mathrm{H}$ & EUAS & & 32,49 & \\
\hline Myosoton aquaticum (L.) Moench & Caryophyllaceae & $\mathrm{H}$ & EUAS & & $13,37,70$ & \\
\hline Myrrhoides nodosa (L.) Cannon & Apiaceae & $\mathrm{T}$ & ME-circ & & $3,16,20,25,29,37,42$ & \\
\hline Narcissus radiiflorus Salisb. & Amaryllidaceae & G & SEU-me & & $10,33,34,41,57$ & $\begin{array}{l}\text { Vi-1842:127 as N. } \\
\text { poeticus L.; L\&R-1989 as } \\
\text { N. angustifolius Curt.; } \\
\text { M\&al-2013; V\&al-2019 }\end{array}$ \\
\hline Neottia nidus-avis (L.) Rich. & Orchidaceae & G & EUAS & $\mathrm{sp}$ & 14 & $\begin{array}{l}\text { Kr-2005:136; Df-2006; } \\
\text { V\&al-2019 }\end{array}$ \\
\hline Nepeta cataria $\mathrm{L}$. & Lamiaceae & $\mathrm{H}$ & WISP & & $14,21,59$ & \\
\hline Nerium oleander L. & Apocynaceae & $\mathrm{P}$ & CUAD & & 1 & \\
\hline Nigella arvensis L. & Ranunculaceae & $\mathrm{T}$ & ME-east & & 4 & \\
\hline Nigella damascena $\mathrm{L}$. & Ranunculaceae & $\mathrm{T}$ & ME-circ & & $3,5,23,25,43$ & \\
\hline Odontites lutea (L.) Clairv & Scrophulariaceae & $\mathrm{T}$ & SEU-me & & $8,17,18,19,22,31,48$ & \\
\hline Odontites vulgaris Moench & Scrophulariaceae & $\mathrm{T}$ & SEU-me & & 4,22 & V\&al-2019 \\
\hline Oenanthe pimpinelloides L. & Apiaceae & $\mathrm{H}$ & ME-atl & & $22,23,46$ & V\&al-2019 \\
\hline Oenanthe silaifolia M. Bieb. & Apiaceae & $\mathrm{H}$ & SEU-po & & 28 & \\
\hline Oenothera speciosa Nuttall $^{*}$ & Onagraceae & $\mathrm{H}$ & CUAD & & 1 & \\
\hline Olea europaea L. & Oleaceae & $\mathrm{P}$ & CUAD & & $1,3,22 *, 25,43$ & \\
\hline $\begin{array}{l}\text { Onobrychis alba (Waldst. et Kit.) } \\
\text { Desv. ssp. alba }\end{array}$ & Fabaceae & $\mathrm{H}$ & ME-circ & & 16,59 & \\
\hline $\begin{array}{l}\text { Onobrychis arenaria (Kit.) DC. ssp. } \\
\text { arenaria }\end{array}$ & Fabaceae & $\mathrm{H}$ & EUAS & & 46 & \\
\hline $\begin{array}{l}\text { Onobrychis arenaria (Kit.) DC. ssp. } \\
\text { tommasinii (Jord.) Asch.et Graebn }\end{array}$ & Fabaceae & $\mathrm{H}$ & ME-ilade & $\begin{array}{c}\text { end, } \\
\mathrm{sp}\end{array}$ & $8,16,47,62$ & V\&al-2019 \\
\hline Onobrychis viciifolia Scop. & Fabaceae & $\mathrm{H}$ & EUAS & & Significant Landscape of Sutina & V\&al-2019 \\
\hline Ononis antiquorum (L.) Arcang. & Fabaceae & $\mathrm{Ch}$ & ME-circ & & $\begin{array}{c}7,8,13,15,22,23,26,28,32,38,39 \\
40,43,45,46,47 ?, 51,53,54,55,58 \\
59,60 ?, 62,64 ?, 65,69 ?\end{array}$ & \\
\hline Ononis pusilla L. & Fabaceae & $\mathrm{H}$ & SEU-me & & $21,26,43$ & Tr-1992 \\
\hline
\end{tabular}




\begin{tabular}{|c|c|c|c|c|c|c|}
\hline Taxon & Family & \begin{tabular}{|c|} 
Life \\
form
\end{tabular} & Geoelement & Status & Localities (finding sites) & $\begin{array}{c}\text { Previously recorded } \\
\text { findings }\end{array}$ \\
\hline Ononis spinosa $\mathrm{L}$. & Fabaceae & $\mathrm{H}$ & EURO & & $\begin{array}{c}15,22,23,28,35,47 ?, 50,57,60 ?, 61 \\
64 ?, 69 ?\end{array}$ & V\&al-2019 \\
\hline $\begin{array}{l}\text { Onopordum acanthium L. ssp. } \\
\text { acanthium }\end{array}$ & Asteraceae & $\mathrm{H}$ & EUAS & & $14,17,20,29,45,59$ & \\
\hline Onopordum illyricum L. & Asteraceae & $\mathrm{H}$ & ME-circ & & $1,2,3,5,6,21,23,25,27,43$ & \\
\hline $\begin{array}{l}\text { Onosma echioides (L.) L. ssp. } \\
\text { dalmatica (Scheele) Peruzziet N. } \\
\text { G. Passal }\end{array}$ & Boraginaceae & $\mathrm{Ch}$ & ME-ilape & $\begin{array}{l}\text { end, } \\
\text { sp }\end{array}$ & 58,61 & $\begin{array}{l}\text { Tr-1992 as O. javorkae } \\
\text { Simk.; V\&al-2019 }\end{array}$ \\
\hline Onosma stellulata Waldst. et Kit. & Boraginaceae & $\mathrm{Ch}$ & ME-ilade & $\begin{array}{l}\text { end, } \\
\text { sp }\end{array}$ & $41,64,69$ & Ja-1908; V\&al-2019 \\
\hline Onosma visianii Clementi & Boraginaceae & $\mathrm{H}$ & ILBA-end & & 57,62 & \\
\hline Ophrys apifera Huds. & Orchidaceae & G & SEU-me & EN, sp & 16,62 & $\begin{array}{l}\text { Kr-2005:144; Df-2006; } \\
\text { V\&al-2019 }\end{array}$ \\
\hline Ophrys bertolonii Moretti & Orchidaceae & G & ILBA-bap & $\begin{array}{l}\mathrm{VU}, \\
\mathrm{sp}\end{array}$ & Svilaja, Gradina & Kr-2005:162 \\
\hline $\begin{array}{l}\text { Ophrys dinarica Kranjčev et } \mathrm{P} \text {. } \\
\text { Delforge }\end{array}$ & Orchidaceae & G & ME-ilade & $\begin{array}{l}\text { end, } \\
\text { sp }\end{array}$ & 60 & Kr-2005:204; Df-2006 \\
\hline $\begin{array}{l}\text { Ophrys fuciflora (F.W.Schmidt) } \\
\text { Moench }\end{array}$ & Orchidaceae & G & SEU-me & $\begin{array}{l}\text { VU, } \\
\text { sp }\end{array}$ & $51,61,62$ & V\&al-2019 \\
\hline $\begin{array}{l}\text { Ophrys liburnica Devillers et } \\
\text { Devillers-Tersch. }\end{array}$ & Orchidaceae & G & ME-ilade & $\begin{array}{l}\text { end, } \\
\text { sp }\end{array}$ & $\begin{array}{c}\text { site } 269 \text { (XJ2650) 2,6 km N Potravlje, } \\
320 \mathrm{~m}\end{array}$ & Df-2006 \\
\hline $\begin{array}{l}\text { Ophrys rhodostephane Devillers et } \\
\text { Devillers-Tersch. }\end{array}$ & Orchidaceae & G & ME-ilade & $\begin{array}{l}\text { end, } \\
\text { sp }\end{array}$ & $\begin{array}{c}\text { site } 249 \text { ((XJ1261), } 1 \text { km S Vrlika, } 320 \\
\text { m }\end{array}$ & Df-2006 \\
\hline Ophrys scolopax Cav. & Orchidaceae & G & ME-circ & $\begin{array}{l}\text { DD, } \\
\mathrm{sp}\end{array}$ & Muć Gornji & Kr-2005:276 \\
\hline $\begin{array}{l}\text { Ophrys scolopax Cav. ssp. cornuta } \\
\text { (Steven) E. G. Camus }\end{array}$ & Orchidaceae & G & ME-po & $\begin{array}{l}\mathrm{DD} \\
\mathrm{sp}\end{array}$ & 14,17 & $\begin{array}{l}\text { Kr-2005:188 as O. } \\
\text { cornuta Steven; } \\
\text { V\&al-2019 } \\
\end{array}$ \\
\hline Ophrys sphegodes Mill. & Orchidaceae & G & ME-circ & $\begin{array}{l}\mathrm{VU}, \\
\mathrm{sp}\end{array}$ & 42 & Kr-2005:284 \\
\hline $\begin{array}{l}\text { Ophrys sphegodes Mill. ssp. atrata } \\
\text { (Rchb.f.) A.Bolòs }\end{array}$ & Orchidaceae & G & SEU-me & $\mathrm{sp}$ & Significant Landscape of Sutina & V\&al-2019 \\
\hline $\begin{array}{l}\text { Ophrys sphegodes Mill. ssp. } \\
\text { tommasinii (Vis.) Soó }\end{array}$ & Orchidaceae & G & ME-ilade & $\begin{array}{l}\text { end, } \\
\text { sp }\end{array}$ & 37,69 & \\
\hline Ophrys tetraloniae W.P.Teschner & Orchidaceae & G & ME-ilade & $\begin{array}{l}\text { end, } \\
\text { DD, } \\
\text { sp }\end{array}$ & 62 & Kr-2005:296; Df-2006 \\
\hline $\begin{array}{l}\text { Ophrys untchjii (M. Schulze) P. } \\
\text { Delforge }\end{array}$ & Orchidaceae & G & ME-ilade & $\begin{array}{l}\text { end, } \\
\text { sp }\end{array}$ & $\begin{array}{c}\text { site } 260 \text { (XJ1938) N Cimetiére de } \\
\text { Gornji Muć, 4560-470 m; site } 269 \\
\text { (XJ2650) 2,6 km N Potravlje, } 320 \text { m; }\end{array}$ & Df-2006 \\
\hline $\begin{array}{l}\text { Ophrys x kranjcevii P. Delforge } \\
\text { (O. dinarica } \times \text { O. untchjii) }\end{array}$ & Orchidaceae & G & ME-ilade & $\begin{array}{l}\text { end, } \\
\text { sp }\end{array}$ & $\begin{array}{c}\text { site } 278 \text { (XJ3742) Karakašica, 270-300 } \\
\text { m }\end{array}$ & Df-2006 \\
\hline Opopanax chironium (L.) Koch & Apiaceae & $\mathrm{H}$ & ME-circ & & 2,21 & V\&al-2019 \\
\hline Orchis coriophora L. ssp. coriophora & Orchidaceae & G & SEU-me & $\begin{array}{l}\mathrm{VU}, \\
\mathrm{sp}\end{array}$ & 46 & $\begin{array}{l}\text { Pe-1832:90, Kr-2005:310, } \\
\text { Df-2006 and V\&al-2019 } \\
\text { as } \\
\text { O. coriophora L. }\end{array}$ \\
\hline $\begin{array}{l}\text { Orchis coriophora L. ssp. fragrans } \\
\text { (Pollini) K. Richt. }\end{array}$ & Orchidaceae & G & ME-po & $\begin{array}{l}\text { VU, } \\
\mathrm{sp}\end{array}$ & $17,45,56$ & $\begin{array}{l}\text { Vi-1842:170; Kr-2005:314 } \\
\text { as O. fragrans Pollini }\end{array}$ \\
\hline Orchis laxiflora Lam. ssp. laxiflora & Orchidaceae & G & ME-atl & NT, sp & 16,28 & $\begin{array}{l}\text { Kr-2005:320 and Df-2006 } \\
\text { as } \\
\text { O. laxiflora Lam. }\end{array}$ \\
\hline Orchis mascula (L.) L. & Orchidaceae & G & ME-atl & NT, sp & Sutina-Bukovik, Mućko Zelovo & Kr-2005:324; Df-2006 \\
\hline Orchis militaris $\mathrm{L}$. & Orchidaceae & G & EUAS & $\begin{array}{l}\text { VU, } \\
\text { sp }\end{array}$ & 32 & $\begin{array}{l}\text { Vi-1842:169 as O. } \\
\text { variegata All.; } \\
\text { Kr-2005:326; Df-2006 }\end{array}$ \\
\hline Orchis morio L. & Orchidaceae & G & EUAS & NT, sp & $\begin{array}{c}7,8,13,14,18,32,41,42,51,52,57 \\
61,67\end{array}$ & $\begin{array}{l}\text { Kr-2005:328; Df-2006; } \\
\text { V\&al-2019 }\end{array}$ \\
\hline $\begin{array}{l}\text { Orchis morio L. ssp. picta (Loisel.) } \\
\text { K. Richt. }\end{array}$ & Orchidaceae & G & SEU-me & $\mathrm{sp}$ & $29,30,37,44,47,60,70$ & $\begin{array}{l}\text { Kr-2005:344 as O. picta } \\
\text { Loiseleur }\end{array}$ \\
\hline $\begin{array}{l}\text { Orchis provincialis Balb. ssp. } \\
\text { pauciflora (Ten.) Camus }\end{array}$ & Orchidaceae & G & ME-circ & $\begin{array}{l}\text { VU, } \\
\text { sp }\end{array}$ & $6,8,30,41,52$ & $\begin{array}{l}\text { Kr-2005:342 as } O \text {. } \\
\text { pauciflora Tenore; } \\
\text { V\&al-2019 }\end{array}$ \\
\hline
\end{tabular}




\begin{tabular}{|c|c|c|c|c|c|c|}
\hline Taxon & Family & \begin{tabular}{|c|} 
Life \\
form
\end{tabular} & Geoelement & Status & Localities (finding sites) & $\begin{array}{l}\text { Previously recorded } \\
\text { findings }\end{array}$ \\
\hline Orchis purpurea Huds. & Orchidaceae & G & EUAS & $\begin{array}{l}\text { VU, } \\
\text { sp }\end{array}$ & $37,42,45,47,60,61,69,70$ & Kr-2005:348; V\&al-2019 \\
\hline $\begin{array}{l}\text { Orchis quadripunctata Cirillo ex } \\
\text { Ten. }\end{array}$ & Orchidaceae & G & ME-east & $\begin{array}{l}\text { VU, } \\
\mathrm{sp}\end{array}$ & Svilaja, Bilopolje & Kr-2005:352 \\
\hline Orchis simia Lam. & Orchidaceae & G & ME-circ & $\begin{array}{l}\text { VU, } \\
\text { sp }\end{array}$ & Muć & Kr-2005:354 \\
\hline $\begin{array}{l}\text { Orchis tridentata Scop. ssp. } \\
\text { tridentata }\end{array}$ & Orchidaceae & G & ME-circ & $\begin{array}{l}\text { VU, } \\
\text { sp }\end{array}$ & $8,13,42,51,52,69$ & $\begin{array}{l}\text { Vi-1842:169 as } O . \\
\text { variegata All.; } \\
\text { Kr-2005:358, Df-2006 } \\
\text { and V\&al-2019 as O. } \\
\text { tridentata Scop. }\end{array}$ \\
\hline Origanum vulgare $\mathrm{L}$. & Lamiaceae & $\mathrm{H}$ & EUAS & & $4,5,11,13,15,19,32,35,38,67,70$ & V\&al-2019 \\
\hline Orlaya grandiflora $(\mathrm{L}$.$) Hoffm$ & Apiaceae & $\mathrm{T}$ & SEU-me & & $\begin{array}{c}1,3,5,6,9,13,16,17,18,19,21,23 \\
25,43,46\end{array}$ & V\&al-2019 \\
\hline Ornithogalum comosum $\mathrm{L}$. & Asparagaceae & G & SEEU & & $\begin{array}{c}8,11 ?, 17,23,37,41,42,45,47,52,56 \\
58\end{array}$ & Vi-1842:146; V\&al-2019 \\
\hline Ornithogalum gussonei Ten. & Asparagaceae & G & ME-circ & & $\begin{array}{c}\text { Svilaja mountain range; above the } \\
\text { village of Muć }\end{array}$ & L\&R-1989; Tr-1992 \\
\hline Ornithogalum pyramidale L. & Asparagaceae & G & SEU-me & & Significant Landscape of Sutina & V\&al-2019 \\
\hline Ornithogalum narbonense $\mathrm{L}$. & Asparagaceae & G & SEU-me & & 20,31 & VRZ-2012 \\
\hline $\begin{array}{l}\text { Ornithogalum refractum Kit. ex } \\
\text { Schltr. }\end{array}$ & Asparagaceae & G & SEU-me & & Significant Landscape of Sutina & VRZ-2013 \\
\hline Ornithogalum sibthorpii Greuter & Asparagaceae & G & SEEU & & $2,8,43,47$ & \\
\hline Ornithogalum umbellatum $\mathrm{L}$. & Asparagaceae & G & SEU-me & & Significant Landscape of Sutina & V\&al-2019 \\
\hline Orobanche alba Willd. & Orobanchaceae & G & EUAS & & Significant Landscape of Sutina & V\&al-2020 \\
\hline Orobanche gracilis Sm. & Orobanchaceae & G & SEU-me & & 11 & V\&al-2019 \\
\hline Orobanche minor Sm. & Orobanchaceae & $\mathrm{T}$ & SEU-me & & $31,34,41$ & $\begin{array}{l}\text { Tr-1992; VRZ-2012; } \\
\text { V\&al-2019 }\end{array}$ \\
\hline Orthilia secunda (L.) House & Pyrolaceae & $\mathrm{Ch}$ & $\mathrm{CIHO}$ & & $58(x=4844918, y=6382266)$ & Se-2016 \\
\hline Ostrya carpinifolia Scop. & Corylaceae & $\mathrm{P}$ & ME-ilseu & & $\begin{array}{c}1,9,10,11,14,17,18,19,21,30,31 \\
32,34,36,38,39,40,41,42,44,46,48 \\
51,56,57,58,59,64,66,69\end{array}$ & $\begin{array}{l}\text { Ja-1908; Je-1952; } \\
\text { Me-1972; L\&al-1987 as } \\
\text { O.c. ssp. corsica Rouy; } \\
\text { VRZ-2012; Mi\&al-2013; } \\
\text { V\&al-2019 }\end{array}$ \\
\hline Oxalis articulata Savigny & Oxalidaceae & G & CUAD & & 1,37 & \\
\hline Oxalis corniculata $\mathrm{L}$. & Oxalidaceae & $\mathrm{H}$ & WISP & & 14,27 & \\
\hline Oxalis dillenii Jacq. & Oxalidaceae & $\mathrm{H}$ & CUAD & & 4 & \\
\hline Oxytropis dinarica (Murb.) Wettst. & Fabaceae & $\mathrm{H}$ & ILBA-end & $\begin{array}{l}\text { end, } \\
\text { sp }\end{array}$ & 33 & $\begin{array}{l}\text { LRS-1989 as O. } \\
\text { campestris (L.) DC. ssp. } \\
\text { dinarica Murb. }\end{array}$ \\
\hline Paliurus spina-christi Mill. & Rhamnaceae & $\mathrm{P}$ & ME-ilseu & & $1,2,3,4,5,21,22,23,25,28,43,46$ & L\&al-1987; V\&al-2019 \\
\hline Panicum capillare $\mathrm{L}$. & Poaceae & $\mathrm{T}$ & CUAD & inv & 11,68 & \\
\hline Papaver dubium $\mathrm{L}$. & Papaveraceae & $\mathrm{T}$ & WISP & & "in herbidis demissis montis Svilaja" & Vi-1852:99 \\
\hline Papaver rhoeas L. & Papaveraceae & $\mathrm{T}$ & WISP & & $1,3,14,16,17,20,23,28,37,43,45,70$ & V\&al-2019 \\
\hline Papaver strigosum (Boenn.) Schur & Papaveraceae & $\mathrm{T}$ & ME-east & & $14,17,24,69$ & \\
\hline Parietaria judaica L. & Urticaceae & $\mathrm{H}$ & SEU-me & & $1,3,4,14,16,23,27,30,42,46,69$ & M\&K-2016; V\&al-2019 \\
\hline Parietaria officinalis L. & Urticaceae & $\mathrm{H}$ & SEU-me & & 14,69 & \\
\hline $\begin{array}{l}\text { Paronychia kapela (Hacq.) A. } \\
\text { Kerner }\end{array}$ & Caryophyllaceae & $\mathrm{H}$ & SEU-me & & $\begin{array}{c}2,6,8,9,10,11,17,26,31,33,34,38 \\
39,40,41,48,50,51,52,53,54,55,57 \\
58,61,62,65,69\end{array}$ & $\begin{array}{l}\text { L\&R-1989; VRZ-2012; } \\
\text { Se\&al-2016; V\&al-2019 }\end{array}$ \\
\hline $\begin{array}{l}\text { Parthenocissus quinquefolia (L.) } \\
\text { Planchon }\end{array}$ & Vitaceae & $\mathrm{P}$ & CUAD & inv & $1^{*}, 4,14,22,29,43^{*}, 59$ & \\
\hline $\begin{array}{l}\text { Pastinaca sativa L. ssp. urens (Req. } \\
\text { ex Godr.) Čelak. }\end{array}$ & Apiaceae & $\mathrm{H}$ & EUAS & & 59,59 & $\begin{array}{l}\text { Vi-1852:53 as P. sativa L. } \\
\text { B opaca }\end{array}$ \\
\hline $\begin{array}{l}\text { Paulownia tomentosa (Thunb.) } \\
\text { Steud. }\end{array}$ & Scrophulariaceae & $\mathrm{P}$ & CUAD & inv & $1,16^{*}, 22^{*}$ & \\
\hline $\begin{array}{l}\text { Pedicularis brachyodonta Schloss. } \\
\text { et Vuk. }\end{array}$ & Scrophulariaceae & $\mathrm{H}$ & ILBA-end & $\begin{array}{l}\text { end, } \\
\text { sp }\end{array}$ & Svilaja mountain range & LRS-1989 \\
\hline Pedicularis comosa L. & Scrophulariaceae & $\mathrm{H}$ & SEU-mo & LC & Veliki vrh and Debelo brdo & Ja-1908 \\
\hline Pedicularis friderici-augusti Tomm. & Scrophulariaceae & $\mathrm{H}$ & SEU-mo & $\begin{array}{c}\mathrm{DD}, \\
\mathrm{sp}\end{array}$ & 33 & Vi-1847:176 \\
\hline
\end{tabular}




\begin{tabular}{|c|c|c|c|c|c|c|}
\hline Taxon & Family & \begin{tabular}{|c|} 
Life \\
form
\end{tabular} & Geoelement & Status & Localities (finding sites) & $\begin{array}{l}\text { Previously recorded } \\
\text { findings }\end{array}$ \\
\hline Peltaria alliacea Jacq. & Brassicaceae & $\mathrm{H}$ & ME-ilseu & $\begin{array}{l}\text { end, } \\
\text { NT, sp }\end{array}$ & $10,32,33,35,39,40,41,47,52$ & Ja-1908 \\
\hline $\begin{array}{l}\text { Petrorhagia prolifera (L.) P. W. Ball } \\
\text { ex Heywood }\end{array}$ & Caryophyllaceae & $\mathrm{T}$ & EUAS & & $\begin{array}{c}1,3,7,8,13,14,19,21,25,27,28,35 \\
43,46,48,49,55,58\end{array}$ & V\&al-2019 \\
\hline Petrorhagia saxifraga (L.) Link & Caryophyllaceae & $\mathrm{H}$ & SEU-me & & $\begin{array}{c}1,3,4,5,6,7,8,11,13,15,17,18,19 \\
21,22,23,25,26,27,29,31,35,37,40 \\
42,43,45,46,48,49,50,53,54,55,58 \\
59,65,66,70\end{array}$ & $\begin{array}{l}\text { Tr-1992; VRZ-2012; } \\
\text { V\&al-2019 }\end{array}$ \\
\hline $\begin{array}{l}\text { Petteria ramentacea (Sieber) C. } \\
\text { Presl }\end{array}$ & Fabaceae & $P$ & ME-ilade & & $\begin{array}{c}\text { on the southern dolomite slopes of } \\
\text { Svilaja towards Muć }\end{array}$ & L\&al-1987 \\
\hline Peucedanum carvifolia Vill. & Apiaceae & $\mathrm{H}$ & SEU-po & & 22,70 & \\
\hline Peucedanum cervaria (L.) Lapeyr & Apiaceae & $\mathrm{H}$ & SEU-me & & 19,31 & \\
\hline Peucedanum coriaceum Rchb. & Apiaceae & $\mathrm{H}$ & ME-ilade & $\begin{array}{l}\text { end, } \\
\text { DD, } \\
\text { sp }\end{array}$ & 22 & $\begin{array}{l}\text { Vi-1852:51 as } P \text {. petteri } \\
\text { Vis. }\end{array}$ \\
\hline $\begin{array}{l}\text { Peucedanum longifolium Waldst. } \\
\text { et Kit. }\end{array}$ & Apiaceae & $\mathrm{H}$ & ILBA-end & & "in rupestribus montium Svilaja" & Vi-1852:51 \\
\hline $\begin{array}{l}\text { Peucedanum oreoselinum (L.) } \\
\text { Moench }\end{array}$ & Apiaceae & $\mathrm{H}$ & EURO & & $15,64,69,70$ & Vi-1852:52; V\&al-2019 \\
\hline $\begin{array}{l}\text { Phleum pratense L. ssp. bertolonii } \\
\text { (DC.) Bornm. }\end{array}$ & Poaceae & $\mathrm{H}$ & EUAS & & $13,17,22,24,25,28,43$ & V\&al-2019 \\
\hline $\begin{array}{l}\text { Phleum subulatum (Savi) Asch. et } \\
\text { Graebn. }\end{array}$ & Poaceae & $\mathrm{T}$ & ME-circ & & $3,4,5,23$ & \\
\hline $\begin{array}{l}\text { Phragmites australis (Cav.) Trin. } \\
\text { ex Steud. }\end{array}$ & Poaceae & G & WISP & & 28,70 & \\
\hline Physalis alkekengi $\mathrm{L}$. & Solanaceae & $\mathrm{H}$ & EUAS & & 18,70 & \\
\hline $\begin{array}{l}\text { Physospermum verticillatum } \\
\text { (Waldst. et Kit.) Vis. }\end{array}$ & Apiaceae & $\mathrm{H}$ & ME-circ & & $\begin{array}{c}\text { "in rupestribus elatioribus et } \\
\text { sylvaticis montis Promina et Svilaja" }\end{array}$ & $\begin{array}{l}\text { Vi-1852:69 as Alschingera } \\
\text { verticillata Vis. }\end{array}$ \\
\hline Phyteuma orbiculare L. & Campanulaceae & $\mathrm{H}$ & EUAS & & 33,34 & V\&al-2019 \\
\hline Picnomon acarna (L.) Cass. & Asteraceae & $\mathrm{H}$ & ME-circ & & $6,21,23,25,27,30,43$ & \\
\hline Picris echioides L. & Cichoriaceae & $\mathrm{T}$ & ME-circ & & $1,4,22,68$ & V\&al-2019 \\
\hline $\begin{array}{l}\text { Picris hieracioides L. ssp. } \\
\text { hieracioides }\end{array}$ & Cichoriaceae & $\mathrm{H}$ & EUAS & & $\begin{array}{c}4,8,13,14,15,18,19,26,27,32,34 \\
35,40,45,55,59,61,63,65\end{array}$ & $\begin{array}{l}\text { V\&al-2019 as } P \text {. } \\
\text { hieracioides L. }\end{array}$ \\
\hline $\begin{array}{l}\text { Picris hieracioides L. ssp. spinulosa } \\
\text { (Bertol. ex Guss.) Arcang. }\end{array}$ & Cichoriaceae & $\mathrm{H}$ & SEU-me & & $1,4,15,22,25,43,46,47,48,60$ & \\
\hline Picris hispidissima (Bartl.) Koch & Cichoriaceae & $\mathrm{H}$ & ME-ilade & & $6,23,38,55,58,62$ & V\&al-2019 \\
\hline Pimpinella saxifraga L. & Apiaceae & $\mathrm{H}$ & EUAS & & $7,13,15,23$ & V\&al-2019 \\
\hline Pinus nigra Arnold. & Pinaceae & $P$ & SEU-me & & $\begin{array}{c}1,6,7,8,9,28,37,39,41,43,44,45 \\
46,47,50^{*}, 51,52,53,55,56,57,58,59 \\
60,61,62,64,66,69\end{array}$ & $\begin{array}{l}\text { A-1929:48; Je-1952; } \\
\text { L\&al-1987 as P. n. var } \\
\text { intermedia H-ić; } \\
\text { V\&al-2019 }\end{array}$ \\
\hline Pinus pinaster Aiton & Pinaceae & $\mathrm{P}$ & ME-atl & & 37,64 & Me-1972 \\
\hline Piptatherum miliaceum (L.) Coss. & Poaceae & $\mathrm{H}$ & ME-circ & & 35 & \\
\hline Pistacia lentiscus L. & Anacardiaceae & $P$ & ME-circ & & Significant Landscape of Sutina & V\&al-2019 \\
\hline Pistacia terebinthus L. & Anacardiaceae & $\mathrm{P}$ & ME-circ & & 14 & M\&K-2016 \\
\hline Plantago altissima L. & Plantaginaceae & $\mathrm{H}$ & SEU-me & & $\begin{array}{c}3,4,5,14,15,16,17,20,27,28,36,37 \\
42,43,46,58,60,65,70\end{array}$ & \\
\hline Plantago argentea Chaix & Plantaginaceae & $\mathrm{H}$ & SEU-mo & & $32,34,51,52,53,58,59$ & Vi-1847:3; LRS-1989 \\
\hline Plantago holosteum Scop. & Plantaginaceae & $\mathrm{H}$ & ME-circ & LC & $\begin{array}{c}6,7,8,9,11,13,23,26,31,33,34,35 \\
38,39,40,43,47,50,51,52,53,54,55 \\
56,57,58,59,61,62,64,65,69\end{array}$ & $\begin{array}{l}\text { Je-1952; Tr-1992 as P.h. } \\
\text { Scop. ssp. holosteum and } \\
\text { P. h. Scop. ssp. } \\
\text { depauperata (Godr.) H-ić; } \\
\text { VRZ-2012; V\&al-2019 }\end{array}$ \\
\hline Plantago lanceolata $\mathrm{L}$. & Plantaginaceae & $\mathrm{H}$ & WISP & & $\begin{array}{c}1,2,3,4,10,13,14,15,17,18,19,27 \\
29,31,36,45,47,48,50,51,57,59,60 \\
61,66,68\end{array}$ & $\begin{array}{l}\text { Ma-1964,1992; } \\
\text { V\&al-2019 }\end{array}$ \\
\hline $\begin{array}{l}\text { Plantago major L. ssp. intermedia } \\
\text { (Gilib.) Lange }\end{array}$ & Plantaginaceae & $\mathrm{H}$ & WISP & & $\begin{array}{c}2,11,14,15,16,17,19,20,22,23,27 \\
28,35,36,37,38,48,51,59,63,66,69 \\
70\end{array}$ & Ma-1964 \\
\hline Plantago major L. ssp. major & Plantaginaceae & $\mathrm{H}$ & WISP & & 4,7 & V\&al-2019 as P. major L. \\
\hline
\end{tabular}




\begin{tabular}{|c|c|c|c|c|c|c|}
\hline Taxon & Family & $\begin{array}{c}\text { Life } \\
\text { form }\end{array}$ & Geoelement & Status & Localities (finding sites) & $\begin{array}{l}\text { Previously recorded } \\
\text { findings }\end{array}$ \\
\hline Plantago media $\mathrm{L}$. & Plantaginaceae & $\mathrm{H}$ & EUAS & & $\begin{array}{c}2,7,8,10,11,15,16,17,18,19,21,26 \\
27,31,34,37,42,44,45,46,47,48,50 \\
58,60,61,62,64,65,66,67,69,70\end{array}$ & Je-1952; V\&al-2019 \\
\hline Platanthera bifolia (L.) Rich. & Orchidaceae & G & EUAS & $\begin{array}{l}\text { VU, } \\
\mathrm{sp}\end{array}$ & Muć; plateau of Svilaja & $\begin{array}{l}\text { Kr-2005:364; Df-2006; } \\
\text { Se-2016 }\end{array}$ \\
\hline $\begin{array}{l}\text { Platanthera chlorantha (Custer) } \\
\text { Rchb. }\end{array}$ & Orchidaceae & G & EUAS & NT, sp & 51,67 & Kr-2005:366; Df-2006 \\
\hline Platanus $x$ acerifolia (Aiton) Willd. & Platanaceae & $\mathrm{P}$ & CUAD & & $14,22^{*}$ & \\
\hline Plumbago europaea L. & Plumbaginaceae & $\mathrm{Ch}$ & ME-circ & & $1,3,21,23,25,27$ & \\
\hline Poa annua L. & Poaceae & $\mathrm{T}$ & WISP & LC & $2,16,61,69$ & Ma-1964; V\&al-2019 \\
\hline Poa bulbosa $\mathrm{L}$. & Poaceae & $\mathrm{H}$ & EUAS & & $\begin{array}{l}2,8,9,11,17,20,29,30,32,35,36,37 \\
38,39,40,42,44,47,55,58,61,69,70\end{array}$ & V\&al-2019 \\
\hline Poa compressa $\mathrm{L}$ & Poaceae & $\mathrm{H}$ & WISP & & $11,25,27,35,55$ & Vi-1842:80 \\
\hline Poa nemoralis L. & Poaceae & $\mathrm{H}$ & $\mathrm{CIHO}$ & & 11,37 & \\
\hline Poa pratensis L. & Poaceae & $\mathrm{H}$ & WISP & & $11,32,36,37,61,63$ & \\
\hline $\begin{array}{l}\text { Poa trivialis L. ssp. sylvicola } \\
\text { (Guss.) H.Lindb. }\end{array}$ & Poaceae & $\mathrm{H}$ & ME-eu & LC & $\begin{array}{c}14,28,29,37,44,46,47,48,60,63,64 \\
69\end{array}$ & $\begin{array}{l}\text { Ma-1992 as P. sylvicola } \\
\text { Guss. }\end{array}$ \\
\hline Poa trivialis L. ssp. trivialis & Poaceae & $\mathrm{H}$ & EUAS & & 70 & \\
\hline Polycnemum arvense L. & Chenopodiaceae & $\mathrm{T}$ & EUAS & $\begin{array}{l}\text { DD, } \\
\text { sp }\end{array}$ & 65 & \\
\hline $\begin{array}{l}\text { Polygala nicaeensis Risso ex Koch } \\
\text { ssp. mediterranea Chodat }\end{array}$ & Polygalaceae & $\mathrm{H}$ & ME-circ & & $2,24,25,26,29,57,61,62,70$ & V\&al-2019 \\
\hline Polygala vulgaris $\mathrm{L}$. & Polygalaceae & $\mathrm{H}$ & EUAS & & $12,13,14,29,37,44,47,60,64,67,69$ & \\
\hline $\begin{array}{l}\text { Polygonatum latifolium (Jacq.) } \\
\text { Desf }\end{array}$ & Asparagaceae & G & EEUPO & $\begin{array}{l}\mathrm{VU}, \\
\mathrm{sp}\end{array}$ & 32 & $\begin{array}{l}\text { Vi-1842:163 as } \\
\text { Convallaria latifolia Jacq. }\end{array}$ \\
\hline Polygonatum multiflorum (L.) All & Asparagaceae & G & $\mathrm{CIHO}$ & & 40,32 & $\begin{array}{l}\text { Vi-1842:163 as } \\
\text { Convallaria multiflora L. }\end{array}$ \\
\hline $\begin{array}{l}\text { Polygonatum odoratum (Mill.) } \\
\text { Druce }\end{array}$ & Asparagaceae & G & EUAS & & $36,40,41,52$ & $\begin{array}{l}\text { Vi-1842:162 as } \\
\text { Convallaria polygonatum } \\
\text { L. }\end{array}$ \\
\hline Polygonum arenastrum Boreau & Polygonaceae & $\mathrm{T}$ & WISP & & $4,13,14,15,27,49,50$ & \\
\hline Polygonum aviculare $\mathrm{L}$. & Polygonaceae & $\mathrm{T}$ & WISP & & $\begin{array}{c}3,4,5,6,8,11,13,15,16,24,27,49 \\
50,59,66\end{array}$ & Ma-1964 \\
\hline Polygonum lapathifolium L. & Polygonaceae & $\mathrm{T}$ & WISP & & 14,22 & \\
\hline Polygonum persicaria $\mathrm{L}$. & Polygonaceae & $\mathrm{T}$ & WISP & & $4,16,28,49$ & \\
\hline Polypodium cambricum $\mathrm{L}$. & Polypodiaceae & $\mathrm{H}$ & SEU-me & & 14,31 & VRZ-2012; V\&al-2019 \\
\hline Polypodium vulgare $\mathrm{L}$. & Polypodiaceae & $\mathrm{H}$ & WISP & & "in umbrosis Svilaja" & Vi-1842:36 \\
\hline Populus alba L. & Salicaceae & $\mathrm{P}$ & EUAS & & 43 & $\begin{array}{l}\text { Pe-1832; Vi-1842:214; } \\
\text { L\&al-1987 }\end{array}$ \\
\hline Populus nigra $\mathrm{L}$. & Salicaceae & $P$ & EUAS & & $\begin{array}{c}1,4,5,14,15,16,28,32,43,44,46,47 \\
61,70\end{array}$ & V\&al-2019 \\
\hline Populus tremula $\mathrm{L}$. & Salicaceae & $\mathrm{P}$ & EUAS & & $14,32,34,35,41,42,66$ & Vi-1842:214; V\&al-2019 \\
\hline Portulaca oleracea L. & Portulacaceae & $\mathrm{T}$ & WISP & & $1,3,4,5,14,15,22,27,49,50,65,68$ & \\
\hline Potamogeton natans L. & $\begin{array}{c}\text { Potamo- } \\
\text { getonaceae }\end{array}$ & Hy & WISP & & 22,48 & \\
\hline Potentilla argentea $\mathrm{L}$. & Rosaceae & $\mathrm{H}$ & WISP & & 13,37 & Tr-1992 \\
\hline Potentilla australis Krašan & Rosaceae & $\mathrm{H}$ & ME-ilade & & $\begin{array}{c}2,6,10,11,12,30,36,39,41,44,50 \\
51,52,56,57,61,62,64,67,69\end{array}$ & V\&al-2019 \\
\hline Potentilla cinerea Chaix ex Vill. & Rosaceae & $\mathrm{H}$ & EURO & & 32,35 & $\begin{array}{l}\text { Vi-1852:252 as } P \text {. } \\
\text { subacaulis L. }\end{array}$ \\
\hline Potentilla detommasii Ten. & Rosaceae & $\mathrm{H}$ & SEEU & & 25,40 & \\
\hline Potentilla erecta (L.) Raeuschel & Rosaceae & $\mathrm{H}$ & EUAS & & 61,63 & Je-1952 \\
\hline Potentilla hirta L. & Rosaceae & $\mathrm{H}$ & SEU-me & & $1,2,17,26,29$ & V\&al-2019 \\
\hline $\begin{array}{l}\text { Potentilla micrantha Ramond ex } \\
\text { DC. }\end{array}$ & Rosaceae & $\mathrm{H}$ & SEU-po & & $10,12,36$ & V\&al-2019 \\
\hline Potentilla recta $\mathrm{L}$. & Rosaceae & $\mathrm{H}$ & EUAS & & $5,17,25,29,59,61$ & \\
\hline Potentilla reptans $\mathrm{L}$. & Rosaceae & $\mathrm{H}$ & WISP & & $\begin{array}{c}1,2,5,13,14,16,17,20,22,27,28,43 \\
44,45,46,50,61,70\end{array}$ & V\&al-2019 \\
\hline Prenanthes purpurea $\mathrm{L}$. & Cichoriaceae & $\mathrm{H}$ & CEU & & Significant Landscape of Sutina & V\&al-2019 \\
\hline $\begin{array}{l}\text { Primula veris L. ssp. columnae } \\
\text { (Ten.) Lüdi }\end{array}$ & Primulaceae & $\mathrm{H}$ & SEU-mo & NT & $10,11,27,32,34,36,40,41,53,66,67$ & V\&al-2019 \\
\hline
\end{tabular}




\begin{tabular}{|c|c|c|c|c|c|c|}
\hline Taxon & Family & \begin{tabular}{|c|} 
Life \\
form
\end{tabular} & Geoelement & Status & Localities (finding sites) & $\begin{array}{l}\text { Previously recorded } \\
\text { findings }\end{array}$ \\
\hline Primula vulgaris Huds. & Primulaceae & $\mathrm{H}$ & SEU-atl & & $14,19,42,63,65$ & Je-1952; V\&al-2019 \\
\hline Prunella laciniata (L.) L. & Lamiaceae & $\mathrm{H}$ & SEU-me & & $8,25,27,32,34,35,38,39,40,65$ & V\&al-2019 \\
\hline Prunella vulgaris L. & Lamiaceae & $\mathrm{H}$ & WISP & & $19,46,59$ & V\&al-2019 \\
\hline Prunus avium $\mathrm{L}$. & Rosaceae & $\mathrm{P}$ & CUAD & & $\begin{array}{c}1^{*}, 3^{*}, 13,14,16^{*}, 20,21,23,25,27,28, \\
30,37,42,43^{*}, 45,46^{*}, 47,48,49,50, \\
55,59,65,66^{*}, 69^{*}, 70\end{array}$ & V\&al-2019 \\
\hline Prunus cerasifera Ehrh. & Rosaceae & $P$ & CUAD & & $\begin{array}{c}1,4,14,15,17,21,27,30,37^{*}, 42,43 \\
50,55,65,69^{*}\end{array}$ & \\
\hline Prunus cerasus L. & Rosaceae & $\mathrm{P}$ & CUAD & & $\begin{array}{c}1^{*}, 3^{*}, 4^{*}, 16^{*}, 21^{*}, 23^{*}, 27^{*}, 28,37^{*}, \\
46^{*}, 66^{*}, 69,70^{*}\end{array}$ & \\
\hline Prunus domestica $\mathrm{L}$. & Rosaceae & $\mathrm{P}$ & CUAD & & $\begin{array}{c}1,11,13^{*}, 14,16,20,21,23,28,29,37^{*} \\
42,43^{*}, 44,47,49^{*}, 58,61^{*}, 65,66^{*} \\
69^{*}, 70\end{array}$ & \\
\hline Prunus dulcis (Mill.) D. A. Webb. & Rosaceae & $\mathrm{P}$ & CUAD & & $1,4,5,14,23,25,27,43^{*}, 49^{*}, 70$ & \\
\hline Prunus mahaleb $\mathrm{L}$. & Rosaceae & $\mathrm{P}$ & SEU-me & & $\begin{array}{c}1,3,4,5,6,8,9,11,13,21,23,25,26 \\
27,31,32,33,34,35,36,37,38,39,40 \\
41,42,43,44,45,46,47,48,49,50,51 \\
52,53,54,55,56,57,58,59,61,62,64 \\
65\end{array}$ & $\begin{array}{l}\text { Je-1952; L\&al-1987 as } \\
\text { Cerasus cupaniana (Ten.) } \\
\text { M.G.; VRZ-2012; } \\
\text { Se\&al-2016; V\&al-2019 }\end{array}$ \\
\hline Prunus persica (L.) Batsch & Rosaceae & $\mathrm{P}$ & CUAD & & $3^{*}, 43^{*}, 65$ & \\
\hline Prunus spinosa $\mathrm{L}$. & Rosaceae & P & EUAS & & \begin{tabular}{|}
$2,4,5,7,13,14,15,16,17,18,19,20$, \\
$21,22,26,27,28,29,34,39,41,42,43$ \\
$44,45,46,47,48,50,54,55,58,59,61$ \\
$62,64,65,69,70$
\end{tabular} & L\&al-1987; V\&al-2019 \\
\hline $\begin{array}{l}\text { Pseudolysimachion barrelieri } \\
\text { (Schott ex Roem. et Schult.) } \\
\text { Holub }\end{array}$ & Scrophulariaceae & $\mathrm{H}$ & SEEU & & $\begin{array}{c}6,7,8,10,17,19,26,34,39,40,48,51 \\
53,55,57,58,67\end{array}$ & V\&al-2019 \\
\hline $\begin{array}{l}\text { Pteridium aquilinum (L.) Kuhn } \\
\text { ssp. aquilinum }\end{array}$ & Hypolepidaceae & G & WISP & & $37,40,65,70$ & $\begin{array}{l}\text { Je-1952; L\&al-1987 as } P . \\
\text { tauricum Grossh.; } \\
\text { V\&al-2019 }\end{array}$ \\
\hline Pulicaria dysenterica (L.) Bernh. & Asteraceae & $\mathrm{H}$ & SEU-me & & 4,22 & Vi-1847:65 \\
\hline Pulmonaria angustifolia L. & Boraginaceae & $\mathrm{H}$ & SEU-po & & 10,11 & \\
\hline Pulsatilla grandis Wender. & Ranunculaceae & $\mathrm{H}$ & EEUPO & $\mathrm{LC}, \mathrm{sp}$ & $10,12,41,53,67$ & $\begin{array}{l}\text { M\&al-2013; Se-2016; } \\
\text { V\&al-2019 }\end{array}$ \\
\hline Punica granatum $\mathrm{L}$. & Punicaceae & $\mathrm{P}$ & CUAD & & $1,22^{*}, 37^{*}, 43^{*}, 49^{*}, 70$ & \\
\hline Pyracantha coccinea M. J. Roemer & Rosaceae & $\mathrm{P}$ & CUAD & & 50 & \\
\hline Pyrola minor $\mathrm{L}$. & Pyrolaceae & $\mathrm{H}$ & $\mathrm{CIHO}$ & & Significant Landscape of Sutina & V\&al-2019 \\
\hline Pyrus amygdaliformis Vill. & Rosaceae & $\mathrm{P}$ & SEU-me & & $\begin{array}{c}1,5,6,13,21,27,44,45,47,48,49,51 \\
55,61\end{array}$ & $\begin{array}{l}\text { Je-1952; L\&al-1987 as } P . \\
\text { spinosa Forssk.; } \\
\text { Se\&al-2016; V\&al-2019 }\end{array}$ \\
\hline Pyrus communis L. & Rosaceae & $\mathrm{P}$ & CUAD & & $1^{*}, 3^{*}, 20,37^{*}, 44,66^{*}, 69^{*}, 70^{*}$ & Vi-1852:244 \\
\hline Pyrus pyraster Burgsd. & Rosaceae & $\mathrm{P}$ & EUAS & & $7,8,11,13,16,17,31,35,36,41,43,60$ & \\
\hline Quercus cerris L. & Fagaceae & $\mathrm{P}$ & SEU-po & & $13,14,15,16,17,18,19,20,48,61$ & Vi-1842:208; V\&al-2019 \\
\hline $\begin{array}{l}\text { Quercus pubescens Willd. (incl. } \\
\text { Quercus virgiliana (Ten.) Ten.) }\end{array}$ & Fagaceae & $\mathrm{P}$ & SEU-po & & $\begin{array}{c}1,2,3,4,5,6,17,18,19,21,23,24,25 \\
26,27,29,30,31,36,37,41,42,43,44 \\
45,46,47,49,50,51,52,55,57,58,59 \\
\quad 60,61,62,64,65,66,67,70\end{array}$ & $\begin{array}{l}\text { Je-1952 i Me-1972 as Q. } \\
\text { lanuginosa Thuill.; } \\
\text { L\&al-1987 as Q. } \\
\text { brachyphylla Kotschy; } \\
\text { VRZ-2012; V\&al-2019 }\end{array}$ \\
\hline Ranunculus acris L. & Ranunculaceae & $\mathrm{H}$ & WISP & & $14,28,42,46,65,70$ & V\&al-2019 \\
\hline Ranunculus arvensis L. & Ranunculaceae & $\mathrm{T}$ & EURO & & 67 & \\
\hline Ranunculus bulbosus L. & Ranunculaceae & $\mathrm{H}$ & EUAS & & $11,29,30,37,47,48,58,61,65,70$ & V\&al-2019 \\
\hline Ranunculus carinthiacus Hoppe & Ranunculaceae & $\mathrm{H}$ & ME-circ & & 32,35 & $\begin{array}{l}\text { Vi-1852:85 as } R \text {. } \\
\text { montanus W. } \mathbf{0} \\
\text { tenuifolius; Ja-1908 }\end{array}$ \\
\hline $\begin{array}{l}\text { Ranunculus ficaria L. ssp. } \\
\text { calthifolius (Rchb.) Arcang. }\end{array}$ & Ranunculaceae & G & SEU-me & & $2,5,6,10,11,36,41,52,53,57,66^{*}, 67$ & V\&al-2019 as R. ficaria L. \\
\hline Ranunculus illyricus L. & Ranunculaceae & $\mathrm{H}$ & SEU-co & & $8,11,12,13,14,29,37,51,52,57,67$ & Se\&al-2016 \\
\hline Ranunculus lanuginosus L. & Ranunculaceae & $\mathrm{H}$ & CEU & & 14,63 & \\
\hline Ranunculus millefoliatus Vahl. & Ranunculaceae & $\mathrm{H}$ & SEU-me & & $2,10,11,12,29,37,44,47,57,61,67$ & V\&al-2019 \\
\hline Ranunculus neapolitanus L. & Ranunculaceae & $\mathrm{H}$ & SEU-me & & $\begin{array}{c}6,11,14,16,20,21,25,37,42,45,46 \\
47,60,61,63,69\end{array}$ & \\
\hline
\end{tabular}




\begin{tabular}{|c|c|c|c|c|c|c|}
\hline Taxon & Family & $\begin{array}{c}\text { Life } \\
\text { form }\end{array}$ & Geoelement & Status & Localities (finding sites) & $\begin{array}{c}\text { Previously recorded } \\
\text { findings }\end{array}$ \\
\hline Ranunculus ophioglossifolius Vill. & Ranunculaceae & $\mathrm{H}$ & SEU-me & EN, sp & 28 & \\
\hline Ranunculus polyanthemos L. & Ranunculaceae & $\mathrm{H}$ & EUAS & & 51,52 & \\
\hline Ranunculus repens L. & Ranunculaceae & $\mathrm{H}$ & WISP & & 23,70 & \\
\hline Ranunculus sardous Crantz & Ranunculaceae & $\mathrm{T}$ & WISP & & $22,23,46$ & $\begin{array}{l}\text { Vi-1852:86 as } R \text {. } \\
\text { philonotis Ehrh. }\end{array}$ \\
\hline Ranunculus velutinus Ten. & Ranunculaceae & $\mathrm{H}$ & ILBA-bap & & 64 & V\&al-2019 \\
\hline $\begin{array}{l}\text { Rapistrum rugosum (L.) All. ssp. } \\
\text { rugosum }\end{array}$ & Brassicaceae & $\mathrm{T}$ & ME-circ & & 3 & \\
\hline Reseda lutea L. & Resedaceae & $\mathrm{H}$ & WISP & & $1,3,14,16,25,29,44,45,69,70$ & V\&al-2019 \\
\hline Reseda phyteuma L & Resedaceae & $\mathrm{T}$ & SEU-me & & $4,27,29,68,69$ & \\
\hline Reynoutria japonica Houtt. & Polygonaceae & G & CUAD & inv & 28 & \\
\hline Rhamnus alaternus L. & Rhamnaceae & $\mathrm{P}$ & ME-circ & & 31 & \\
\hline $\begin{array}{l}\text { Rhamnus alpinus L. ssp. fallax } \\
\text { (Boiss.) Maire et Petitm. }\end{array}$ & Rhamnaceae & $\mathrm{P}$ & ILBA-end & & $9,11,32,33,34,35,36,52$ & Ja-1908 as R. falax \\
\hline Rhamnus cathartica $\mathrm{L}$. & Rhamnaceae & $\mathrm{P}$ & EUAS & & Significant Landscape of Sutina & V\&al-2019 \\
\hline $\begin{array}{l}\text { Rhamnus intermedius Steud. et } \\
\text { Hochst }\end{array}$ & Rhamnaceae & $\mathrm{P}$ & ME-ilade & $\begin{array}{c}\text { end, } \\
\text { NT, } \\
\text { sp }\end{array}$ & $\begin{array}{c}1,2,5,6,8,9,11,13,14,21,23,25,26 \\
27,31,34,39,43,48,52,53,56,57,58 \\
61\end{array}$ & VRZ-2012; V\&al-2019 \\
\hline Rhamnus saxatilis Jacq. & Rhamnaceae & $\mathrm{P}$ & EUAS & LC & $6,7,10,30,31,47,59$ & \\
\hline Rhinanthus minor L. & Scrophulariaceae & $\mathrm{T}$ & $\mathrm{CIHO}$ & & $7,16,28,45,53,55,58,59,65,70$ & V\&al-2019 \\
\hline Robinia pseudoacacia L. & Fabaceae & $P$ & CUAD & inv & $\begin{array}{c}1,3,5,7,13,14,16,17,22,24,29,37 \\
44,45,46,47,50,55,57,58,59,60,61 \\
62,63,64,69,70\end{array}$ & V\&al-2019 \\
\hline Rorippa lippizensis (Wulfen) Rchb. & Brassicaceae & $\mathrm{H}$ & ILBA-end & $\begin{array}{c}\text { end, } \\
\text { sp }\end{array}$ & $11,13,14,20,28,29,30,44,70$ & V\&al-2019 \\
\hline Rorippa sylvestris (L.) Besser & Brassicaceae & $\mathrm{H}$ & EUAS & & $1,22,23,28$ & V\&al-2019 \\
\hline Rosa arvensis Huds. & Rosaceae & $\mathrm{P}$ & CEU & & Significant Landscape of Sutina & V\&al-2019 \\
\hline Rosa canina $\mathrm{L}$. & Rosaceae & $\mathrm{P}$ & WISP & & $1,17,19,30,35,42,47,59,64$ & V\&al-2019 \\
\hline Rosa glauca Pourr. & Rosaceae & $\mathrm{P}$ & EURO & & $20,32,34,35,36,40$ & \\
\hline Rosa pimpinellifolia $\mathrm{L}$. & Rosaceae & $\mathrm{P}$ & EUAS & & 17,32 & \\
\hline $\begin{array}{l}\text { Rosa subcanina (Christ) Dalla } \\
\text { Torre et Sarnth. }\end{array}$ & Rosaceae & $P$ & CEU & & 32 & \\
\hline Rubus caesius L. & Rosaceae & $\mathrm{P}$ & EUAS & & $\begin{array}{c}3,4,5,14,15,16,22,37,42,44,55,65 \\
70\end{array}$ & V\&al-2019 \\
\hline Rubus candicans Weihe ex Rchb. & Rosaceae & $\mathrm{P}$ & EUAS & & 32 & \\
\hline Rubus idaeus L. & Rosaceae & $\mathrm{P}$ & WISP & & $3,10,32,34,35,38,39,40$ & \\
\hline $\begin{array}{l}\text { Rubus ulmifolius Schott. (incl. } R \text {. } \\
\text { heteromorphus Ripart ex Genev) }\end{array}$ & Rosaceae & $\mathrm{P}$ & ME-atl & & $\begin{array}{c}1,4,5,13,14,15,18,21,22,23,24,25 \\
26,27,29,30,31,37,43,44,47,48,50 \\
51,57,59,61,62,64 ?\end{array}$ & $\begin{array}{l}\text { VRZ-2012, as R. } \\
\text { heteromorphus Ripart ex } \\
\text { Genev; V\&al-2019 }\end{array}$ \\
\hline Rumex acetosella $\mathrm{L}$. & Polygonaceae & $\mathrm{H}$ & WISP & & $11,32,44$ & \\
\hline Rumex acetosa L. ssp. acetosa & Polygonaceae & $\mathrm{H}$ & WISP & & 21 & $\begin{array}{l}\text { V\&al-2019 as R. acetosa } \\
\text { L. }\end{array}$ \\
\hline Rumex conglomeratus Murray & Polygonaceae & $\mathrm{H}$ & WISP & & $3,4,14,16,22,23,28$ & \\
\hline Rumex crispus L. & Polygonaceae & $\mathrm{H}$ & WISP & & $1,4,5,16,23,25,27,43,46$ & V\&al-2019 \\
\hline Rumex pulcher L. & Polygonaceae & $\mathrm{H}$ & SEU-po & & $3,5,16,20,22,23,25,27,37,43,50$ & V\&al-2019 \\
\hline $\begin{array}{l}\text { Romulea bulbocodium (L.) Sebast. } \\
\text { et Mauri }\end{array}$ & Iridaceae & G & ME-circ & & 2 & \\
\hline Ruscus aculeatus L. & Asparagaceae & G & ME-po & LC & $4,14,16,22,37,42,69,70$ & V\&al-2019 \\
\hline Ruscus hypoglossum L. & Asparagaceae & G & SEU-po & NT & Significant Landscape of Sutina & V\&al-2020 \\
\hline Salix appendiculata Vill. & Salicaceae & $\mathrm{P}$ & CEU & & $32,34,36$ & \\
\hline Salix alba L. & Salicaceae & $\mathrm{P}$ & EUAS & & $46,69,70$ & \\
\hline Salix caprea $\mathrm{L}$. & Salicaceae & $\mathrm{P}$ & EUAS & & $32,36,41$ & \\
\hline Salix cinerea $\mathrm{L}$. & Salicaceae & $\mathrm{P}$ & EUAS & & $32,34,35,37,40,64$ & \\
\hline Salix purpurea L. & Salicaceae & $\mathrm{P}$ & EUAS & & $22,28,46,47,59,64,69,70$ & V\&al-2019 \\
\hline Salvia glutinosa L. & Lamiaceae & $\mathrm{H}$ & EUAS & & 14 & Vi-1847:187 \\
\hline Salvia officinalis L. & Lamiaceae & $\mathrm{Ch}$ & ME-eu & & $7(4861547,5607414)$ & V\&al-2019 \\
\hline $\begin{array}{l}\text { Salvia pratensis L. (incl. Salvia } \\
\text { bertolonii Vis.) } \\
\end{array}$ & Lamiaceae & $\mathrm{H}$ & EURO & & $\begin{array}{c}1,4,5,7,15,16,17,20,21,25,26,42 \\
43,45,46,48,49,51,52,59,60,65\end{array}$ & V\&al-2019 \\
\hline Salvia sclarea $\mathrm{L}$. & Lamiaceae & $\mathrm{H}$ & SEU-me & & $3,21,22,23,25,46,66$ & \\
\hline
\end{tabular}




\begin{tabular}{|c|c|c|c|c|c|c|}
\hline Taxon & Family & $\begin{array}{l}\text { Life } \\
\text { form }\end{array}$ & Geoelement & Status & Localities (finding sites) & $\begin{array}{l}\text { Previously recorded } \\
\text { findings }\end{array}$ \\
\hline Salvia verbenaca $\mathrm{L}$. & Lamiaceae & $\mathrm{H}$ & ME-atl & & 14 & V\&al-2019 \\
\hline Salvia verticillata $\mathrm{L}$. & Lamiaceae & $\mathrm{H}$ & EURO & & $15,50,58,65,66,67$ & \\
\hline Sambucus ebulus L. & Caprifoliaceae & G & EURO & & $\begin{array}{c}3,4,5,13,14,16,21,25,27,30,32,35 \\
37,66,70\end{array}$ & V\&al-2019 \\
\hline Sambucus nigra L. & Caprifoliaceae & $P$ & EURO & & $\begin{array}{c}1,13,14,15,16,20,21,24,27,32,34 \\
35,39,40,43,50,58,59,63,69\end{array}$ & Pe-1832:112; V\&al-2019 \\
\hline Sambucus racemosa L. & Caprifoliaceae & $\mathrm{P}$ & $\mathrm{CIHO}$ & & 32 & \\
\hline $\begin{array}{l}\text { Sanguisorba minor Scop. ssp. } \\
\text { minor }\end{array}$ & Rosaceae & $\mathrm{H}$ & EUAS & & $\begin{array}{c}4,8,9,11,27,40,44,47,50,53,57,63 \\
64,65,66,69\end{array}$ & \\
\hline $\begin{array}{l}\text { Sanguisorba minor Scop. ssp. } \\
\text { muricata Briq. }\end{array}$ & Rosaceae & $\mathrm{H}$ & SEU-me & & $\begin{array}{c}1,2,5,6,7,10,11,12,13,14,15,16 \\
17,18,19,20,21,23,25,26,28,30,31 \\
32,34,35,36,37,38,39,40,41,42,43 \\
45,46,48,49,51,52,53,54,55,56,58 \\
59,60,62,63,69,70\end{array}$ & $\begin{array}{l}\text { Tr-1992 as S. muricata } \\
\text { (Spach) Gremli; } \\
\text { V\&al-2019 }\end{array}$ \\
\hline Sanicula europaea L. & Apiaceae & $\mathrm{H}$ & WISP & & 63 & V\&al-2019 \\
\hline Saponaria officinalis L. & Caryophyllaceae & $\mathrm{H}$ & WISP & & $1,3,4,13,22,23,66,70$ & \\
\hline Satureja cuneifolia Ten. & Lamiaceae & $\mathrm{Ch}$ & ME-ilape & & $6,23,25,26,46$ & \\
\hline $\begin{array}{l}\text { Satureja montana L. ssp. variegata } \\
\text { (Host) P. W. Ball }\end{array}$ & Lamiaceae & $\mathrm{Ch}$ & ME-po & & $\begin{array}{c}1,2,6,8,10,18,22,26,27,31,37,40 \\
43,45,48,49,50,51,53,54,56,59,65 \\
66,69\end{array}$ & $\begin{array}{l}\text { Je-1952, Tr-1992, } \\
\text { VRZ-2012, Se\&al-2016, } \\
\text { M\&K-2016 and }\end{array}$ \\
\hline Satureja montana L. ssp. montana & Lamiaceae & $\mathrm{Ch}$ & ME-po & & 29,32 & $\begin{array}{l}\text { V\&al-2019, as S. montana } \\
\text { L. }\end{array}$ \\
\hline $\begin{array}{l}\text { Satureja subspicata Vis. ssp. } \\
\text { subspicata }\end{array}$ & Lamiaceae & $\mathrm{Ch}$ & ME-ilade & $\mathrm{LC}$ & $\begin{array}{c}6,8,9,11,18,26,40,51,53,54,56,59 \\
65,66,69\end{array}$ & $\begin{array}{l}\text { Se\&al-2016 and } \\
\text { V\&al-2019 as S. } \\
\text { subspicata Vis.; }\end{array}$ \\
\hline Saxifraga bulbifera L. & Saxifragaceae & $\mathrm{H}$ & SEEU & & 13,37 & V\&al-2019 \\
\hline $\begin{array}{l}\text { Saxifraga marginata Sternb. (incl. } \\
\text { S. coriophylla Griseb.) }\end{array}$ & Saxifragaceae & $\mathrm{Ch}$ & ILBA-end & & Svilaja mountain range & $\begin{array}{l}\text { L\&R-1989, as } S . \\
\text { coriophylla Griseb. }\end{array}$ \\
\hline $\begin{array}{l}\text { Saxifraga rotundifolia L. ssp. } \\
\text { rotundifolia }\end{array}$ & Saxifragaceae & $\mathrm{H}$ & SEU-mo & LC & $11,32,35,36,38$ & $\begin{array}{l}\text { V\&al-2019 as } S \text {. } \\
\text { rotundifolia L. }\end{array}$ \\
\hline Saxifraga tridactylites L. & Saxifragaceae & $\mathrm{T}$ & WISP & & $\begin{array}{c}6,10,11,12,20,30,32,34,36,37,40 \\
52,66\end{array}$ & V\&al-2019 \\
\hline $\begin{array}{l}\text { Scabiosa columbaria L. ssp. } \\
\text { columbaria }\end{array}$ & Dipsacaceae & $\mathrm{H}$ & EUAS & LC & 55 & $\begin{array}{l}\text { V\&al-2019 as } C \text {. } \\
\text { columbaria } \mathrm{L} \text {. }\end{array}$ \\
\hline Scabiosa cinerea Lapeyr. ex Lam. & Dipsacaceae & $\mathrm{H}$ & ME-ilade & & 69 & $\begin{array}{l}\text { LRS-1989 as S. } \\
\text { leucophylla Borbás }\end{array}$ \\
\hline Scabiosa delminiana Abadžić & Dipsacaceae & $\mathrm{H}$ & ME-ilade & end & $40,41,51$ & \\
\hline Scabiosa triandra $\mathrm{L}$. & Dipsacaceae & $\mathrm{H}$ & SEU-me & & $4,7,9,13,15,19,32,41,46,59,66,69 ?$ & V\&al-2019 \\
\hline $\begin{array}{l}\text { Scandix pecten-veneris L. ssp. } \\
\text { pecten-veneris }\end{array}$ & Apiaceae & $\mathrm{T}$ & WISP & NT & 30 & \\
\hline Scilla autumnalis L. & Asparagaceae & G & ME-po & & $6,15,18,26,27,31,48,49,50,51,59$ & V\&al-2019 \\
\hline Scirpus holoschoenus L. & Cyperaceae & G & ME-circ & NT & $1,22,23,25,28,46,47,61$ & V\&al-2019 \\
\hline Scirpus lacustris L. ssp. lacustris & Cyperaceae & Hy & WISP & & 22 & $\begin{array}{l}\text { V\&al-2019 as S. lacustris } \\
\text { L. }\end{array}$ \\
\hline $\begin{array}{l}\text { Scirpus maritimus L. ssp. } \\
\text { maritimus }\end{array}$ & Cyperaceae & Hy & WISP & NT & $22,23,28$ & \\
\hline Scleranthus annuus L. & Caryophyllaceae & $\mathrm{T}$ & WISP & & $11,29,44$ & \\
\hline Sclerochloa dura (L.) P. Beauv. & Poaceae & $\mathrm{T}$ & SEU-me & & 22 & $\begin{array}{l}\text { Vi-1842:82 as Poa dura } \\
\text { Scop. }\end{array}$ \\
\hline Scolymus hispanicus L. & Cichoriaceae & $\mathrm{H}$ & ME-circ & & $3,5,13,21,22,23,25,68$ & \\
\hline $\begin{array}{l}\text { Scorzonera austriaca Willd. ssp. } \\
\text { austriaca }\end{array}$ & Cichoriaceae & $\mathrm{H}$ & SEU-po & & 41 & \\
\hline $\begin{array}{l}\text { Scorzonera austriaca Willd. ssp. } \\
\text { bupleurifolia (Pouzolz) Bonnier }\end{array}$ & Cichoriaceae & $\mathrm{H}$ & SEU-po & $\begin{array}{l}\text { end, } \\
\text { sp }\end{array}$ & 12 & \\
\hline Scorzonera laciniata L. & Cichoriaceae & $\mathrm{H}$ & ILBA-end & & $5,20,23,28$ & \\
\hline Scorzonera villosa Scop. & Cichoriaceae & $\mathrm{H}$ & ME-ilseu & & $\begin{array}{c}1,5,6,17,23,25,26,29,30,31,35,39 \\
43,45,46,48,51,52,53,55,56,57,58 \\
62\end{array}$ & $\begin{array}{l}\text { Tr-1992; Se\&al-2016; } \\
\text { V\&al-2019 }\end{array}$ \\
\hline Scrophularia bosniaca Beck & Scrophulariaceae & $\mathrm{H}$ & ME-ilade & $\begin{array}{l}\text { end, } \\
\mathrm{DD}, \\
\mathrm{sp}\end{array}$ & 10,32 & \\
\hline
\end{tabular}




\begin{tabular}{|c|c|c|c|c|c|c|}
\hline Taxon & Family & \begin{tabular}{|c|} 
Life \\
form
\end{tabular} & Geoelement & Status & Localities (finding sites) & $\begin{array}{l}\text { Previously recorded } \\
\text { findings }\end{array}$ \\
\hline $\begin{array}{l}\text { Scrophularia canina L. ssp. bicolor } \\
\text { (Sibth. et Sm.) Greuter }\end{array}$ & Scrophulariaceae & $\mathrm{H}$ & SEU-me & & $\begin{array}{c}1,4,6,7,8,13,15,17,21,22,23,25 \\
27,29,30,31,32,36,37,38,40,42,45 \\
46,47,50,51,53,55,58,59,61,65,69\end{array}$ & V\&al-2019 as S. canina L. \\
\hline $\begin{array}{l}\text { Scrophularia heterophylla Willd. } \\
\text { ssp. laciniata (Waldst. et Kit.) } \\
\text { Maire et Petitm }\end{array}$ & Scrophulariaceae & $\mathrm{H}$ & ILBA-end & & 34,35 & Ja-1908 as S. laciniata \\
\hline Scrophularia nodosa L. & Scrophulariaceae & $\mathrm{H}$ & $\mathrm{CIHO}$ & & Significant Landscape of Sutina & V\&al-2019 \\
\hline Sedum acre L. & Crassulaceae & $\mathrm{Ch}$ & WISP & & $\begin{array}{c}1,7,9,11,17,20,21,23,25,27,30,31 \\
37,38,42,43,45,46,52,55,58,62\end{array}$ & VRZ-2012; V\&al-2019 \\
\hline Sedum album $\mathrm{L}$. & Crassulaceae & $\mathrm{Ch}$ & EUAS & & $14,27,35,65$ & \\
\hline Sedum atratum $\mathrm{L}$. & Crassulaceae & $\mathrm{T}$ & SEU-mo & & 33,34 & Ja-1908 \\
\hline Sedum dasyphyllum $\mathrm{L}$. & Crassulaceae & $\mathrm{Ch}$ & SEU-me & & 14 & M\&K-2016 \\
\hline Sedum hispanicum L. & Crassulaceae & $\mathrm{T}$ & SEU-po & & $14,21,30,31,32,35,40,43,46,55$ & VRZ-2012; V\&al-2019 \\
\hline Sedum ochroleucum Chaix & Crassulaceae & $\mathrm{Ch}$ & SEU-me & & $\begin{array}{c}8,9,10,11,17,21,23,31,32,34,36,38 \\
39,40,52,53,55,58,61,65,67\end{array}$ & VRZ-2012; V\&al-2019 \\
\hline Sedum rupestre L. & Crassulaceae & $\mathrm{Ch}$ & CEU & & 1,32 & \\
\hline Sedum sexangulare $\mathrm{L}$. & Crassulaceae & $\mathrm{Ch}$ & SEU-me & & $\begin{array}{c}1,4,5,6,7,8,9,11,15,17,18,19,20 \\
21,22,23,25,26,27,30,32,34,35,36, \\
37,39,40,42,43,44,46,47,49,50,53 \\
55,58,59,60,65,66\end{array}$ & Tr-1992 \\
\hline $\begin{array}{l}\text { Sedum telephium L. ssp. maximum } \\
\text { (L.) Krock }\end{array}$ & Crassulaceae & $\mathrm{H}$ & EURO & & 20,27 & \\
\hline Selinum carvifolia $(\mathrm{L}.) \mathrm{L}$. & Apiaceae & $\mathrm{H}$ & EUAS & & 41 & \\
\hline Sempervivum marmoreum Griseb. & Crassulaceae & $\mathrm{Ch}$ & ILBA-end & $\mathrm{sp}$ & $10,11,12$ & LRS-1989; V\&al-2019 \\
\hline Sempervivum tectorum $\mathrm{L}$. & Crassulaceae & $\mathrm{Ch}$ & CUAD & $\mathrm{sp}$ & $1,23,27^{*}, 37^{*}, 42^{*}$ & \\
\hline Senecio angulatus L. f. & Asteraceae & $\mathrm{Ch}$ & CUAD & & 1 & \\
\hline Senecio aquaticus Hill & Asteraceae & $\mathrm{H}$ & CEU & & 22 & \\
\hline $\begin{array}{l}\text { Senecio doronicum (L.) L. ssp. } \\
\text { doronicum }\end{array}$ & Asteraceae & $\mathrm{H}$ & SEU-mo & & 33,34 & \\
\hline Senecio vulgaris L. & Asteraceae & $\mathrm{T}$ & WISP & & $\begin{array}{c}3,6,13,14,16,24,27,28,37,49,50 \\
68,69\end{array}$ & V\&al-2019 \\
\hline Senecio squalidus L. & Asteraceae & $\mathrm{H}$ & SEEU & & 32 & \\
\hline Serapias parviflora Parl. & Orchidaceae & G & ME-circ & $\mathrm{sp}$ & Muć & Kr-2005:380 \\
\hline Serapias vomeracea (Burm.) Briq. & Orchidaceae & G & ME-atl & $\begin{array}{l}\mathrm{VU}, \\
\mathrm{sp}\end{array}$ & Muć Gornji & Kr-2005:382 \\
\hline $\begin{array}{l}\text { Serratula radiata (Waldst. et Kit.) } \\
\text { M.Bieb. ssp. cetingensis (Rohlena) } \\
\text { Hayek }\end{array}$ & Asteraceae & $\mathrm{H}$ & EEUPO & $\begin{array}{l}\text { end, } \\
\text { sp }\end{array}$ & 41 & $\begin{array}{l}\text { V\&al-2019 as S. radiata } \\
\text { (Waldst. et Kit.) M.Bieb. }\end{array}$ \\
\hline Serratula tinctoria $\mathrm{L}$. & Asteraceae & $\mathrm{H}$ & EUAS & & $\begin{array}{c}\text { "in sylvaticis et pratis umbrosis inter } \\
\text { Dernis et Verlika" }\end{array}$ & Vi-1847:52 \\
\hline $\begin{array}{l}\text { Seseli elatum L. ssp. gouanii } \\
\text { (Koch) P. W. Ball }\end{array}$ & Apiaceae & $\mathrm{H}$ & ILBA-end & $\begin{array}{c}\text { end, } \\
\text { sp }\end{array}$ & 34,41 & \\
\hline $\begin{array}{l}\text { Seseli montanum L. ssp. } \\
\text { tommassinii (Rchb. F.) Arcang. }\end{array}$ & Apiaceae & $\mathrm{H}$ & ME-ilseu & $\begin{array}{l}\text { end, } \\
\text { sp }\end{array}$ & $\begin{array}{c}8,9,15,18,19,26,27,30,31,48,49 \\
50,51,54,55,56,58,59,65,66\end{array}$ & V\&al-2019 \\
\hline Seseli pallasii Besser & Apiaceae & $\mathrm{H}$ & SEU-po & & $2,24,25,41$ & V\&al-2019 \\
\hline Seseli tomentosum Vis. & Apiaceae & $\mathrm{H}$ & ME-ilade & $\begin{array}{c}\text { end, } \\
\text { NT, sp }\end{array}$ & 26 & V\&al-2019 \\
\hline Seseli tortuosum L. & Apiaceae & $\mathrm{H}$ & SEU-me & & $1,4,5$ & \\
\hline $\begin{array}{l}\text { Sesleria autumnalis (Scop.) F.W. } \\
\text { Schultz }\end{array}$ & Poaceae & $\mathrm{H}$ & ME-ilseu & & \begin{tabular}{|c}
$2,6,10,11,18,19,26,30,31,32,36$ \\
$37,47,51,52,57,59,60,61,64,65,69$
\end{tabular} & $\begin{array}{l}\text { Je-1952; Se\&al-2016; } \\
\text { V\&al-2019 }\end{array}$ \\
\hline $\begin{array}{l}\text { Sesleria robusta Schott, Nyman et } \\
\text { Kotschy }\end{array}$ & Poaceae & $\mathrm{H}$ & SEU-me & & $10,33,34$ & LRS-1989 \\
\hline $\begin{array}{l}\text { Sesleria tenuifolia Schrad. ssp. } \\
\text { tenuifolia }\end{array}$ & Poaceae & $\mathrm{H}$ & ILBA-end & & $\begin{array}{c}8,9,10,11,12,31,33,34,35,40,41 \\
52,53,56,57,64,69\end{array}$ & $\begin{array}{l}\text { L\&R-1989 as S. juncifolia } \\
\text { s. lat.; LRS-1989 as S. } \\
\text { tenuifolia Schrad. ssp. } \\
\text { interrupta Vis.; } \\
\text { V\&al-2019 as. S. } \\
\text { tenuifolia Schrad. }\end{array}$ \\
\hline Setaria gussonei Kerguélen & Poaceae & $\mathrm{T}$ & WISP & & 1,14 & \\
\hline Setaria italica (L.) P.Beauv. & Poaceae & $\mathrm{T}$ & WISP & & $4,14,22$ & \\
\hline Setaria pumila (Poir.) Schult. & Poaceae & $\mathrm{T}$ & WISP & & 14,22 & \\
\hline Setaria verticillata (L.) P.Beauv. & Poaceae & $\mathrm{T}$ & WISP & & $3,4,14,24,68$ & \\
\hline
\end{tabular}




\begin{tabular}{|c|c|c|c|c|c|c|}
\hline Taxon & Family & \begin{tabular}{|c|} 
Life \\
form
\end{tabular} & Geoelement & Status & Localities (finding sites) & $\begin{array}{l}\text { Previously recorded } \\
\text { findings }\end{array}$ \\
\hline Setaria viridis (L.) P.Beauv. & Poaceae & $\mathrm{T}$ & EUAS & & $4,5,13,22,27,37,49,50,66,68$ & \\
\hline Sherardia arvensis L. & Rubiaceae & $\mathrm{T}$ & WISP & & $\begin{array}{c}1,2,16,17,20,26,27,37,42,43,45 \\
60,61,69,70\end{array}$ & V\&al-2019 \\
\hline Sideritis montana L. ssp. montana & Lamiaceae & $\mathrm{T}$ & ME-po & & 21,31 & \\
\hline Silene conica L. & Caryophyllaceae & $\mathrm{T}$ & EUAS & & 14 & Pe-1832:120 \\
\hline Silene italica (L.) Pers ssp. italica & Caryophyllaceae & $\mathrm{H}$ & SEU-me & & $19,25,35,37,42,45,53,55,66,69$ & \\
\hline $\begin{array}{l}\text { Silene italica (L.) Pers. ssp. } \\
\text { nemoralis (Waldst. et Kit.) Nyman }\end{array}$ & Caryophyllaceae & $\mathrm{H}$ & SEU-me & & Significant Landscape of Sutina & V\&al-2019 \\
\hline $\begin{array}{l}\text { Silene latifolia Poir. ssp. alba } \\
\text { (Mill.) Greuter et Bourdet }\end{array}$ & Caryophyllaceae & $\mathrm{H}$ & EUAS & & $\begin{array}{c}1,4,5,11,13,14,26,27,32 ?, 36,40 \\
43,48,49,50,55,58,58,65,66,70\end{array}$ & $\begin{array}{l}\text { Ma-1992 as S. alba (Mill.) } \\
\text { E.H.L. Krause; } \\
\text { V\&al-2019 }\end{array}$ \\
\hline Silene latifolia Poir. ssp. latifolia & Caryophyllaceae & $\mathrm{H}$ & EUAS & & $\begin{array}{c}1,3,13,14,15,16,17,20,21,23,24 \\
25,29,30,31,32,37,42,44,45,46,69\end{array}$ & \\
\hline Silene multicaulis Guss. & Caryophyllaceae & $\mathrm{H}$ & ME-ilape & & Svilaja mountain range & LRS-1989 \\
\hline Silene otites (L.) Wibel & Caryophyllaceae & $\mathrm{H}$ & SEU-po & & $1,6,18,43,55,57,58,59$ & V\&al-2019 \\
\hline Silene paradoxa L. & Caryophyllaceae & $\mathrm{H}$ & SEU-atl & & 42,69 & \\
\hline Silene saxifraga $\mathrm{L}$. & Caryophyllaceae & $\mathrm{H}$ & SEU-mo & & $33,34,41$ & Ja-1908 \\
\hline Silene sendtneri Boiss. & Caryophyllaceae & $\mathrm{H}$ & ILBA-end & $\begin{array}{c}\text { end, } \\
\text { sp }\end{array}$ & 41 & LRS-1989 \\
\hline Silene viridiflora $\mathrm{L}$. & Caryophyllaceae & $\mathrm{H}$ & SEU-po & & 41 & \\
\hline $\begin{array}{l}\text { Silene vulgaris (Moench) Garcke } \\
\text { ssp. angustifolia Hayek }\end{array}$ & Caryophyllaceae & $\mathrm{H}$ & SEU-me & & $\begin{array}{c}1,2,4,6,7,13,17,23,25,27,29,31 \\
32,36,37,38,40,42,43,45,48,53,55 \\
58,63,65,66\end{array}$ & VRZ-2012; V\&al-2019 \\
\hline Sinapis arvensis L. & Brassicaceae & $\mathrm{T}$ & WISP & & $1,3,4,21,24,28$ & \\
\hline Sisymbrium irio L. & Brassicaceae & $\mathrm{T}$ & EUAS & & "in devexitatibus montis Svilaja" & Vi-1872:123 \\
\hline Sisymbrium officinale (L.) Scop. & Brassicaceae & $\mathrm{T}$ & WISP & & $\begin{array}{c}1,5,16,21,23,25,27,30,44,45,47 \\
50,66,68,69\end{array}$ & Ma-1992; V\&al-2019 \\
\hline $\begin{array}{l}\text { Sixalix atropurpurea (Forssk.) } \\
\text { Greuter et Burdet ssp. maritima } \\
\text { (L.) Greuter et Burdet }\end{array}$ & Dipsacaceae & $\mathrm{H}$ & ME-circ & & 27 & \\
\hline Smyrnium olusatrum L. & Apiaceae & $\mathrm{H}$ & ME-atl & & $2,21,66$ & \\
\hline Smyrnium perfoliatum $\mathrm{L}$. & Apiaceae & $\mathrm{H}$ & ME-circ & & $\begin{array}{c}4,13,14,15,16,18,21,27,29,30,42 \\
46,47,65\end{array}$ & \begin{tabular}{|l|} 
Pe-1832:122 as $S$. \\
dioscoridis Spr.; \\
Vi-1852:68; V\&al-2019
\end{tabular} \\
\hline Solanum dulcamara L. & Solanaceae & $\mathrm{P}$ & WISP & & 28 & \\
\hline Solanum lycopersicum L. & Solanaceae & $\mathrm{T}$ & CUAD & & $\begin{array}{c}3^{*}, 4^{*}, 14,16^{*}, 22^{*}, 24,25^{*}, 26,27,37^{*}, \\
43^{*}, 49^{*}, 66^{*}\end{array}$ & \\
\hline Solanum nigrum L. & Solanaceae & $\mathrm{T}$ & WISP & & $4,14,25^{*}, 27$ & V\&al-2019 \\
\hline Solanum tuberosum L. & Solanaceae & $\mathrm{T}$ & CUAD & & $\begin{array}{c}3^{*}, 4,13^{*}, 14^{*}, 16^{*}, 27,28^{*}, 30^{*}, 37^{*}, \\
43^{*}, 45,47^{*}, 69\end{array}$ & \\
\hline $\begin{array}{l}\text { Solanum villosum Mill. ssp. alatum } \\
\text { (Moench) Dostál }\end{array}$ & Solanaceae & $\mathrm{T}$ & EUAS & & 27,50 & \\
\hline Solidago gigantea Aiton & Asteraceae & G & CUAD & inv & $3 * 4$ & \\
\hline $\begin{array}{l}\text { Solidago virgaurea L. ssp. } \\
\text { virgaurea }\end{array}$ & Asteraceae & $\mathrm{H}$ & EUAS & & 51,64 & V\&al-2019 \\
\hline Sonchus arvensis $\mathrm{L}$. & Cichoriaceae & $\mathrm{H}$ & WISP & & 21,28 & \\
\hline Sonchus asper (L.) Hill ssp. asper & Cichoriaceae & $\mathrm{T}$ & ME-circ & & 47 & \\
\hline $\begin{array}{l}\text { Sonchus asper (L.) Hill ssp. } \\
\text { glaucescens (Jord.) Ball }\end{array}$ & Cichoriaceae & $\mathrm{H}$ & ME-circ & & $3,16,21,29,45$ & \\
\hline Sonchus oleraceus L. & Cichoriaceae & $\mathrm{T}$ & WISP & & $13,14,23,24,27,49$ & V\&al-2019 \\
\hline Sonchus tenerrimus L. & Cichoriaceae & $\mathrm{T}$ & ME-circ & & 14,47 & M\&K-2016 \\
\hline Sorbus aria (L.) Crantz & Rosaceae & $P$ & WISP & & $\begin{array}{c}9,11,31,32,34,35,36,38,39,40,41 \\
47,48,49,50,51,52,55,56,58,61,65 \\
66\end{array}$ & V\&al-2019 \\
\hline Sorbus aucuparia L. ssp. aucuparia & Rosaceae & $\mathrm{P}$ & EUAS & & 32 & $\begin{array}{l}\text { V\&al-2019 as } S . \\
\text { aucuparia } \mathrm{L} .\end{array}$ \\
\hline Sorbus domestica L. & Rosaceae & $\mathrm{P}$ & CUAD & & $4,13,14,19,42,59,65,70$ & \\
\hline Sorbus torminalis (L.) Crantz & Rosaceae & $\mathrm{P}$ & EUAS & & 32,63 & $\begin{array}{l}\text { Vi-1852:246 as Pyrus } \\
\text { torminalis Ehrh.; Je-1952; } \\
\text { V\&al-2019 }\end{array}$ \\
\hline Sorghum halepense (L.) Pers. & Poaceae & G & WISP & inv & 4,14 & \\
\hline
\end{tabular}




\begin{tabular}{|c|c|c|c|c|c|c|}
\hline Taxon & Family & \begin{tabular}{|c|} 
Life \\
form
\end{tabular} & Geoelement & Status & Localities (finding sites) & $\begin{array}{c}\text { Previously recorded } \\
\text { findings }\end{array}$ \\
\hline Spartium junceum L. & Fabaceae & $\mathrm{P}$ & ME-circ & & $47,57,61$ & V\&al-2019 \\
\hline Spiraea cana Waldst. et Kit. & Rosaceae & $\mathrm{Ch}$ & ME-ilade & $\mathrm{sp}$ & Significant Landscape of Sutina & V\&al-2019 \\
\hline Spiraea media Schmidt & Rosaceae & $\mathrm{P}$ & EEUPO & & $32,35,36,38,39,40,65$ & $\begin{array}{l}\text { V-1952:256 as S. } \\
\text { chamaedryfolia L.; Ja-1908 }\end{array}$ \\
\hline Spiranthes spiralis (L.) Chevall. & Orchidaceae & G & EURO & $\mathrm{sp}$ & Muć & Kr-2005:384 \\
\hline Stachys annua (L.) L. & Lamiaceae & $\mathrm{T}$ & EURO & & $13,14,68$ & \\
\hline $\begin{array}{l}\text { Stachys cretica L. ssp. salviifolia } \\
\text { (Ten.) Rech. f. }\end{array}$ & Lamiaceae & $\mathrm{H}$ & ME-ilape & & 8,16 & V\&al-2019 \\
\hline Stachys recta L. & Lamiaceae & $\mathrm{H}$ & SEU-po & & $11,33,48$ & \\
\hline Stachys subcrenata Vis. & Lamiaceae & $\mathrm{H}$ & ME-ilape & & $9,32,34,36,38$ & V\&al-2019 \\
\hline Stachys sylvatica L. & Lamiaceae & $\mathrm{H}$ & EUAS & & $14,35,58$ & Vi-1847:206; V\&al-2019 \\
\hline Stachys thirkei K.Koch & Lamiaceae & $\mathrm{H}$ & ME-east & & $\begin{array}{c}1,3,4,5,16,17,19,21,22,23,25,26 \\
43,45,46,58,61,62,70\end{array}$ & \\
\hline Stellaria media (L.) Vill. & Caryophyllaceae & $\mathrm{T}$ & WISP & & $2,14,16,37,49,50,61$ & V\&al-2019 \\
\hline Stellaria pallida (Dumort) Piré & Caryophyllaceae & $\mathrm{T}$ & EUAS & & $2,20,44,60$ & \\
\hline $\begin{array}{l}\text { Stipa pennata L. ssp. eriocaulis } \\
\text { (Borbás) Martinovský et Skalický }\end{array}$ & Poaceae & $\mathrm{H}$ & SEU-me & & $\begin{array}{c}1,2,8,9,11,13,17,21,23,25,31,32, \\
41,48,50,51,52,53,54,56,58,59,65 \\
67\end{array}$ & $\begin{array}{l}\text { V\&al-2019 as S. pennata } \\
\text { L. }\end{array}$ \\
\hline Stipa pulcherrima K. Koch & Poaceae & $\mathrm{H}$ & SEU-po & & Svilaja mountain range & LRS-1989 \\
\hline Symphytum tuberosum L. & Boraginaceae & G & SEU-co & & 11,32 & Vi-1847:255; V\&al-2019 \\
\hline Syringa vulgaris L. & Oleaceae & $P$ & CUAD & & $\begin{array}{c}1,3^{*}, 13^{*}, 14,24,27,28^{*}, 30,37,43^{*} \\
45^{*}, 49^{*}, 66,70\end{array}$ & \\
\hline Tagetes patula $\mathrm{L}$. & Asteraceae & $\mathrm{T}$ & CUAD & & $1,14,27,49^{*}, 50$ & \\
\hline Tamus communis $\mathrm{L}$. & Dioscoreaceae & G & SEU-me & & $\begin{array}{c}2,3,5,14,16,20,22,25,28,30,37,42 \\
44,45,46,60,63,69,70\end{array}$ & V\&al-2019 \\
\hline $\begin{array}{l}\text { Tanacetum corymbosum (L.) Sch. } \\
\text { Bip }\end{array}$ & Asteraceae & $\mathrm{H}$ & CEU & & 32 & $\begin{array}{l}\text { Vi-1847:88 as } \\
\text { Chrysanthemum } \\
\text { corymbosum L. }\end{array}$ \\
\hline $\begin{array}{l}\text { Tanacetum parthenium (L.) Sch. } \\
\text { Bip. }\end{array}$ & Asteraceae & $\mathrm{H}$ & WISP & & $1,3,13,27,42$ & \\
\hline Tanacetum vulgare $\mathrm{L}$. & Asteraceae & $\mathrm{H}$ & CUAD & & $23^{*}, 46^{*}, 59$ & \\
\hline Taraxacum hoppeanum Griseb. & Cichoriaceae & $\mathrm{H}$ & SEU-mo & & 36 & \\
\hline Taraxacum decipiens Raunk. & Cichoriaceae & $\mathrm{H}$ & SEU-me & & 2,44 & \\
\hline Taraxacum laevigatum auct. croat. & Cichoriaceae & $\mathrm{H}$ & SEU-me & & $6,10,29,30,44,47,52,53,57,61,66$ & \\
\hline Taraxacum officinale Webber & Cichoriaceae & $\mathrm{H}$ & WISP & & $\begin{array}{c}1,4,10,11,13,14,15,16,20,27,28 \\
29,32,37,42,47,49,51,60,63,64,66 \\
69,70\end{array}$ & $\begin{array}{l}\text { L\&R-1989 as T. officinale } \\
\text { Wigg. (s. lat.); } \\
\text { V\&al-2019 }\end{array}$ \\
\hline $\begin{array}{l}\text { Taraxacum palustre (Lyons) } \\
\text { Symons }\end{array}$ & Cichoriaceae & $\mathrm{H}$ & EUAS & & 60 & \\
\hline Teucrium arduini $\mathrm{L}$ & Lamiaceae & $\mathrm{H}$ & ME-ilade & $\begin{array}{l}\text { end, } \\
\text { DD, } \\
\text { sp }\end{array}$ & $38,39,40,41,51,52$ & \\
\hline Teucrium chamaedrys L. & Lamiaceae & $\mathrm{Ch}$ & SEU-po & & \begin{tabular}{|c|}
$1,2,3,4,6,7,8,10,11,13,15,16,17$ \\
$18,20,21,23,25,26,27,28,29,30,31$ \\
$34,35,36,37,38,39,40,41,42,43,44$ \\
$45,46,47,48,49,50,51,52,53,54,55$ \\
$56,57,58,60,61,62,64,65,66,69,70$ \\
\end{tabular} & V\&al-2019 \\
\hline Teucrium montanum $\mathrm{L}$. & Lamiaceae & $\mathrm{Ch}$ & SEU-me & & $\begin{array}{c}1,2,6,7,8,9,10,11,23,25,26,27,31 \\
32,34,35,36,38,39,40,41,47,48,50 \\
51,52,53,54,56,57,58,59,61,62,64 \\
65,69\end{array}$ & $\begin{array}{l}\text { Je-1952; Tr-1992; } \\
\text { V\&al-2019 }\end{array}$ \\
\hline $\begin{array}{l}\text { Teucrium polium L. ssp. capitatum } \\
\text { (L.) Arcang. }\end{array}$ & Lamiaceae & $\mathrm{Ch}$ & ME-po & & $\begin{array}{c}1,2,7,8,15,16,17,18,19,21,25,26 \\
27,31,37,42,43,44,45,46,47,48,49 \\
50,54,61,69,70\end{array}$ & $\begin{array}{l}\text { Tr-1992 as T. polium L.; } \\
\text { V\&al-2019 }\end{array}$ \\
\hline Teucrium scordioides Schreb. & Lamiaceae & $\mathrm{H}$ & ME-atl & & 46 & \\
\hline Thalictrum aquilegifolium $\mathrm{L}$ & Ranunculaceae & $\mathrm{H}$ & EUAS & & $27,37,39,40,41,53$ & \\
\hline Thalictrum minus $\mathrm{L}$. & Ranunculaceae & $\mathrm{H}$ & WISP & & $\begin{array}{c}2,9,11,13,15,16,20,26,33,34,35 \\
36,41,51,64,65,66\end{array}$ & Vi-1852:78; V\&al-2019 \\
\hline $\begin{array}{l}\text { Thesium divaricatum Jan. ex Mert. } \\
\text { et Koch }\end{array}$ & Santalaceae & $\mathrm{H}$ & ME-circ & & $8,9,23,24,26,31,43,45,49,50,51,59$ & $\begin{array}{l}\text { Tr-1992; VRZ-2012; } \\
\text { V\&al-2019 }\end{array}$ \\
\hline Thesium linophyllon L. & Santalaceae & $\mathrm{H}$ & SEU-po & & $19,25,33,39,65,70 ?$ & Vi-1842:222 \\
\hline Thlaspi arvense L. & Brassicaceae & $\mathrm{T}$ & WISP & & Significant Landscape of Sutina & V\&al-2019 \\
\hline
\end{tabular}




\begin{tabular}{|c|c|c|c|c|c|c|}
\hline Taxon & Family & \begin{tabular}{|c|} 
Life \\
form
\end{tabular} & Geoelement & Status & Localities (finding sites) & $\begin{array}{l}\text { Previously recorded } \\
\text { findings }\end{array}$ \\
\hline Thlaspi perfoliatum L. & Brassicaceae & $\mathrm{T}$ & EUAS & & $2,29,30,36,66,70$ & \\
\hline Thlaspi praecox Wulfen & Brassicaceae & $\mathrm{H}$ & ME-ilseu & & $\begin{array}{c}8,9,10,11,12,33,34,39,41,51,53 \\
57,58,61,66,67\end{array}$ & V\&al-2019 \\
\hline Thymus bracteosus Vis. ex Benth. & Lamiaceae & $\mathrm{Ch}$ & ME-ilade & $\begin{array}{l}\text { end, } \\
\text { sp }\end{array}$ & $\begin{array}{c}26,32,38,39,40,41,46,51,53,56,58 \\
59\end{array}$ & Vi-1847:193; V\&al-2019 \\
\hline Thymus longicaulis C.Presl & Lamiaceae & $\mathrm{Ch}$ & ME-ilape & & $\begin{array}{c}1,2,3,8,10,11,12,13,16,17,19,20 \\
26,29,30,31,32,36,37,40,41,42,44 \\
45,47,55,57,59,60,61,64,65,66,67, \\
69,70\end{array}$ & $\begin{array}{l}\text { Je-1952; VRZ-2012; } \\
\text { V\&al-2019 }\end{array}$ \\
\hline Thymus striatus Vahl & Lamiaceae & $\mathrm{Ch}$ & ILBA-bap & & 33,34 & \\
\hline Tilia platyphyllos Scop. & Tiliaceae & $\mathrm{P}$ & EURO & & $1,22^{*}, 43^{*}$ & \\
\hline Tordylium apulum L. & Apiaceae & $\mathrm{T}$ & ME-circ & & $1,2,3,21,29,30,43,45$ & V\&al-2019 \\
\hline Tordylium maximum $\mathrm{L}$. & Apiaceae & $\mathrm{T}$ & EUAS & & $1,3,16,22,23,28,46$ & Ma-1992 \\
\hline $\begin{array}{l}\text { Torilis arvensis (Huds.) Link ssp. } \\
\text { arvensis }\end{array}$ & Apiaceae & $\mathrm{T}$ & EURO & & $23,25,27$ & \\
\hline $\begin{array}{l}\text { Torilis arvensis (Huds.) Link ssp. } \\
\text { purpurea (Ten.) Hayek }\end{array}$ & Apiaceae & $\mathrm{T}$ & ME-circ & & $1,3,5$ & \\
\hline Torilis nodosa (L.) Gaertn. & Apiaceae & $\mathrm{T}$ & ME-atl & & $21,23,25,27,45$ & \\
\hline Tragopogon balcanicus Velen. & Cichoriaceae & $\mathrm{H}$ & ILBA-end & & $24,25,32,38,55$ & \\
\hline Tragopogon dubius Scop. & Cichoriaceae & $\mathrm{H}$ & SEU-po & & 14,21 & V\&al-2019 \\
\hline $\begin{array}{l}\text { Tragopogon pratensis L. ssp. } \\
\text { orientalis (L.) Čelak. }\end{array}$ & Cichoriaceae & $\mathrm{H}$ & EUAS & & Significant Landscape of Sutina & V\&al-2019 \\
\hline Tragopogon tommasinii Sch.Bip. & Cichoriaceae & $\mathrm{H}$ & ME-ilade & & $\begin{array}{c}8,9,11,16,17,28,32,37,41,42,47 \\
49,51,53,55,58,59,62,63,65,70\end{array}$ & \\
\hline Tragus racemosus (L.) All. & Poaceae & $\mathrm{T}$ & SEU-me & & 27 & \\
\hline Tribulus terrestris L. & Zygophyllaceae & $\mathrm{T}$ & SEU-me & & 4,27 & \\
\hline Trifolium alpestre $\mathrm{L}$. & Fabaceae & $\mathrm{H}$ & SEU-mo & & $\begin{array}{c}11,19,24,25,32,33,34,35,36,40,43 \\
55,65,67\end{array}$ & V\&al-2019 \\
\hline Trifolium angustifolium L. & Fabaceae & $\mathrm{T}$ & ME-circ & & $5,22,23,46,47$ & V\&al-2019 \\
\hline Trifolium arvense L. & Fabaceae & $\mathrm{T}$ & EUAS & & $8,11,55$ & V\&al-2019 \\
\hline Trifolium campestre Schreber & Fabaceae & $\mathrm{T}$ & WISP & & $\begin{array}{c}5,11,14,16,17,20,21,23,27,28,31 \\
35,37,42,43,45,46,50,51,55,58,63 \\
69,70\end{array}$ & $\begin{array}{l}\text { Ma-1964; Tr-1992; } \\
\text { V\&al-2019 }\end{array}$ \\
\hline Trifolium dalmaticum Vis. & Fabaceae & $\mathrm{T}$ & ME-ilseu & $\begin{array}{l}\text { end, } \\
\text { sp }\end{array}$ & $\begin{array}{c}1,8,11,14,16,21,23,24,25,27,43 \\
48,49,58,59\end{array}$ & \\
\hline Trifolium diffusum Erhardt & Fabaceae & $\mathrm{T}$ & SEU-po & & 49 & \\
\hline $\begin{array}{l}\text { Trifolium fragiferum } \text { L. ssp. } \\
\text { bonannii (C. Presl) Soják }\end{array}$ & Fabaceae & $\mathrm{H}$ & SEU-me & & 22 & $\begin{array}{l}\text { V\&al-2019 as T. } \\
\text { incarnatum L. }\end{array}$ \\
\hline $\begin{array}{l}\text { Trifolium incarnatum L. ssp. } \\
\text { incarnatum }\end{array}$ & Fabaceae & $\mathrm{T}$ & ME-atl & & 59 & \\
\hline $\begin{array}{l}\text { Trifolium incarnatum L. ssp. } \\
\text { molinerii (Balb.ex Hornem.) Syme }\end{array}$ & Fabaceae & $\mathrm{T}$ & ME-atl & DD & $\begin{array}{c}2,5,8,11,16,17,20,21,27,29,37,43 \\
44,45,48,50,55,58,65,67\end{array}$ & \\
\hline Trifolium montanum L. & Fabaceae & $\mathrm{H}$ & EUAS & & $32,36,55$ & V\&al-2019 \\
\hline Trifolium nigrescens Viv. & Fabaceae & $\mathrm{T}$ & ME-circ & & $2,5,27$ & \\
\hline Trifolium ocroleucon Huds. & Fabaceae & $\mathrm{H}$ & SEU-po & & $9,24,32,65$ & \\
\hline Trifolium pannonicum Jacq. & Fabaceae & $\mathrm{H}$ & CEU & $\begin{array}{l}\mathrm{VU}, \\
\mathrm{sp}\end{array}$ & $8,25,55$ & \\
\hline Trifolium patens Schreb. & Fabaceae & $\mathrm{T}$ & SEU-co & & $14,16,28,32,65$ & \\
\hline Trifolium pratense $\mathrm{L}$. & Fabaceae & $\mathrm{H}$ & EUAS & & $\begin{array}{c}3,4,5,8,11,13,14,15,16,17,18,20 \\
23,26,27,28,29,32,37,40,42,43,45 \\
46,47,48,49,50,54,55,59,63,65,66 \\
69,70\end{array}$ & V\&al-2019 \\
\hline $\begin{array}{l}\text { Trifolium repens L. ssp. prostratum } \\
\text { Nyman }\end{array}$ & Fabaceae & $\mathrm{H}$ & ME-circ & & $5,7,13,17,19,21,27,42,44,66$ & \\
\hline Trifolium repens L. ssp. repens & Fabaceae & $\mathrm{H}$ & WISP & & $\begin{array}{c}11,13,14,15,23,26,27,28,42,45,48 \\
50,55,70\end{array}$ & $\begin{array}{l}\text { Ma-1964, V\&al-2019 as } \\
\text { T. repens L. }\end{array}$ \\
\hline Trifolium resupinatum $\mathrm{L}$. & Fabaceae & $\mathrm{T}$ & ME-po & $\begin{array}{l}\mathrm{VU}, \\
\mathrm{sp}\end{array}$ & $1,5,23,28$ & \\
\hline Trifolium rubens $\mathrm{L}$. & Fabaceae & $\mathrm{H}$ & SEU-me & & 17 & V\&al-2019 \\
\hline
\end{tabular}




\begin{tabular}{|c|c|c|c|c|c|c|}
\hline Taxon & Family & \begin{tabular}{|c|} 
Life \\
form
\end{tabular} & Geoelement & Status & Localities (finding sites) & $\begin{array}{c}\text { Previously recorded } \\
\text { findings }\end{array}$ \\
\hline Trifolium scabrum L. & Fabaceae & $\mathrm{T}$ & ME-circ & & $1,8,23$ & \\
\hline Trifolium squamosum L. & Fabaceae & $\mathrm{T}$ & ME-circ & & 28 & \\
\hline Trifolium stellatum L. & Fabaceae & $\mathrm{T}$ & ME-circ & & 26,50 & \\
\hline $\begin{array}{l}\text { Trifolium striatum L. ssp. } \\
\text { tenuiflorum (Ten.) Arcang. }\end{array}$ & Fabaceae & $\mathrm{T}$ & EUAS & & 16,43 & \\
\hline Trifolium subterraneum L. & Fabaceae & $\mathrm{T}$ & ME-atl & & 37 & \\
\hline Trigonella esculenta Willd. & Fabaceae & $\mathrm{T}$ & ME-eu & & $14,17,23$ & \\
\hline Trinia glauca (L.) Dumort. & Apiaceae & $\mathrm{H}$ & ILBA-end & & 33,34 & $\begin{array}{l}\text { Vi-1852:29 as T. vulgaris } \\
\text { DC. }\end{array}$ \\
\hline Trisetum flavescens (L.) P.Beauv. & Poaceae & $\mathrm{H}$ & $\mathrm{CIHO}$ & & $1,13,15,16,28,66$ & \\
\hline $\begin{array}{l}\text { Tulipa sylvestris L. (incl. T. } \\
\text { grisebachiana Pant.) }\end{array}$ & Liliaceae & G & SEU-me & NT & $10,32,36$ & $\begin{array}{l}\text { Vi-1842:133, A-1911:62 } \\
\text { as T. grisebachiana; } \\
\text { M\&al-2013; V\&al-2019 }\end{array}$ \\
\hline Tussilago farfara $\mathrm{L}$. & Asteraceae & G & EUAS & & $\begin{array}{c}10,32,36,38,44,47,50,55,57,58,62 \\
68\end{array}$ & V\&al-2019 \\
\hline Typha angustifolia $\mathrm{L}$. & Typhaceae & G & WISP & & Significant Landscape of Sutina & V\&al-2019 \\
\hline Typha domingensis (Pers.) Steud. & Typhaceae & G & SEU-me & & $3,22,28,44,46,47,59,64$ & \\
\hline Ulmus glabra Huds. & Ulmaceae & $\mathrm{P}$ & WISP & & Significant Landscape of Sutina & V\&al-2019 \\
\hline Ulmus minor Mill. & Ulmaceae & $P$ & WISP & & $\begin{array}{c}2,4,5,11,13,14,16,21,22,23,29,37 \\
42,44,45,46,47,51,59,60,66\end{array}$ & V\&al-2019 \\
\hline $\begin{array}{l}\text { Urospermum picroides (L.) Scop. } \\
\text { ex F. W. Schmidt }\end{array}$ & Cichoriaceae & $\mathrm{T}$ & ME-circ & & 37 & \\
\hline Urtica dioica $\mathrm{L}$. & Urticaceae & $\mathrm{H}$ & WISP & & \begin{tabular}{|c|}
$1,3,4,5,10,11,12,13,14,16,20,21$ \\
$23,24,27,29,30,32,35,37,42,45,48$ \\
$49,50,54,58,59,61,65,66,69,70$
\end{tabular} & Ma-1992; V\&al-2019 \\
\hline Urtica urens L. & Urticaceae & $\mathrm{T}$ & WISP & & $6,14,46,50$ & Ma-1964 \\
\hline $\begin{array}{l}\text { Vaccaria hispanica (Miller) } \\
\text { Rauschert }\end{array}$ & Caryophyllaceae & $\mathrm{T}$ & WISP & $\mathrm{CR}, \mathrm{sp}$ & 3 & \\
\hline Valeriana officinalis L. & Valerianaceae & $\mathrm{H}$ & EUAS & & $10,20,32$ & \\
\hline Valeriana tuberosa L. & Valerianaceae & $\mathrm{H}$ & SEU-me & & $\begin{array}{c}2,6,11,12,29,30,41,47,53,57,61 \\
66,67\end{array}$ & V\&al-2019 \\
\hline Valerianella dentata (L.) Pollich & Valerianaceae & $\mathrm{T}$ & SEU-me & & 23,28 & V\&al-2019 \\
\hline Valerianella locusta (L.) Laterrade & Valerianaceae & $\mathrm{T}$ & ME-circ & & 42 & \\
\hline Veratrum nigrum L. & Melanthiaceae & G & EUAS & & 32,65 & Vi-1842:155 \\
\hline Verbascum pulverulentum Vill. & Scrophulariaceae & $\mathrm{H}$ & SEU-atl & & $3,4,13,27,59,66$ & \\
\hline Verbascum blattaria $\mathrm{L}$. & Scrophulariaceae & $\mathrm{H}$ & SEU-po & & $23,25,50$ & \\
\hline $\begin{array}{l}\text { Verbascum chaixii Vill. ssp. } \\
\text { austriacum (Schott ex Roem. et } \\
\text { Schult.) Hayek }\end{array}$ & Scrophulariaceae & $\mathrm{H}$ & CEU & DD & $7,10,11,21,32,33,40,41,53,58$ & $\begin{array}{l}\text { Vi- } 1847: 157 \text { as } V . \text { nigrum } \\
\text { § orientale; V\&al-2019 }\end{array}$ \\
\hline Verbascum densiflorum Bertol. & Scrophulariaceae & $\mathrm{H}$ & EURO & & 56,59 & $\begin{array}{l}\text { Pe-1832:138 as V. thapsus } \\
\text { L. }\end{array}$ \\
\hline Verbascum lychnitis L. & Scrophulariaceae & $\mathrm{H}$ & EUAS & & $1,5,16,43$ & \\
\hline Verbascum macrurum Ten. & Scrophulariaceae & $\mathrm{H}$ & ILBA-bap & & 41 & \\
\hline Verbascum nigrum L. & Scrophulariaceae & $\mathrm{H}$ & EURO & & 14 & Pe-1832:138 \\
\hline Verbascum phlomoides L. & Scrophulariaceae & $\mathrm{H}$ & EURO & & Significant Landscape of Sutina & V\&al-2019 \\
\hline Verbascum phoeniceum L. & Scrophulariaceae & $\mathrm{T}$ & SEU-po & & $2,6,17,48,51,67$ & V\&al-2019 \\
\hline Verbascum pulverulentum Vill. & Scrophulariaceae & $\mathrm{H}$ & SEU-me & & 21,25 & V\&al-2019 \\
\hline Verbascum sinuatum L. & Scrophulariaceae & $\mathrm{H}$ & ME-circ & & 7 & \\
\hline Verbena officinalis L. & Verbenaceae & $\mathrm{H}$ & WISP & & $\begin{array}{c}1,3,4,5,6,13,14,15,16,20,22,23 \\
27,28,35,42,63,65,68\end{array}$ & V\&al-2019 \\
\hline $\begin{array}{l}\text { Veronica anagallis-aquatica L. ssp. } \\
\text { anagallis-aquatica }\end{array}$ & Scrophulariaceae & $\mathrm{H}$ & EURO & LC & $14,22,23$ & $\begin{array}{l}\text { Vi-1847:171 as } V . \\
\text { anagallis L.; V\&al-2019 } \\
\text { as V. anagallis-aquatica L. }\end{array}$ \\
\hline Veronica arvensis L. & Scrophulariaceae & $\mathrm{T}$ & EUAS & & $11,14,16,17,20,29,37,40,69$ & V\&al-2019 \\
\hline Veronica austriaca L. ssp. austriaca & Scrophulariaceae & $\mathrm{H}$ & SEU-mo & & $17,39,49$ & $\begin{array}{l}\text { De-2010 as V. austriaca } \\
\text { ssp. dentata (F. W. } \\
\text { Schmidt) Watzl }\end{array}$ \\
\hline $\begin{array}{l}\text { Veronica austriaca L. ssp. jacquinii } \\
\text { (Baumg.) Eb.Fisch. }\end{array}$ & Scrophulariaceae & $\mathrm{H}$ & EEUPO & & $2,9,11,26,30,32,37,41,42,49,51,52$ & $\begin{array}{l}\text { Je-1952 as V. austriaca } \\
\text { ssp. orbiculata var. } \\
\text { hercegovinica; V\&al-2019 }\end{array}$ \\
\hline Veronica chamaedrys L. & Scrophulariaceae & $\mathrm{H}$ & EUAS & & $11,13,36,37,42,47,60,61,65,69$ & \\
\hline
\end{tabular}




\begin{tabular}{|c|c|c|c|c|c|c|}
\hline Taxon & Family & $\begin{array}{c}\text { Life } \\
\text { form }\end{array}$ & Geoelement & Status & Localities (finding sites) & $\begin{array}{l}\text { Previously recorded } \\
\text { findings }\end{array}$ \\
\hline Veronica cymbalaria Bodard & Scrophulariaceae & $\mathrm{T}$ & EUAS & & 29,37 & \\
\hline Veronica hederifolia L. & Scrophulariaceae & $\mathrm{T}$ & EUAS & & 37,60 & \\
\hline Veronica officinalis L. & Scrophulariaceae & $\mathrm{H}$ & $\mathrm{CIHO}$ & & $11,21,35,63$ & Vi-1847:169; V\&al-2019 \\
\hline Veronica persica Poir. & Scrophulariaceae & $\mathrm{T}$ & WISP & inv & $\begin{array}{c}13,14,16,20,24,27,28,29,30,37,47 \\
60,66,68,69,70\end{array}$ & $\begin{array}{l}\text { Ma-1964 as V. } \\
\text { tournefortii C.C. Gmel.; } \\
\text { V\&al-2019 }\end{array}$ \\
\hline Veronica polita Fr. & Scrophulariaceae & $\mathrm{T}$ & EUAS & & 2 & V\&al-2019 \\
\hline Veronica serpyllifolia L. & Scrophulariaceae & $\mathrm{H}$ & WISP & & 11 & \\
\hline Veronica triloba Opiz & Scrophulariaceae & $\mathrm{T}$ & ME-east & & $29,60,69$ & \\
\hline Viburnum lantana L. & Scrophulariaceae & $\mathrm{P}$ & SEU-me & & 32,63 & Je-1952; V\&al-2019 \\
\hline Viburnum opulus L. & Caprifoliaceae & $\mathrm{P}$ & EUAS & & $32,64,65,66,69,70$ & \\
\hline $\begin{array}{l}\text { Vicia angustifolia L. ssp. } \\
\text { angustifolia }\end{array}$ & Fabaceae & $\mathrm{T}$ & EURO & & $\begin{array}{c}1,2,11,14,16,17,20,21,25,27,29 \\
30,37,42,43,44,45,46,47,48,51,55 \\
58,60,61,65,69,70\end{array}$ & \\
\hline Vicia bithynica (L.) L. & Fabaceae & $\mathrm{T}$ & ME-circ & & Significant Landscape of Sutina & V\&al-2019 \\
\hline Vicia cracca L. & Fabaceae & $\mathrm{H}$ & EUAS & & $11,32,44$ & V\&al-2019 \\
\hline Vicia grandiflora Scop. & Fabaceae & $\mathrm{T}$ & EEUPO & & $2,14,16,37,42,46,47$ & V\&al-2019 \\
\hline Vicia hybrida L. & Fabaceae & $\mathrm{T}$ & ME-circ & & 37,69 & \\
\hline Vicia lutea L. & Fabaceae & $\mathrm{T}$ & ME-circ & & 37 & \\
\hline Vicia narbonensis L. & Fabaceae & $\mathrm{T}$ & ME-circ & & 70 & \\
\hline $\begin{array}{l}\text { Vicia ochroleuca Ten. ssp. dinara } \\
\text { (K. Malý) Rohlena }\end{array}$ & Fabaceae & $\mathrm{H}$ & ME-ilade & $\begin{array}{l}\text { end, } \\
\text { sp }\end{array}$ & $21,31,32,35,40,53$ & VRZ-2012; V\&al-2019 \\
\hline Vicia oroboides Wulfen & Fabaceae & $\mathrm{H}$ & ILBA-end & & 36 & \\
\hline $\begin{array}{l}\text { Vicia pannonica Crantz ssp. striata } \\
\text { (M. Bieb.) Nyman }\end{array}$ & Fabaceae & $\mathrm{T}$ & SEU-me & & 32,45 & \\
\hline $\begin{array}{l}\text { Vicia sativa L. ssp. cordata } \\
\text { (Hoppe) Batt. }\end{array}$ & Fabaceae & $\mathrm{T}$ & SEU-po & & 45 & V\&al-2019 as V. sativa L. \\
\hline $\begin{array}{l}\text { Vicia tenuissima (M.Bieb.) Schinz } \\
\text { et Thell. }\end{array}$ & Fabaceae & $\mathrm{T}$ & ME-circ & & 9,11 & \\
\hline Vicia villosa Roth & Fabaceae & $\mathrm{T}$ & EEUPO & & $\begin{array}{c}1,4,5,8,14,17,20,23,28,43,45,46 \\
55\end{array}$ & V\&al-2019 \\
\hline Vinca major $\mathrm{L}$. & Apocynaceae & $\mathrm{Ch}$ & CUAD & & $1,14,27,65,66^{*}$ & \\
\hline $\begin{array}{l}\text { Vincetoxicum fuscatum (Hornem.) } \\
\text { Reichenb. }\end{array}$ & Asclepiadaceae & $\mathrm{H}$ & SEEU & & $41,51,52$ & \\
\hline $\begin{array}{l}\text { Vincetoxicum hirundinaria Medik. } \\
\text { ssp. adriaticum (Beck) Markgr. }\end{array}$ & Asclepiadaceae & $\mathrm{H}$ & ME-ilade & $\begin{array}{l}\text { end, } \\
\text { LC, sp }\end{array}$ & $\begin{array}{c}13,19,26,27,31,33,34,40,41,48 \\
52 ?, 54,55,56,59,62\end{array}$ & V\&al-2019 \\
\hline Viola arvensis Murray & Violaceae & $\mathrm{T}$ & WISP & & $\begin{array}{c}2,14,16,17,20,24,25,27,29,37,42 \\
44,47,48,65\end{array}$ & V\&al-2019 \\
\hline Viola hirta L. & Violaceae & $\mathrm{H}$ & EUAS & & Significant Landscape of Sutina & V\&al-2020 \\
\hline Viola kitaibeliana Schultes & Violaceae & $\mathrm{T}$ & ME-circ & & 2,42 & \\
\hline Viola odorata L. & Violaceae & $\mathrm{H}$ & WISP & & & V\&al-2019 \\
\hline $\begin{array}{l}\text { Viola reichenbachiana Jord. Ex } \\
\text { Boreau }\end{array}$ & Violaceae & $\mathrm{H}$ & EUAS & & 10,64 & V\&al-2019 \\
\hline $\begin{array}{l}\text { Viola suavis M.Bieb. ssp. adriatica } \\
\text { (Freyn) Haesler }\end{array}$ & Violaceae & $\mathrm{H}$ & ME-ilade & $\begin{array}{c}\text { end, } \\
\text { sp }\end{array}$ & $10,36,53,57,66,67$ & V\&al-2019 \\
\hline $\begin{array}{l}\text { Viola tricolor L. ssp. subalpina } \\
\text { Gaudin }\end{array}$ & Violaceae & $\mathrm{T}$ & EURO & & $10,11,12,26$ & \\
\hline Vitis vinifera L. ssp. vinifera & Vitaceae & $\mathrm{P}$ & CUAD & & $\begin{array}{c}1,4,13^{*}, 15,16,20^{*}, 22,24,25,27,37^{*}, \\
42^{*}, 43^{*}, 45,49^{*}, 69^{*}, 70\end{array}$ & \\
\hline $\begin{array}{l}\text { Vitis vinifera L. ssp. sylvestris (C. } \\
\text { C. Gmel.) Hegi }\end{array}$ & Vitaceae & $\mathrm{P}$ & EUAS & & $5,43,70$ & \\
\hline Vulpia bromoides (L.) Gray & Poaceae & $\mathrm{T}$ & EUAS & & 9,35 & \\
\hline Vulpia ciliata Dumort. & Poaceae & $\mathrm{T}$ & SEU-me & & $1,23,43,45,55,66$ & \\
\hline Vulpia fasciculata (Forssk.) Samp. & Poaceae & $\mathrm{T}$ & ME-atl & NT & 20 & \\
\hline Vulpia myuros (L.) C.C.Gmel. & Poaceae & $\mathrm{T}$ & WISP & & $5,14,21,25,58$ & \\
\hline Xanthium spinosum $\mathrm{L}$. & Asteraceae & $\mathrm{T}$ & WISP & inv & 25 & \\
\hline $\begin{array}{l}\text { Xanthium strumarium L. ssp. } \\
\text { italicum (Moretti) D. Löve }\end{array}$ & Asteraceae & $\mathrm{T}$ & WISP & inv & $1,22,23,28,43$ & \\
\hline Xeranthemum inapertum (L.) Mill. & Asteraceae & $\mathrm{T}$ & SEU-po & & $1,43,69^{*}$ & \\
\hline Zea mays L. & Poaceae & $\mathrm{T}$ & CUAD & & $1^{*}, 3,4^{*}, 14,16^{*}, 21,22^{*}, 23$ & \\
\hline
\end{tabular}




\section{APPENDIX 2}

Tab. 2. The plant taxa found only in cultivation on Mt Svilaja (abbreviations are explained in the section Materials and Methods).

\begin{tabular}{|c|c|c|c|}
\hline Taxon & Family & Life form & Finding sites (Localities) \\
\hline Actinidia chinensis Planch. & Actinidiaceae & $\mathrm{P}$ & $70^{*}$ \\
\hline Albizia julibrissin Durazz. & Fabaceae & $\mathrm{P}$ & $5^{*}$ \\
\hline Allium sativum $\mathrm{L}$. & Amaryllidaceae & $\mathrm{G}$ & $14^{*}, 25^{*}, 30^{*}, 37^{*}, 43^{*}$ \\
\hline Balsamita major Desf. & Asteraceae & $\mathrm{Ch}$ & $1^{*}, 23^{*}$ \\
\hline Buddleja davidii Franch & Buddlejaceae & $\mathrm{P}$ & $25^{*}$ \\
\hline Buxus sempervirens L. & Buxaceae & $\mathrm{P}$ & $70^{*}$ \\
\hline Canna indica L. & Cannaceae & $\mathrm{G}$ & $1^{*}$ \\
\hline Capsicum annuum L. & Solanaceae & $\mathrm{T}$ & $3^{*}, 16^{*}, 22^{*}, 24^{*}$ \\
\hline Cedrus atlantica (Endl.) Carriere & Pinaceae & $\mathrm{P}$ & $14^{*}$ \\
\hline Cicer arietinum $\mathrm{L}$. & Fabaceae & $\mathrm{T}$ & $16^{*}, 25^{*}, 28^{*}, 37^{*}$ \\
\hline Commelina communis $\mathrm{L}$. & Commelinaceae & $\mathrm{G}$ & $27^{*}$ \\
\hline $\begin{array}{l}\text { Cortaderia selloana (Schult. \& Schult.f.) Asch. \& } \\
\text { Graebn. }\end{array}$ & Poaceae & $\mathrm{H}$ & $1^{*}$ \\
\hline Cucumis sativus L. & Cucurbitaceae & $\mathrm{T}$ & $1^{*}, 16^{*}, 24^{*}, 70^{*}$ \\
\hline Cydonia oblonga Mill. & Rosaceae & $\mathrm{P}$ & $24^{*}, 37^{*}, 70^{*}$ \\
\hline Cylindropuntia imbricata (Haw.) F. M. Knuth & Cactaceae & $\mathrm{P}$ & $22^{*}$ \\
\hline Dahlia variabilis (Willd.) Desf. & Asteraceae & G & $23^{*}, 24^{*}$ \\
\hline Daucus carota L. ssp. satious (Hoffm.) Arcang. & Apiaceae & $\mathrm{T}$ & $16^{*}, 22^{*}, 37^{*}, 70^{*}$ \\
\hline Delosperma cooperi (Hook.f.) L.Bolus* & Aizoaceae & $\mathrm{Ch}$ & $27^{*}$ \\
\hline Deutzia scabra Thunb. & Hydrangeaceae & $\mathrm{P}$ & $14^{*}$ \\
\hline Erysimum cheiri (L.) Crantz & Brassicaceae & $\mathrm{Ch}$ & $27^{*}$ \\
\hline Euonymus japonica L.f. & Celastraceae & $\mathrm{P}$ & $27^{*}, 43^{*}$ \\
\hline $\begin{array}{l}\text { Euphorbia characias L. ssp. wulfenii (Hoppe ex Koch) } \\
\text { A. M. Sm. }\end{array}$ & Euphorbiaceae & $\mathrm{P}$ & $43^{*}$ \\
\hline Fragaria ananassa Duchesne* & Rosaceae & $\mathrm{H}$ & $70^{*}$ \\
\hline Hibiscus syriacus L. & Malvaceae & $\mathrm{P}$ & $4^{*}, 16^{*}, 27^{*}, 66^{*}$ \\
\hline Hydrangea macrophylla (Thunb.) Ser. & Hydrangeaceae & $\mathrm{P}$ & $27^{*}, 69^{*}$ \\
\hline Iberis sempervirens L. & Brassicaceae & $\mathrm{Ch}$ & $70^{*}$ \\
\hline Impatiens balfourii Hooker $\mathrm{f}$. & Balsaminaceae & $\mathrm{T}$ & $27^{*}$ \\
\hline Lagenaria vulgaris Ser. & Cucurbitaceae & $\mathrm{T}$ & $16^{*}$ \\
\hline Lavandula $\mathrm{x}$ intermedia Emeric ex Loisel. & Lamiaceae & $\mathrm{P}$ & $1,37^{*}, 43^{*}, 70^{*}$ \\
\hline Limonium sinuatum (L.) Miller* & Plumbaginaceae & $\mathrm{H}$ & $65^{*}$ \\
\hline Lobularia maritima (L.) Desv. & Brassicaceae & $\mathrm{H}$ & $37^{*}$ \\
\hline Petroselinum crispum (Mill.) A. W. Hill & Apiaceae & $\mathrm{H}$ & $27^{*}, 31^{*}, 37^{*}, 49^{*}, 49,50^{*}, 70^{*}$ \\
\hline Petunia hybrida Vilm. & Solanaceae & $\mathrm{T}$ & $46^{*}$ \\
\hline Phaseolus vulgaris L. & Fabaceae & $\mathrm{T}$ & $14^{*}, 16^{*}, 25,27,37^{*}, 50^{*}$ \\
\hline Philadelphus coronarius L. & Hydrangeaceae & $\mathrm{P}$ & $66^{*}$ \\
\hline Photinia $x$ fraseri Dress* & Rosaceae & $\mathrm{P}$ & $16^{*}$ \\
\hline Pisum sativum L. ssp. sativum & Fabaceae & $\mathrm{T}$ & $16^{*}, 28^{*}, 37^{*}, 47^{*}, 70^{*}$ \\
\hline Poinciana gilliesii Hook. & Fabacae & $\mathrm{P}$ & $23^{*}$ \\
\hline Portulaca grandiflora Hooker & Portulacaceae & $\mathrm{T}$ & $27^{*}$ \\
\hline Prunus armeniaca $\mathrm{L}$. & Rosaceae & $\mathrm{P}$ & $27^{*}, 37^{*}$ \\
\hline Prunus laurocerasus L. & Rosaceae & $\mathrm{P}$ & $1^{*}, 14^{*}, 16^{*}, 70^{*}$ \\
\hline Rhus typhina L. & Anacardiaceae & $\mathrm{P}$ & $14^{*}, 70^{*}$ \\
\hline Ribes rubrum L. & Grossulariaceae & $\mathrm{P}$ & $32 *, 70^{*}$ \\
\hline Rosmarinus officinalis L. & Lamiaceae & $\mathrm{P}$ & $\begin{array}{c}1^{*}, 4^{*}, 16^{*}, 23^{*}, 27^{*}, 37^{*}, 43^{*} \\
47^{*}, 47^{*}, 66^{*}\end{array}$ \\
\hline Salix babylonica L. & Salicaceae & $\mathrm{P}$ & $28^{*}, 69^{*}$ \\
\hline Sedum palmeri S.Watson* & Crassulaceae & $\mathrm{Ch}$ & $27^{*}$ \\
\hline Sorghum bicolor (L.) Moench & Poaceae & $\mathrm{T}$ & $14^{*}, 22^{*}$ \\
\hline Symphoricarpos albus (L.) S. F. Blake & Caprifoliaceae & $\mathrm{P}$ & $13^{*}$ \\
\hline Thuja orientalis L. & Cupressaceae & $\mathrm{P}$ & $65^{*}$ \\
\hline Trachycarpus fortunei (Hook.) H.Wendl.* & Arecaceae & $\mathrm{P}$ & $1^{*}$ \\
\hline Triticum aestivum $\mathrm{L}$. & Poaceae & $\mathrm{T}$ & $3^{*}, 69^{*}$ \\
\hline Tropaeolum majus L. & Tropaeolaceae & $\mathrm{T}$ & $65^{*}$ \\
\hline Tulipa gesneriana L.* & Liliaceae & $\mathrm{G}$ & $30^{*}$ \\
\hline Weigela florida (Bigelow) A. DC. & Caprifoliaceae & $\mathrm{P}$ & $70^{*}$ \\
\hline Wisteria sinensis (Sims) Sweet & Fabaceae & $\mathrm{P}$ & $14^{*}$ \\
\hline Yucca gloriosa $\mathrm{L}$ & Agavaceae & $\mathrm{P}$ & $22^{*}, 46^{*}$ \\
\hline
\end{tabular}

